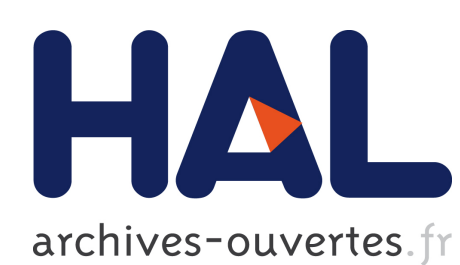

archives-ouvertes

\title{
Measurement of the photon electroproduction cross section at JLAB with the goal of performing a Rosenbluth separation of the DVCS contribution
}

Alejandro Miguel Martí Jiménez-Argüello

\section{- To cite this version:}

Alejandro Miguel Martí Jiménez-Argüello. Measurement of the photon electroproduction cross section at JLAB with the goal of performing a Rosenbluth separation of the DVCS contribution. Other. Université Paris Sud - Paris XI; Universidad de Valencia (Espagne), 2014. English. $<$ NNT : 2014PA112135>. <tel-01061951>

\section{HAL Id: tel-01061951 \\ https://tel.archives-ouvertes.fr/tel-01061951}

Submitted on 8 Sep 2014

HAL is a multi-disciplinary open access archive for the deposit and dissemination of scientific research documents, whether they are published or not. The documents may come from teaching and research institutions in France or abroad, or from public or private research centers.
L'archive ouverte pluridisciplinaire HAL, est destinée au dépôt et à la diffusion de documents scientifiques de niveau recherche, publiés ou non, émanant des établissements d'enseignement et de recherche français ou étrangers, des laboratoires publics ou privés. 


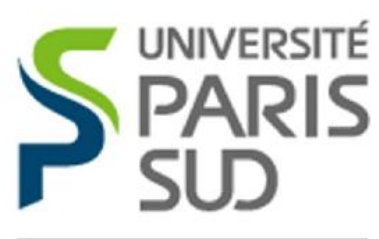

Comprendre le monde, construire l'avenir ${ }^{8}$

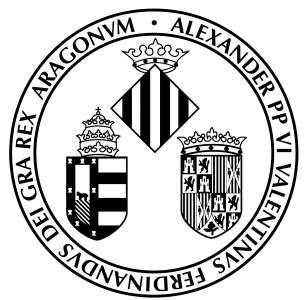

VNIVERSITAT E Ö̈VALĖNCIA

\section{UNIVERSITÉ PARIS-SUD \\ ÉCOLE DOCTORALE: Particules, Noyaux et Cosmos. \\ Institut de Physique Nucléaire d'Orsay. \\ DISCIPLINE: Physique des particules.}

\section{THÈSE DE DOCTORAT}

soutenue le 11/07/2014

par

Alejandro Miguel Martí Jiménez-Argüello.

\section{Mesure de la section efficace de l'électroproduction de photons à JLab dans le but d'effectuer une Séparation Rosenbluth de la contribution DVCS.}

Directeur de thèse: Carlos Muñoz Camacho. Chargé de recherche au C.N.R.S.

Paris-Sud Université.

Co-directeur de thèse: Santiago Noguera Puchol. Professeur de Physique Théorique.

Universitat de València.

Composition du jury:

Président du jury: Michel Guidal. Directeur de recherches au C.N.R.S. Paris-Sud Université. Rapporteur: Julie Roche. Professeur associé. Ohio University, USA.

Rapporteur: Vicente Vento Torres. Professeur de Physique Théorique. Universitat de València. Examinateur: Helene Fonvieille. Directrice de recherches au C.N.R.S. Université Blaise Pascal. Examinateur: Juan Miguel Nieves Pamplona. Chercheur. Institut de Física Corpuscular (CSIC). Examinateur: José Díaz Medina. Professeur de Physique Nucléaire. Universitat de València. 





\section{Contents}

$\begin{array}{ll}\text { Introduction } & 7\end{array}$

1 Deeply Virtual Compton Scattering 11

1.1 Elastic scattering: form factors . . . . . . . . . . . . . . . . 12

1.2 Deeply Inelastic scattering: parton distributions . . . . . . . . . . . . . . . 14

1.3 Exclusive reactions: DVCS . . . . . . . . . . . . . . . 18

1.4 Deeply photon electroproduction cross section . . . . . . . . . . . . 22

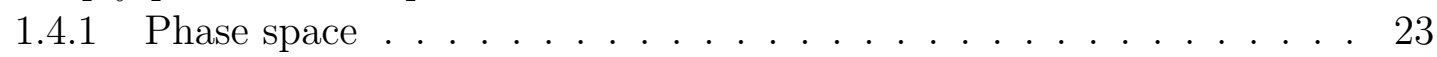

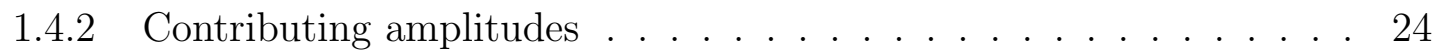

1.5 Experiment goals . . . . . . . . . . . . . . . . . . . . . 31

2 Experimental setup $\quad 36$

2.1 Jefferson Lab . . . . . . . . . . . . . . . . . . . . . . . . 36

2.2 Hall A . . . . . . . . . . . . . . . . . . . . . . . . 37

2.2.1 High Resolution Spectrometer (HRS) . . . . . . . . . . . 38

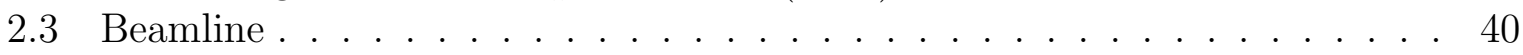

2.3.1 Beam position . . . . . . . . . . . . . . . . 40

2.3.2 Beam current . . . . . . . . . . . . . . . . . 41

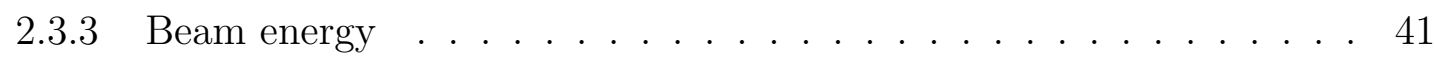

2.3.4 Polarimeters . . . . . . . . . . . . . . . . . . . 41

2.4 The target system . . . . . . . . . . . . . . . . . . . . . 43

2.5 Electromagnetic Calorimeter . . . . . . . . . . . . . . . . 43

2.6 Data acquisition . . . . . . . . . . . . . . . . . . . . . . . . . . . . . . . . . . . .

2.6.1 The Analog Ring Sampler . . . . . . . . . . . . . . . . . 45

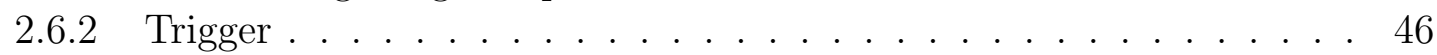

2.6.3 Data acquisition in Hall A . . . . . . . . . . . . . . . . . . . . 47

2.7 Experimental Setup . . . . . . . . . . . . . . . . . 48 
3 Calorimeter Signal Analysis and Calibration $\quad \mathbf{5 2}$

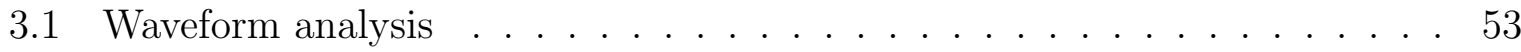

3.1.1 Reference pulses . . . . . . . . . . . . . . . 53

3.1.2 Baseline fit . . . . . . . . . . . . . . . . . . 54

3.1 .3 One pulse fit . . . . . . . . . . . . . . . . 55

3.1 .4 Two pulse fit . . . . . . . . . . . . . . . . . 56

3.1.5 Arrival time . . . . . . . . . . . . . . . . . . . 57

3.1.6 Analysis parameters . . . . . . . . . . . . . . . . . 59

3.2 Impact blocks selection, Clustering . . . . . . . . . . . . . . 62

3.2.1 Event time.......................... 64

3.2.2 Clustering thresholds . . . . . . . . . . . . . . . . 65

3.3 Calorimeter calibration . . . . . . . . . . . . . . . 66

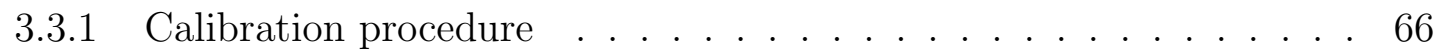

3.3.2 Calorimeter resolution . . . . . . . . . . . . . . . . 68

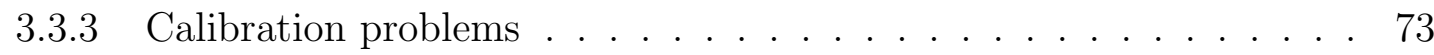

3.3.4 Coefficients comparison . . . . . . . . . . . . . . . . . 74

4 Background subtraction and extraction of observables $\quad 79$

4.1 Events selection . . . . . . . . . . . . . . . . . 79

4.1.1 Target, impact point . . . . . . . . . . . . . . . 80

4.1 .2 HRS efficiency . . . . . . . . . . . . . . . . . . 80

4.1 .3 HRS acceptance . . . . . . . . . . . . . . . . 82

4.1.4 Calorimeter, geometrical cut . . . . . . . . . . . . 84

4.2 Background subtraction . . . . . . . . . . . . . . . 85

4.2.1 $\pi^{0}$ subtraction . . . . . . . . . . . . . .... 85

4.2.2 Accidentals subtraction . . . . . . . . . . . . . . . . 89

4.3 Radiative corrections . . . . . . . . . . . . . . . . . . . . 91

4.4 Multicluster correction . . . . . . . . . . . . . . . . . . 93

4.5 Final data . . . . . . . . . . . . . . . . . . . . . . . 95

4.6 Systematic errors . . . . . . . . . . . . . . . . . . 96 96

4.7 Monte Carlo Simulation . . . . . . . . . . . . . . . . . . . . . 98

4.8 Extraction of observables . . . . . . . . . . . . . . . . . 100

4.8.1 Cross sections . . . . . . . . . . . . . . 103

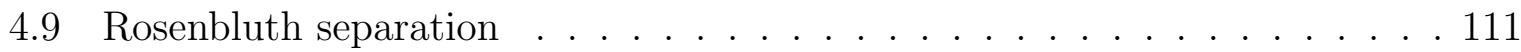

$\begin{array}{ll}\text { Conclusions } & 115\end{array}$

A Light cone coordinates $\quad 119$

$\begin{array}{ll}\text { B DVCS kinematics } & 121\end{array}$ 
C Coefficients and Compton form factors 123

C.1 Bethe-Heitler coefficients . . . . . . . . . . . . . . . . . 123

C.2 DVCS coefficients . . . . . . . . . . . . . . . . 124

C.3 Interference coefficients . . . . . . . . . . . . . . . . . . . . . 124

C.4 Compton form factors . . . . . . . . . . . . . . . . . . . 124

$\begin{array}{lr}\text { Bibliography } & 126\end{array}$

Resumen en español 134

$\begin{array}{ll}\text { Résumé en français } & 140\end{array}$ 



\section{Introduction}

An elementary particle is a particle whose substructure is unknown, thus it is unknown whether it is composed of other particles. Historically, the atom constituent particles (electrons, protons and neutrons) were all regarded as elementary particles. However, with the discovery of the magnetic moment of the proton [1], it became clear that the proton did not belong to this category. With further experimentation, more evidence was found that protons and neutrons, as well as all hadrons, were composed of other particles, and had an internal structure [2], [3]. The first evidence for quarks as real constituent elements of hadrons was obtained in late 1967, when the first of a long series of experiments on highly inelastic electron scattering was started at the two mile accelerator at the Stanford Linear Accelerator Center (SLAC) [4]. The raw counting rates were much higher than it had been expected in the deep inelastic region, where the electron imparts most of its energy to the proton. The experiment showed that the probability of deep inelastic scattering decreased much more slowly with the invariant momentum transfer to the proton, than that for elastic scattering [5]. A way to interpret this unexpected behavior was that the electrons were hitting some kind of hard core inside the target protons. The first evidence for gluons came in three jet events at PETRA [6].

In the summer of 1973 physicists at Harvard and Princeton demonstrated that in certain gauge theories the force between the quarks could become relatively weak at short distances, a behavior known as asymptotic freedom [7], which causes bonds between particles to become asymptotically weaker as energy increases and distance decreases. Another property of the interactions between quarks, known as confinement, states that the force between quarks does not diminish as they are separated. Because of this, when two quarks become separated, as happens in particle accelerator collisions, at some point it is more energetically favorable for a new quark-antiquark pair to spontaneously appear, than to allow the distance to extend further. Although analytically unproven, confinement is widely believed to be true because it explains the consistent failure of free quark searches. There is no known phase-transition line separating these two properties. Confinement is dominant in low-energy scales but, as energy increases, asymptotic freedom becomes 
dominant. These interactions between partons, quarks and gluons, are described by the quantum chromodynamics (QCD) theory.

Nowadays, Deep Inelastic Scattering (DIS) continues to offer a path to extract new information on the hadronic structure, and consequently, on the unknowns of how QCD works. The experiment this thesis is based on, Jefferson Lab E07-007 [8], seeks to exploit this kind of process, and more specifically, the Virtual Compton Scattering (VCS) process, which is an exclusive reaction of DIS. VCS consists in the production of a real photon off the nucleon, when scattered by a virtual photon,

$$
\gamma^{*}+p \rightarrow \gamma+p^{\prime}
$$

This kind of reactions, in a certain kinematic regime known as Deeply Virtual Compton Scattering (DVCS), can provide interesting information about a new class of quark and gluon matrix elements, called Generalized Parton Distributions (GPDs). GPDs correlate the transverse spatial distribution of the struck parton (quark or gluon) with the light-cone momentum fraction of the parton in the target. This provides us with information about spatial and momentum distributions of partons within the nucleon, which helps us understand how the behavior of partons conferes the characteristics of the nucleon.

Due to the small cross section of DVCS (of the order of $n b$ ), in order to conduct these kind of experiments it is necessary to make use of facilities capable of providing high beam intensities. One of these facilities is the Thomas Jefferson National Accelerator Facility [9], where the experiment JLab E07-007 [10], "Complete Separation of Virtual Photon and $\pi^{0}$ Electroproduction Observables of Unpolarized Protons", took place during the months of October to December of 2010. I started my collaboration with the experiment several months after the data acquisition. Here I present my work on the data analysis as well as the computed cross sections of the studied reaction.

- Chapter 1 is a theoretical introduction to the study of the nucleon structure, reviewing the concepts of form factors and parton distributions through elastic and inelastic processes. The computation of the photon leptoproduction cross section is described in detail, as well as the goals of experiment E07-007.

- Chapter 2 is a description of Jefferson Lab main characteristics, focusing on the experimental Hall A, where the experiment took place, and its instrumentation. The experimental setup along with the kinematics employed during data acquisition can be found in this chapter. Special detail is given to the electromagnetic calorimeter, the device on which most of the work of this thesis is based on. 
- Chapter 3 describes the analysis of the data stored by the electromagnetic calorimeter, with the purpose of obtaining the kinematic variables of the real photons resulting from DVCS reactions. This chapter also includes the process of calibration of this apparatus as well as the computation of its energy and angular resolution.

- Chapter 4 describes the selection of events from stored data, the applied cuts to kinematical variables and the background subtraction. Also, the process of extraction of the necessary observables for computing the photon leptoproduction cross section is described, along with the main steps followed to perform the Monte Carlo simulation used in this computation. The resulting cross sections are shown at the end of the chapter. 



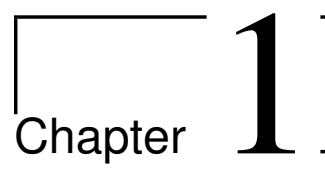

\section{Deeply Virtual Compton Scattering}

The study of the inner structure of hadrons can be tackled mainly through elastic or inelastic scattering. On one hand, elastic processes lead us to nucleon form factors, the modification of the cross section due to the finite size of nucleons. On the other hand, deeply inelastic processes (DIS) lead us to Parton Distribution Functions (PDFs), which in the framework of the parton model can be interpreted as probability densities of finding a parton with a certain longitudinal momentum fraction. Both approaches are complementary, but bear similar drawbacks. The form factors do not yield direct information about the momentum of the hadronic constituents, whereas the momentum distribution does not give information on their spatial location. Quantum-mechanically, the phase structure is totally washed out as well. If we want to unify both concepts (momentum and spatial distributions) we must resort to Generalized Parton Distributions (GPDs), and we do so by studying exclusive processes of DIS like Deeply Virtual Compton Scattering (DVCS). GPDs correlate the spatial distribution with the light-cone momentum fraction of partons within the nucleon.

The easiest way to access these GPDs is through DVCS. This process can be expressed as the scattering of an electron by a proton by means of a virtual photon with the result of the scattered initial particles plus a real photon,

$$
p+e \rightarrow p^{\prime}+e^{\prime}+\gamma
$$

In this theoretical introductory chapter, I will review elastic and inelastic scattering processes, how do they correlate to form factors and PDFs respectively, as well as introduce DVCS reactions and their linking to GPDs. Then I will move on to give the steps for the computation of the $\left(p+e \rightarrow p^{\prime}+e^{\prime}+\gamma\right)$ cross section, including the Bethe-Heitler contribution, in order to establish the relation between cross section and GPDs. Also the goal of the experiment JLab experiment E07-007 will be given through this description. 


\subsection{Elastic scattering: form factors}

Electron elastic scattering on the proton $\left(e+p \rightarrow e^{\prime}+p^{\prime}\right)$ provides us with information about charge and magnetism distribution and consequently about space distribution of the nucleon components. The mathematical tools employed in this study are the so called form factors, which relate our cross section to that of a point like charge.

In 1911 Rutherford developed a scattering formula in the limit where the target recoil is neglected and the scattered particle is non-relativistic. He did so by studying scattered alpha particles on a gold foil. But the formula works as well for electrons with a kinetic energy $E_{K} \ll m_{e}$. He wrote the cross section as [11]

$$
\left(\frac{d \sigma}{d \Omega}\right)_{\text {Rutherford }}=\frac{\alpha^{2}}{16 E_{K}^{2} \sin ^{4}\left(\frac{\theta}{2}\right)},
$$

where $\alpha$ is the fine-structure constant. For the relativistic case we have to turn to the so called Mott Scattering, where $E \gg m_{e}$. Here again the target recoil is neglected,

$$
\left(\frac{d \sigma}{d \Omega}\right)_{M o t t}=\frac{\alpha^{2}}{4 E^{2} \sin ^{4}\left(\frac{\theta}{2}\right)} \cos ^{2}\left(\frac{\theta}{2}\right) .
$$

The $\cos ^{2}(\theta / 2)$ term comes from the overlap between initial/final state electron wavefunctions with spin $1 / 2$. So far we have described the scattering cases for point like targets. If we consider the scattering of an electron due to an extended charge distribution (Fig. 1.1) our cross section will be modified by the form factor $F(\Delta)$ [12], which depends on the momentum transfer to the nucleon $\Delta=p-p^{\prime}$,

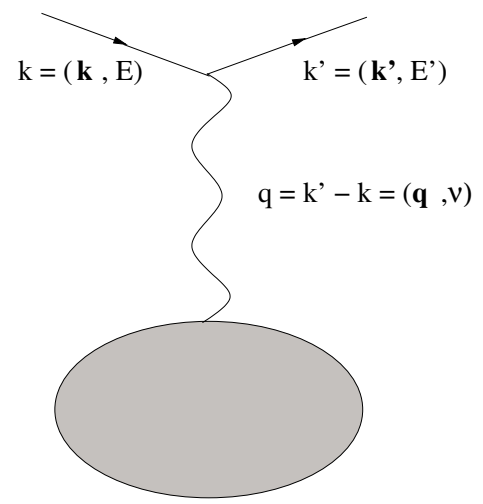

Figure 1.1: Scattering of an electron by a nucleon. The exchange of a photon is involved in the process.

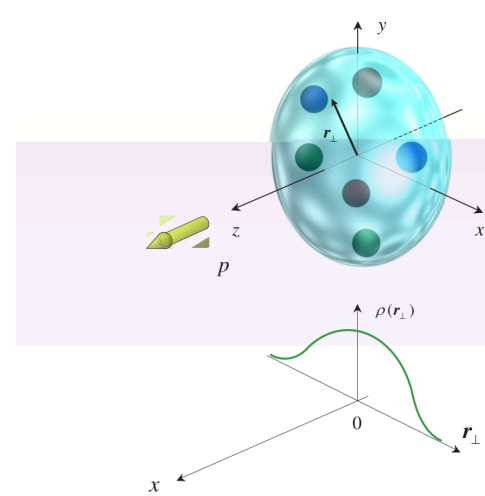

Figure 1.2: Nucleon charge distribution as a function of the nucleon radius in the infinite momentum frame $p_{z} \rightarrow \infty$. 


$$
\frac{d \sigma}{d \Omega}=\left(\frac{d \sigma}{d \Omega}\right)_{\text {point }}|F(\Delta)|^{2} \quad \longrightarrow \quad \frac{d \sigma}{d \Omega}=\frac{\alpha^{2}}{4 E^{2} \sin ^{4}\left(\frac{\theta}{2}\right)} \cos ^{2}\left(\frac{\theta}{2}\right)|F(\Delta)|^{2} .
$$

For unpolarized electrons on a static spinless target, the form factor is described as the Fourier transform of the charge distribution $\rho(r)$,

$$
F(\Delta)=\int \rho(r) e^{i \Delta r} d x^{3} \quad \text { with } \quad \int \rho(r) d r^{3}=1 .
$$

Therefore, by measuring the form factor we have access to the charge distribution (Fig. 1.2. Form factors can be defined by the matrix element between the nucleon states with different four-momenta of the quark electromagnetic or weak current. Using Lorentz invariant discrete symmetries and the spin $1 / 2$ nature of the proton, form factors (FFs) can be expressed in terms of vector and axial-vector QCD local operators in space-time coordinates as

$$
\begin{aligned}
\left\langle p^{\prime}\left|\bar{\psi}_{q}(0) \gamma^{\mu} \psi_{q}(0)\right| p\right\rangle & =F_{1}^{q}\left(\Delta^{2}\right) \bar{N}\left(p^{\prime}\right) \gamma^{\mu} N(p)+F_{2}^{q}\left(\Delta^{2}\right) \bar{N}\left(p^{\prime}\right) i \sigma^{\mu \nu} \frac{\Delta_{\nu}}{2 M_{N}} N(p), \\
\left\langle p^{\prime}\left|\bar{\psi}_{q}(0) \gamma^{\mu} \gamma_{5} \psi_{q}(0)\right| p\right\rangle & =G_{A}^{q}\left(\Delta^{2}\right) \bar{N}\left(p^{\prime}\right) \gamma^{\mu} \gamma_{5} N(p)+G_{P}^{q}\left(\Delta^{2}\right) \bar{N}\left(p^{\prime}\right) \gamma_{5} \frac{\Delta^{\mu}}{2 M_{N}} N(p) .
\end{aligned}
$$

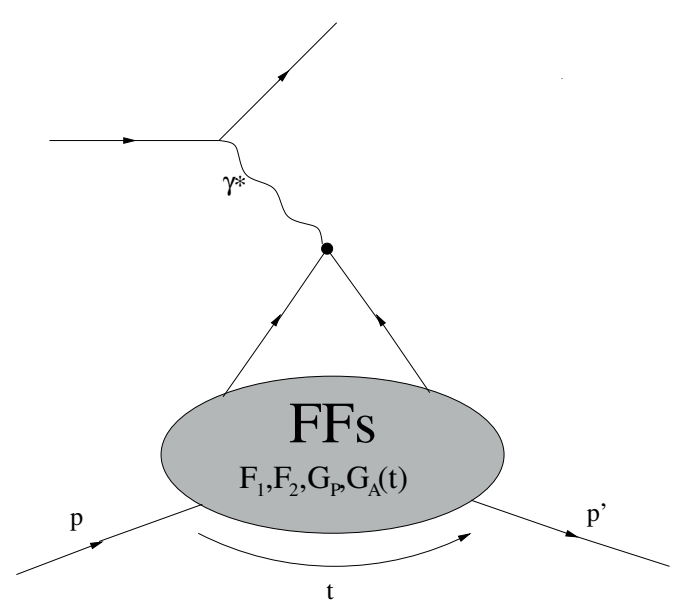

Figure 1.3: Elastic scattering, one parton interaction. $\mathrm{t}=\Delta^{2}$.

Where $F_{1}^{q}$ and $F_{2}^{q}$ are Dirac and Pauli form factors, and $G_{A}^{q}$ and $G_{P}^{q}$ are the axial and pseudoscalar form factors, respectively. Here, $p$ and $p^{\prime}$ are the initial and final hadron 
momentum and $\psi_{q}$ is the quark field of flavor $q$. For the case of the proton, the matrix element of the first equation in (1.6) is parametrized by two form factors, $F_{1}^{q}$ and $F_{2}^{q}$. At the same time one can parametrize the matrix element of the proton by two different form factors, one related to the charge distribution in the proton, $G_{E}\left(\Delta^{2}\right)$, and the other related to the distribution of the magnetic moment of the proton, $G_{M}\left(\Delta^{2}\right)$ which hold the following relationships with Dirac and Pauli form factors:

$$
\begin{aligned}
& G_{E}\left(\Delta^{2}\right)=F_{1}\left(\Delta^{2}\right)-\frac{\Delta^{2}}{4 M_{N}^{2}} F_{2}\left(\Delta^{2}\right), \\
& G_{M}\left(\Delta^{2}\right)=F_{1}\left(\Delta^{2}\right)+F_{2}\left(\Delta^{2}\right) .
\end{aligned}
$$

Taking the latter into account it can be shown that equation (1.3) generalizes to the Rosenbluth formula [13]:

$$
\frac{d \sigma}{d \Omega}=\frac{\alpha^{2}}{4 E^{2} \sin ^{4}\left(\frac{\theta}{2}\right)} \frac{E^{\prime}}{E}\left(\frac{G_{E}^{2}\left(\Delta^{2}\right)+\tau G_{M}^{2}\left(\Delta^{2}\right)}{(1+\tau)} \cos ^{2}\left(\frac{\theta}{2}\right)+2 \tau G_{M}^{2}\left(\Delta^{2}\right) \sin ^{2}\left(\frac{\theta}{2}\right)\right),
$$

with

$$
\tau=\frac{-\Delta^{2}}{4 M^{2}} .
$$

\subsection{Deeply Inelastic scattering: parton distributions}

Let us consider the inclusive process $e+N \rightarrow e^{\prime}+X$, in which an electron is scattered by a nucleon, resulting in a combination of final particles $(X)$. These processes provide us with information about parton momentum distribution within the nucleon.

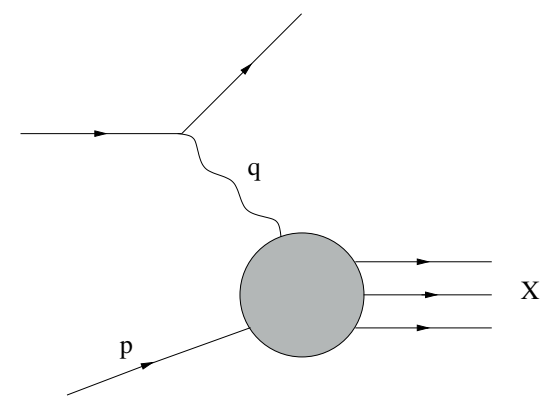

Figure 1.4: Deeply Inelastic scattering. 
In Deeply Inelastic Scattering (DIS) only the scattered electron is detected, therefore, there is some energy from the initial state that goes undetected at the final state. The invariant mass of the final hadronic state may be expressed as $W$ :

$$
W^{2}=\left(2 \nu+M^{2}-\mathcal{Q}^{2}\right) .
$$

Here we have the invariants $\mathcal{Q}^{2}=-q^{2}$ (the virtuality of the photon), and $\nu=p \cdot q$. In the reference frame where the initial hadron is at rest, $\nu=M\left(E^{\prime}-E\right)$, where $E^{\prime}$ and $E$ are the final and initial electron energy, and $M$ is the hadron's mass. In the case of the nucleon being a proton, we describe the process as follows: an initial electron $e(k)$ interacts with the proton in its initial state $|p\rangle$ by means of a virtual photon $\gamma^{*}(q)$. The result is a scattered electron $e\left(k^{\prime}\right)$ and the final hadronic $\langle X|$ state. The amplitude of this process is described by the current-to-current coupling [14],

$$
\mathcal{A}_{X}=L_{\mu}\left(k, k^{\prime}\right)\left\langle X\left|j^{\mu}(0)\right| p\right\rangle \quad \text { with } \quad L_{\mu}\left(k, k^{\prime}\right)=\frac{i}{q^{2}} \bar{u}\left(k^{\prime}\right) \gamma^{\mu} u(k) .
$$

Where $L_{\mu}\left(k, k^{\prime}\right)$ stands for the leptonic current. The scattered amplitude $\mathcal{A}_{X}$ for electronhadron scattering can be split into a leptonic tensor $L_{\mu}^{\dagger} L_{\nu}$ and a hadronic tensor $W^{\mu \nu}$, rendering the differential cross section for inclusive scattering with the form

$$
\sigma_{D I S}=\frac{\alpha_{e m}^{2}}{4 \pi} \sum_{n}\left|\mathcal{A}_{X}\right|^{2}(2 \pi)^{4} \delta^{(4)}\left(p+q-P_{X}\right)=\frac{\alpha_{e m}^{2}}{q^{4}} L_{\mu}^{\dagger} L_{\nu} W^{\mu \nu},
$$

where the hadronic tensor reads

$$
W^{\mu \nu}=\frac{1}{4 \pi} \int d z^{4} e^{i q z}\left\langle p\left|j^{\mu}(z) j^{\nu}(0)\right| p\right\rangle .
$$

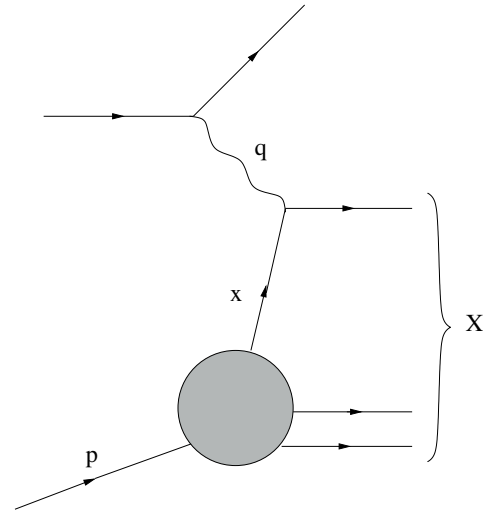

Figure 1.5: Deeply Inelastic Scattering. The virtual photon interacts with only one parton.

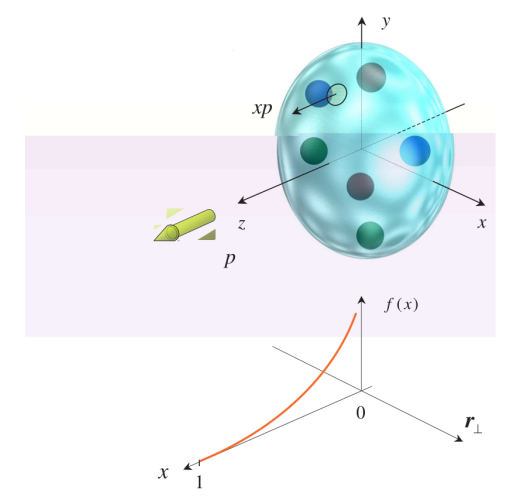

Figure 1.6: Probabilistic interpretation of a parton distribution in terms of the fraction of carried momentum $x$. 
In the case of the Bjorken limit $\mathcal{Q}^{2} \rightarrow \infty$ and $\nu \rightarrow \infty$ at fixed $x_{B}=\mathcal{Q}^{2} /(2 p \cdot q)$, it is possible to make the assumption that the photon is only interacting with one parton, putting forth the point like nature of quarks and gluons and giving us the possibility of describing the process by the diagram in Figure 1.5, while dismissing more complex diagrams. The diagram may be split into two parts. The first part involves the scattering of an electron and a parton, with a fraction moment $x$, through a virtual photon interaction, which is calculable through perturbative QCD, and through QED as well. The second one relates to the behavior of the parton within the hadron and is expressed through the unpolarized and polarized Parton Distribution Functions (PDFs, $f_{1}(x)$ and $\left.g_{1}(x)\right)$. It is possible to correlate the hadronic tensor to the imaginary part of the forward virtual Compton scattering amplitude $T^{\mu \nu}[15]$ by making use of the Optical Theorem. The amplitude of this exclusive process can be expressed in terms of the PDFs as well.

$$
W^{\mu \nu}=\frac{1}{2 \pi} \Im m T^{\mu \nu}
$$

where

$$
T^{\mu \nu}=i \int d^{4} z e^{i q \cdot z}\left\langle p\left|T\left\{j^{\mu}(z) j^{\nu}(0)\right\}\right| p\right\rangle
$$

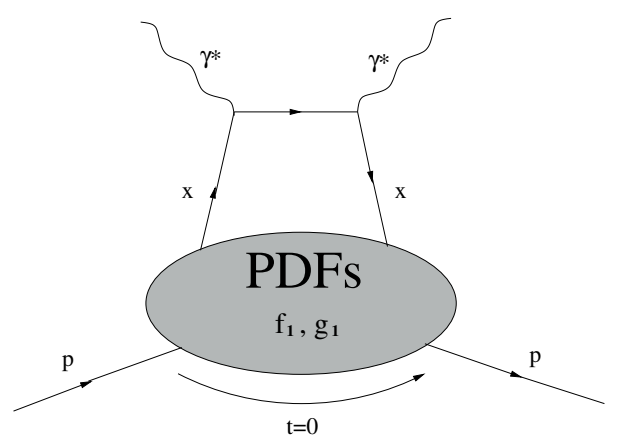

Figure 1.7: Forward Compton scattering. The virtual photon interacts with a parton carrying a momentum fraction $x$.

The points of absorption and emission in Figure 1.7, are separated by a light-like distance, where the use of light-cone coordinates proves useful. A detailed description of these coordinates can be found in Appendix A. To support the use of a single diagram we can calculate the probability for coherent scattering on an n-parton configuration [16],

$$
\mathcal{P} \sim\left(\frac{\left|\delta \mathbf{z}_{\perp}\right|^{2}}{\pi R_{N}^{2}}\right)^{n} \sim \frac{1}{\left(\mathcal{Q}^{2} \pi R_{N}^{2}\right)^{n}},
$$

where $\mathbf{z}_{\perp}$ is the transverse separation between the points of absorption and emission of a parton, in light-cone coordinates, and $\pi R_{N}^{2}$ is the transverse area of the nucleon. 
The probability is suppressed by the nth power of the photon virtuality, where powersuppressed corrections go under the name of higher twists. In a frame where the nucleon approaches the speed of light in a certain direction, $x$ is the longitudinal momentum fraction carried by the quark which is struck by the virtual photon. The PDFs represent therefore the (longitudinal) momentum distribution of quarks in the nucleon and give us the probability of finding a parton with a momentum fraction $x$ inside the hadron (Figure 1.6) [17]. The PDF structure functions correspond to QCD operators depending on space-time coordinates. Precisely, the PDFs are obtained as one-dimensional Fourier transforms in the light like coordinate $z^{-}$(at zero values of the other coordinates) as [14]:

$$
\begin{aligned}
& f_{1}^{q}(x)=\left.\frac{p^{+}}{4 \pi} \int d z^{-} e^{i x p^{+} z^{-}}\left\langle p\left|\bar{\psi}_{q}(0) \gamma^{+} \psi_{q}(z)\right| p\right\rangle\right|_{z^{+}=\vec{z}_{\perp}=0}, \\
& g_{1}^{q}(x)=\left.\frac{p^{+}}{4 \pi} \int d z^{-} e^{i x p^{+} z^{-}}\left\langle p S_{\|}\left|\bar{\psi}_{q}(0) \gamma^{+} \gamma_{5} \psi_{q}(z)\right| p S_{\|}\right\rangle\right|_{z^{+}=\vec{z}_{\perp}=0} .
\end{aligned}
$$

Where $S_{\|}$is the longitudinal nucleon spin projection. At this point we are able to relate our hadronic tensor to the PDFs, but first, we introduce the structure functions through the decomposition of the hadronic tensor in independent Lorentz tensors in a more detailed expression for (1.13). For the spin one-half target $W^{\mu \nu}$ can be expressed as [14]:

$$
\begin{aligned}
W^{\mu \nu} & =-\left(g^{\mu \nu}-\frac{q^{\mu} q^{\nu}}{q^{2}}\right) F_{1}\left(x_{B}, \mathcal{Q}^{2}\right)+\frac{1}{p \cdot q}\left(p^{\mu}-\frac{p \cdot q}{q^{2}} q^{\mu}\right)\left(p^{\nu}-\frac{p \cdot q}{q^{2}} q^{\nu}\right) F_{2}\left(x_{B}, \mathcal{Q}^{2}\right) \\
& -\frac{i}{p \cdot q} \varepsilon^{\mu \nu \rho \sigma} q_{\rho} s_{\sigma} g_{1}\left(x_{B}, \mathcal{Q}^{2}\right)-\frac{i}{p \cdot q} \varepsilon^{\mu \nu \rho \sigma} q_{\rho}\left(s_{\sigma}-\frac{s \cdot q}{p \cdot q} p_{\sigma}\right) g_{2}\left(x_{B}, \mathcal{Q}^{2}\right) .
\end{aligned}
$$

Using the latter expression we can also rewrite our cross section from (1.12) in terms of the structure functions. For the spin average case we have:

$$
\frac{d \bar{\sigma}_{D I S}^{3}}{d x_{B} d y d \Phi}=\frac{e^{4}}{4 \pi^{2} \mathcal{Q}^{2}}\left\{\frac{y}{2} F_{1}\left(x_{B}, \mathcal{Q}^{2}\right)+\frac{1}{2 x_{B} y}\left(1-y-\frac{y^{2} 4 x_{B}^{2} M^{2}}{\mathcal{Q}^{2}}\right) F_{2}\left(x_{B}, \mathcal{Q}^{2}\right)\right\}
$$

where $y=\mathcal{Q}^{2} / 2 M E x_{B}$ and $\Phi$ is the azimuthal angle between the plane defined by $k$ and $k^{\prime}$, and the plane defined by $k$ and $S$. There is no significant dependence on $\Phi$, which cannot even be uniquely defined for inclusive scattering with an unpolarized target. In the Bjorken limit, the structure functions are expressed in terms of quark distributions. For instance, the proton polarization-independent structure functions are:

$$
\begin{aligned}
F_{1}\left(x_{B}, \mathcal{Q}^{2}\right) & =\frac{1}{2 x_{B}} F_{2}\left(x_{B}, \mathcal{Q}^{2}\right) \\
& =\frac{1}{2} \sum_{q} Q_{q}^{2}\left(q\left(x_{B}, \mathcal{Q}^{2}\right)+\bar{q}\left(x_{B}, \mathcal{Q}^{2}\right)\right)=\frac{1}{2} \sum_{q} Q_{q}^{2} f_{1}^{q}\left(x_{B}, \mathcal{Q}^{2}\right),
\end{aligned}
$$


where $Q_{q}$ is the quark charge. We introduced, in eq. (1.20) the spin independent parton distributions $q(x)=f_{1}^{q}(x)$ for $x>0$, and $\bar{q}(x)=-f_{1}^{q}(-x)$ for $x<0$ (antiquarks). This way, through measuring the structure functions in (1.19) it is possible to have access to the PDFs. Analogous relations hold for the polarized structure function $g_{1}^{q}\left(x_{B}, \mathcal{Q}^{2}\right)$, the only difference is that spin-weighted rather than spin averaged quark parton species enter the leading order prediction,

$$
g_{1}^{q}\left(x_{B}, \mathcal{Q}^{2}\right)=\frac{1}{2} \sum_{q} Q_{q}^{2}\left(\Delta q\left(x_{B}, \mathcal{Q}^{2}\right)+\Delta \bar{q}\left(x_{B}, \mathcal{Q}^{2}\right)\right)=\frac{1}{2} \sum_{q} Q_{q}^{2} g_{1}^{q}\left(x_{B}, \mathcal{Q}^{2}\right)
$$

\subsection{Exclusive reactions: DVCS}

We have seen how to access parton space distributions through elastic scattering on one side, and how to access parton momentum distributions through deeply inelastic scattering on the other side. However all this information is not sufficient to describe the hadronic wave function in detail. To do so we need to study specific cases of DIS (exclusive reactions) which will help us correlate both kinds of distributions. Deeply Virtual Compton Scattering is one of them (Figure 1.8).

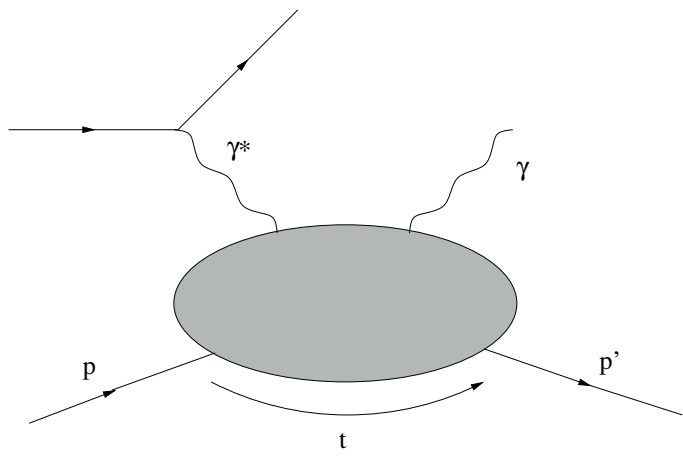

Figure 1.8: Deeply Virtual Compton Scattering. A real photon is emitted as a result of the scattering.

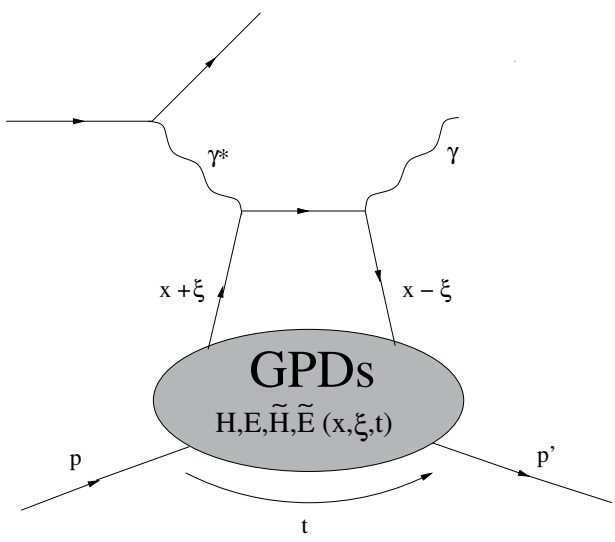

Figure 1.9: Handbag diagram for DVCS. The virtual photon interacts with only one parton.

This scattering process depends mainly on 3 different kinematic variables: the interaction photon virtuality $\mathcal{Q}^{2}$, its energy at the lab frame $\nu$, and the transferred momentum $t=\Delta^{2}$ (with $\Delta_{\mu}=\left(p-p^{\prime}\right)_{\mu}$ ). Since this thesis is based on JLab experiment E07-007, where the reaction $e+p \rightarrow e^{\prime}+p^{\prime}+\gamma$ was studied, we will refer to that process from now on. In a similar way as we previously did with DIS, at fixed $x_{B}$ but $\mathcal{Q}^{2}$ and $\nu$ high enough we make the assumption that the virtual photon interacts with a single quark (Handbag 
approximation), this time carrying a momentum fraction $x+\xi$ (Figure 1.9), which is the light-cone + momentum fraction. The quark returns to the nucleon carrying a light-cone + momentum fraction $x-\xi$.

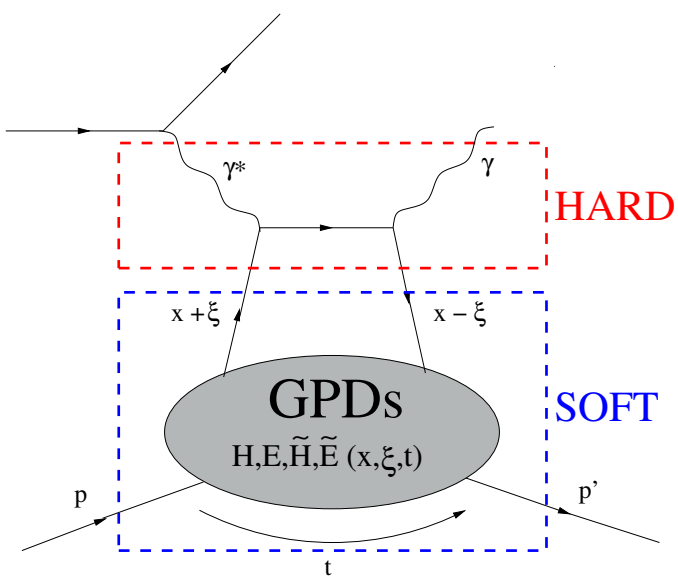

Figure 1.10: Handbag diagram. The hard part is calculable through perturbative QCD, and the soft part is described in terms of GPDs.

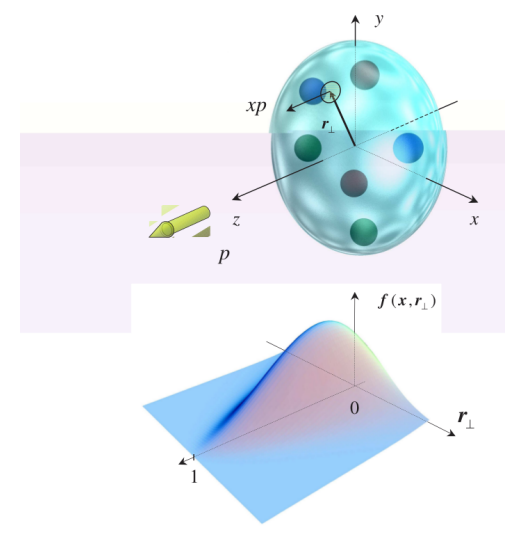

Figure 1.11: Probabilistic interpretation of a Generalized Parton Distribution in terms of the fraction of carried momentum $x$ and the nucleon radius.

Figure 1.9 is the leading order diagram. We can as well split the diagram into two sections (Figure 1.10): the interaction of the scattered electron with a parton (HARD), calculable through perturbative QCD, and the parton interaction with the proton (SOFT), described in terms of GPDs. The concept of GPDs was developed as a modern tool to deliver a detailed description of the microscopic structure of hadrons in terms of their elementary constituents [18], [19], [20], [21], [22], [23]. This field has been very active in the last fifteen years, and there are several reviews discussing different aspects of the GPDs [24], [25], [26], [27], [28], [29], [30], [31], but from now on we will follow the description from [14], unless noted otherwise. Figure 1.11 shows their probabilistic interpretation in the infinite momentum frame $p_{z} \rightarrow \infty$. The longitudinal momentum fraction $x$ takes values between 0 and 1 along the $\mathrm{z}$ axis, while the charge distribution is shown as a function of $r_{\perp}$. GPDs correspond to the Fourier transform of QCD non-local and non-diagonal operators. "Non-local" since the initial and final quarks are created (or annihilated) at different same space-time points and "non-diagonal" since the momenta of the initial and final nucleons are different. Thus, in terms of the vector and axial-vector bilocal quark currents, GPDs can be expressed as

$$
\begin{aligned}
& \left.\frac{P^{+}}{4 \pi} \int d z^{-} e^{i x P^{+} z^{-}}\left\langle p^{\prime}\left|\bar{\psi}_{q}(0) \gamma^{+} \psi_{q}(z)\right| p\right\rangle\right|_{z^{+}=\vec{z}_{\perp}=0} \\
& =H^{q}(x, \xi, t) \bar{N}\left(p^{\prime}\right) \gamma^{+} N(p)+E^{q}(x, \xi, t) \bar{N}\left(p^{\prime}\right) i \sigma^{+\nu} \frac{\Delta_{\nu}}{2 M_{N}} N(p)
\end{aligned}
$$




$$
\begin{aligned}
& \left.\frac{P^{+}}{4 \pi} \int d z^{-} e^{i x P^{+} z^{-}}\left\langle p^{\prime}\left|\bar{\psi}_{q}(0) \gamma^{+} \gamma^{5} \psi_{q}(z)\right| p\right\rangle\right|_{z^{+}=\vec{z}_{\perp}=0} \\
& =\widetilde{H}^{q}(x, \xi, t) \bar{N}\left(p^{\prime}\right) \gamma^{+} \gamma_{5} N(p)+\widetilde{E}^{q}(x, \xi, t) \bar{N}\left(p^{\prime}\right) \gamma_{5} \frac{\Delta^{+}}{2 M_{N}} N(p) .
\end{aligned}
$$

Here $P$ is the sum of the nucleon initial and final momentum: $P=\left(p+p^{\prime}\right)$. GPDs $H$ and $E$ correspond with averages over the quark helicity. They are therefore called unpolarized GPDs. The GPDs $\widetilde{H}$ and $\widetilde{E}$ involve differences of quark helicities and are called polarized GPDs. At the nucleon level, $E$ and $\widetilde{E}$ are associated to a flip of the nucleon spin while $H$ and $\widetilde{H}$ leave it unchanged (Figure 1.12). The four GPDs therefore reflect the four independent helicity-spin combinations of the quark-nucleon system (conserving quark helicity). GPDs depend on additional variables compared to PDFs and FFs and they are a richer source of nucleon structure information.

- In the limit where $t \rightarrow 0$ and $\xi=0$ in equations (1.22) and (1.23), we retrieve the spin independent or spin dependent parton distributions $q(x), \Delta q(x)$, and their counterparts for antiquarks $\bar{q}(x)$ and $\Delta \bar{q}(x)$ :

$$
\begin{array}{lll}
H^{q}(x, \xi=0, t=0)=q(x) & \widetilde{H}^{q}(x, \xi=0, t=0)=\Delta q(x) & \text { for } x>0, \\
H^{q}(x, \xi=0, t=0)=-\bar{q}(-x) & \widetilde{H}^{q}(x, \xi=0, t=0)=\Delta \bar{q}(-x) & \text { for } x<0 .
\end{array}
$$

Since the defining equations of $E$ and $\widetilde{E}$ are proportional to $\Delta_{\mu}$ they decouple in the forward limit, and therefore, there are no analogous relations to those of $H$ and $\widetilde{H}$ with the quark distributions.

- We can also retrieve the FFs by forming the first moment of GPDs. By integrating equations (1.22) and (1.23) over $x$ we can correlate the results to equations in (1.6):

$$
\begin{aligned}
& \int_{-1}^{1} H^{q}(x, \xi, t) d x=F_{1}^{q}(t) \quad \int_{-1}^{1} E^{q}(x, \xi, t) d x=F_{2}^{q}(t) \quad \forall \xi \\
& \int_{-1}^{1} \widetilde{H}^{q}(x, \xi, t) d x=G_{A}^{q}(t) \quad \int_{-1}^{1} \widetilde{E}^{q}(x, \xi, t) d x=G_{P}^{q}(t)
\end{aligned}
$$

At $\xi=0$, the GPD (x, $0, \mathrm{t})$ can then be interpreted as the probability of finding a parton with longitudinal momentum fraction $\mathrm{x}$ at a given transverse distance (relative to the transverse c.m.) in the nucleon. In this way, the information contained 
in a traditional parton distribution, as measured in DIS, and the information contained within a form factor, as measured in elastic lepton-nucleon scattering, are combined and correlated in the GPD description.
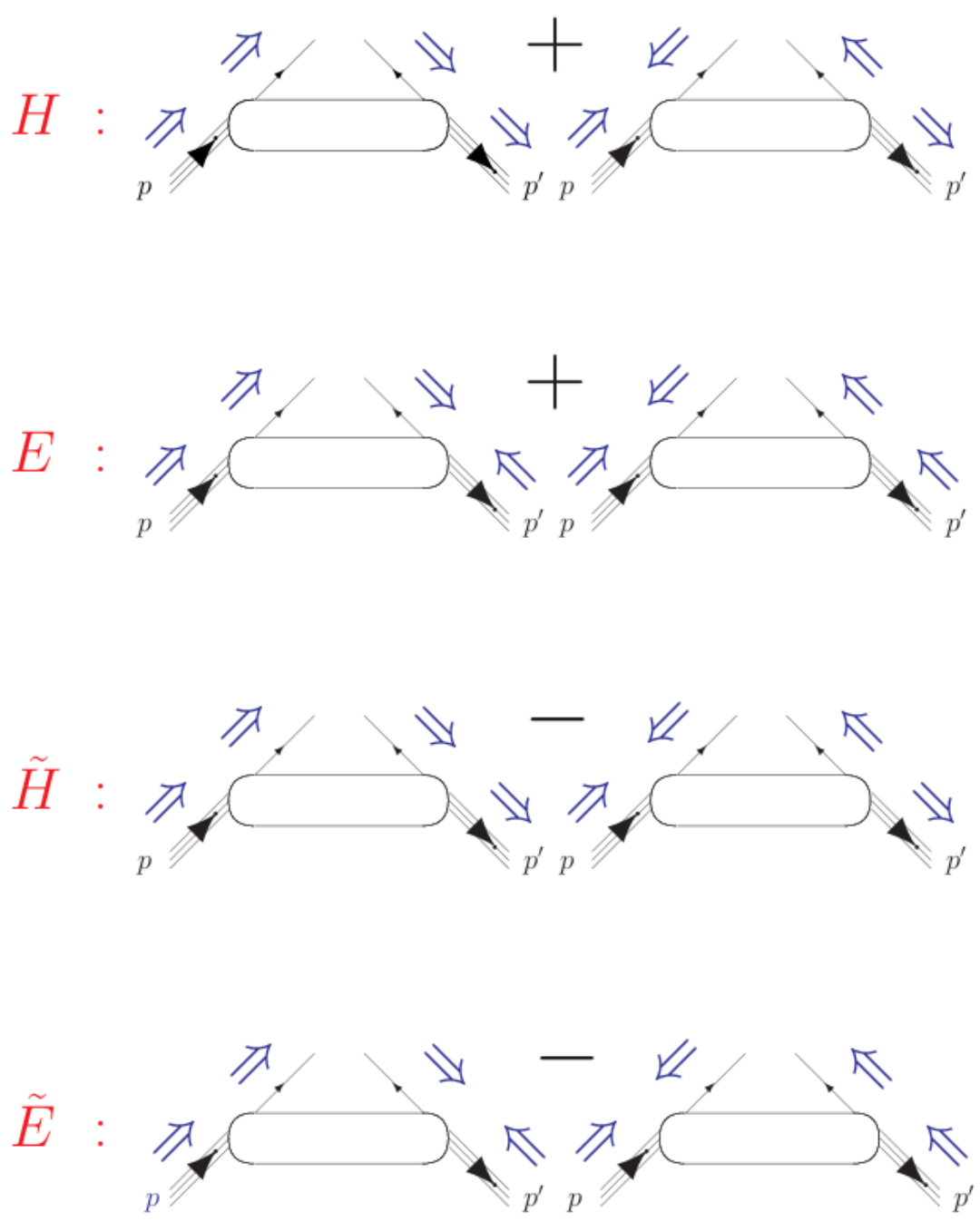

Figure 1.12: GPDs and their correspondence to quarks helicity and nucleon spin orientations.

- The second moment of the GPDs is relevant to the nucleon spin structure. It is known as Ji's sum rule [32] and reads:

$$
\frac{1}{2} \int_{-1}^{1}\left[H^{q}(x, \xi, 0)+E^{q}(x, \xi, 0)\right] x d x=J^{q} \quad \forall \xi
$$


There exists a (color) gauge-invariant decomposition of the nucleon spin: $1 / 2=$ $J_{q}+J_{g}$, where $J_{q}$ and $J_{g}$ are respectively the total quark and gluon contributions to the nucleon total angular momentum. The total quark spin contribution $J_{q}$ decomposes (in a gauge invariant way) as $J_{q}=1 / 2 \Delta \Sigma+L_{q}$ where $1 / 2 \Delta \Sigma$ and $L_{q}$ are respectively the quark spin and quark orbital contributions to the nucleon spin. $\Delta \Sigma$ can be measured through polarized DIS experiments. On the other hand, for the gluons it is still an open question how to decompose the total angular momentum $J_{g}$ into orbital angular momentum, $L_{g}$, and gluon spin, $\Delta g$, parts, in such a way that both can be related to observables. The sum rule of equation (1.27) in terms of the GPDs provides a model independent way of determining the quark orbital contribution to the nucleon spin and therefore completes the quark sector of the "spin-puzzle".

Below there is a summary of the operators and their associated structure functions we have seen so far, including FFs, PDFs and GPDs [33]:

\begin{tabular}{|c|c|c|}
\hline $\begin{array}{c}\text { Operator } \\
\text { in coordinate space }\end{array}$ & $\begin{array}{c}\text { Nature of the } \\
\text { matrix element }\end{array}$ & $\begin{array}{c}\text { Associated structure functions } \\
\text { in momentum space }\end{array}$ \\
\hline$\langle p|\bar{\psi}(0) \mathcal{O} \psi(z)| p\rangle$ & non-local, diagonal & $f_{1}(x), g_{1}(x)$ \\
\hline$\left\langle p^{\prime}|\bar{\psi}(0) \mathcal{O} \psi(0)| p\right\rangle$ & local, non-diagonal & $F_{1}(t), F_{2}(t), G_{P}(t), G_{A}(t)$ \\
\hline$\left\langle p^{\prime}|\bar{\psi}(0) \mathcal{O} \psi(z)| p\right\rangle$ & non-local, non-diagonal & $H(x, \xi, t), E(x, \xi, t), \widetilde{H}(x, \xi, t), \widetilde{E}(x, \xi, t)$ \\
\hline
\end{tabular}

Table 1.1: Operators and structure functions. $\mathcal{O}=\gamma^{+}$or $\gamma^{+} \gamma^{5}$.

\subsection{Deeply photon electroproduction cross section}

We describe the differential cross section of the exclusive leptoproduction of a photon, in the scattering of an electron by a proton. We follow the convention of [34]. The cross section is written as a function of the final states and including the energy-momentum conservation in the $\delta^{(4)}$ term,

$$
d \sigma=|\mathcal{T}|^{2} \frac{2 M 2 m_{e}}{4 \vec{p} \vec{k}} \frac{M d^{3} \overrightarrow{p^{\prime}}}{E^{\prime}(2 \pi)^{3}} \frac{m_{e} d^{3} \overrightarrow{k^{\prime}}}{E_{k^{\prime}}(2 \pi)^{3}} \frac{d^{3} \overrightarrow{q^{\prime}}}{2 E_{q^{\prime}}(2 \pi)^{3}}(2 \pi)^{4} \delta^{4}\left(\vec{p}+\vec{k}-\overrightarrow{p^{\prime}}-\overrightarrow{k^{\prime}}-\overrightarrow{q^{\prime}}\right) .
$$

Where $\vec{p}, \vec{k}, \overrightarrow{p^{\prime}}, \overrightarrow{k^{\prime}}$ and $\overrightarrow{q^{\prime}}$ stand for the incoming and scattered proton $\left(p, p^{\prime}\right)$ and electron $\left(k, k^{\prime}\right)$, and the real photon $\left(q^{\prime}\right)$ four-momenta. $|\mathcal{T}|$ stands for the total contribution of the different amplitudes of processes involved in leptoproduction of a photon from the nucleon. We now briefly discuss the computation of (1.28) phase space and $|\mathcal{T}|$. 


\subsubsection{Phase space}

Our cross section is defined in the laboratory frame where $\vec{p}=(M, 0,0,0)$ and $\vec{k}=$ $\left(E_{b}, 0,0, E_{b}\right)\left(\vec{k}\right.$ is in the $\vec{z}$ axis). We can use the $\delta^{(3)}$ in momentum to integrate over $q^{\prime}$,

$d \sigma=|\mathcal{T}|^{2} \frac{\left(2 M 2 m_{e}\right)^{2}}{16 M E} \frac{d^{3} \overrightarrow{p^{\prime}}}{E^{\prime}(2 \pi)^{3}} \frac{d^{3} \overrightarrow{k^{\prime}}}{E_{k^{\prime}}(2 \pi)^{3}} \frac{1}{E_{q^{\prime}}} \pi \delta\left(M+E-\sqrt{M^{2}+p^{2}}-\left|\overrightarrow{k^{\prime}}\right|-\sqrt{\left|\vec{p}+\vec{k}-\overrightarrow{p^{\prime}}-\overrightarrow{k^{\prime}}\right|^{2}}\right)$.

If we introduce the polar and azimuthal angles, $\bar{\theta}$ and $\bar{\phi}$, in our differential cross section, equation (1.29) will now depend on the variables $E^{\prime}, \cos \left(\bar{\theta}_{p^{\prime}}\right), \bar{\phi}_{p^{\prime}}$ and $k^{\prime}, \cos \left(\bar{\theta}_{k^{\prime}}\right), \bar{\phi}_{k^{\prime}}$, where the overline indicates they refer to the frame in which $\vec{k}=\left(E_{b}, 0,0, E_{b}\right)$. We can use the last $\delta$ to integrate over $\bar{\phi}_{p^{\prime}}$,

$$
\frac{d \sigma}{d E^{\prime} d k^{\prime} d \cos \left(\bar{\theta}_{p^{\prime}}\right) d \cos \left(\bar{\theta}_{k^{\prime}}\right) d \bar{\phi}_{k^{\prime}}}=|\mathcal{T}|^{2} \frac{\left(2 M 2 m_{e}\right)^{2}}{32 M E} \frac{1}{(2 \pi)^{5}} \frac{1}{\sin \left(\bar{\theta}_{p^{\prime}}\right) \sin \left(\bar{\phi}_{p^{\prime}}\right) \sin \left(\bar{\theta}_{k^{\prime}}\right)} .
$$

A rotation around the $\vec{y}$ axis must be applied in order to express (1.30) in the reference frame described by A.V. Belitsky, D. Müller and A. Kirchner in [35], in which $\overrightarrow{q_{1}}$ goes along the $\vec{z}$ axis. This frame, shown in Fig. 1.13, is employed to perform the decomposition in Fourier harmonics of the photon electroproduction.

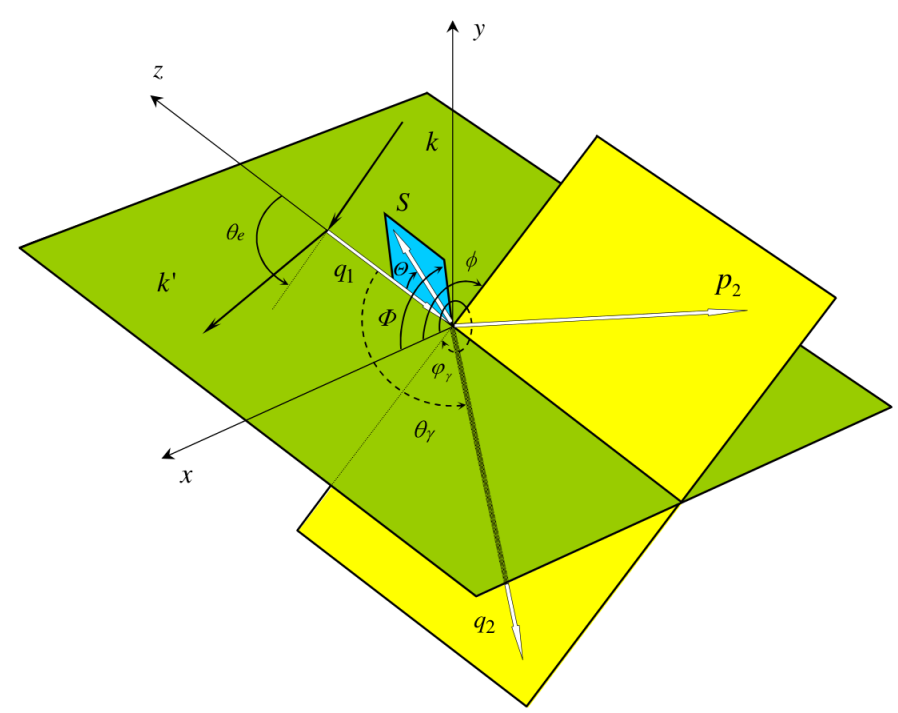

Figure 1.13: Kinematics of leptoproduction in the target rest frame.

The z-direction is chosen counter-along the three-momentum of the incoming virtual photon. The leptons three-momenta form the lepton scattering plane, while the recoiled 
proton and outgoing real photon define the hadron scattering plane. In this reference system $\phi$ is the azimuthal angle between the lepton plane and the recoiled proton momentum, and it is related in turn to the photon azimuthal angle $\varphi_{\gamma}=\phi+\pi$. The target polarization three-vector is described by two spherical angles $(\Theta, \Phi)$, so the angle between the polarization vector and the scattered nucleon is $\varphi=\Phi-\phi$. After applying the rotation we can rewrite our differential cross section to make it depend on the variables $x_{B}, y,\left|\Delta^{2}\right|, \phi$ and $\varphi$, instead of $E^{\prime}, k^{\prime}, \cos \left(\bar{\theta}_{p^{\prime}}\right), \cos \left(\bar{\theta}_{k^{\prime}}\right)$ and $\bar{\phi}_{k^{\prime}}$ (taking into account the relationship $\bar{\phi}_{k^{\prime}}=\phi_{k^{\prime}}=\varphi$ ). Then one gets to the five-fold cross section,

$$
\frac{d \sigma}{d x_{B} d y d\left|\Delta^{2}\right| d \phi d \varphi}=\frac{\alpha^{3} x_{B} y}{16 \pi^{2} \mathcal{Q}^{2} \sqrt{1+\varepsilon^{2}}}\left|\frac{\left(2 M 2 m_{e}\right) \mathcal{T}}{e^{3}}\right|^{2} \quad \text { with } \quad \varepsilon=\frac{2 x_{B} M}{\mathcal{Q}}
$$

Apart from the Bjorken variable $x_{B}$, the $t$-channel momentum transfer $\Delta^{2}$, and the lepton energy loss $y$, the differential cross section (1.31) depends on the azimuthal angles $\phi$ and $\varphi$.

\subsubsection{Contributing amplitudes}

In the energy range of Hall A electron beam, we find a process competing with DVCS known as Bethe-Heitler (BH). Figure 1.14 illustrates the main contributing diagrams to $|\mathcal{T}|$. The first diagram corresponds to Deeply Virtual Compton Scattering, in which the real and the virtual photons couple to quarks in the nucleon, its amplitude being $\mathcal{T}_{D V C S}$. The other two diagrams come from the "interfering" $\mathrm{BH}$ process, in which the real photon is radiated by the lepton rather than the quark. The $\mathrm{BH}$ amplitude will be denoted by $\mathcal{T}_{B H}$. The superposition principle leads to the presence of an interference term, $\mathcal{I}$, between these amplitudes in addition to the intensities $\left|\mathcal{T}_{D V C S}\right|^{2}$ and $\left|\mathcal{T}_{B H}\right|^{2}$,

$$
|\mathcal{T}|^{2}=\left|\mathcal{T}_{B H}\right|^{2}+\left|\mathcal{T}_{D V C S}\right|^{2}+\mathcal{I}
$$

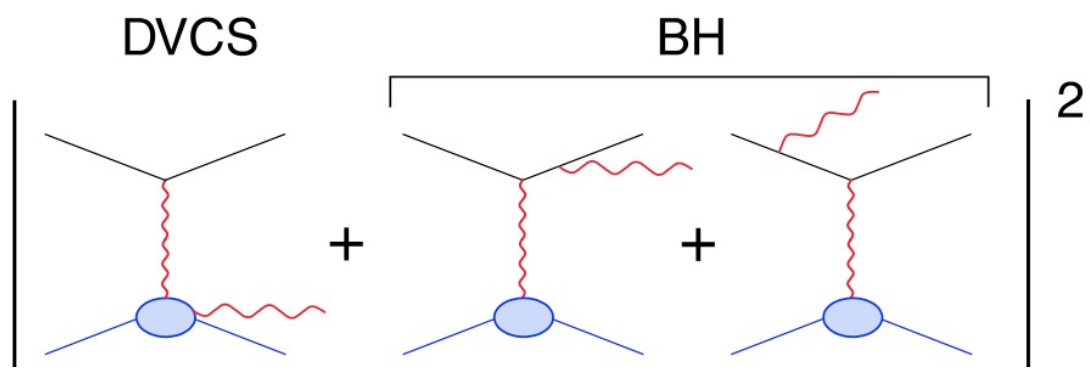

Figure 1.14: Reactions involved in the process (ep $\rightarrow e p \gamma)$. 


$$
\mathcal{I}=\mathcal{T}_{D V C S}^{*} \mathcal{T}_{B H}+\mathcal{T}_{D V C S} \mathcal{T}_{B H}^{*} .
$$

It is possible to exploit the structure of the cross section as a function of the angle $\phi$ between the leptonic and hadronic planes, to separate up to a certain degree the different contributions. In the following subsections, I will review the computation of the different amplitudes and their decomposition in Fourier harmonics depending on $\phi$.

\section{Bethe-Heitler amplitude}

In the $\mathrm{BH}$ process the resulting photon is emitted either by the incoming or the scattered electron. The BH amplitude is real (to the lowest order in the QED fine structure constant), and is parametrized in terms of electromagnetic form factors, and reads

$$
\mathcal{T}_{B H}=\frac{e^{3}}{\Delta^{2}} \varepsilon^{* \mu}\left(q_{2}\right) \bar{u}\left(k^{\prime}\right)\left(\gamma_{\mu} \frac{1}{\not k-\not} \gamma_{\nu}+\gamma_{\nu} \frac{1}{\not k^{\prime}+\not \Delta} \gamma_{\mu}\right) u(k) J^{\nu},
$$

where $J^{\nu}=\left\langle p^{\prime}\left|\bar{\psi}_{q}(0) \gamma^{\nu} \psi_{q}(0)\right| p\right\rangle$ is given in (1.6). The contribution to the cross section from $\mathrm{BH}$ comes from multiplying expression (1.34) by its conjugate. We write this contribution with its explicit dependence on the leptonic and hadronic tensors as:

$$
\left|\left(2 M_{p} 2 m_{e}\right) \mathcal{T}_{B H}\right|^{2}=\sum_{S} \frac{\left(2 M_{p} 2 m_{e}\right)^{2} e^{6}}{\Delta^{4}}\left(-g^{\mu \mu^{\prime}}\right) L_{\mu \nu}^{B H} L_{\mu^{\prime} \nu^{\prime}}^{\dagger B H} J^{\nu} J^{\dagger \nu^{\prime}},
$$

where we sum over initial and final spin states $S$. In order to decompose our amplitude in Fourier harmonics, we manipulate the expressions for the leptonic and hadronic tensors and rewrite the latter one in a way such as all the harmonics will have a similar dependence with the mentioned form factors:

$$
\begin{array}{r}
\sum_{S} \frac{\left(2 m_{e}\right)^{2} e^{6}}{\Delta^{4}}\left(-g^{\mu \mu^{\prime}}\right) L_{\mu \nu}^{B H} L_{\mu^{\prime} \nu^{\prime}}^{\dagger B H}=\frac{e^{6}}{4 \Delta^{4}} \operatorname{Tr}\left[\not k^{\prime}\left(\gamma_{\mu} \frac{\not k-\Delta}{\Delta^{2}-2 k \cdot \Delta} \gamma_{\nu}+\gamma_{\nu} \frac{\not k^{\prime}+\Delta}{\mathcal{Q}^{2}+2 k \cdot \Delta} \gamma_{\mu}\right)\right. \\
\left.\not k\left(\gamma_{\nu^{\prime}} \frac{\not k-\Delta}{\Delta^{2}-2 k \cdot \Delta} \gamma^{\mu}+\gamma^{\mu} \frac{\not k^{\prime}+\Delta}{\mathcal{Q}^{2}+2 k \cdot \Delta} \gamma_{\nu^{\prime}}\right)\right],
\end{array}
$$

$$
\begin{aligned}
\sum_{S}\left(2 M_{p}\right)^{2} J^{\nu} J^{\dagger \nu^{\prime}} & =\frac{\Delta^{2}}{2} g^{\nu \nu^{\prime}}\left(F_{1}+F_{2}\right)^{2}+\frac{1}{2}\left(p^{\nu} p^{\nu^{\prime}}+p^{\prime \nu} p^{\prime \nu^{\prime}}\right)\left[\left(F_{1}^{2}-\frac{\Delta^{2}}{4 M_{p}^{2}} F_{2}^{2}\right)-\left(F_{1}+F_{2}\right)^{2}\right] \\
& +\frac{1}{2}\left(p^{\nu} p^{\prime \nu^{\prime}}+p^{\prime \nu} p^{\nu^{\prime}}\right)\left[\left(F_{1}^{2}-\frac{\Delta^{2}}{4 M_{p}^{2}} F_{2}^{2}\right)+\left(F_{1}+F_{2}\right)^{2}\right] .
\end{aligned}
$$


The $\phi$ dependence of the amplitude comes from the term $k \cdot \Delta$, which is related to $\cos (\phi)$. The expression for this term as well as those for the whole kinematics used in these calculations can be found in Appendix B. Finally, the decomposition of the BH amplitude in Fourier harmonics has the expression [16]:

$\left|\left(2 M_{p} 2 m_{e}\right) \mathcal{T}_{B H}\right|^{2}=\frac{e^{6}}{x_{B}^{2} y^{2}\left(1+\varepsilon^{2}\right)^{2} \Delta^{2} \mathcal{P}_{1}(\phi) \mathcal{P}_{2}(\phi)}\left\{c_{0}^{B H}+\sum_{n=1}^{2} c_{n}^{B H} \cos (n \phi)+s_{n}^{B H} \sin (\phi)\right\}$,

where $\mathcal{P}_{1}$ and $\mathcal{P}_{2}$ are related to the propagators appearing in the $\mathrm{BH}$ process:

$$
\begin{aligned}
& \mathcal{Q}^{2} \mathcal{P}_{1}=\left(k-q_{2}\right)^{2}=\mathcal{Q}^{2}+2 k \cdot \Delta, \\
& \mathcal{Q}^{2} \mathcal{P}_{2}=(k-\Delta)^{2}=\Delta^{2}-2 k \cdot \Delta .
\end{aligned}
$$

It is possible to obtain $c_{0}^{B H}, c_{1}^{B H}$ and $c_{2}^{B H}$ by multiplying (1.36) by (1.37), then identifying those terms proportional to $\cos (\varphi)$ and $\cos ^{2}(\varphi) \cdot s_{1}^{B H}$ and $s_{2}^{B H}$ appear when we include helicity operators. The coefficients of the Fourier decomposition for this term as well as for the DVCS and interference terms are listed in Appendix C.

\section{DVCS amplitude}

The DVCS amplitude corresponds to the diagram depicted in figure 1.10, and is expressed in terms of the hadronic tensor $T^{\mu \nu}$. This tensor is given by the time-ordered product of the electromagnetic currents $j_{\mu}=e \Sigma_{q} Q_{q} \bar{\psi}_{q} \gamma^{\mu} \psi_{q}$ of quarks, having fractional charge $Q_{q}$, which is sandwiched between hadronic states with different momenta. The amplitude reads:

$$
\mathcal{T}_{D V C S}=\frac{e^{3}}{q_{1}^{2}} \varepsilon_{\mu}^{*}\left(q_{2}\right) \bar{u}\left(k^{\prime}\right) \gamma_{\nu} u(k) T^{\mu \nu},
$$

where we express $T^{\mu \nu}$ as [16]:

$$
\begin{aligned}
T^{\mu \nu} & =-\mathcal{D}^{\mu \rho} g_{\rho \sigma} \mathcal{D}^{\sigma \nu} \mathcal{F}_{1}+\frac{1}{P \cdot\left(q+q^{\prime}\right)} \mathcal{D}^{\mu \rho} P_{\rho} P_{\sigma} \mathcal{D}^{\sigma \nu} \mathcal{F}_{2}+\frac{i}{P \cdot\left(q+q^{\prime}\right)} \zeta^{\mu \nu \rho \sigma} P_{\rho}\left(q+q^{\prime}\right)_{\sigma} \widetilde{\mathcal{F}}_{1} \\
& +\mathcal{D}^{\mu \rho} \mathcal{D}^{\sigma \nu} \mathcal{G}_{\rho \sigma}+\frac{\xi}{P \cdot\left(q+q^{\prime}\right)}\left(\mathcal{D}^{\mu \rho} P_{\rho} \mathcal{D}^{\sigma \nu}+\mathcal{D}^{\mu \sigma} p_{\sigma} \mathcal{D}^{\rho \nu}\right) \mathcal{F}_{3 \rho}^{\perp}+\frac{i}{P \cdot\left(q+q^{\prime}\right)} \zeta^{\mu \nu \rho \sigma} q_{\sigma} \widetilde{\mathcal{F}}_{3 \rho}^{\perp},
\end{aligned}
$$

where the projection operator and $\xi$ are given by: 


$$
\begin{gathered}
\zeta^{\mu \nu \rho \sigma}=\epsilon^{\alpha \beta \rho \sigma}\left(g_{\alpha}^{\mu}-\frac{P^{\mu} q_{\alpha}^{\prime}}{P \cdot q^{\prime}}\right)\left(g_{\beta}^{\nu}-\frac{P^{\nu} q_{\beta}}{P \cdot q}\right), \\
\mathcal{D}^{\mu \nu}=g^{\mu \nu}-\frac{q^{\mu} q^{\prime \nu}}{q \cdot q^{\prime}} \quad \xi=x_{B} \frac{1+\frac{\Delta^{2}}{2 \mathcal{Q}^{2}}}{2-x_{B}+x_{B} \frac{\Delta^{2}}{\mathcal{Q}^{2}}} .
\end{gathered}
$$

By analogy with the terminology used in studies of deeply inelastic scattering, the functions that appear in the decomposition (1.41) will be called the Compton form factors (CFFs). Four of these functions, $\mathcal{F}_{1}, \mathcal{F}_{2}, \widetilde{\mathcal{F}}_{1}$ and $\mathcal{G}$ receive twist-two contributions at their leading term, while the other two, $\mathcal{F}_{3 \rho}^{\perp}$ and $\widetilde{\mathcal{F}}_{3 \rho}^{\perp}$, start at twist three. The appearance of the function $\mathcal{G}$ in (1.41) is a consequence of the gluon helicity-flip by two units. It arises due to nonzero orbital angular momentum in the off-forward scattering. The twist-two photon helicity-flip amplitude is absent in the handbag diagram due to the conservation of the angular momentum along the photon-parton collision axis. Since photons are vector particles, to flip their helicity one needs to compensate two units of the angular momentum. For the collinear twist-two partonic amplitude, this is only possible by a simultaneous flip of gluon helicities. Since quarks have spin one-half, their helicity flip can provide at most one unit of the angular momentum. At leading order, and therefore not taking into account twist-two photon helicity-flip or twist-three contributions, we are only interested in the CFFs $\mathcal{F}_{1}, \mathcal{F}_{2}$ and $\widetilde{\mathcal{F}}_{1}$.

$$
\begin{aligned}
& \mathcal{F}_{1}=\frac{1}{P^{+}} \bar{u}\left(p^{\prime}\right)\left(\gamma^{+} \mathcal{H}^{q}+\left(\gamma^{+}-\frac{\left(p+p^{\prime}\right)^{+}}{2 M_{p}}\right) \mathcal{E}^{q}\right) u(p), \\
& \widetilde{\mathcal{F}}_{1}=\frac{1}{P^{+}} \bar{u}\left(p^{\prime}\right)\left(\gamma^{+} \gamma^{5} \widetilde{\mathcal{H}}^{q}+\frac{\left(p^{\prime}-p\right)^{+}}{2 M_{p}} \gamma^{5} \widetilde{\mathcal{E}}^{q}\right) u(p) .
\end{aligned}
$$

The CFFs $\mathcal{F}_{1}$ and $\widetilde{\mathcal{F}}_{1}$ are linear combinations of the functions $\{\mathcal{H}, \mathcal{E}, \widetilde{\mathcal{H}}, \widetilde{\mathcal{E}}\}$, which we will label as CFFs as well. In general, these functions are given by a convolution of perturbatively calculable coefficient functions and a set of twist-two and twist-three GPDs. We now redefine our hadronic tensor taking into account that at leading order $\mathcal{F}_{2}=\xi \mathcal{F}_{1}$. The contribution to the cross section from DVCS has the form:

$$
\left|\left(2 M_{p} 2 m_{e}\right) \mathcal{T}_{D V C S}\right|^{2}=\sum_{S} \frac{\left(2 M_{p} 2 m_{e}\right)^{2} e^{6}}{q_{1}^{4}}\left(-g_{\mu \mu^{\prime}}\right) L_{\nu}^{D V C S} L_{\nu^{\prime}}^{\dagger D V C S} T^{\mu \nu} T^{\dagger \mu^{\prime} \nu^{\prime}}
$$

with 


$$
\begin{aligned}
& \sum_{S} L_{\nu}^{D V C S} L_{\nu^{\prime}}^{\dagger D V C S}=\frac{1}{4} \operatorname{Tr}\left[\left(\frac{\not k^{\prime}+m_{e}}{2 m_{e}}\right) \gamma_{\nu}\left(\frac{\not k+m_{e}}{2 m_{e}}\right) \gamma_{\nu^{\prime}}\right]=\left(\left(-g_{\nu \nu^{\prime}}\right) k \cdot k^{\prime}+k_{\nu} k_{\nu^{\prime}}^{\prime}+k_{\nu^{\prime}} k_{\nu}^{\prime}\right), \\
& \sum_{S} T^{\mu \nu} T^{\dagger \mu^{\prime} \nu^{\prime}}=\sum_{S}\left(D_{1}^{\mu \nu} D_{1}^{* \mu^{\prime} \nu^{\prime}} \mathcal{F}_{1} \mathcal{F}_{1}^{\dagger}+D_{1}^{\mu \nu} D_{2}^{* \mu^{\prime} \nu^{\prime}} \mathcal{F}_{1} \widetilde{\mathcal{F}}_{1}^{\dagger}+D_{2}^{\mu \nu} D_{1}^{* \mu^{\prime} \nu^{\prime}} \widetilde{\mathcal{F}}_{1} \mathcal{F}_{1}^{\dagger}+D_{2}^{\mu \nu} D_{2}^{* \mu^{\prime} \nu^{\prime}} \widetilde{\mathcal{F}}_{1} \widetilde{\mathcal{F}}_{1}^{\dagger}\right),
\end{aligned}
$$

where $D_{1}^{\mu \nu}$ and $D_{2}^{\mu \nu}$ are the combinations of the propagators defined in (1.42).

$$
\begin{aligned}
& D_{1}^{\mu \nu}=-\mathcal{D}^{\mu \rho} g_{\rho \sigma} \mathcal{D}^{\sigma \nu}+\frac{\xi}{P \cdot\left(q+q^{\prime}\right)} \mathcal{D}^{\mu \rho} P_{\rho} P_{\sigma} \mathcal{D}^{\sigma \nu}, \\
& D_{2}^{\mu \nu}=\frac{i}{P \cdot\left(q+q^{\prime}\right)} \zeta^{\mu \nu \rho \sigma} P_{\rho}\left(q+q^{\prime}\right)_{\sigma} .
\end{aligned}
$$

To find out the relation of the cross section with the CFFs $\{\mathcal{H}, \mathcal{E}, \widetilde{\mathcal{H}}, \widetilde{\mathcal{E}}\}$ we use the equations from (1.43) to resolve the combinations of $\mathcal{F}_{1}$ and $\widetilde{\mathcal{F}}_{1}$ that appear in the hadronic tensor shown in (1.45). Only two of these combinations have non-zero results:

$$
\begin{gathered}
\sum_{S} \mathcal{F}_{1} \mathcal{F}_{1}^{\dagger}=\frac{1}{\left(2 M_{p} p^{+}\right)^{2}}\left\{8 p^{+} p^{+} \mathcal{H} \mathcal{H}^{*}-2\left(\Delta^{+}\right)^{2}\left(\mathcal{E} \mathcal{H}^{*}+\mathcal{E}^{*} \mathcal{H}+\mathcal{E} \mathcal{E}^{*}\right)-\frac{\Delta^{2}}{2 M_{p}^{2}}\left(p^{+}\right)^{2} \mathcal{E} \mathcal{E}^{*}\right\} \\
\sum_{S} \widetilde{\mathcal{F}}_{1} \widetilde{\mathcal{F}}_{1}^{\dagger}=\frac{1}{\left(2 M_{p} p^{+}\right)^{2}}\left\{8 p^{+} p^{+} \widetilde{\mathcal{H}} \widetilde{\mathcal{H}}^{*}-2\left(\Delta^{+}\right)^{2}\left(\widetilde{\mathcal{E}} \widetilde{\mathcal{H}}^{*}+\widetilde{\mathcal{E}}^{*} \widetilde{\mathcal{H}}\right)-\frac{\Delta^{2}}{2 M_{p}^{2}}\left(\Delta^{+}\right)^{2} \widetilde{\mathcal{E}} \widetilde{\mathcal{E}}^{*}\right\} \\
\sum_{S} \mathcal{F}_{1} \widetilde{\mathcal{F}}_{1}^{\dagger}=0 \quad \sum_{S} \widetilde{\mathcal{F}}_{1} \mathcal{F}_{1}^{\dagger}=0 .
\end{gathered}
$$

We use both results found in (1.47) to define a variable which will enclose the dependence of the amplitude with the CFFs at twist-two level. By doing this, we will be able to express our cross section in terms of variables which contain the dependence on GPDs at different twist levels, and decompose it according to their contribution.

$$
\begin{aligned}
\sum_{S}\left(2 M_{p}\right)^{2} & \left(\mathcal{F}_{1} \mathcal{F}_{1}^{\dagger}+\widetilde{\mathcal{F}}_{1} \widetilde{\mathcal{F}}_{1}^{\dagger}\right)=\frac{2}{\left(2-x_{B}\right)^{2}}\left\{4\left(1-x_{B}\right)\left(\mathcal{H} \mathcal{H}^{*}+\widetilde{\mathcal{H}} \widetilde{\mathcal{H}}^{*}\right)\right. \\
& \left.-x_{B}^{2}\left(\mathcal{H} \mathcal{E}^{*}+\mathcal{E} \mathcal{H}^{*}+\widetilde{\mathcal{H}} \widetilde{\mathcal{E}}^{*}+\widetilde{\mathcal{E}} \widetilde{\mathcal{H}}^{*}\right)-\left(x_{B}^{2}+\left(2-x_{B}\right)^{2} \frac{\Delta^{2}}{4 M^{2}}\right) \mathcal{E} \mathcal{E}^{*}-x_{B}^{2} \frac{\Delta^{2}}{4 M^{2}} \widetilde{\mathcal{E}} \widetilde{\mathcal{E}}^{*}\right\} \\
& =2 \mathcal{C}_{u n p}^{D V C S}\left(\mathcal{F}, \mathcal{F}^{*}\right) .
\end{aligned}
$$


We observe that $\left|\mathcal{T}_{D V C S}\right|^{2}$ is bilinear in the CFFs, as opposed to the interference term, which we will study next. The subscript "unp" indicates our target is not polarized. At this point we can go back to equation (1.45) and combine the leptonic tensor with the $D_{1}^{\mu \nu}$ and $D_{2}^{\mu \nu}$ coefficients, left aside from the hadronic tensor computation, to find the $\phi$ dependence of the DVCS amplitude. Identifying each term, up to leading order, will give us the following:

$$
\begin{array}{r}
\sum_{S} \frac{\left(2 m_{e}\right)^{2} e^{6}}{q_{1}^{4}}\left(-g_{\mu \mu^{\prime}}\right) L_{\nu}^{D V C S} L_{\nu^{\prime}}^{\dagger D V C S} D_{1}^{\mu \nu} D_{1}^{* \mu^{\prime} \nu^{\prime}}=\sum_{S} \frac{\left(2 m_{e}\right)^{2} e^{6}}{q_{1}^{4}}\left(-g_{\mu \mu^{\prime}}\right) L_{\nu}^{D V C S} L_{\nu^{\prime}}^{\dagger D V C S} D_{2}^{\mu \nu} D_{2}^{* \mu^{\prime} \nu^{\prime}} \\
=-\frac{4 \mathcal{Q}^{2}}{y^{2}}\left(2-2 y+y^{2}\right)+\frac{16 \mathcal{Q}^{2} x_{B}(2-y)}{y^{2}\left(2-x_{B}\right)} K \cos (\phi)+\ldots
\end{array}
$$

where the dots stand for higher twist contributions. Combining equations (1.50) and (1.49) will give us the structure for the DVCS amplitude in terms of CFFs and $\phi$ we are looking for:

$$
\begin{aligned}
\left|\left(2 M_{p} 2 m_{e}\right) \mathcal{T}_{D V C S}\right|^{2}=\frac{e^{6}}{y^{2} \mathcal{Q}^{2}} & \left\{2\left(2-2 y+y^{2}\right) \mathcal{C}_{u n p}^{D V C S}\left(\mathcal{F}, \mathcal{F}^{*}\right)\right. \\
+ & \left.\frac{8 K}{2-x_{B}}(2-y) \Re e \mathcal{C}_{u n p}^{D V C S}\left(\mathcal{F}^{e f f}, \mathcal{F}^{*}\right) \cos (\phi)+\ldots\right\} .
\end{aligned}
$$

The $\phi$ independent term is known as the $c_{0}^{D V C S}$ coefficient, and it is expressed in terms of the twist-two CFFs $\{\mathcal{H}, \mathcal{E}, \widetilde{\mathcal{H}}, \widetilde{\mathcal{E}}\}$, while the $\cos (\phi)$ term, $c_{1}^{D V C S}$, has a twist-three component in its general expression, enclosed in the "effective" CFF, $\mathcal{F}^{e f f}$. In our computation we used that at leading order $\mathcal{F}^{e f f}=-x_{B} \mathcal{F}$ and didn't include the twist-three component in the result (see equation in Appendix C (C.16)). It can be shown that (1.51) leads to the more general expression [16]:

$$
\left|\left(2 M_{p} 2 m_{e}\right) \mathcal{T}_{D V C S}\right|^{2}=\frac{e^{6}}{y^{2} \mathcal{Q}^{2}}\left\{c_{0}^{D V C S}+\sum_{n=1}^{2} c_{n}^{D V C S} \cos (n \phi)+s_{n}^{D V C S} \sin (n \phi)\right\}
$$

where $s_{1}^{D V C S}$ arises from the interference of twist-two CFFs with $\mathcal{F}^{\text {eff }}$. The Fourier coefficients $c_{2}^{D V C S}$ and $s_{2}^{D V C S}$, come from gluon transversity. Again, one must include helicity operators to access $s_{1}^{D V C S}$ and $s_{2}^{D V C S}$. The results for the Fourier coefficients, presented above, only include contributions up to the twist-three level. 


\section{Interference term}

For the phenomenology of GPDs, the interference term is the most interesting quantity since it is linear in CFFs and thus simplifies their extraction from experimental measurements. It is defined as the combination of the DVCS and BH amplitudes as follows:

$$
\mathcal{I}=\left(2 M_{p} 2 m_{e}\right)^{2} 2 \Re e\left[\mathcal{T}_{D V C S} \mathcal{T}_{B H}^{*}\right]=\sum_{S} \frac{\left(2 M_{p} 2 m_{e}\right)^{2} e^{6}}{\Delta^{2} q_{1}^{2}}\left(-g_{\mu \mu^{\prime}}\right) 2 L_{\nu}^{D V C S} L_{\mu^{\prime} \nu^{\prime}}^{\dagger B H} T^{\mu \nu} J^{\nu^{\prime}}
$$

with

$$
\begin{aligned}
& \sum_{S} L_{\nu}^{D V C S} L_{\mu^{\prime} \nu^{\prime}}^{\dagger B H}=\frac{1}{4} \operatorname{Tr}\left[\left(\frac{\not k^{\prime}+m_{e}}{2 m_{e}}\right) \gamma_{\nu}\left(\frac{\not k+m_{e}}{2 m_{e}}\right)\left(\gamma_{\nu^{\prime}} \frac{1}{\not k-\not \Delta} \gamma_{\mu^{\prime}}+\gamma_{\mu^{\prime}} \frac{1}{\not k^{\prime}+\not} \gamma_{\nu^{\prime}}\right)\right], \\
& \sum_{S} T^{\mu \nu} J^{\nu^{\prime}}=\sum_{S}\left(D_{1}^{\mu \nu} \mathcal{F}_{1}+D_{2}^{\mu \nu} \widetilde{\mathcal{F}}_{1}\right) J^{\nu^{\prime}} .
\end{aligned}
$$

Once more we take only into account the contribution at leading order from the CFFs $\mathcal{F}_{1}, \mathcal{F}_{2}$ and $\widetilde{\mathcal{F}}_{1} . D_{1}^{\mu \nu}$ and $D_{2}^{\mu \nu}$ are the very same combinations of propagators defined for the DVCS amplitude. In order to simplify the computation, we split the leptonic tensor into two terms, $L_{\nu \mu \nu^{\prime}}^{\mathcal{I} s}$ and $L_{\nu \mu \nu^{\prime}}^{\mathcal{I} a}$, symmetric and antisymmetric leptonic currents of the interference amplitude. We define them by the relations:

$$
\begin{aligned}
& L_{\nu \mu \nu^{\prime}}^{\mathcal{I} s}=\operatorname{Tr}\left[\not k^{\prime} \gamma_{\nu} \not k\left(\gamma_{\nu^{\prime}}(\not k-\Delta) \gamma_{\mu}+\gamma_{\mu}\left(\not k^{\prime}+\Delta\right) \gamma_{\nu^{\prime}}\right)\right], \\
& L_{\nu \mu \nu^{\prime}}^{\mathcal{I} a}=\operatorname{Tr}\left[\not k^{\prime} \gamma_{\nu} \not k\left(\gamma_{\nu^{\prime}}(\not k-\Delta) \gamma_{\mu}-\gamma_{\mu}\left(\not k^{\prime \prime}+\Delta\right) \gamma_{\nu^{\prime}}\right)\right] .
\end{aligned}
$$

Combining these expressions with the hadronic tensor will yield two contributions to the interference term: one related to $\mathcal{F}_{1}$ and the other to $\widetilde{\mathcal{F}}_{1}$. Both contain a $1 / \mathcal{P}_{1}(\phi) \mathcal{P}_{2}(\phi)$ factor that will remain in the global factor of the harmonic decomposition of the interference amplitude.

$$
\begin{array}{r}
\mathcal{I}=\sum_{S} \frac{\left(2 M_{p}\right)^{2} e^{6}}{\Delta^{2} \mathcal{Q}^{2}} \frac{1}{\mathcal{Q}^{4} \mathcal{P}_{1}(\varphi) \mathcal{P}_{2}(\varphi)}\left\{D_{1}^{\mu \nu} \mathcal{F}_{1} J^{\nu^{\prime}}\left(L_{\nu \mu \nu^{\prime}}^{\mathcal{I}_{s}}\left(\mathcal{Q}^{2}+\Delta^{2}\right)+L_{\nu \mu \nu^{\prime}}^{\mathcal{I} a}\left(\left(\mathcal{Q}^{2}-\Delta^{2}\right)+4 k \cdot \Delta\right)\right)\right. \\
\left.+D_{2}^{\mu \nu} \widetilde{\mathcal{F}}_{1} J^{\nu^{\prime}}\left(L_{\nu \mu \nu^{\prime}}^{\mathcal{I} s}\left(\mathcal{Q}^{2}+\Delta^{2}\right)+L_{\nu \mu \nu^{\prime}}^{\mathcal{I} a}\left(\left(\mathcal{Q}^{2}-\Delta^{2}\right)+4 k \cdot \Delta\right)\right)\right\},
\end{array}
$$

where the following relations for the combinations of $\mathrm{BH}$ propagators were used: 


$$
\frac{1}{(k-\Delta)^{2}}+\frac{1}{\left(k^{\prime}+\Delta\right)^{2}}=\frac{\mathcal{Q}^{2}+\Delta^{2}}{\mathcal{Q}^{4} \mathcal{P}_{1}(\phi) \mathcal{P}_{2}(\phi)} \quad \frac{1}{(k-\Delta)^{2}}-\frac{1}{\left(k^{\prime}+\Delta\right)^{2}}=\frac{\left(\mathcal{Q}^{2}-\Delta^{2}\right)+4 k \cdot \Delta}{\mathcal{Q}^{4} \mathcal{P}_{1}(\phi) \mathcal{P}_{2}(\phi)}
$$

Additionally, both terms in (1.56) can be independently decomposed into Fourier harmonics, with a dependence on $\cos (\phi), \cos ^{2}(\phi)$ and a $\phi$ independent term. That is, if we stop at leading order and don't include helicity operators:

$$
\begin{array}{r}
\mathcal{I}=\frac{e^{6}}{x_{B} y^{3} \Delta^{2} \mathcal{P}_{1}(\phi) \mathcal{P}_{2}(\phi)}\left\{c_{10}^{\mathcal{I}}+c_{11}^{\mathcal{I}} \cos (\phi)+c_{12}^{\mathcal{I}} \cos ^{2}(\phi)+\ldots\right. \\
\left.c_{20}^{\mathcal{I}}+c_{21}^{\mathcal{I}} \cos (\phi)+c_{22}^{\mathcal{I}} \cos ^{2}(\phi)+\ldots\right\} .
\end{array}
$$

In the last equation we identify the term $c_{10}^{\mathcal{I}}$ as the $\phi$ independent term resulting from the $D_{1}^{\mu \nu} \mathcal{F}_{1}$ contribution. The term $c_{20}^{\mathcal{I}}$ results from the $D_{2}^{\mu \nu} \widetilde{\mathcal{F}}_{1}$ contribution. In a more general way the interference amplitude has the expression as [16],

$$
\mathcal{I}=\frac{ \pm e^{6}}{x_{B} y^{3} \Delta^{2} \mathcal{P}_{1}(\phi) \mathcal{P}_{2}(\phi)}\left\{c_{0}^{\mathcal{I}}+\sum_{n=1}^{3} c_{n}^{\mathcal{I}} \cos (n \phi)+s_{n}^{\mathcal{I}} \sin (n \phi)\right\}
$$

Here, the $+(-)$ sign stands for the negatively (positively) charged lepton beam. It is very important to notice that the DVCS amplitude changes its sign when one goes from the electron to the positron beam, $\left.\mathcal{T}_{D V C S}\right|_{e+}=-\left.\mathcal{T}_{D V C S}\right|_{e-}$ while the BH process does not, $\left.\mathcal{T}_{B H}\right|_{e+}=\left.\mathcal{T}_{B H}\right|_{e-}$. The coefficients $c_{0}^{\mathcal{I}}, c_{1}^{\mathcal{I}}$ and $s_{1}^{\mathcal{I}}$ arise at twist-two level, while $c_{2}^{\mathcal{I}}$ and $s_{2}^{\mathcal{I}}$ depend on the effective twist-three CFFs. Coefficients $c_{3}^{\mathcal{I}}$ and $s_{3}^{\mathcal{I}}$ originate from the twist-two double helicity-flip gluonic GPDs alone (the $s_{i}^{\mathcal{I}}$ coefficients also depend on the electron helicity).

\subsection{Experiment goals}

As we have seen, the photon electroproduction cross section of a polarized lepton beam of energy $E_{b}$ off an unpolarized target is sensitive to the coherent interference of the DVCS amplitude with the Bethe-Heitler amplitude. In summary, the photon electroproduction helicity-independent $(\mathrm{d} \sigma)$ and helicity-dependent $(\mathrm{d} \Sigma)$ cross sections read [10]:

$$
\begin{aligned}
\frac{d^{5} \sigma}{d^{5} \Phi}=\frac{1}{2}\left[\frac{d^{5} \sigma^{+}}{d^{5} \Phi}+\frac{d^{5} \sigma^{-}}{d^{5} \Phi}\right] & =\Gamma^{G}\left|\mathcal{T}_{B H}\right|^{2}+\Gamma_{0}^{D V C S} \mathcal{C}^{D V C S}\left(\mathcal{F}, \mathcal{F}^{*}\right)+\left(\Gamma_{0}^{\mathcal{I}}-\Gamma_{1}^{\mathcal{I}} \cos (\phi)\right) \Re e\left[\mathcal{C}^{\mathcal{I}}(\mathcal{F})\right] \\
& +\Gamma_{0, \Delta}^{\mathcal{I}} \Re e\left[\mathcal{C}^{\mathcal{I}}+\Delta \mathcal{C}^{\mathcal{I}}\right](\mathcal{F})+\cos (2 \phi) \Gamma_{2}^{\mathcal{I}} \Re e\left[\mathcal{C}^{\mathcal{I}}\left(\mathcal{F}^{e f f}\right)\right],
\end{aligned}
$$




$$
\begin{aligned}
& \frac{d^{5} \Sigma}{d^{5} \Phi}=\frac{1}{2}\left[\frac{d^{5} \sigma^{+}}{d^{5} \Phi}-\frac{d^{5} \sigma^{-}}{d^{5} \Phi}\right]=\sin (\phi) \Gamma_{1}^{\mathcal{I}} \Im m\left[\mathcal{C}^{D V C S}\left(\mathcal{F}^{e f f}, \mathcal{F}^{*}\right)\right] \\
& +\sin (\phi) \Gamma_{1}^{\mathcal{I}} \Im m\left[\mathcal{C}^{\mathcal{I}}(\mathcal{F})\right]-\sin (2 \phi) \Gamma_{2}^{\mathcal{I}} \Im m\left[\mathcal{C}^{\mathcal{I}}\left(\mathcal{F}^{e f f}\right)\right],
\end{aligned}
$$

where $d^{5} \Phi=d x_{B} d y d\left|\Delta^{2}\right| d \phi d \varphi$. The $\mathcal{C}^{D V C S}$ and $\mathcal{C}^{\mathcal{I}}$ terms are the combinations of CFFs, from DVCS ${ }^{2}$ and interference contributions (Appendix C), we will relate the photon electroproduction cross section to. We only kept the leading (twist-2) contribution $\mathcal{C}^{D V C S}\left(\mathcal{F}, \mathcal{F}^{*}\right)$ to the $\operatorname{DVCS}^{2}$ term in the helicity-independent cross section. Only a twist3 DVCS $^{2}$ contribution is present in the helicity-dependent cross section. The mixing of the DVCS $^{2}$ term with the twist- $3 \sin (2 \phi)$ and $\cos (2 \phi)$ observables has been also suppressed. In the case of the helicity-independent cross section we take the real part of all these terms while we use the imaginary part in the helicity-dependent case. The $\Gamma_{i}$ are kinematic factors which depend on the beam energy. The extremely rich harmonic structure of the DVCS cross section as a function of the azimuthal angle $\phi$ provides an excellent tool to separate its different contributions. However, even though the DVCS ${ }^{2}$ terms in the cross section have a different $\phi$ dependence than the interference terms (due to the absence of the $\mathrm{BH}$ propagators), the dependence is not sufficiently different to allow, in the present state, a reliable separation of these terms. Nevertheless, the angular separation can be supplemented by an energy separation.

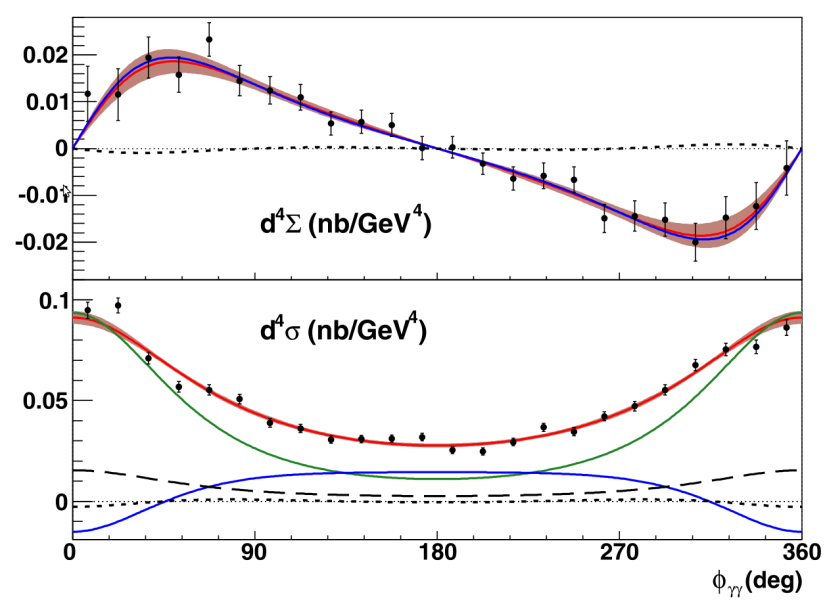

Figure 1.15: (Red) DVCS helicity-dependent $\left(d^{4} \Sigma\right)$ and helicity-independent $\left(d^{4} \sigma\right)$ cross sections, measured in E00-110 for $\mathcal{Q}^{2}=2.3 \mathrm{GeV}^{2}$ and $t=0.28 \mathrm{GeV}^{2}$. (Green) BH. (Blue) $\mathcal{C}^{\mathcal{I}}(\mathcal{F})$ (including $\mathcal{C}^{D V C S}\left(\mathcal{F}^{\text {eff }}, \mathcal{F}^{*}\right)$ ) contribution. (Dashed line) $\left[\mathcal{C}^{\mathcal{I}}+\Delta \mathcal{C}^{\mathcal{I}}\right](\mathcal{F})$ contribution. (Dotted line) $\mathcal{C}^{\mathcal{I}}\left(\mathcal{F}^{e f f}\right)$ contribution.

Experiment E07-007 is focused on separating the interference terms $\mathcal{C}^{\mathcal{I}}(\mathcal{F})$ and $\left[\mathcal{C}^{\mathcal{I}}+\Delta \mathcal{C}^{\mathcal{I}}\right](\mathcal{F})$ from the $\operatorname{DVCS}^{2}$ term $\mathcal{C}^{D V C S}\left(\mathcal{F}, \mathcal{F}^{*}\right)$ in the helicity-independent cross section. Experimentally, we can determine $\mathcal{C}^{D V C S}\left(\mathcal{F}, \mathcal{F}^{*}\right)$ if we make an accurate cross section measurement 
at constant $x_{B}$ and $t$ (and $\mathcal{Q}^{2}$ ) at two different incident beam energies (hence the name of Rosenbluth Separation), since only the kinematic factors $\Gamma_{i}$ are affected by this change whereas the extracted coefficients do not. A measurement of $\mathcal{C}^{D V C S}\left(\mathcal{F}, \mathcal{F}^{*}\right)$ is essential in order to properly interpret the experimental DVCS observables in terms of GPDs. A similar experiment to E07-007, measuring the cross section for deeply virtual Compton scattering off the deuteron (JLab E08-025), is being carried on [36]. There are several indications that the size of the $\operatorname{DVCS}^{2}$ term, $\mathcal{C}^{D V C S}\left(\mathcal{F}, \mathcal{F}^{*}\right)$, can be important. Some of these indications come from the high precision data obtained in a previous experiment, E00-110 at Jefferson Lab. Figure 1.15 shows (for one bin in $\mathcal{Q}^{2}$ and $t$ ) the DVCS cross sections measured in E00-110 [37]. The green curve in the unpolarized cross section $d^{4} \sigma$ (lower panel) shows the contribution of the BH to the cross section. The BH only accounts for half of the total cross section between $90^{\circ}$ and $270^{\circ}$. It is unlikely that the $\mathrm{DVCS}^{2}$ contribution is negligible, since the interference term (BH.DVCS) alone could not possibly account for the difference in this case. Theoretical models also predict this contribution to be large [38], [39], [40]. In order to estimate the accuracy on the extraction of the DVCS ${ }^{2}$ (and the interference) terms, a simulation of the extraction procedure was performed. In this new experiment, we have an additional $\Gamma_{0}^{D V C S}$, associated with $\mathcal{C}^{D V C S}\left(\mathcal{F}, \mathcal{F}^{*}\right)$. We can see its contribution to the cross section as the magenta line of the Figure 1.16, which shows some results of the simulation:
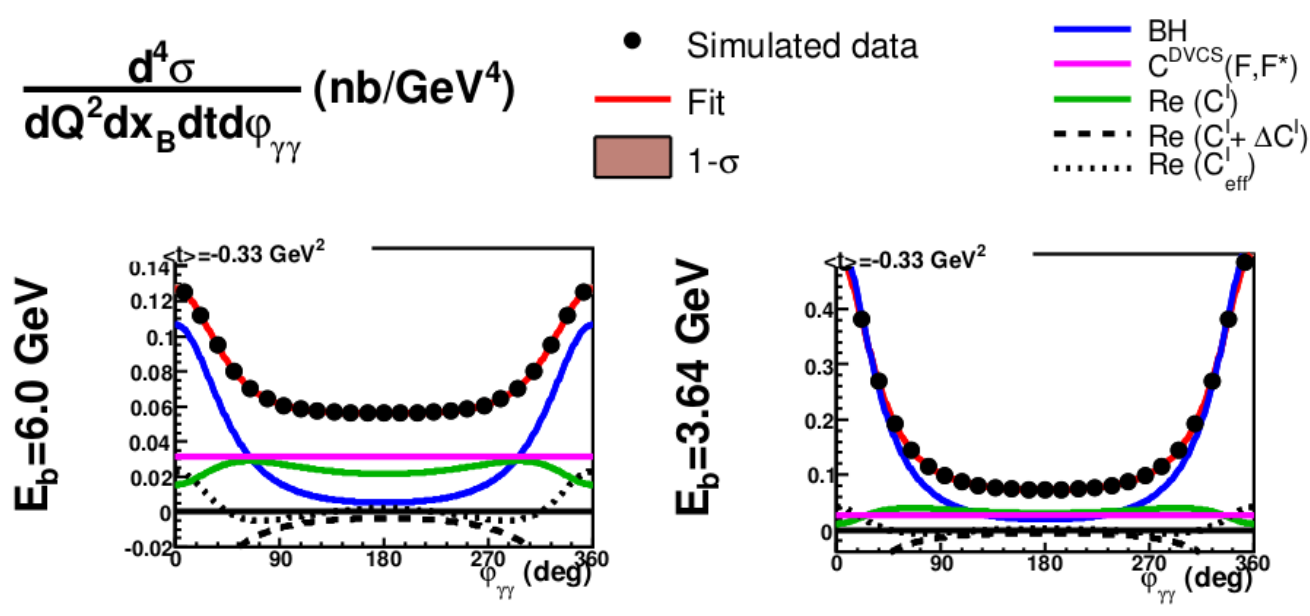

Figure 1.16: Simulated cross-section measurements for the $\mathcal{Q}^{2}=1.5 \mathrm{GeV}^{2}$ setting at two different beam energies. $\mathcal{C}^{D V C S}\left(\mathcal{F}, \mathcal{F}^{*}\right)=20$. Note that the differential cross section only depends on 4 variables after the integration over $\varphi$, since this variable contains no physics. The dependence on the kinematic variable $y$ has been replaced by the dependence on $\mathcal{Q}^{2}$, through the expression $\mathcal{Q}=E_{b} \epsilon y$. 
In summary, the experiment is expected to perform a meaningful separation of the DVCS $^{2}$, or, if this contribution is small, to draw a decisive conclusion about its size. Below, a table with the different settings for every kinematic is shown, $\left(q^{\prime}\right.$ stands for the virtual photon energy):

\begin{tabular}{|c|c|c|c|}
\hline & Kinematics 1 & Kinematics 2 & Kinematics 3 \\
\hline $\mathcal{Q}^{2}\left(G e V^{2}\right)$ & 1.5 & 1.75 & 2 \\
\hline$x_{B}$ & 0.36 & 0.36 & 0.36 \\
\hline$W^{2}\left(G e V^{2}\right)$ & 3.55 & 3.99 & 4.44 \\
\hline$q^{\prime}(G e V)$ & 2.14 & 2.51 & 2.88 \\
\hline$E_{b}(G e V)$ & \begin{tabular}{l|l}
5.552 & 3.356
\end{tabular} & \begin{tabular}{l|l|}
5.552 & 4.454
\end{tabular} & \begin{tabular}{l|l|}
5.552 & 4.454
\end{tabular} \\
\hline
\end{tabular}

Table 1.2: Different settings for the kinematics employed in experiment E07-007. $q^{\prime}=k-k^{\prime}$.

We must take into account that in order to maintain the same net statistical contribution to the DVCS separations from the two beam energy settings, one must increase the beam time at the lower energy setting. 



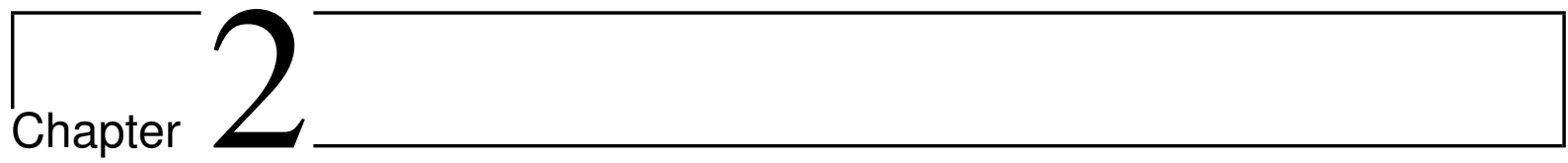

\section{Experimental setup}

Experiment E07-007, "Rosenbluth separation of DVCS cross section", on which this thesis is based, was conducted in Hall A at Jefferson Lab during the period comprehended between October and December of 2010. In this chapter a detailed description of the main instrumentation used in the experimental setup will be given. We begin by a general description of the laboratory and more precisely, of Hall A.

\section{$2.1 \quad$ Jefferson Lab}

Located in Newport News, Virginia (U.S.A.), the laboratory's main research facility is the Continuous Electron Beam Accelerator Facility (CEBAF) [41]. Its scientific goal is to investigate the structure of nuclei and hadrons and the underlying fundamental interactions in the region below the high-energy "asymptotically free" regime. CEBAF consists of a polarized electron source and injector and a pair of superconducting RF linear accelerators, which operate with sinusoidally varying electromagnetic fields at radio frequency, of $300 \mathrm{~m}$ in length, connected to each other by two arc sections which contain steering magnets. Each linac contains 20 cryomodules with a design accelerating gradient of $5 \mathrm{MeV} / \mathrm{m}$. Ongoing in situ processing resulted in an average gradient in excess of 7 $\mathrm{MeV} / \mathrm{m}$, which made it possible to accelerate electrons to a maximum of $6 \mathrm{GeV}$ as the beam makes up to five successive orbits.

The design of CEBAF allows the electron beam to be continuous rather than the pulsed beam typical of ring shaped accelerators. There is some beam structure but the pulses are very much shorter and closer together. One bunch can be peeled off after each linac pass to any one of the Halls, labelled Hall A, Hall B, and Hall C, using RF separators and septa, partitions that separate two field regions. Each one of these facilities can receive simultaneously maximum beam energy and is equipped with the necessary instruments to record the results of collision between the electron beam and a stationary 
target (Figure 2.1). A fourth experimental hall, known as Hall D [42], is currently under construction and is scheduled to begin experiments in 2015. The new experimental Hall $\mathrm{D}$ will use the electron beam to produce a coherent bremsstrahlung beam and house a solenoid detector to carry out a program in gluonic spectroscopy to experimentally test current understanding of quark confinement.

Currently, Jefferson Lab is undergoing an upgrading process after which the electron beam is expected to reach $12 \mathrm{GeV}$ [43], [44]. Figure 2.2 shows the main modifications being carried on.

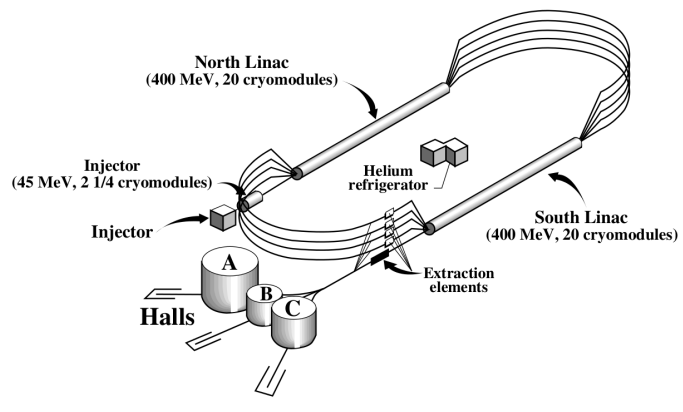

Figure 2.1: CEBAF and Halls A, B and C.

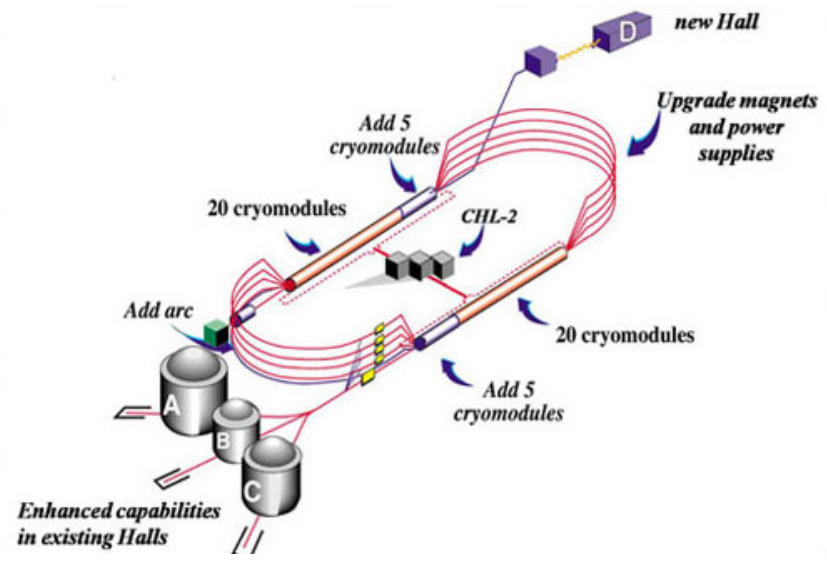

Figure 2.2: Schematic layout of the required facility modifications to realize the $12 \mathrm{GeV}$ Upgrade.

\subsection{Hall A}

Hall A is used for experiments which require high luminosity and/or high resolution in momentum and/or angle for at least one of the reaction products. The hall is used primarily for experiments that study the structure of the nucleus and the protons and neutrons it contains, focusing on nucleon form factors to high $\mathcal{Q}^{2}$, the strange-quark structure of the proton, nucleon spin structure, few-body form factors to high $\mathcal{Q}^{2}$ and nuclear structure at small inter-nucleon separations [45].

The basic layout of Hall A is shown in figure 2.3. The central elements are the two High Resolution Spectrometers (HRS). Both of these devices provide a momentum resolution better than $2 \cdot 10^{-4}$ and a horizontal angular resolution of better than 2 mrad at a design maximum central momentum of $4 \mathrm{GeV} / \mathrm{c}$. The spectrometers must have high resolution 
to be able to isolate the different reaction channels in nuclei so that a clean comparison with theory can be achieved.

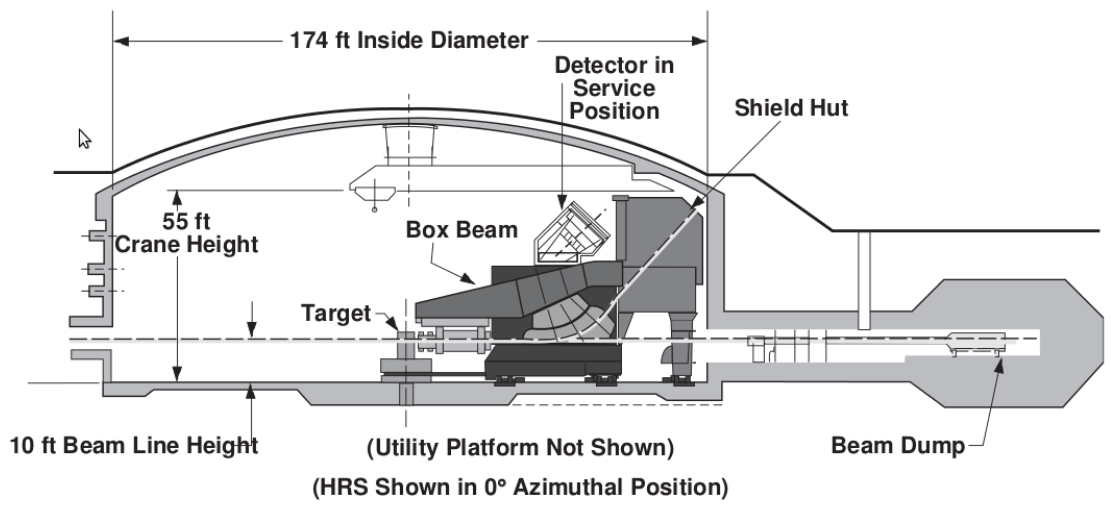

Figure 2.3: Hall A schematic layout.

The spectrometers are known as "Left" and "Right" (HRS-L and HRS-R). During the DVCS experiment only one of the spectrometers was used, HRS-L. We move on to the description of this device as well as the components along the beamline [46], the target chamber and the electromagnetic calorimeter that was installed for the experiment purpose.

\subsubsection{High Resolution Spectrometer (HRS)}

During the experiment the HRS-L was used to detect the scattered electron in DVCS reactions. Its basic layout is shown in figure 2.4. The vertically bending design includes a pair of superconducting $\cos (2 \theta)$ quadrupoles followed by a $6.6 \mathrm{~m}$ long dipole magnet with focussing entrance and exit polefaces and including additional focussing from a field gradient in the dipole. Following the dipole is a third superconducting $\cos (2 \theta)$ quadrupole.

Both spectrometers were designed to be able to identify the different charged particles going through them. Their main functions include: providing a trigger to activate the data-acquisition electronics, collecting tracking information (position and direction), precise timing for time-of-flight measurements and coincidence determination, and identification of the scattered particles. The timing information is provided from scintillators, as well as the main trigger. The particle identification is obtained from a variety of Čherenkov type detectors (aerogel and gas) and lead-glass shower counters[47]. A pair of Vertical Drift Chambers (VDCs) provide tracking information [48]. The main part of the detector package in the two spectrometers (trigger scintillators and VDCs) is identical, 
but the arrangement of particle-identification detectors differs slightly.

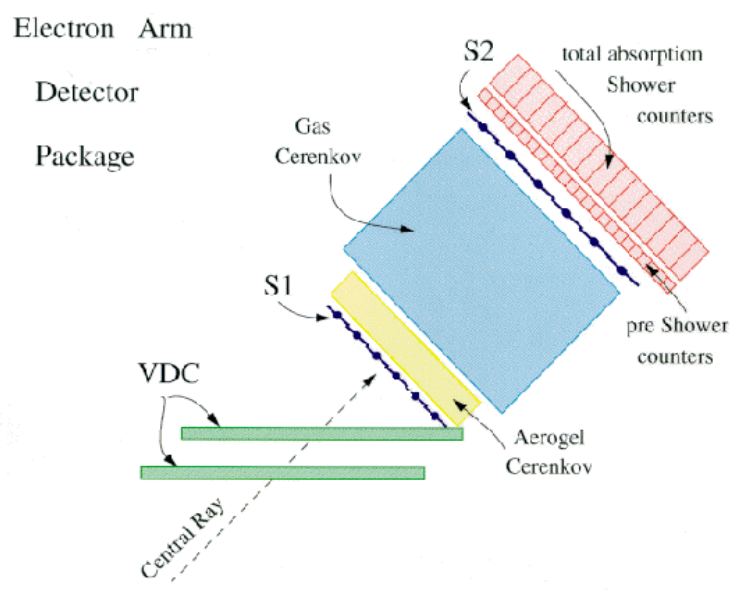

Figure 2.4: Schematic layout of the detector package of the HRS-L.

Tracking information is provided by a pair of VDCs in each HRS. The concept of VDCs fits well into the scheme of a spectrometer with a small acceptance, allowing a simple analysis algorithm and high efficiency, because multiple tracks are rare. Each VDC chamber is composed of two wire planes, separated by about $335 \mathrm{~mm}$, in a standard UV configuration (the wires of each successive plane are oriented at $90^{\circ}$ to one another, and lie in the laboratory horizontal plane).

There are two primary trigger scintillator planes (S1 and S2), separated by a distance of about $2 \mathrm{~m}$. Each plane is composed of six overlapping paddles made of thin plastic scintillator to minimize hadron absorption. Each scintillator paddle is viewed by two photomultipliers (PMTs). The time resolution per plane is approximately 0.30ns. For experiments which need a high hadron trigger efficiency, an additional scintillator trigger counter ( $\mathrm{S} 0$ ) can be installed. A gas Čherenkov detector filled with $\mathrm{CO}_{2}$ at atmospheric pressure is mounted between the trigger scintillator planes $\mathrm{S} 1$ and $\mathrm{S} 2$. The detector allows an electron identification with $99 \%$ efficiency and has a threshold for pions at $4.8 \mathrm{GeV} / \mathrm{c}$.

Each spectrometer is equipped with two layers of shower detectors. The blocks in both shower detectors in HRS-L and in the first layer in HRS-R are oriented perpendicular to the particle tracks. In the second layer of HRS-R, the blocks are parallel to the tracks. The dimensions of the rear straw chambers were chosen to be large enough that the geometrical efficiency is nearly $100 \%$ for a scattering angle up to $20^{\circ}$ for the full acceptance of the HRS. 


\subsection{Beamline}

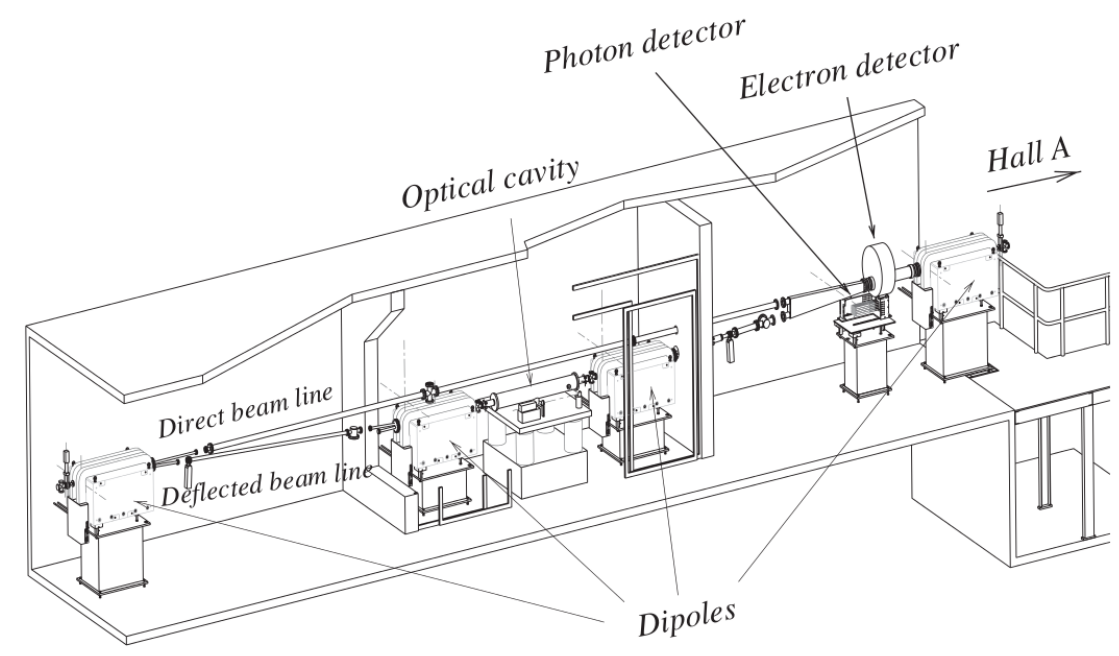

Figure 2.5: Schematic layout of the Compton polarimeter, showing the four dipoles of the chicane, the optical cavity and the photon and electron detector.

The instrumentation along the beamline [49] consists of various elements necessary to transport the electron beam onto the target and into the dump, and to measure simultaneously the relevant properties of the beam. Such instrumentation allows us to determine the beam energy, its current and polarization, and also the position, direction, size and stability of the beam at the Hall A target location. The beam diagnostic elements consist of transmission-line position monitors, current monitors, superharps, viewers, loss monitors and optical transition radiation (OTR) viewers.

\subsubsection{Beam position}

To determine the position and direction of the beam at the target location, two Beam Position Monitors (BPMs) are located upstream of the target. The standard differenceover-sum technique is then used to determine the relative position of the beam to within $100 \mu \mathrm{m}$ for currents above $1 \mu \mathrm{A}$ [50]. The absolute position of the beam can be determined from the BPMs by calibrating them with respect to wire scanners which are located adjacent to each of the BPMs. 


\subsubsection{Beam current}

In order to measure the beam current a Beam Current Monitor (BCM) was installed in Hall A. It is designed for a stable, low-noise, non-interfering beam current measurement. It consists of an Unser monitor, two RF cavities and the associated electronics. The cavities and the Unser monitor are enclosed in a temperature-stabilized box to improve magnetic shielding.

The Unser monitor is a Parametric Current Transformer which provides an absolute reference [51]. The monitor is calibrated by passing a known current through a wire inside the beam pipe and has a nominal output of $4 \mathrm{mV} / \mu \mathrm{A}$. The Unser monitor cannot be used to continuously monitor the beam current since its output signal drifts significantly on a time scale of several minutes. To minimize this drifting the Unser monitor is equipped with an extensive magnetic shielding and temperature stabilization to reduce noise. The two resonant RF cavity monitors on either side of the Unser Monitor are stainless steel cylindrical waveguides which are tuned to the frequency of the beam $(1.497 \mathrm{GHz})$ resulting in voltage levels at their outputs which are proportional to the beam current.

\subsubsection{Beam energy}

The energy of the beam is measured absolutely by the so called Arc method [52]. This method determines the energy by measuring the deflection of the beam in the arc section of the beamline. The measurement is made when the beam is tuned in dispersive mode in the arc section. The momentum of the beam $\left(p_{b}\right.$ in $\left.\mathrm{GeV} / \mathrm{c}\right)$ is then related to the field integral of the eight dipoles $\left(\int \vec{B} \cdot \overrightarrow{d l}\right.$ in T.m) and the net bend angle through the arc section ( $\theta$ in radians) by

$$
p_{b}=k_{b} \frac{\int \vec{B} \cdot \overrightarrow{d l}}{\theta}
$$

where $k_{b}=0.299792 \mathrm{GeV} \mathrm{rad} \mathrm{T}^{-1} \mathrm{~m}^{-1} / \mathrm{c}$. For the measurement to be successful one must simultaneously measure the magnetic field integral of the bending elements (eight dipoles in the arc), based on a reference magnet (9th dipole) measurement, and the actual bend angle of the arc, based on a set of wire scanners.

\subsubsection{Polarimeters}

One of the goals of the E07-007 experiment is to obtain helicity dependent cross sections of the photon electroproduction reaction. Therefore it is important to measure the polarization of the electron beam delivered to the hall A, with a typical beam polarization of $75-85 \%$. The beamline is equipped with two polarimeters, the Møller and the 
Compton polarimeters, whose functions are partly overlapping and partly complementary.

\section{Møller Polarimeter}

The purpose of the Møller polarimeter is to measure the longitudinal component of the beam polarization. To do so a ferromagnetic foil, magnetized in a magnetic field of about $24 \mathrm{mT}$ along its plane, is set as a target for the beam polarized electrons. The magnetized foil is placed in order to exploit the process of Møller scattering of polarized electrons off polarized atomic electrons. It is possible to calculate the beam polarization by studying the Møller scattering cross section, which depends on the beam and target polarizations.

The target foil can be tilted at various angles to the beam in the horizontal plane, providing a target polarization that has both longitudinal and transverse components. The spin of the incoming electron beam may have a transverse component due to precession in the accelerator and in the extraction arc. The asymmetry is measured at two target angles of about $\pm 20^{\circ}$ and the average is taken. Because the transverse contributions have opposite signs for these target angles, the transverse contributions cancel in the average.

A magnetic spectrometer consisting of a sequence of three quadrupole magnets and a dipole magnet detects the Møller scattering events. It is not possible to measure the beam polarization at the same time when the experiment is running due to the invasive method of the process. The measurements with the Møller typically take an hour, providing a statistical accuracy of about $0.2 \%$.

The dominant uncertainty of the Møller polarimeter comes from the uncertainty in the target polarization. Another uncertainty comes from the fact that the Møller polarimeter has to use a low beam current $(0.5 \mu \mathrm{A}$, typically). The current is reduced at the injector, either by attenuating the laser light or with a slit at the chopper, each of which might change the beam polarization.

\section{Compton Polarimeter}

The Compton polarimeter, utilizing the process of Compton scattering, analyzes the beam polarization from the measurement of the counting rate asymmetry for opposite beam helicities in the scattering of a circularly polarized photon beam by the electron beam [53]. Opposite to the Møller polarimeter (which measurements are invasive), it was designed to measure the beam polarization concurrently with experiments running in the hall to a $1 \%$ statistical error within an hour. Installed at the entrance of the hall, the Compton polarimeter consists of a magnetic chicane, a photon source, an electromagnetic calorimeter, and an electron detector as shown in figure 2.5. 
The electron beam is deflected vertically by the four dipoles of the chicane and crosses the photon beam at the Compton interaction point. After interaction, the backscattered photons are detected in the calorimeter and the electrons in the silicon strip electron detector located a few $\mathrm{mm}$ above the primary beam in front of the fourth dipole. Electrons that did not interact exit the polarimeter and reach the target.

\subsection{The target system}

For DVCS experiment on the proton a liquid hydrogen $\left(\mathrm{LH}_{2}\right)$ target was employed. The cryogenic target system in Hall A is mounted inside a scattering chamber along with subsystems for cooling, gas handling, temperature and pressure monitoring, target control and motion, and an attached calibration and solid target ladder. The operating temperature and pressure of the $\mathrm{LH}_{2}$ target are $19 \mathrm{~K}$ and $0.17 \mathrm{MPa}$, with a density of about $0.0723 \mathrm{~g} / \mathrm{cm}^{3}$. A liquid deuterium $\left(\mathrm{LD}_{2}\right)$ target was used for DVCS experiment on the neutron, with the following values for temperature and pressure: $22 \mathrm{~K}$ and $0.15 \mathrm{MPa}$, and a density of $0.167 \mathrm{~g} / \mathrm{cm}^{3}$.

Each of the two liquid loops has two aluminum cylindrical target cells mounted on a target ladder. The cells are $63.5 \mathrm{~mm}$ in diameter and $15 \mathrm{~cm}$ long. The sidewalls of the cells are $178 \mathrm{~mm}$ thick, with entrance and exit windows approximately 71 and $102 \mu \mathrm{m}$ thick, respectively. The target is in an enclosure filled with helium. Helium cooling jets on the target windows prevent the electron beam from overheating the target cell. Both liquid targets are sub-cooled by $3 \mathrm{~K}$. Along with these two targets, the basic cryogenic target system has a gaseous helium loop.

Besides the three mentioned targets, there are three dummy targets and a solid target ladder with five positions. The dummy targets, used to measure contributions from the windows, contain two thin pieces of aluminum. The positions of the solid target ladder are usually occupied by a $\mathrm{BeO}$ target, an empty target, a $12 \mathrm{C}$ target, used to study the spectrometer acceptance, and two special-purpose targets. The solid targets are usually not cooled.

\subsection{Electromagnetic Calorimeter}

The electromagnetic calorimeter is an upgraded version of the very same device used in previous DVCS experiments. It used to be composed of 132 lead fluoride $\left(\mathrm{PbF}_{2}\right)$ blocks arranged in 12 rows and 11 columns. The upgrade consisted on incrementing its size for a larger angle coverage. Now it is composed of $208 \mathrm{PbF}_{2}$ blocks disposed in 16 rows and 
13 columns. Lead fluoride has the following characteristics: a density of $7.77 \mathrm{~g} / \mathrm{cm}^{3}$, a radiation length of $0.93 \mathrm{~cm}$, a Molière radius of $2.2 \mathrm{~cm}$ and a refraction index of 1.8 . The surface of the blocks is $3 \times 3 \mathrm{~cm}^{2}$, and their length was taken to 20 radiation lengths giving a total length of $18.6 \mathrm{~cm}$, thus more than $99.9 \%$ of the photon energy is absorbed in the crystals, and a typical electromagnetic shower is contained in 9 adjacent blocks, with approximately $90 \%$ of the energy deposited in the central block.

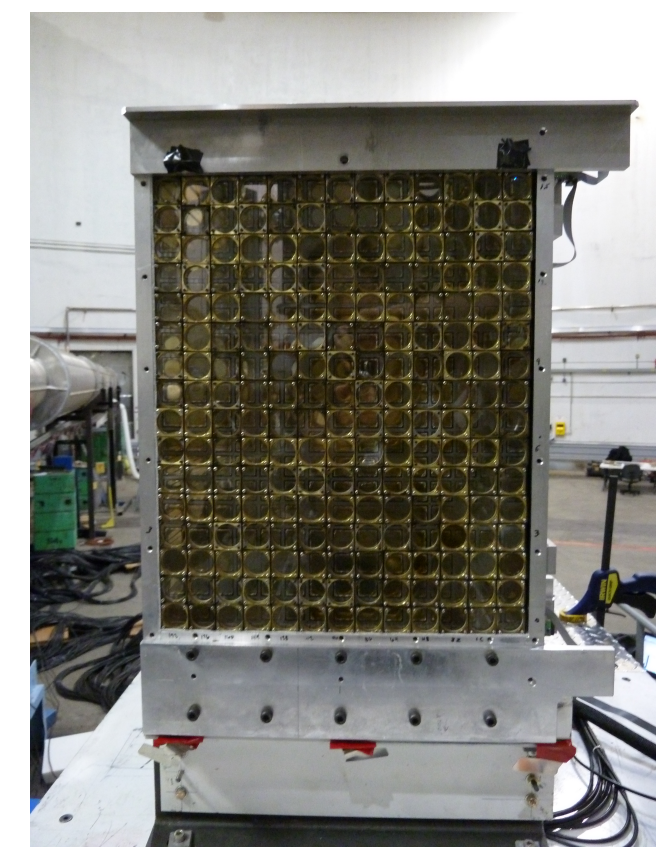

Figure 2.6: Front view of the calorimeter

$\mathrm{PbF}_{2}$ emits only light from Čerenkov process, around 1000 Čerenkov photons are emitted per $\mathrm{GeV}$. This has the advantage of being insensitive to background particles of $\beta<\frac{c}{n}=\frac{1}{1.8}=0.56$, corresponding to electrons lower than $0.6 \mathrm{MeV}$ which cuts a part of the low energy background. Also it has a quick response, making easier pulse separation in case of pile-up.

The calorimeter was designed modularly, each block was coupled to a PMT (Hamamatsu R7700) using a tightening fixture in brass. Each block is wrapped in Tyvek ${ }^{\circledR}$ (internal wrapping) and Tedlar $^{\circledR}$ (external wrapping). A small bus was designed for each calorimeter column in order to supply the HV, the DC current for the amplifier, the monitoring of the anode current and a support for the signal cable. 


\begin{tabular}{|c|c|c|c|c|c|c|c|c|c|c|c|c|}
\hline 15 & 31 & 47 & 63 & 79 & 95 & 111 & 127 & 143 & 159 & 175 & 191 & 207 \\
\hline 14 & 30 & 46 & 62 & 78 & 94 & 110 & 126 & 142 & 158 & 174 & 190 & 206 \\
\hline 13 & 29 & 45 & 61 & 77 & 93 & 109 & 125 & 141 & 157 & 173 & 189 & 205 \\
\hline 12 & 28 & 44 & 60 & 76 & 92 & 108 & 124 & 140 & 156 & 172 & 188 & 204 \\
\hline 11 & 27 & 43 & 59 & 75 & 91 & 107 & 123 & 139 & 155 & 171 & 187 & 203 \\
\hline 10 & 26 & 42 & 58 & 74 & 90 & 106 & 122 & 138 & 154 & 170 & 186 & 202 \\
\hline 9 & 25 & 41 & 57 & 73 & 89 & 105 & 121 & 137 & 153 & 169 & 185 & 201 \\
\hline 8 & 24 & 40 & 56 & 72 & 88 & 104 & 120 & 136 & 152 & 168 & 184 & 200 \\
\hline 7 & 23 & 39 & 55 & 71 & 87 & 103 & 119 & 135 & 151 & 167 & 183 & 199 \\
\hline 6 & 22 & 38 & 54 & 70 & 86 & 102 & 118 & 134 & 150 & 166 & 182 & 198 \\
\hline 5 & 21 & 37 & 53 & 69 & 85 & 101 & 117 & 133 & 149 & 165 & 181 & 197 \\
\hline 4 & 20 & 36 & 52 & 68 & 84 & 100 & 116 & 132 & 148 & 164 & 180 & 196 \\
\hline 3 & 19 & 35 & 51 & 67 & 83 & 99 & 115 & 131 & 147 & 163 & 179 & 195 \\
\hline 2 & 18 & 34 & 50 & 66 & 82 & 98 & 114 & 130 & 146 & 162 & 178 & 194 \\
\hline 1 & 17 & 33 & 49 & 65 & 81 & 97 & 113 & 129 & 145 & 161 & 177 & 193 \\
\hline 0 & 16 & 32 & 48 & 64 & 80 & 96 & 112 & 128 & 144 & 160 & 176 & 192 \\
\hline
\end{tabular}

Table 2.1: Schematic view of the backside of the calorimeter, showing block numbers.

In order to balance the PMTs gain cosmic rays were used. Two scintillator paddles were placed on top of the calorimeter to generate a cosmics trigger. The stop was generated by the scintillators and validated by the calorimeter trigger. In order to select cosmics going through the whole calorimeter, a coincidence between the top row and the bottom row was also required by the calorimeter trigger. Such high energy cosmic rays leave energy at the minimum ionizing value giving a well defined energy loss peak in each block allowing to balance the gain.

\subsection{Data acquisition}

The electromagnetic calorimeter data acquisition system has been designed to store the deposited energy of hitting particles as a function of an electric signal proportional to the current of the PMTs attached to each one of its conforming blocks. These "wave signals" encode the energy deposited on each block for a time window of $128 \mathrm{~ns}$ since the issue of the trigger from the HRS. Two main systems are involved in this process, the "Analog Ring Sampler", a device which stores the current variation of the PMTs for 128ns, and the calorimeter trigger.

\subsubsection{The Analog Ring Sampler}

The Analog Ring Sampler is composed of 128 capacitors continuously sampling PMT signals from the calorimeter [54]. The charge of each capacitor is proportional to the value of the input signal. The incoming signals are continuously switched at a clock 
frequency of $1 \mathrm{GHz}$ as shown in Figure 2.7. As long as no trigger is issued the samples get continuously overwritten. When a trigger is issued the switching is stopped and the previous $128 n s$ remain stored on the capacitors (i.e. they are not overwritten).
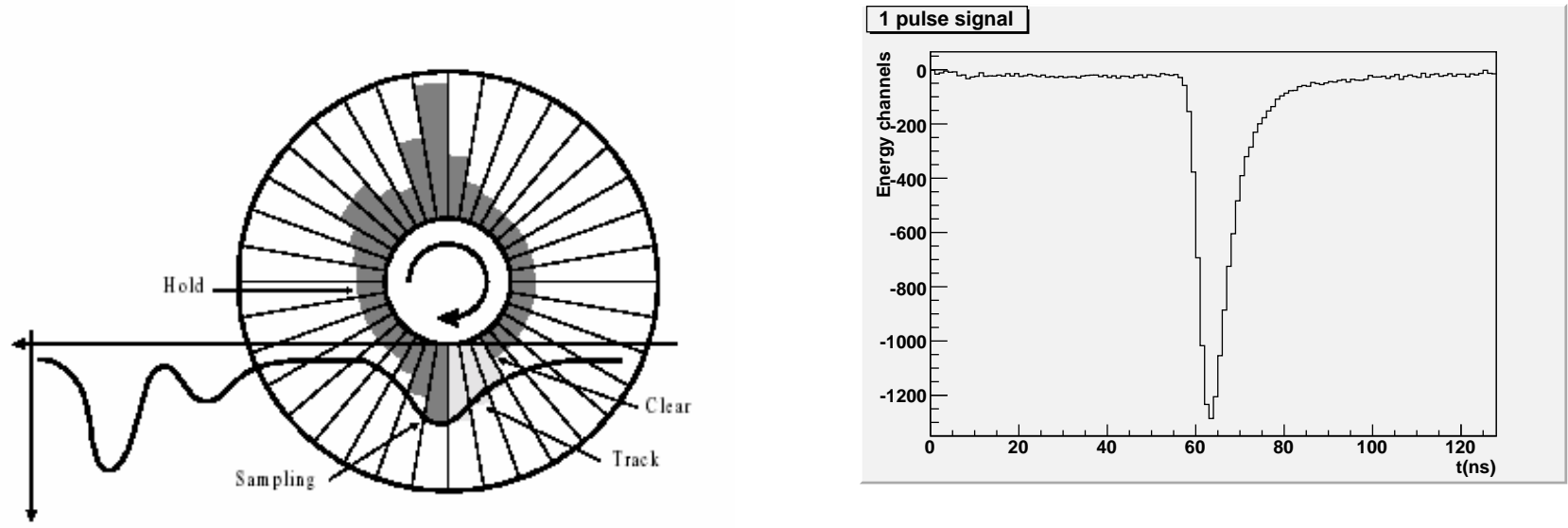

Figure 2.7: Digitalization of the charge stored in the ARS capacitors.

The digitalization allows the recording of the signal of each channel for every event over $128 n s$ as in a digital oscilloscope. The main purpose of this digitalization is to be able to identify pile-up events and recognize the various signals in order to treat them separately.

\subsubsection{Trigger}

During the experiment the spectrometer trigger was composed by the scintillator plane S2 and the Cerenkov detector. S2 defined $t=0$ for the calorimeter $128 \mathrm{~ns}$ time window. Once the spectrometer issues the trigger signal, the ARS stores the charge proportional to the $\mathrm{PbF}_{2}$ calorimeter blocks input signal, as explained in the previous section. At the same time 12-bit-flash-ADCs integrate the signal of each PMT attached to each block composing the calorimeter. The trigger module computes the sum of these ADC values for every set of $2 \times 2$ neighboring blocks. This is done since the electromagnetic cascade is usually contained in 9 adjacent blocks, and a one block threshold would not be effective.

This computation takes as much as 340 ns as shown in Figure 2.8. During this "decision time" only the ARS corresponding to $2 \times 2$ sets above a threshold will be selected as fit for recording. Notice that while the calorimeter trigger only takes $340 \mathrm{~ns}$ in selecting the signals for data storing, it has a dead time of $500 \mathrm{~ns}$. The difference between both times is employed in resetting the trigger sampling system. The digitalization and transfer of the data imply a dead time of about $128 \mu \mathrm{s}$, in the case of at least one set of $2 \times 2$ blocks being above the threshold. This time is larger than that needed for data transferring and 


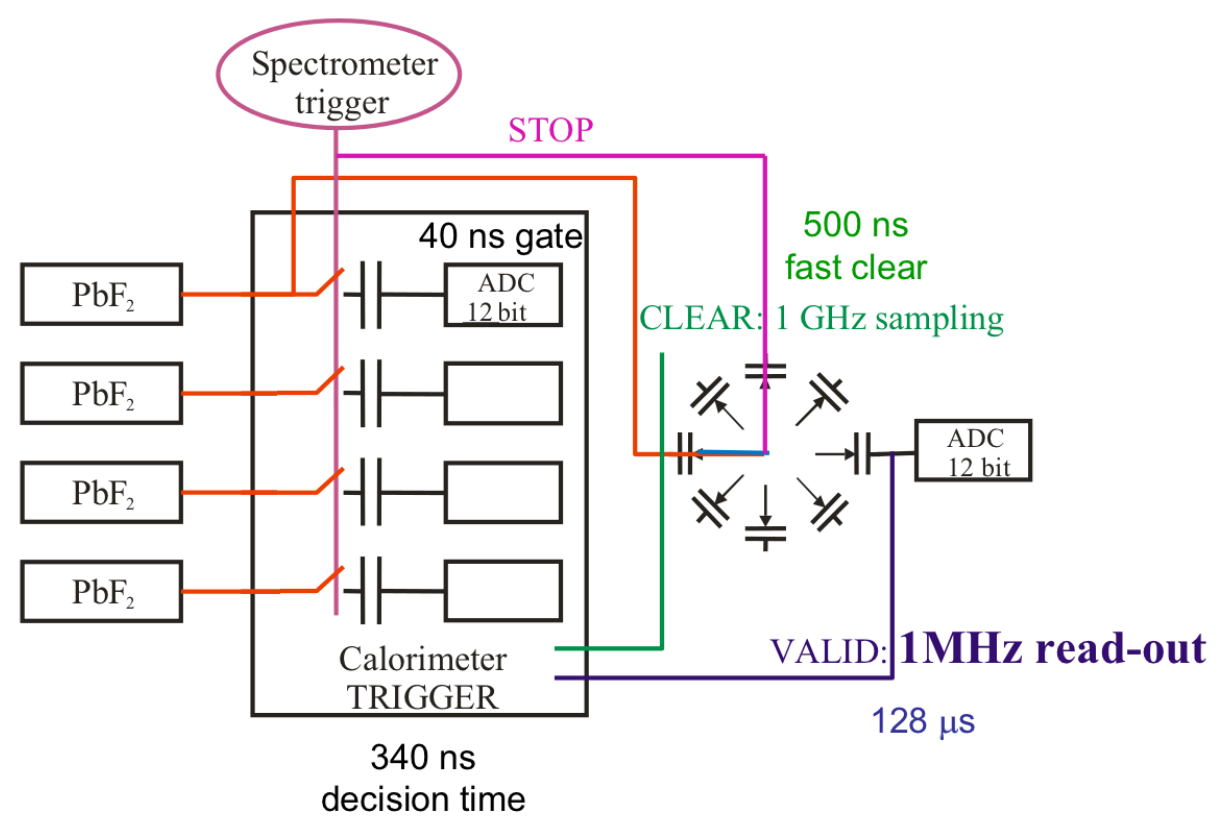

Figure 2.8: Schematic layout of the calorimeter data acquisition.

trigger resetting in the HRS.

Unfortunately, the calorimeter trigger was only operational during the last week of the experiment. During most of the time the data from the calorimeter were stored whenever the spectrometer trigger issued a signal.

\subsubsection{Data acquisition in Hall A}

The general data-acquisition systems in Hall A use CODA (CEBAF On-line Data Acquisition System) developed by the JLab data-acquisition group [55]. CODA is a toolkit of distributed-software components from which data-acquisition systems of varying degrees of complexity can be built. Supported hardware elements are mainly commercially available electronics.

Custom hardware elements made at JLab include the trigger supervisor which synchronizes the read-out of the front-end crates and handles the dead-time logic of the system [56]. The most important custom software components of CODA are the read-out controller (ROC) which runs on the front-end crates, the event builder (EB) and event recorder (ER) which run on a Unix or Linux workstation, the event transfer (ET) system which allows distributed access to the data on-line or insertion of data from user processes, 
and finally the RunControl process, from which users can select different experimental configurations, start and stop runs, as well as reset and monitor CODA components.

For each event, which corresponds to a trigger accepted by the trigger supervisor, data are gathered from the front-end boards by the ROC component, which buffers the data in memory and sends these buffers via the network to the EB running on a workstation. The EB builds events from fragments sent by the various ROCs and passes them to the ER which writes data to a local disk. The data are subsequently written to tapes and erased from disk typically after one day. Using the ET system, various additional pieces of data are inserted into the data stream every few seconds from the control system, scalers, or text files of information. In addition, the ET system is used by analysis clients to obtain a random sample of data in real-time anywhere on the network.

The Hall A physics data analysis model is straightforward. Raw data from the data acquisition (DAQ) are decoded and analyzed by an event-processing program. The event processor creates data summary files that contain raw and computed data, typically in ntuple and histogram format. Diagnostic information as well as final physics results are then extracted from the summary files with the help of interactive analysis tools.

\subsection{Experimental Setup}

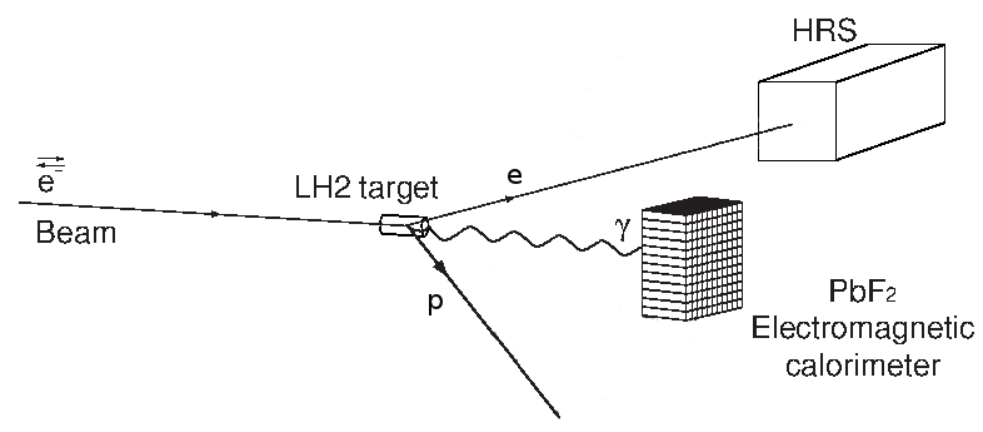

Figure 2.9: Experimental setup. The electron beam interacts with the $\mathrm{LH}_{2} / \mathrm{LD}_{2}$ target, producing an electron, a proton and a photon.

Figure 2.9 shows the schematic layout of the setup for the DVCS experiment E07-007. The electron beam interacts with the $\mathrm{LH}_{2} / \mathrm{LD}_{2}$ target, producing an electron, a proton and a photon. The electron is detected by the High Resolution Spectrometer (HRS), while the photon is detected by the electromagnetic calorimeter. Since the scattered proton is 
not detected, we will identify the events where we had a scattered proton with the variable $M_{X}^{2}$ "Missing Mass", through the energy-momentum conservation relationship:

$$
M_{X(e p \rightarrow e \gamma X)}^{2}=\left(k-k^{\prime}+p-q^{\prime}\right)^{2}
$$

We will select our DVCS events by imposing a cut in $M_{X}^{2}<\left(M_{p}+M_{\pi^{0}}\right)^{2} \simeq 1.15 \mathrm{GeV}^{2}$.

During the time the experiment was running, several calibration sessions took place for the calibration of the electromagnetic calorimeter blocks. For this purpose a different setup was used (figure 2.10). This time the electron beam interacted with a target, producing an electron and a proton in an elastic scattering reaction described as $p+e \rightarrow p^{\prime}+e^{\prime}$. The proton was detected by the HRS, while the electron hit the calorimeter. Since we are studying an elastic reaction, only by measuring the energy-momentum of the detected proton in the HRS we can calculate the energy-momentum of the electron detected by the calorimeter. Then we correlate the response of the signals from the calorimeter to that of the electron energy to calibrate the energy channels.

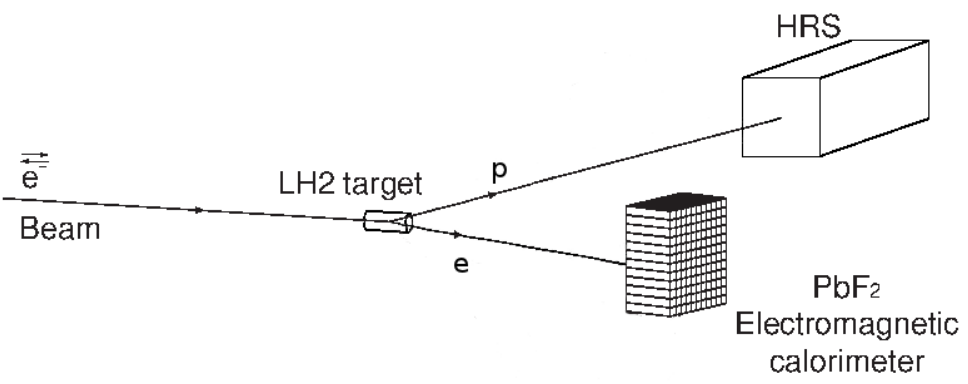

Figure 2.10: Calorimeter calibration setup.

Table 2.2 shows the settings for the different experiment running sessions as well as their names and dates. Kinematics enclose all the different experiment runs with the same indicated settings. $\theta_{L-H R S}$ and $\theta_{\text {Calo }}$ stand for the position angle with respect to the beam line of the left spectrometer and the calorimeter respectively. $P_{L-H R S}$ stands for the momentum central value of the scattered electron detected by the L-HRS. Calo dis. refers to the distance of the center of the calorimeter surface to the target. Notice that it has a different value depending on if a calibrating session or a DVCS session was being conducted. During calibrating sessions, the calorimeter was positioned at a larger distance from the target than in DVCS sessions. This was done in order to have a full coverage of the device, needed for the correct calibration of all its conforming blocks. 
The different angles for $\theta_{L-H R S}$ and $\theta_{\text {Calo }}$ serve to cover different parts of the calorimeter (right, center, left). Table 2.3 shows the statistics collected for each kinematics, including the target employed for each setting [10].

\begin{tabular}{|c|c|c|c|c|c|c|c|}
\hline $\begin{array}{c}\text { Kinematic } \\
\text { name }\end{array}$ & $x_{B}$ & $\begin{array}{c}Q^{2} \\
\left(\mathrm{GeV}^{2}\right)\end{array}$ & $\begin{array}{c}E_{\text {beam }} \\
(\mathrm{GeV})\end{array}$ & $\begin{array}{c}\theta_{L-H R S} \\
(\mathrm{deg})\end{array}$ & $\begin{array}{c}P_{L-H R S} \\
(\mathrm{GeV})\end{array}$ & $\begin{array}{c}\theta_{\text {Calo }} \\
(\mathrm{deg})\end{array}$ & $\begin{array}{c}\text { Calo dis. } \\
(\mathrm{m})\end{array}$ \\
\hline Elastic 1 & - & - & 5.552 & $37.00 / 34.76 / 33.29$ & $2.406 / 2.607 / 2.748$ & 22.6 & 5.5 \\
\hline Elastic 2 & - & - & 4.454 & $40.00 / 37.86 / 36.30$ & $1.981 / 2.131 / 2.245$ & 24.3 & 5.5 \\
\hline Kin 1-low & 0.36 & 1.5 & 3.356 & 36.56 & 1.136 & 14.78 & 1.1 \\
\hline Kin 1-high & 0.36 & 1.5 & 5.552 & 16.37 & 3.332 & 19.39 & 1.1 \\
\hline Kin 2-low & 0.36 & 1.75 & 4.454 & 26.55 & 1.864 & 14.78 & 1.1 \\
\hline Kin 2-high & 0.36 & 1.75 & 5.552 & 18.78 & 2.962 & 16.79 & 1.1 \\
\hline Kin 3-low & 0.36 & 2 & 4.454 & 31.82 & 1.494 & 14.78 & 1.1 \\
\hline Kin 3-high & 0.36 & 2 & 5.552 & 21.49 & 2.591 & 14.78 & 1.1 \\
\hline
\end{tabular}

Table 2.2: Different settings used during the experiment.

\begin{tabular}{|c|c|c|c|}
\hline Kinematic & PAC hours & \% Completed & Target \\
\hline Kin 1-low & 60 & $100 \%$ & $\mathrm{LH}_{2}$ \\
\hline Kin 1-high & 20 & $100 \%$ & $\mathrm{LH}_{2}$ \\
\hline \multirow{2}{*}{ Kin 2-low } & 90 & $100 \%$ & $\mathrm{LH}_{2}$ \\
& 200 & $70 \%$ & $\mathrm{LD}_{2}$ \\
\hline \multirow{2}{*}{ Kin 2-high } & 30 & $100 \%$ & $\mathrm{LH}_{2}$ \\
& 200 & $60 \%$ & $\mathrm{LD}_{2}$ \\
\hline Kin 3-low & 150 & $82 \%$ & $\mathrm{LH}_{2}$ \\
\hline Kin 3-high & 50 & $100 \%$ & $\mathrm{LH}_{2}$ \\
\hline
\end{tabular}

Table 2.3: Statistics collected for each kinematics. 

Chapter

\section{Calorimeter Signal Analysis and Calibration}

Due to the high luminosity of the experiment in Hall A at Jefferson Lab, it is necessary to deal with pile-up and background detections. When there is a detection, signals from the calorimeter are stored for 128ns. By visualizing these time windows, we can observe the photomultiplier current variation as a response to deposited energy: one pulse shape when we have a hit, two pulses (pile-up) or background noise. Therefore, it is necessary to implement an algorithm to extract the amplitude and time of arrival of these pulses, in order to reconstruct the energy and momentum of the resulting photons from DVCS, essential for calculating cross sections. We do so by comparing the signals to reference shapes obtained from calibration sessions. At the same stage it is necessary to correct the time of arrival, taking into account the different set-up distances of the calorimeter during the experiment, differences in cable lengths and relative position of the blocks conforming the apparatus.

Once the amplitude and time of arrival of the signals are known, it is important to select the blocks where the incident particles deposited their energy. We would be taking too much noise contribution into our calculation if we took every block into account. Moreover, we need to know the exact point of impact if we want to obtain the particles momentum. This block selection is performed by a clustering algorithm which selects combinations of blocks as a function of a pre-set energy threshold.

In order to convert signal amplitudes obtained from the waveform analysis into energy units, it was necessary to conduct several calibration sessions, using the elastic reaction $\mathrm{e}+\mathrm{p} \rightarrow \mathrm{e}^{\prime}+\mathrm{p}$ '. In this chapter we will review the waveform and clustering analysis as well as go through the main steps of the calorimeter calibration. 


\subsection{Waveform analysis}

The purpose of the waveform analysis is to obtain the amplitude $a$ and time of arrival $t$ of the calorimeter signals $\left\{x_{i}\right\}$, by fitting those signals to a reference shape $\left\{h_{i}\right\}$. In an ideal event without noise where $t=0$, the amplitude would be the one which minimizes

$$
\chi^{2}=\sum_{i=0}^{127}\left(x_{i}-a h_{i}\right)^{2} .
$$

Here $i$ runs for all the time window samples. But (3.1) is an ideal case, and generally the time of arrival is not known, so the equation above will be transformed into:

$$
\chi^{2}(t)=\sum_{i=0}^{127}\left(x_{i}-a(t) h_{i-t}\right)^{2} .
$$

Amplitudes are calculated analytically for every $t$. Then the reference shape is shifted from its original position and the $\chi^{2}(t)$ from (3.2) is calculated for every $t$ of the time window. We will define the arrival time of the signal as the time where we found the minimum $\chi^{2}(t)$ and so the best fit. The computation of the amplitude in our case is

$$
\frac{\partial \chi^{2}}{a(t)}=-2 \sum_{i=0}^{127}\left(x_{i}-a(t) h_{i-t}\right) h_{i-t}=0,
$$

and therefore

$$
a(t)=\frac{\sum_{i=0}^{127} x_{i} h_{i-t}}{\sum_{i=0}^{127} h_{i-t}^{2}} .
$$

\subsubsection{Reference pulses}

Reference shapes are calculated from the pulses obtained in calibration sessions. For each calibration session we create a reference pulse, for every block, from signals stored for that block. The reference pulse is created using signals with a high response from the photomultipliers (usually above half the energy channels). The first of the selected signals is weighted by its amplitude, and then we begin an iterative process in which we add the rest of the signals to the first one. In each step, a new signal is added to the sum with a weight that starts from 1 , and increases its value in 1 iteration after iteration. The pulses are shifted in time with the aim of superimposing them all. The time of the reference shape is set at the time when most of the signals used for its construction arrived. 


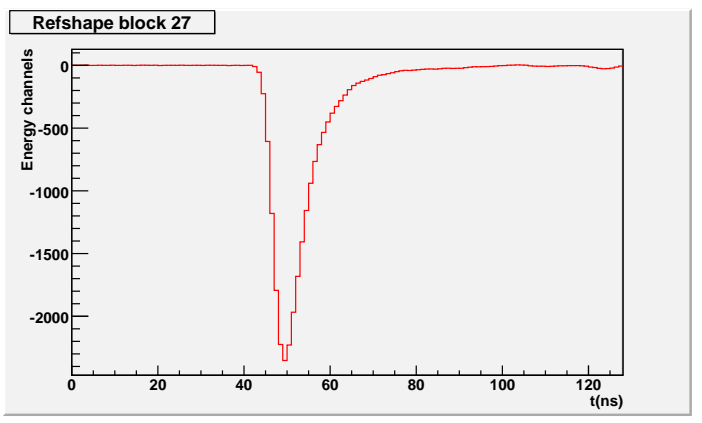

Figure 3.1: Reference shape for block 27. Elastic 2 (17 Nov) calibration.

\subsubsection{Baseline fit}

We analyse the digitized signals to find 3 different cases (noise, one pulse and two pulses) which we will differentiate by the value of the $\chi^{2}$ of our fit, which is defined for each case. When analysing any signal, the first thing we check is whether it is noise or potentially useful data. For this matter we include a baseline $b(t)$ in the definition of our $\chi^{2}$,

$$
\chi^{2}=\sum_{i=\chi_{\min }^{2}}^{\chi_{\max }^{2}}\left(x_{i}-b\right)^{2} \quad \text { where } \quad b=\frac{1}{\left(i_{\max }-i_{\min }\right)} \sum_{i=i_{\min }}^{i_{\max }} x_{i} .
$$

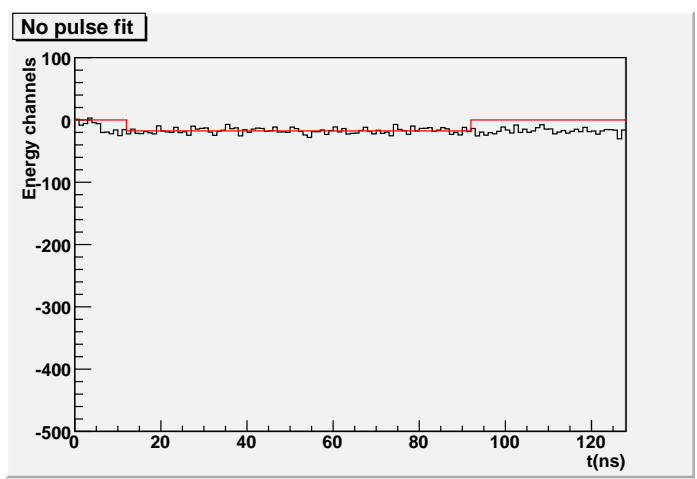

Figure 3.2: Noise from PMTs. $b$ is represented in red. Notice that the initial and final parts of the data window are not included in the analysis window.

Here, $b$ is just the mean value of the signal between $i_{\min }$ and $i_{\max }$ (values of the $128 \mathrm{~ns}$ time window). Note that the index for the $\chi^{2}$ calculation runs from $\chi_{\min }^{2}$ to $\chi_{\max }^{2}$. These 
different analysis time windows are meant to speed up the computation by only considering the portion of the $128 \mathrm{~ns}$ time window where most of the information is expected to be found. If the $\chi^{2}$ is smaller than a $\chi_{0}^{2}$ threshold (table 3.2 shows all the bounds and thresholds for the analysis parameters. Criteria for threshold selection is explained in section 3.1.6), the baseline will be considered good and the signal will be discarded. Otherwise a one pulse fit will be performed as shown in the following case.

\subsubsection{One pulse fit}

Most of the useful information is obtained from one pulse fits. In this case, we include the baseline in our $\chi^{2}$ definition in order to minimize the impact of background noise.

$$
\chi^{2}\left(t_{1}\right)=\sum_{i=\chi_{\min }^{2}}^{\chi_{\max }^{2}}\left(x_{i}-a_{1}\left(t_{1}\right) h_{i-t_{1}}-b\left(t_{1}\right)\right)^{2} .
$$

In this case, the baseline fit does not have the same definition as in the previous case. It is calculated analytically along with the amplitude $a\left(t_{1}\right)$, by selecting the values that minimize our $\chi^{2}$. The minimization yields the set of equations

$$
\begin{aligned}
& \frac{\partial \chi^{2}}{\partial a_{1}}=-2 \sum_{i=i_{\min }}^{i_{\max }}\left(x_{i}-a_{1}\left(t_{1}\right) h_{i-t_{1}}-b\left(t_{1}\right)\right) h_{i-t_{1}}, \\
& \frac{\partial \chi^{2}}{\partial b}=-2 \sum_{i=i_{\min }}^{i_{\max }}\left(x_{i}-a_{1}\left(t_{1}\right) h_{i-t_{1}}-b\left(t_{1}\right)\right)
\end{aligned}
$$

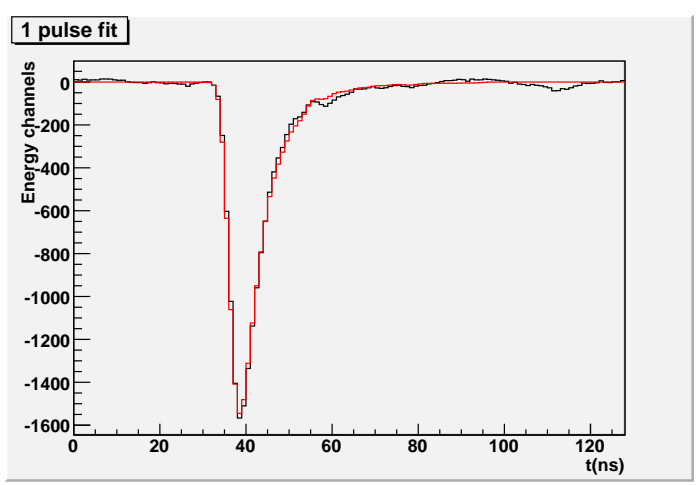

Figure 3.3: One pulse fit.

which can be displayed as a combination of matrices, 


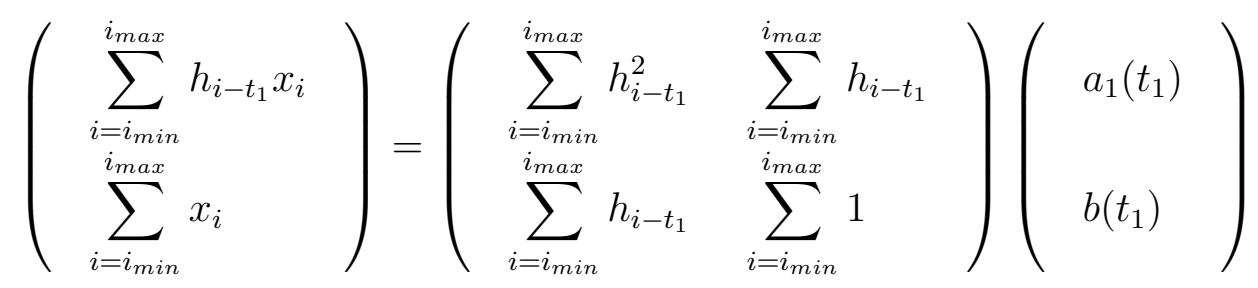

Here again, we use a threshold on the $\chi^{2}$ to check the quality of our fit. If it is smaller than $\chi_{1}^{2}$, this fit will be considered good and we will save all information such as amplitude, time of arrival, baseline, and $\chi^{2}$ values.

\subsubsection{Two pulse fit}

If the one pulse fit has proven inadequate, we try searching for a second pulse within the bounds of the time window, to avoid losing information from pile-up events. We modify the definition of the $\chi^{2}$ to include a second amplitude $a_{2}\left(t_{1}, t_{2}\right)$,

$$
\chi^{2}\left(t_{1}, t_{2}\right)=\sum_{i=\chi_{\min }^{2}}^{i=\chi_{\max }^{2}}\left(x_{i}-a_{1}\left(t_{1}, t_{2}\right) h_{i-t_{1}}-a_{2}\left(t_{1}, t_{2}\right) h_{i-t_{2}}-b\left(t_{1}, t_{2}\right)\right)^{2} .
$$

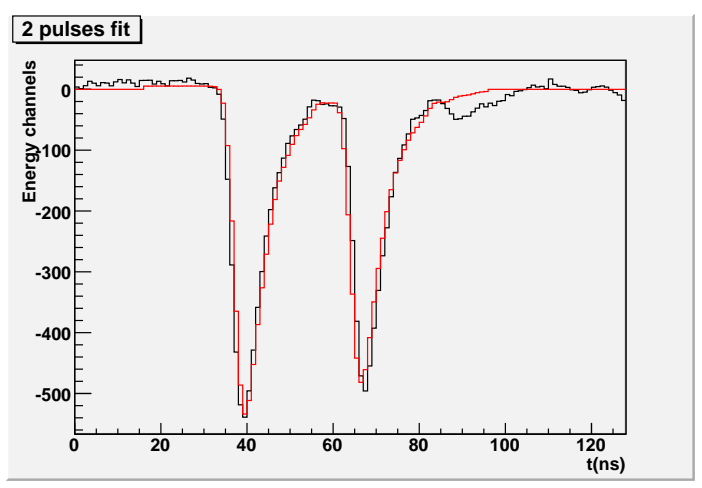

Figure 3.4: Two pulse fit.

There is no further waveform analysis at this point, but if time separation between the two pulses is below $4 n s$ we will keep the result from the one pulse fit. Below this threshold we can't differentiate the signals, they overlap. Again, the minimization of the $\chi^{2}$ yields a set of equations that we express in a matricial form. 


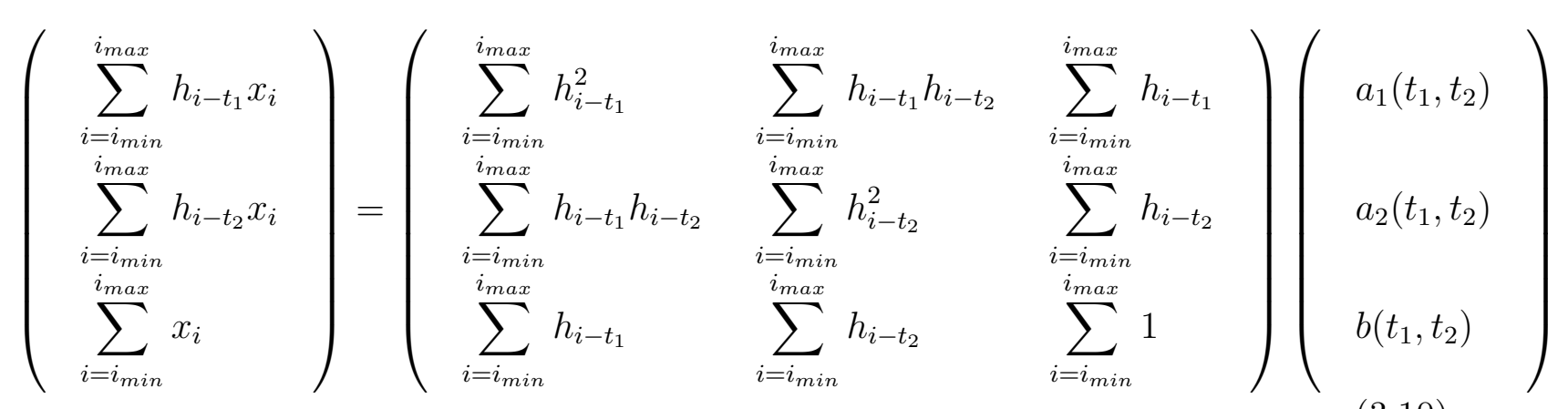

Unfortunately, there is a problem when introducing a baseline in a two pulse fit. Sometimes the baseline will have a higher value than that of the highest peak of the signal, and the amplitudes that minimize the $\chi^{2}$ will be negative (thus rendering false data). In order to avoid this issue it was decided that the two pulse fits would be performed without including a baseline. We rewrite our matrices like

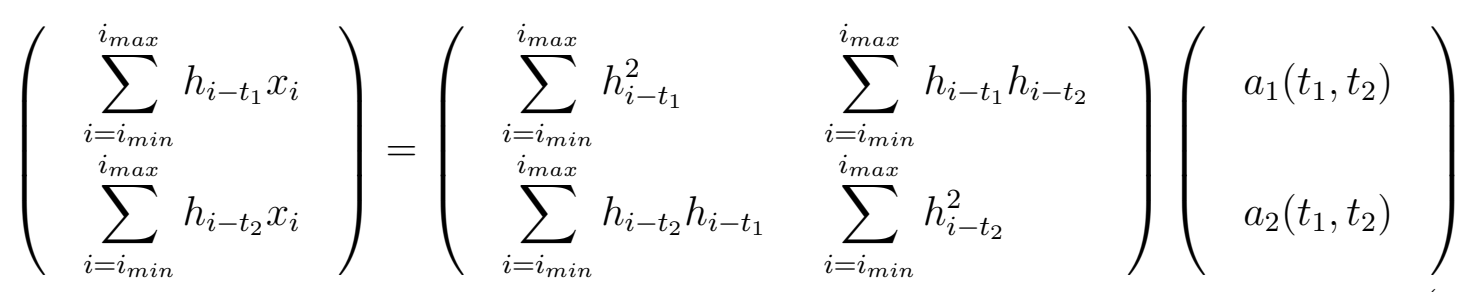

It is unusual for an electronic signal to saturate, but it happens. Even if this is not likely to happen we cannot accept events with saturating signals. For calibration purposes, whenever any block showed this problem, the whole event was discarded. During the experiment the saturation threshold was too high to affect the data.

\subsubsection{Arrival time}

The arrival time is an important element to associate different block signals to one another, since the electromagnetic shower unleashed by hitting particles is usually contained in nine or more blocks. This time of arrival will be given with respect to the time assigned to the reference shape of the block in question plus a small correction.

In a preliminary analysis we searched for the time of arrival for most of the signals. We observed that for each block, this variable behaved as a Gaussian distribution. We fitted this 208 distributions to extract the 208 time values we then associated to the reference shapes $(t=0)$. Afterward, we fitted the signals of the experimental runs (not from calibration sessions) using the reference shapes, and again searched for the time of 
arrival for most of the signals. Here, once more, we found Gaussian distributions [57]. Since this analysis is conducted using reference shapes what we obtain is the difference in time between the pulse with respect to the reference shapes (one of the reasons of this difference is that reference shapes are obtained from calibrating sessions where the calorimeter was further positioned than in experimental sessions). We will refer to this variable as $t_{c o r r}$ (time correction, usually a few $n s$ ).

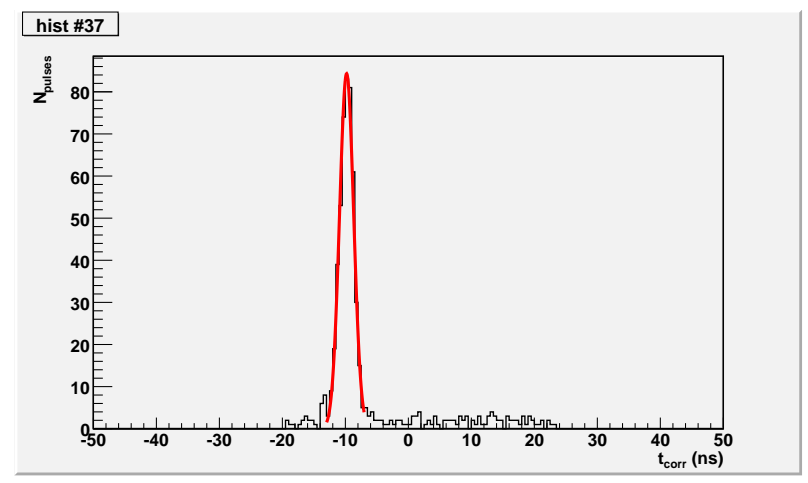

Figure 3.5: Gaussian fit of time difference between a pulse and the reference shape.

Since data are digitized at $1 \mathrm{GHz}$ (1ns sample), we can't experimentally have a better resolution than $1 n s$, but it is possible to increase this resolution for the arrival time through an interpolation using $\chi_{\min }^{2}$ (the minimum $\chi^{2}(t)$, thus determining the time of arrival),$\chi_{t+1}^{2}$ and $\chi_{t-1}^{2}$ (one $n s$ after and before $\chi_{\min }^{2}$ ). To do so we introduce a parabolic relation between time and $\chi^{2}(t)$,

$$
\chi^{2}(t)=a t^{2}+b t+c .
$$

Then we minimize the $\chi^{2}(t)$ to find $t_{\min }$

$$
\frac{\partial \chi^{2}(t)}{\partial t}=2 a t_{\min }+b=0 \quad t_{\min }=\frac{-b}{2 a} .
$$

We can easily obtain the following equations using (3.12)

$$
\begin{gathered}
\chi^{2}(0)=c \\
\chi^{2}(1)=a+b+c, \\
\chi^{2}(-1)=a-b+c .
\end{gathered}
$$

and combine them to reach the final expression for $t_{\min }$. The expression for the time of arrival with the interpolation is related to that without the interpolation, $t\left(\chi_{\min }^{2}\right)$, in the general equation 


$$
t_{\text {min }}=t\left(\chi_{\text {min }}^{2}\right)+\frac{\chi_{t-1}^{2}-\chi_{t+1}^{2}}{2\left(\chi_{t+1}^{2}+\chi_{t-1}^{2}-2 \chi_{\min }^{2}\right)} .
$$

This correction can reach up to $\pm 0.5 n s$ of the arrival time. Once we have applied all time corrections we can repeat the waveform analysis, including all corrections and checking that the arrival time of most of the pulses of each block for each run of every kinematic is near zero or 1 ns at worst. Figure 3.6 shows the values of this parameter for four different blocks $\left(\right.$ black $=$ Kin 1-low; red $=$ Kin 1-high; green $=$ Kin 2-low $\left(\mathrm{LD}_{2}\right)$; blue = Kin 2-low; yellow $=$ Kin 2-high $\left(\mathrm{LD}_{2}\right)$; magenta $=$ Kin 2-high; light blue = Kin 3-low; olive $=$ Kin 3-high):
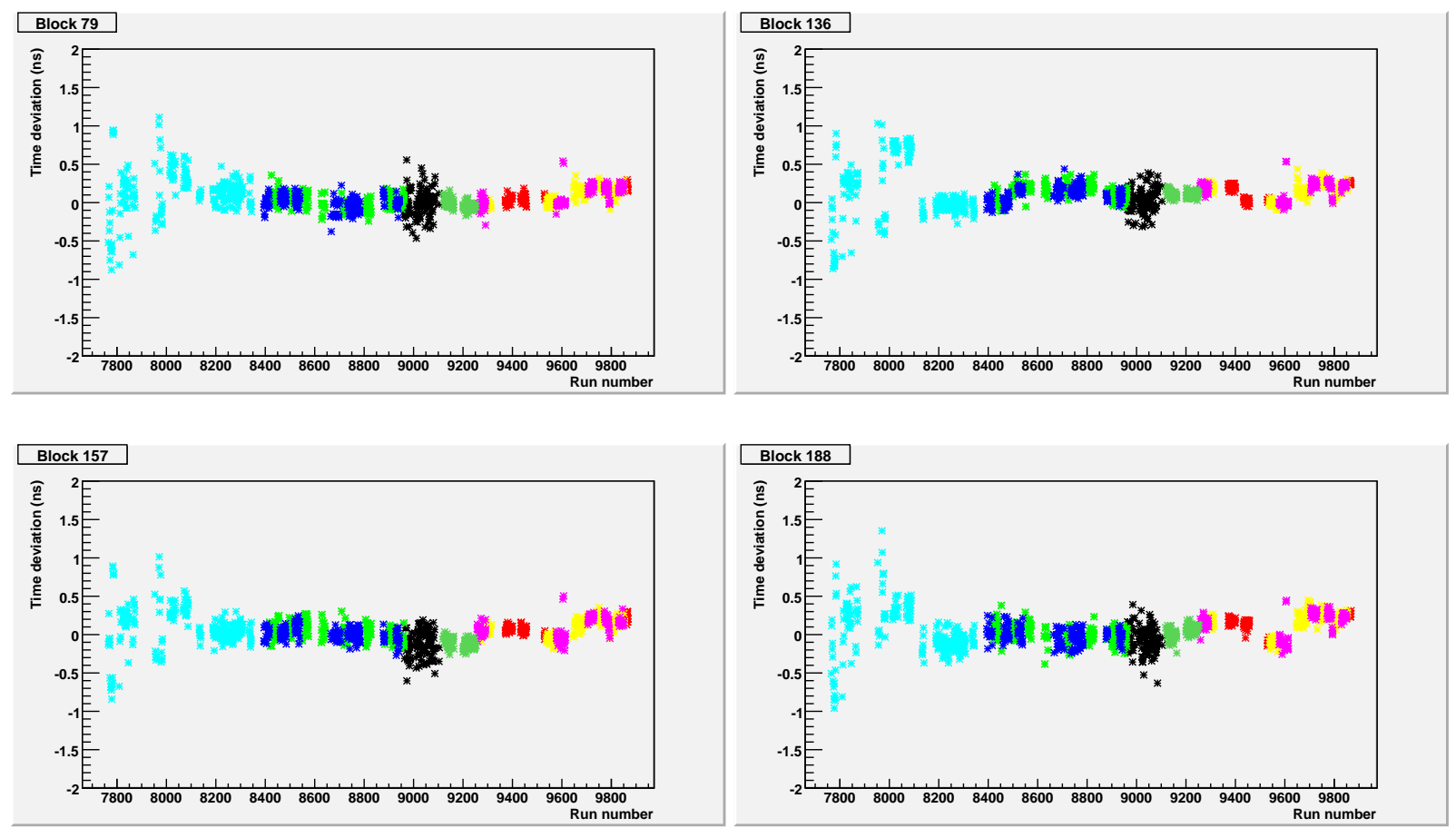

Figure 3.6: Time of arrival for most of the pulses for each run for four different blocks. Runs in the left (light blue) belong to an early stage of the experiment, when some issues concerning block malfunction aroused. This is addressed later in this chapter (3.3.3).

\subsubsection{Analysis parameters}

The selection of an appropriate fitting threshold is important to perform a fit that adjusts the type of pulse we are dealing with. It will also affect the speed of the analysis. This speed can be increased by introducing a set of parameters to extract valuable information from the time window without having to process the whole lot of it. 


\section{One pulse threshold}

Signals that don't reach a minimum energy, $E_{0}$, after a baseline fit are discarded and therefore no one pulse fit is performed. We saw that the waveform analysis is done by minimizing the $\chi^{2}$ of the signal fit, but this is not a variable we are familiar with. We evaluate $E_{0}$ in terms of $\chi^{2}$ by using the reference shapes.

$$
\chi_{r e f}^{2}=\sum_{i=\chi_{\min }^{2}}^{\chi_{\max }^{2}} h_{i}^{2} .
$$

$\chi_{\text {ref }}^{2}$ is basically the area covered by the reference shape in energy channels. Since a reference pulse has an amplitude, $a_{1}=1$, its energy is the value of its associated calibration coefficient in $\mathrm{GeV}$ (see eq. (3.18)). So, for a pulse with energy $E_{0}$ we have

$$
\chi_{0}^{2}=\sum_{i=0}^{127}\left(\frac{E_{0}}{C_{i}} h_{r e f}(i)\right)^{2} .
$$

Here, $C_{i}$ stands for the calibration coefficients. Now we can compare the $\chi^{2}$ from any baseline fit with $\chi_{0}^{2}$. If $\chi^{2}<\chi_{0}^{2}$ the signal is discarded. This means that after performing the baseline fit, if the $\chi^{2}$ differs in more than a value associated to an energy $E_{0}$ we will look for a pulse. In order to decide which would be the most appropriate $E_{0}$ we did some testing with different values, and studied the evolution of the calorimeter resolution using calibration runs.

\begin{tabular}{c|c|c} 
Threshold $(\mathrm{MeV})$ & Coefficients variation & Resolution \\
\hline 0 & - & $3.07 \%$ \\
21 & $\sim 0.3 \%$ & $3.06 \%$ \\
42 & $\sim 3 \%$ & $3.35 \%$ \\
63 & $\sim 5 \%$ & $3.86 \%$
\end{tabular}

Table 3.1: Variation of calorimeter resolution with the one pulse threshold. The computation of the resolution is addressed in section 3.3.2.

Table 3.1 shows the variation induced in the calibration coefficients when the one pulse threshold is altered. The resolution of the calorimeter is recalculated using the new coefficients and also displayed in the table above. $21 \mathrm{MeV}$ was selected as threshold, $E_{0}$, due to the minimum variation on the calorimeter resolution.

\section{Two pulse threshold}

$\chi_{1}^{2}$ has a value of $100 \mathrm{MeV}$ in energy units. This means that when a 1 pulse is performed and our $\chi^{2}$ shows a discrepancy of $100 \mathrm{MeV}$ or more with the fit, a 2 pulses fit will be 
conducted. Since the average energy of the photons hitting the calorimeter is about 2.4 $\mathrm{GeV}$, the threshold is set at around $4 \%$ of this energy. Setting a lower threshold would be possible, but it would increase the analysing time (decreasing the threshold by half of its value increases the analysing time by $30 \%$ approximately). This threshold is to make sure we have two pulses when we perform a two fit pulse.

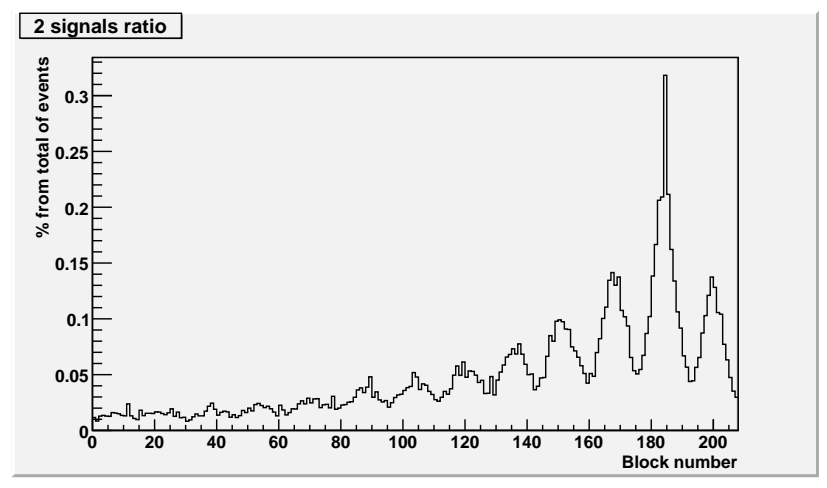

Figure 3.7: Events with two pulses per block. Both signals $>30 \mathrm{MeV}$.

The data shown on Figure 3.7 belong to run 9123 (Kin 3-high $\mathrm{LH}_{2}$ ). The histogram shows the amount of events with two pulses we find for every block as a percentage of the total of events stored by the calorimeter. We find $8.4 \%$ of events with at least one block with two signals. The nearer the blocks are to the beam the more 2 pulses signals are collected. It is easy to recognize the pattern of the columns of blocks in the calorimeter. Blocks located in the middle of these columns have more statistics.

\section{Parameters summary}

The values for the parameters employed in the waveform analysis are summarized in table 3.2. Along with the table a brief description of all of them is given.

- Analysis window: is delimited by $i_{\min }$ and $i_{\max }$ to dispose of the first and last $n s$ of the data window. These parts carry no useful information. We estimated that all the valuable information is contained in a $80 \mathrm{~ns}$ window, since the usual width of a pulse is about $30 n s$ (we take into account that we might detect two pulses in the same window). We adjust the signals taking care not to reach the borders of the data window.

- $\chi^{2}$-window: the $\chi^{2}$ is used to test the quality of our fits, but it is only calculated in a $40 n s$ window around the minimum value of the reference shape $\left(r e f_{\text {min }}\right.$, PMT pulses are negative). This window should be enough to cover the pulse in its whole. 


\begin{tabular}{|c|c|c|}
\hline Analysis window & {$\left[i_{\min }, i_{\max }\right]$} & $\begin{array}{l}i_{\min }=\operatorname{Max}(5 n s, 20 n s+\text { tcorr }) \\
i_{\max }=\operatorname{Min}(123 n s, 100 n s+\text { tcorr })\end{array}$ \\
\hline$\chi^{2}$-window & {$\left[\chi_{\min }^{2}, \chi_{\max }^{2}\right]$} & $\begin{array}{l}\chi_{\min }^{2}=\operatorname{Max}\left(5 n s, r e f_{\min }-20 n s+\text { tcorr }\right) \\
\chi_{\max }^{2}=\operatorname{Min}\left(123 n s, \text { re } f_{\min }+20 n s+\text { tcorr }\right)\end{array}$ \\
\hline 1st-window & {$\left[t_{1}^{\min }, t_{1}^{\max }\right]$} & $\begin{array}{l}t_{1}^{\text {min }}=-20 n s+t c o r r \\
t_{1}^{\text {max }}=25 n s+t c o r r\end{array}$ \\
\hline 2nd-window & {$\left[t_{2}^{\min }, t_{2}^{\max }\right]$} & $\begin{array}{l}t_{2}^{\min }=-40 n s+t c o r r \\
t_{2}^{\max }=40 n s+t c o r r\end{array}$ \\
\hline Resolution & $\Delta \tau$ & $4 n s$ \\
\hline 0 -pulse $\chi^{2}$ threshold & $\overline{\chi_{0}^{2}}$ & $21 \mathrm{MeV}$ \\
\hline 1-pulse $\chi^{2}$ threshold & $\chi_{1}^{2}$ & $100 \mathrm{MeV}$ \\
\hline
\end{tabular}

Table 3.2: Analysis parameters and their values

- 1st-window: this window puts a limit in the time of arrival of pulses to be fitted. If the time of arrival of a pulse is below $-20 n s$ or above $+25 n s$ of that expected the signal will be discarded.

- 2nd-window: same as 1st-window but the window is broadened in order to find piled up events.

- Resolution: If the separation between 2 pulses piled up is less than this parameter we will consider them as one pulse.

- $\chi^{2}$ thresholds: We use these thresholds to select the type of fitting we will perform.

\subsection{Impact blocks selection, Clustering}

When reconstructing the detected photon energy, it is important to select only the blocks in which energy was deposited, since most of them will have collected just noise. If we included all the blocks in the calculation, we would be overestimating the value of the deposited energy. One way of determining the impact point is to set an energy threshold in the signals amplitude for each block. The problem with this method is that the photon lays most of its energy in just one block (about 90\%) while the other 10\% is deposited in the neighboring blocks. If we used this method, we would be underestimating the value of the photon energy. A better way to workaround this issue is to set an energy threshold to the energy sum of four adjacent blocks. An algorithm calculates this sum for every possible combination of four adjacent blocks in the calorimeter. For example, Figure 3.8 shows the scheme of a corner of the calorimeter, where we can see in blue, red and yellow, different possible combinations of four adjacent blocks (some combinations overlap). 


\begin{tabular}{|c|c|c|c|c|c|c}
\hline 15 & 31 & 47 & 63 & 79 & 95 & 111 \\
\hline 14 & 30 & 46 & 62 & 78 & 94 & 110 \\
\hline 13 & 29 & 45 & 61 & 77 & 93 & 109 \\
\hline 12 & 28 & 44 & 60 & 76 & 92 & 108 \\
\hline 11 & 27 & 43 & 59 & 75 & 91 & 107 \\
\hline 10 & 26 & 42 & 58 & 74 & 90 & 106 \\
\hline 9 & 25 & 41 & 57 & 73 & 89 & 105 \\
\hline 8 & 24 & 40 & 56 & 72 & 88 & 104 \\
\hline 7 & 23 & 39 & 55 & 71 & 87 & 103 \\
\hline 6 & 22 & 38 & 54 & 70 & 86 & 102 \\
\hline 5 & 21 & 37 & 53 & 69 & 85 & 101 \\
\hline
\end{tabular}

\begin{tabular}{|c|c|c|c|c|c|c}
\hline 15 & 31 & 47 & 63 & 79 & 95 & 111 \\
\hline 14 & 30 & 46 & 62 & 78 & 94 & 110 \\
\hline 13 & 29 & 45 & 61 & 77 & 93 & 109 \\
\hline 12 & 28 & 44 & 60 & 76 & 92 & 108 \\
\hline 11 & 27 & 43 & 59 & 75 & 91 & 107 \\
\hline 10 & 26 & 42 & 58 & 74 & 90 & 106 \\
\hline 9 & 25 & 41 & 57 & 73 & 89 & 105 \\
\hline 8 & 24 & 40 & 56 & 72 & 88 & 104 \\
\hline 7 & 23 & 39 & 55 & 71 & 87 & 103 \\
\hline 6 & 22 & 38 & 54 & 70 & 86 & 102 \\
\hline 5 & 21 & 37 & 53 & 69 & 85 & 101
\end{tabular}

Figure 3.8: Blue, red, yellow: examples of possible combinations of four adjacent blocks.

Imagine that in our example all the colored combinations from both pictures were above the energy threshold. In this case we would select all the blocks belonging to these combinations as impact blocks:

\begin{tabular}{|c|c|c|c|c|c|c}
\hline 15 & 31 & 47 & 63 & 79 & 95 & 111 \\
\hline 14 & 30 & 46 & 62 & 78 & 94 & 110 \\
\hline 13 & 29 & 45 & 61 & 77 & 93 & 109 \\
\hline 12 & 28 & 44 & 60 & 76 & 92 & 108 \\
\hline 11 & 27 & 43 & 59 & 75 & 91 & 107 \\
\hline 10 & 26 & 42 & 58 & 74 & 90 & 106 \\
\hline 9 & 25 & 41 & 57 & 73 & 89 & 105 \\
\hline 8 & 24 & 40 & 56 & 72 & 88 & 104 \\
\hline 7 & 23 & 39 & 55 & 71 & 87 & 103 \\
\hline 6 & 22 & 38 & 54 & 70 & 86 & 102 \\
\hline 5 & 21 & 37 & 53 & 69 & 85 & 101 \\
\hline
\end{tabular}

\begin{tabular}{|c|c|c|c|c|c|c}
\hline 15 & 31 & 47 & 63 & 79 & 95 & 111 \\
\hline 14 & 30 & 46 & 62 & 78 & 94 & 110 \\
\hline 13 & 29 & 45 & 61 & 77 & 93 & 109 \\
\hline 12 & 28 & 44 & 60 & 76 & 92 & 108 \\
\hline 11 & 27 & 43 & 59 & 75 & 91 & 107 \\
\hline 10 & 26 & 42 & 58 & 74 & 90 & 106 \\
\hline 9 & 25 & 41 & 57 & 73 & 89 & 105 \\
\hline 8 & 24 & 40 & 56 & 72 & 88 & 104 \\
\hline 7 & 23 & 39 & 55 & 71 & 87 & 103 \\
\hline 6 & 22 & 38 & 54 & 70 & 86 & 102 \\
\hline 5 & 21 & 37 & 53 & 69 & 85 & 101 \\
\end{tabular}

Figure 3.9: Violet: selected impact blocks. Red: local maxima.

The "clustering" algorithm, created to form "clusters" (each being a combination of blocks which determine the zone of impact of a particle), then searches for the local maxima of each of the combination of selected impact blocks (Figure 3.9). Then, the algorithm checks the amplitude of the blocks neighboring the local maxima, and forms a cluster by adding every block with a lower amplitude. This process is repeated for every block that neighbors any block already added to the cluster. In the case of our example this last step is not very significant since the two different zones are far from each other, but it proves useful when determining the sizes of close or overlapping impact zones. The exact impact point is computed by assigning a weight, $w_{i}$, to each block of the cluster,

$$
x=\frac{\sum_{i} w_{i} x_{i}}{\sum_{i} w_{i}} \quad w_{i}=\max \left\{0,\left[W_{0}+\ln \left(\frac{E_{i}}{E}\right)\right]\right\} .
$$

where $x$ is the impact point, $x_{i}$ the position of the center of each block and

$$
E_{i}=C_{i} A^{i} \quad E=\sum_{i}^{\text {cluster }} E_{i} .
$$


$E_{i}$ is the amplitude of the signals in energy units $(\mathrm{GeV})$, and $C_{i}$ the coefficients necessary to perform this conversion. The electromagnetic shower created by a particle in the calorimeter decreases exponentially in space. To account for this, we include the $\ln \left(E_{i} / E\right)$ term in (3.17), which increases the weight of neighboring blocks accordingly to the energy they registered. $W_{0}$ allows us to adjust the weight of each block as a function of the registered energy. When $W_{0} \rightarrow \infty$, the weight distribution is uniform, whereas for small values of $W_{0}$ the blocks with higher energy prevail. $W_{0}$ works as a threshold as well. Blocks with a relative energy below $e^{-W_{0}}$ will be assigned a $w_{i}=0$. Since the computation of the particle quadrivector uses the impact point in the calorimeter as well as the impact point of the beam in the target, we must apply a correction to account for the fact that the electromagnetic shower does not begin at the surface of the calorimeter, but at a certain depth. Additionally, we must take into account the fact that we don't have a point-like target. Finally, the corrected position is given by

$$
x_{\text {corr }}=x\left(1-\frac{a}{\sqrt{L_{h i t}^{2}+x^{2}}}\right),
$$

where $a$ is the depth at which the electromagnetic shower starts (about $7 \mathrm{~cm}$, optimized with Monte Carlo simulation and data from elastic runs), and $L_{h i t}$ is the distance from the target impact point to the calorimeter.

\subsubsection{Event time}

The goal of the waveform analysis and clustering processes is to reconstruct the energy of the leptoproduced photon from the calorimeter signals. This wouldn't be possible if the signals didn't correspond to the same events. We can calculate the time of arrival of the photon by weighting the signals composing the cluster through the expression

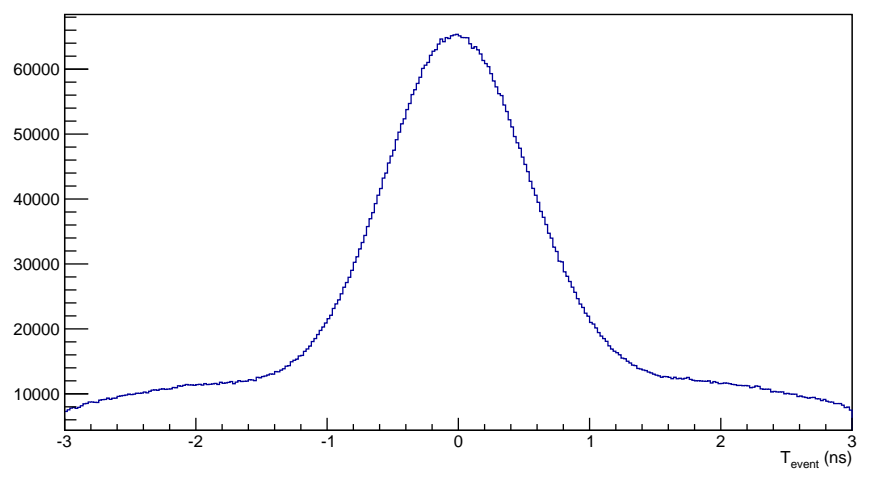

Figure 3.10: $T_{\text {event }}(n s)$ for Kin 3-high kinematic. $\sigma \sim 0.6 n s$. 


$$
T_{\text {event }}=\frac{\sum_{i}^{\text {cluster }} A^{i}\left(t^{i}-t_{\text {corr }}^{i}\right)}{\sum_{i}^{\text {cluster }} A^{i}},
$$

where $A^{i}$ are the amplitudes of the signals, $t$ is their time of arrival and $t_{\text {corr }}$ the correction in time. Due to the time corrections applied in section 3.1.5, in most of the events the photon has a $T_{\text {event }}$ between $\pm 1 n s$. We rule out events outside the $\pm 3 n s$ range, which exceeds $3 \sigma$ of the $T_{\text {event }}$ distribution, since these events do not match the trigger from the spectrometer.

\subsubsection{Clustering thresholds}

When selecting a clustering threshold a couple of issues are to be taken into account. The first one is that we will search for DVCS events in events which only formed one cluster. The reason for this is obvious, we only expect one photon per reaction to hit the calorimeter. The second one has to do with the subtraction of the background generated by $\pi^{0}$ decays. Sometimes the interaction of the electron beam with the target will result in the production of a $\pi^{0}$ that will decay into two photons. These photons may reach the calorimeter and alter our data. We must find a way to remove the background generated by this phenomenon. In order to do this, we keep the data from events that formed two

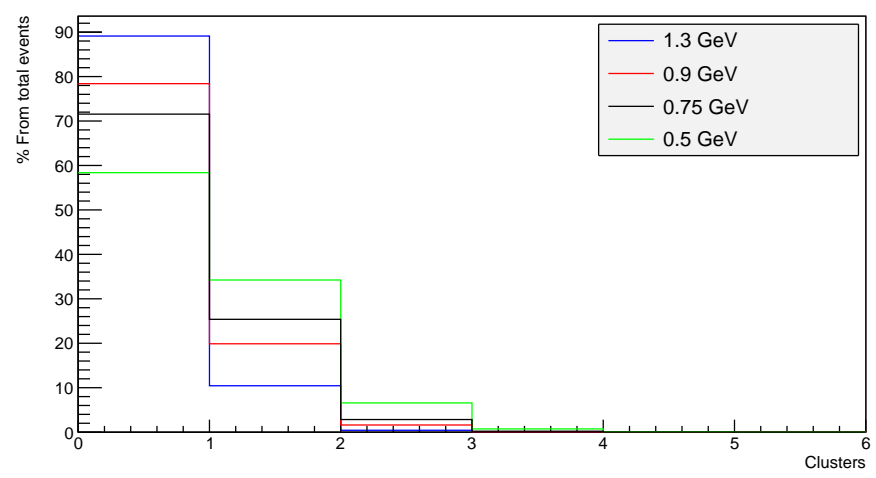

Figure 3.11: Variation of the number of clusters with clustering threshold.

clusters, where we will find data from $\pi^{0}$ decays (see chapter 4). In Figure 3.11, we observe how the number of one-cluster and two-cluster events change as the clustering threshold varies. If the clustering threshold is too high most of the events will form only one cluster, whereas if it is too low any signal will form a cluster. We look for a threshold which provides enough two-cluster events without decreasing the number of one-cluster 
events much. Also, we are interested in reducing the amount of three cluster events, since we will discard all information from this kind of events. We chose to set the threshold at $0.75 \mathrm{GeV}$ since it reduces the amount of three cluster events (less than 3\%) while keeping a good amount of one and two-cluster events.

\subsection{Calorimeter calibration}

The calorimeter calibration consists in calculating the coefficients that allow the conversion between energy channels and energy units for each block conforming the apparatus. Since calibration may vary as time goes by, several calibration sessions were conducted as the experiment went by. The main cause of changes in the calibration is the loss of block transparency due to their continuous exposure to high energy radiation. This phenomenon contributes to the degradation of the blocks response with the course of time.

\subsubsection{Calibration procedure}

For the calibration procedure, the elastic reaction $\left(e+p \rightarrow e^{\prime}+p^{\prime}\right)$ was studied, using a $\mathrm{LH}_{2}$ target. The scattered proton was detected by the high resolution spectrometer which at the same time served as a trigger for the detection of the scattered electron by the calorimeter. The goal of this configuration is to be able to compute the scattered electron energy $E^{H R S}$ using the known data from the beam energy $E_{b}$, the proton mass $M_{p}$ and the scattered proton energy $E_{p}$, and use them as a reference to fit the coefficients assigned to the signals of the calorimeter.

$$
E^{H R S}=E_{b}+M_{p}-E_{p} .
$$

To better fit the value of the calibration coefficients we define a $\chi^{2}$ that includes the relation between coefficients, signal amplitudes and electron energies through the expression

$$
\chi^{2}=\sum_{j=1}^{N}\left(\frac{E_{j}^{H R S}}{\sigma}-\sum_{i=0}^{207} \frac{C_{i} A_{j}^{i}}{\sigma}\right)^{2} .
$$

Where $A_{j}^{i}$ is the signal amplitude for each event $j$ in block $i$, and $\sigma$ is related to the energy resolution of the detection package (estimated to be around 3\%). By minimizing (3.22) as a function of the coefficients, we obtain a series of 208 linear equations that depend on $C_{i}$

$$
\frac{\chi^{2}}{\partial C_{k}}=-2 C_{k} \sum_{j=1}^{N}\left(\frac{E_{j}^{H R S}}{\sigma^{2}}-\sum_{i=0}^{207} \frac{C_{i} A_{j}^{i}}{\sigma^{2}}\right) A_{j}^{k}=0, \quad \forall k=0,1, \ldots, 207
$$




$$
\sum_{i=0}^{207}\left[\sum_{j=1}^{N} \frac{A_{j}^{k} A_{j}^{i}}{\sigma^{2}}\right] C_{i}=\sum_{j=1}^{N} \frac{E_{j}^{H R S} A_{j}^{k}}{\sigma^{2}}, \quad \forall k=0,1, \ldots, 207 .
$$

By solving this system of equations, we can obtain the coefficients for the 208 blocks. We begin by defining the $208 \times 208$ matrix $M_{i k}$ and the column vector $V_{k}(208)$,

$$
M_{i k}=\sum_{j=1}^{N} \frac{A_{j}^{k} A_{j}^{i}}{\sigma^{2}}, \quad V_{k}=\sum_{j=1}^{N} \frac{E_{j}^{H R S} A_{j}^{k}}{\sigma^{2}} .
$$

Inverting the matrix $M_{i k}$ and multiplying it by the column vector $V_{k}$ gives us the 208 coefficients. We can also calculate the coefficients error $\delta\left(C_{i}\right)$ ),

$$
\begin{gathered}
C_{i}=M_{i k}^{-1} V_{k}, \quad \forall k=0,1, \ldots, 207 . \\
\delta\left(C_{i}\right)=\sqrt{\left(M_{i i}^{-1}\right) .}
\end{gathered}
$$

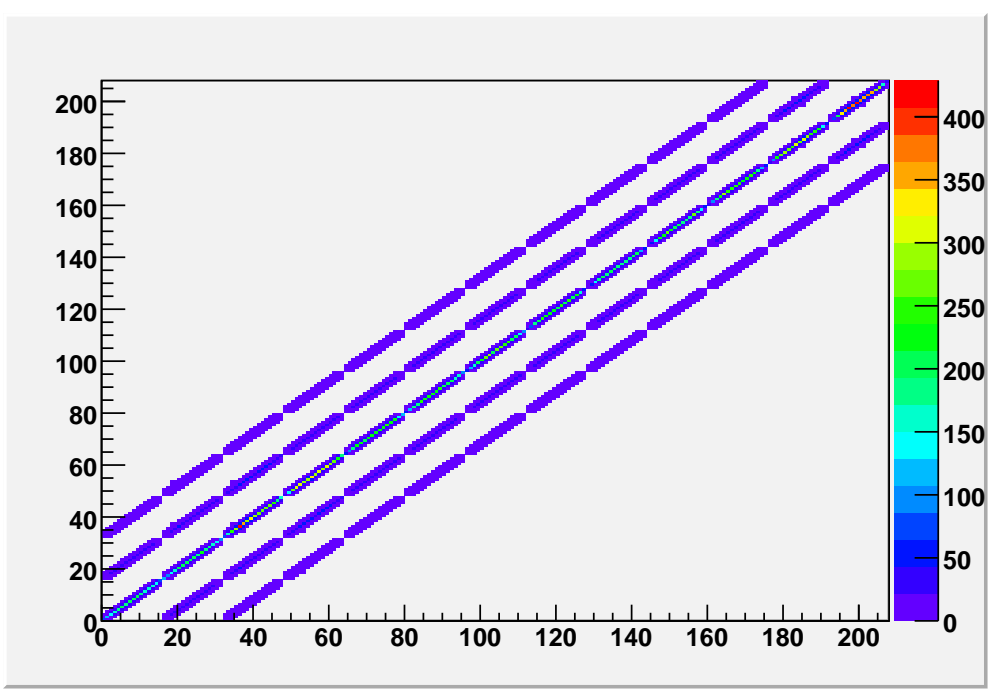

Figure 3.12: Matrix $M_{i k}$. Each axis contains the blocks numbering. The central line corresponds to $A_{j}^{k} A_{j}^{i}$ with $\mathrm{i}=\mathrm{k}$ (same block), and the rest to $A_{j}^{k} A_{j}^{i}$ for the neighboring blocks. The discontinuities in the lines appear when blocks in the edges of the calorimeter are involved (they have less neighboring blocks). 


\section{Elastic events, selection in the HRS}

It is possible to identify elastic scattering reactions by using the correlation between momentum and scattering angle for particles detected by the spectrometer. For elastic reactions we expect a linear correlation between these two variables, which will help us select the processes needed for the calibration. We obtained the deviation of the scattered particles momentum from the HRS central momentum as well as their deviation in $\mathrm{mrad}$, and visualized these variables (the HRS provides this information in a percentual way).

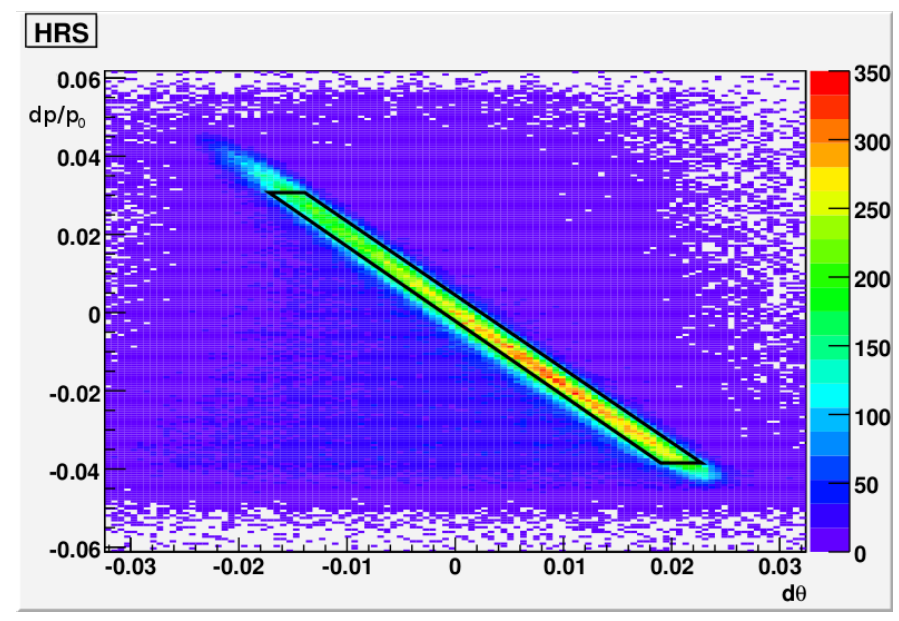

Figure 3.13: Deviation of momentum and angle from HRS central values (parts per one). The interior of black frame corresponds to the selected events employed in the calibration.

In Figure 3.13, we see a background of many kinds of detected particles. The cut tries to select most of the events in which we observe the correlation between momentum and angle, and discard those not coming from the elastic reaction $\left(e+p \rightarrow e^{\prime}+p^{\prime}\right)$.

\subsubsection{Calorimeter resolution}

In the calorimeter, the amplitude of the signal pulse is proportional to the energy deposited by the incident radiation. This creates an electromagnetic shower of Čerenkov photons that fluctuates in number from one detection to another. For a detected energy of 1 $\mathrm{GeV}$ about $1000 \pm 32$ Čerenkov photons are emitted (estimation obtained from a Monte Carlo simulation). This fluctuation establishes how accurately the calorimeter is able to distinguish peaks of similar energy. We also check the angular resolution, which refers to how well the calorimeter can distinguish the hitting point on its surface between two impacts which are separated by a small angular distance. 


\section{Energy resolution}

It is possible to compute the calorimeter resolution using the elastic events from the calibration sessions, but we will not take into account events with a hitting point in the edge blocks (see Figure 3.14). In these cases not all the energy is deposited in the calorimeter, and therefore, the resolution of the calorimeter on the edges is much worse. This makes it impossible to have an acceptable value to compare with the data from the spectrometer.

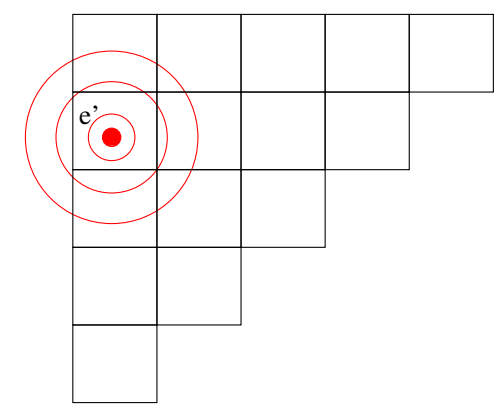

Figure 3.14: Impact of $e^{\prime}$ in the left upper corner of the calorimeter.

In this calculation, we are only concerned with the blocks that are completely surrounded by other blocks. Once the calibration coefficients are known, we compare the value of the energy $E^{H R S}$, obtained using (3.21), and the reconstructed energy $E^{C a l}$, obtained using the coefficients.

$$
E^{H R S}-E^{C a l}=\Delta E \quad \text { where } \quad E^{C a l}=\sum_{i}^{\text {cluster }} C_{i} A^{i} .
$$

For an ideal detector $\Delta E_{j}=0$ for every event $j$, but for our detector we obtain a Gaussian distribution around 0 (Figure 3.15), that will allow us to know its energy resolution. This resolution comes from dividing the $\sigma$ of the Gaussian distribution by the mean energy of the scattered electron $E^{C a l}(M e a n)$. The mean value of the energy of the scattered electron is obtained from fitting the distribution of $E^{\text {Cal }}$ (see Figure 3.16).

$$
\text { Energy resolution } \equiv \frac{\sigma(\Delta E)}{E^{C a l}(\text { Mean })}
$$

The distributions shown above correspond to the Elastic 2 (18 Nov) calibration session. For each calibration setup the following table shows the values of the resolution for both 


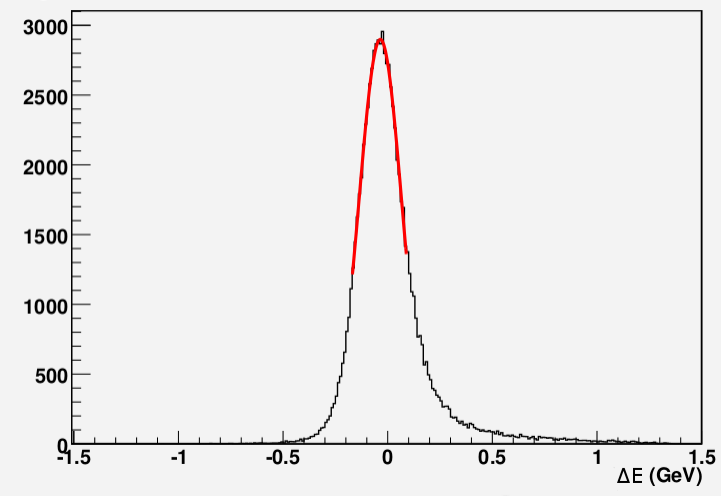

Figure 3.15: $\Delta E . \sigma\left(\Delta E_{j}\right)=0.098 \mathrm{GeV}$

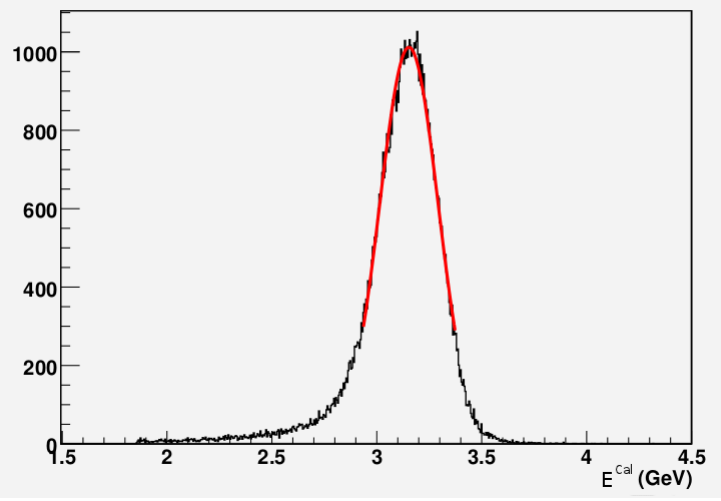

Figure 3.16: $E^{C a l} . E^{C a l}($ Mean $)=3.15 \mathrm{GeV}$

sessions of each setup (three for the "Elastic 1" setup). The sessions are arranged chronologically.

\begin{tabular}{c|c|c|c|c} 
Date & $E_{b}(\mathrm{GeV})$ & $E^{\mathrm{Cal}}(\mathrm{M})(\mathrm{GeV})$ & $\sigma\left(\Delta E_{j}\right)(\mathrm{GeV})$ & Resolution \\
\hline Elastic 1 (17 Oct) & 5.552 & 3.79 & 0.123 & $3.25 \%$ \\
Elastic 1 (19 Oct) & 5.552 & 3.86 & 0.159 & $4.12 \%$ \\
Elastic 2 (25 Oct) & 4.454 & 3.18 & 0.097 & $3.05 \%$ \\
Elastic 2 (26 Oct) & 4.454 & 3.16 & 0.097 & $3.07 \%$ \\
Elastic 2 (17 Nov) & 4.454 & 3.16 & 0.100 & $3.16 \%$ \\
Elastic 2 (18 Nov) & 4.454 & 3.15 & 0.098 & $3.11 \%$ \\
Elastic 1 (14 Dec) & 5.552 & 3.85 & 0.108 & $2.81 \%$
\end{tabular}

Table 3.3: Energy resolutions for each calibration session.

There were several problems concerning the two first calibration sessions, addressed later in this chapter. We observe that when the beam energy $E_{b}$ is increased, we have a better resolution. This is due to the inverse dependence of the energy resolution with $E^{C a l}($ Mean $)$. 


\section{Angular resolution}

Since we can trace back the impact point of the electron in the target and the impact point of the scattered electron in the calorimeter surface we can calculate the scattering angles $\theta$ and $\phi$, defined as [58]

$$
\begin{aligned}
\operatorname{tg}(\theta) & =\frac{p_{x}}{p_{z}} \\
\operatorname{tg}(\phi) & =\frac{p_{y}}{\sqrt{p_{x}^{2}+p_{z}^{2}}} .
\end{aligned}
$$

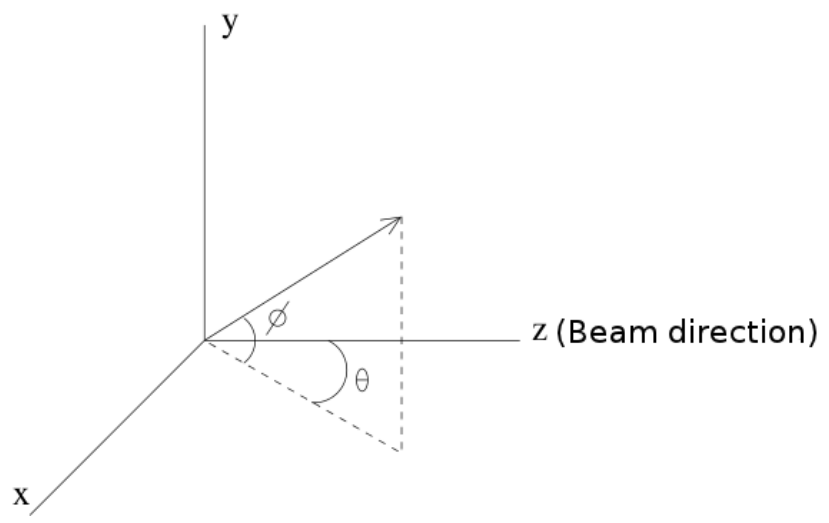

Figure 3.17: Coordinate system

In order to obtain these angles, we study the kinematics of the elastic scattering $(e+p \rightarrow$ $\left.e^{\prime}+p^{\prime}\right)$. The momentum conservation equation reads

$$
\begin{aligned}
\left(0,0, p_{1 z}\right)_{e}+(0,0,0)_{p} & =\left(p_{1 x}^{\prime}, p_{1 y}^{\prime}, p_{1 z}^{\prime}\right)_{e^{\prime}}+\left(p_{2 x}^{\prime}, p_{2 y}^{\prime}, p_{2 z}^{\prime}\right)_{p^{\prime}} \\
\left(p_{1 x}^{\prime}, p_{1 y}^{\prime}, p_{1 z}^{\prime}\right) & =\left(-p_{2 x}^{\prime},-p_{2 y}^{\prime}, E_{b}-p_{2 z}^{\prime}\right)
\end{aligned}
$$

since $p_{1 z}=E_{b}$.

Now we write the scattering angles in terms of the electron momentum given by the calorimeter $\left(\theta_{e^{\prime}}, \phi_{e^{\prime}}\right)$, and the momentum from the scattered proton and the beam energy $\left(\theta_{e^{\prime}}^{\prime}, \phi_{e^{\prime}}^{\prime}\right)$, so we can compare both results.

$$
\begin{gathered}
\theta_{e^{\prime}}=\operatorname{atan}\left(\frac{p_{1 x}^{\prime}}{p_{1 z}^{\prime}}\right), \quad \theta_{e^{\prime}}^{\prime}=\operatorname{atan}\left(\frac{-p_{2 x}^{\prime}}{E_{b}-p_{2 z}^{\prime}}\right) . \\
\phi_{e^{\prime}}=\operatorname{atan}\left(\frac{p_{1 y}^{\prime}}{\sqrt{\left(p_{1 x}^{\prime}\right)^{2}+\left(p_{1 z}^{\prime}\right)^{2}}}\right), \quad \phi_{e^{\prime}}^{\prime}=\operatorname{atan}\left(\frac{p_{2 y}^{\prime}}{\sqrt{\left(p_{2 x}^{\prime}\right)^{2}+\left(E_{b}-p_{2 z}^{\prime}\right)^{2}}}\right) .
\end{gathered}
$$

The difference between the scattering angles obtained from the HRS and the calorimeter are related to the angular resolution of the calorimeter,

$$
\Delta \theta=\theta_{e^{\prime}}^{\prime}-\theta_{e^{\prime}}, \quad \Delta \phi=\phi_{e^{\prime}}^{\prime}-\phi_{e^{\prime}}
$$


Again, as we did with the scattered electron energy, we fit the distribution of the difference measures from the HRS and the calorimeter. Figures 3.18 and 3.19 show the distributions for these differences for both angles, $\theta$ and $\phi$, for the calibration session Elastic 2 (18 Nov). We take the $\sigma$ of the amplitudes as the uncertainty of each angle in radians.

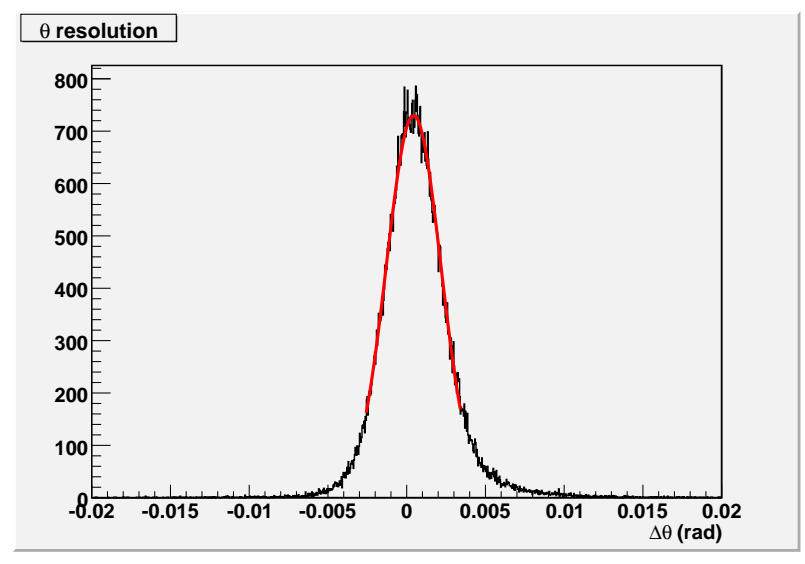

Figure 3.18: $\Delta \theta . \sigma(\Delta \theta)=1.75 \mathrm{mrad}$.

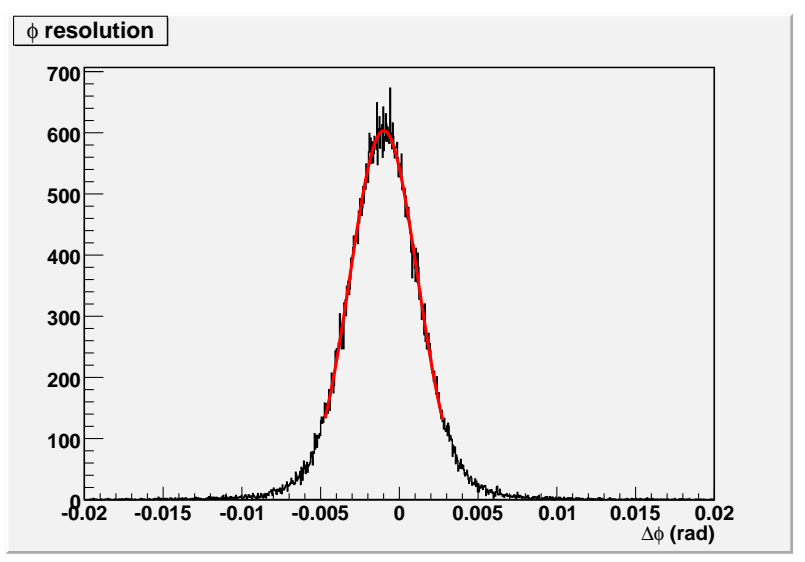

Figure 3.19: $\Delta \phi . \sigma(\Delta \phi)=2.14$ mrad.

Since the distance of the surface of the calorimeter from the target is $5.5 \mathrm{~m}$, we can convert the uncertainty in the hitting point location from angular units to a distance on the surface. We multiply $\sigma(\Delta \theta)$ and $\sigma(\Delta \phi)$ by this factor to find the uncertainties on the surface $\Delta x$ and $\Delta y$, respectively.

Table 3.4 shows the angular resolution results for every calibration session

\begin{tabular}{c|c|c|c|c} 
Date & $\sigma(\Delta \theta)(\mathrm{mrad})$ & $\sigma(\Delta \phi)(\mathrm{mrad})$ & $\Delta x(\mathrm{~cm})$ & $\Delta y(\mathrm{~cm})$ \\
\hline Elastic 1 (17 Oct) & 1.54 & 1.91 & 0.85 & 1.05 \\
Elastic 1 (19 Oct) & 1.46 & 1.98 & 0.80 & 1.09 \\
Elastic 2 (25 Oct) & 1.71 & 2.20 & 0.94 & 1.21 \\
Elastic 2 (26 Oct) & 1.69 & 2.20 & 0.93 & 1.21 \\
Elastic 2 (17 Nov) & 1.76 & 2.15 & 0.97 & 1.18 \\
Elastic 2 (18 Nov) & 1.75 & 2.14 & 0.96 & 1.18 \\
Elastic 1 (14 Dec) & 1.96 & 1.94 & 1.08 & 1.07
\end{tabular}

Table 3.4: Angular resolutions. 


\section{$\pi^{0}$ decay test}

We can carry on a test to check the calibration of the calorimeter by selecting events where $\pi^{0} \rightarrow \gamma+\gamma$. Using the information from the experimental data for the energy of the photons from two-cluster events we calculate the $\pi^{0}$ mass:

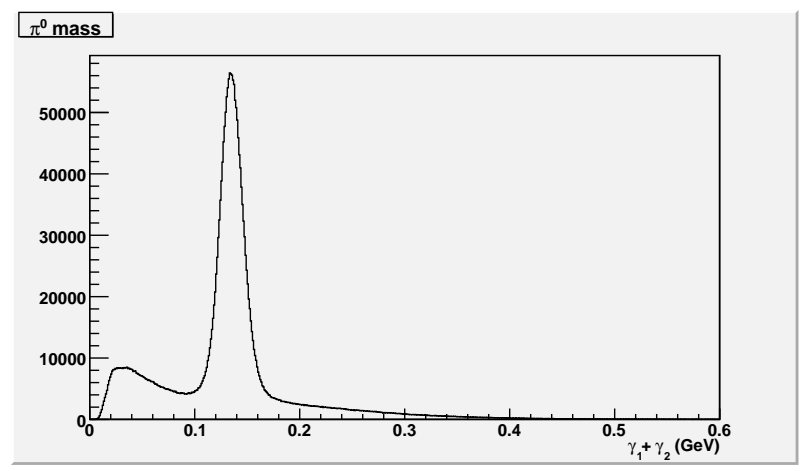

Figure 3.20: Invariant mass. 2 cluster events.

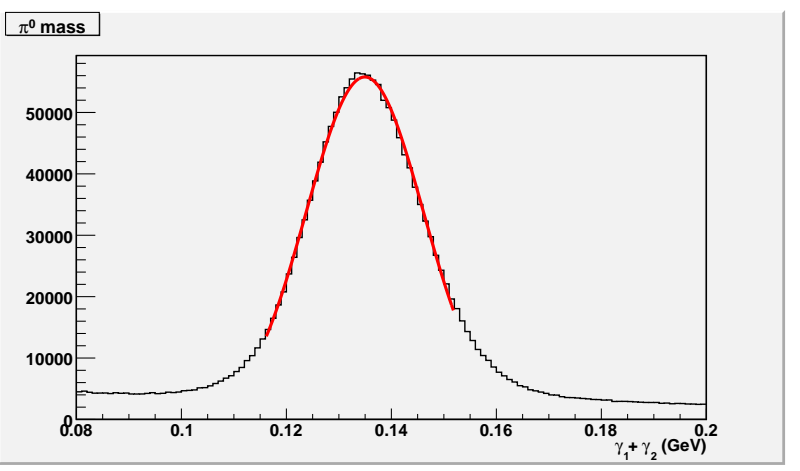

Figure 3.21: Invariant mass, $M_{\pi^{0}}$ fit.

$$
M_{\pi^{0}}: 134.95 \pm 0.12 \mathrm{MeV}
$$

Theoretical value: $134.9766(6) \mathrm{MeV}$

Figure 3.21 is a zoom of the peak of the invariant mass, shown on Figure 3.20, so the fit can be appreciated in detail. This value for $M_{\pi^{0}}$ was obtained by using all the experimental data, and it is in agreement with the theoretical value.

\subsubsection{Calibration problems}

Some of the problems concerning block malfunctioning are stated in the next paragraphs.

Signals from blocks 73 and 77 had been swapped during all the experiment. To solve this we had to undo the swap during the data analysis process.

During calibration session Elastic 1 (17 Oct) block 119 wasn’t working properly. All data stored from this block were found to be electronic noise. The same problem occurred during calibration session Elastic 1 (19 Oct), this time concerning blocks 119, 163, 167, 177 and 181. The lack of information on the particles energy rends impossible the task of calculating the calibration coefficients for these blocks. We assigned a symbolic value of 1 to all of them. Moreover, this problem also affects the calculation of the coefficients of the neighboring blocks, since part of the particles energy is missing. Since most of the experiment runs took place after calibrating session Elastic 2 (25 Oct) the impact of this issue on the data is minimal. 
From calibration session Elastic 2 (17 Nov) until the end of the experiment, signals from block 89 show a reflexion of the signal within the cable that transmits the data from the photomultipliers. Fits of these signals are less accurate than the rest.

During calibration session Elastic 1 (14 Dec) (last calibration session), the HRS trigger wasn't working as expected. As explained in chapter $2, t=0$ is supposed to be given by the scintillator plane S2, but during this calibration, for many events, it was given by the Cerenkov detector. This complicated the process of time correction of the pulses, forcing us to use two different corrections depending on which detector set $t=0$.

\subsubsection{Coefficients comparison}

We compare the coefficients obtained through the different measurement sessions to prove their consistency [59]. At the same time, we can observe the process of loss of transparency of the blocks due to their exposure to radiation. The loss of transparency affects the coefficients in the following way: since the response of the block decreases over time, the coefficients increase to account for this phenomenon. Therefore, if we were to divide the coefficients of one calibration session by those of a latter one, we should obtain a result higher than 1 for each block. However, the voltages of the PMTs have different settings for some of the sessions. Due to this, the absolute value of the coefficients is not always directly comparable, so we resort to the expression for the gain, $G$, of the PMTs

$$
G=\alpha V^{\beta} .
$$

Where $\alpha$ is a constant, $\mathrm{V}$ is the value of the PMTs voltage $( \pm 1 V)$, and $\beta$ has a typical value of $\sim 7.0 \pm 0.1$ for the calorimeter PMTs. Using (3.36), the coefficients $C_{1}$ and $C_{2}$ of two different calibration sessions now read

$$
\frac{1}{C_{1}}=\alpha V_{1}^{\beta}, \quad \frac{1}{C_{2}}=\alpha V_{2}^{\beta},
$$

and therefore

$$
\frac{C_{2}}{C_{1}}=\left(\frac{V_{1}}{V_{2}}\right)^{\beta} \longrightarrow 1=\frac{C_{1}}{C_{2}}\left(\frac{V_{1}}{V_{2}}\right)^{\beta} .
$$

Below, we can observe the comparison between the coefficients of each calibration session and the coefficients from the next session (arranged chronologically). The comparison for the blocks located in the borders of the calorimeter is in red to point out that due to their position on the detector they are less reliable than the others. When there is a change in the voltages, it will be indicated by ( $\mathrm{VCh}$ ). The statistic error of the coefficients is very small, $\sim 0.08 \%$, so we neglect it. 


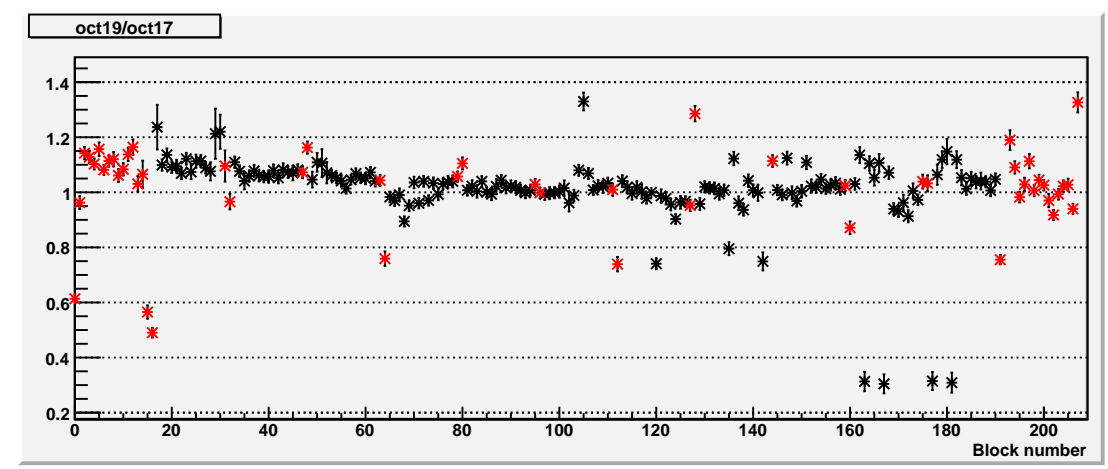

Figure 3.22: Ratio between the coefficients obtained in calibration session 19 Oct and 17 Oct. The ratio for blocks located in the edges of the calorimeter is shown in red. (VCh).

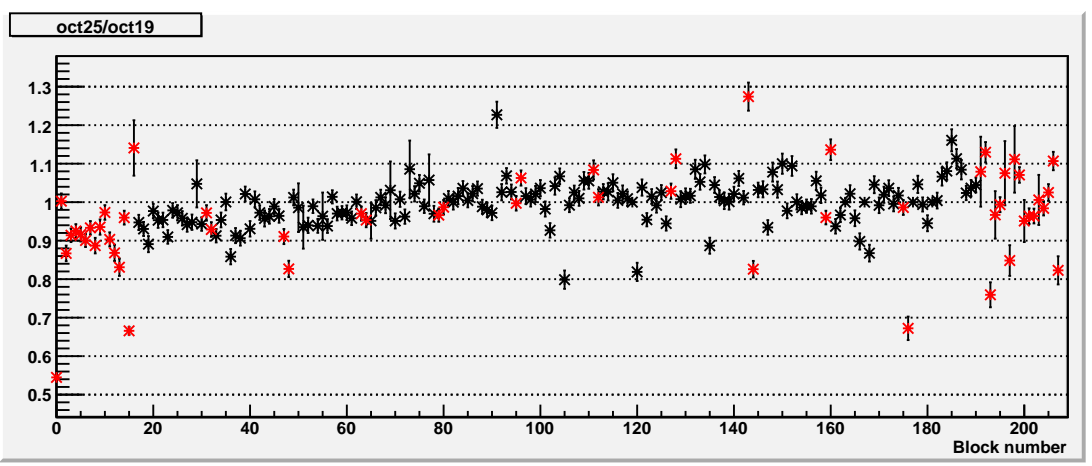

Figure 3.23: Ratio between the coefficients obtained in calibration session 25 Oct and 19 Oct. The ratio for blocks located in the edges of the calorimeter is shown in red. (VCh).

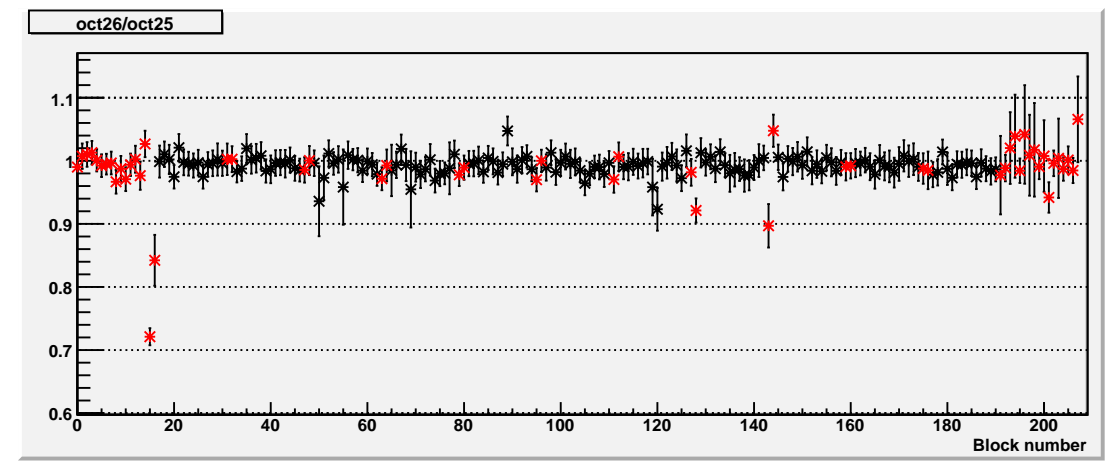

Figure 3.24: Ratio between the coefficients obtained in calibration session 26 Oct and 25 Oct. The ratio for blocks located in the edges of the calorimeter is shown in red. (VCh). 


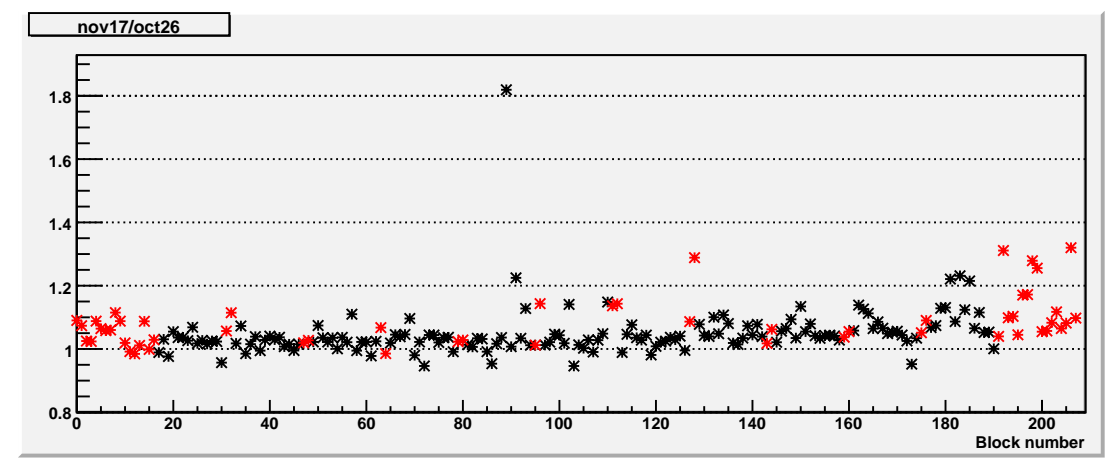

Figure 3.25: Ratio between the coefficients obtained in calibration session 17 Nov and 26 Oct. The ratio for blocks located in the edges of the calorimeter is shown in red.

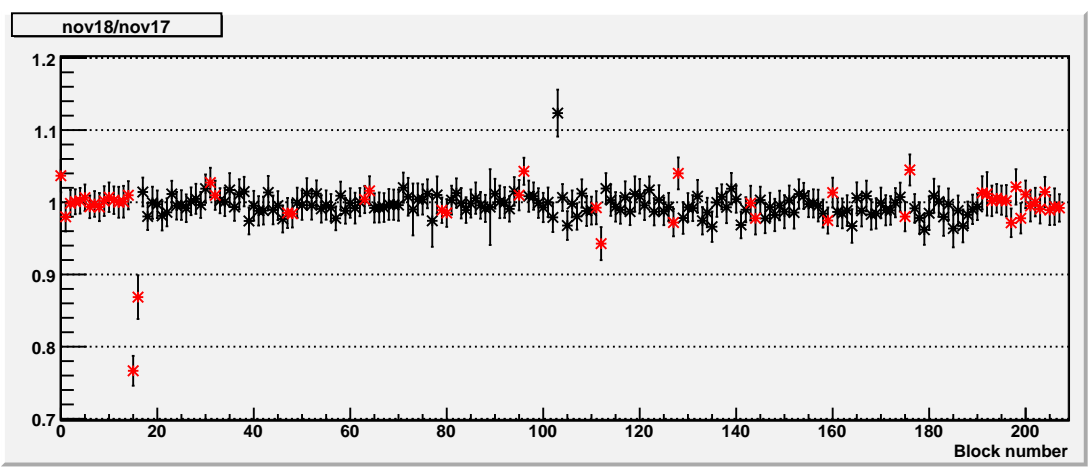

Figure 3.26: Ratio between the coefficients obtained in calibration session 18 Nov and 17 Nov. The ratio for blocks located in the edges of the calorimeter is shown in red. (VCh).

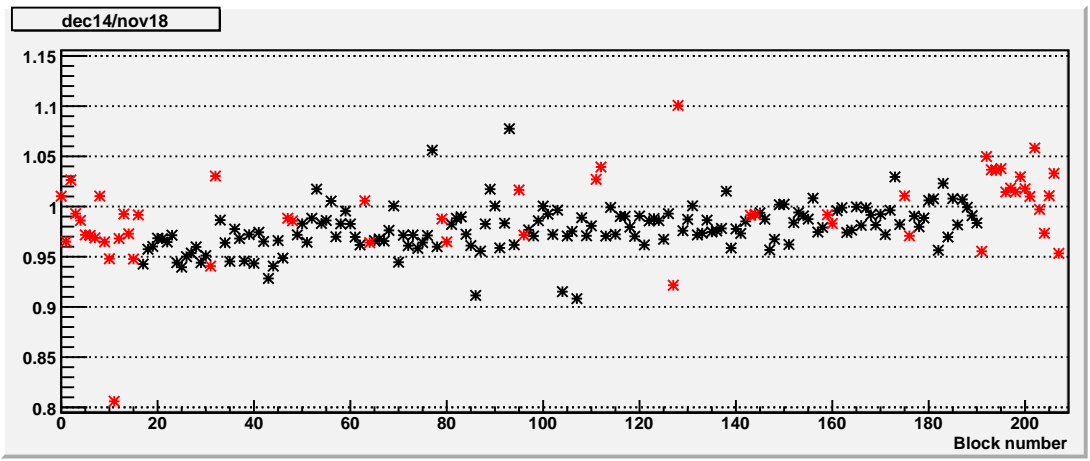

Figure 3.27: Ratio between the coefficients obtained in calibration session 14 Dec and 18 Nov. The ratio for blocks located in the edges of the calorimeter is shown in red. 
For the calibrations Elastic 2 (25 Oct) and onwards (the ones employed for data analysis, without the issues of having disconnected blocks), we observe a trend of higher transparency loss for the blocks located on the left of the surface of the calorimeter (higher block number), whenever there is a big lapse of time (17 Nov/ 26 Oct and 14 Dec/ 18 Nov). This is consistent with the fact that these blocks are nearer to the electron beam than the rest. For the case of the two last calibration sessions, (14 Dec/ 18 Nov), it appears as if most of the blocks had gained transparency, contrary to what one might have expected. This is due to an increase in the gain , $G$, of the PMTs during that period, which resulted in the overall decrease of the coefficients for the calibration session of 14 Dec [60]. The voltage of each PMT is adjusted in order to keep a constant gain during the whole experiment. Figure 3.28 shows the variation in the PMTs voltage needed to maintain the gain at a constant value, and compares it to a previous JLab experiment focused on DVCS [61]. The fact that the voltage had to be decreased, as shown in the figure, indicates that the PMTs were working at a higher gain value that the one expected.

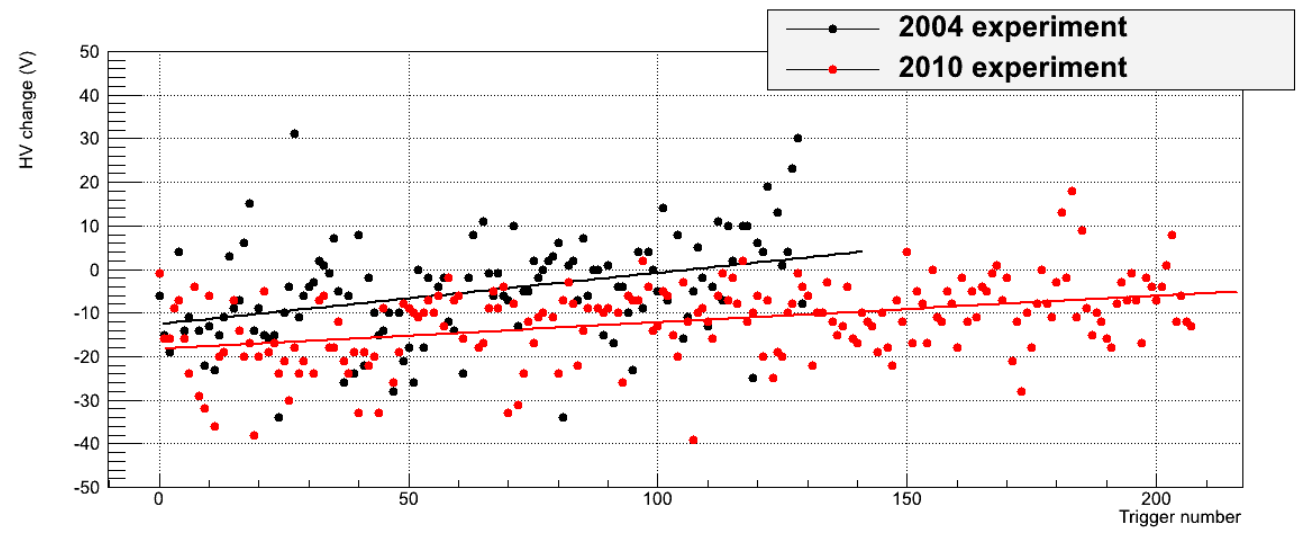

Figure 3.28: Variations observed during the first experiment (black) and those observed during the second experiment (red), relative to the beginning of each experiment. Notice that the same PMT was not in the same trigger number (horizontal axis) for both 2004 and 2010 experiment.

For the cases where there is almost no lapse of time between calibrations (26 Oct/ 25 Oct) and (18 Nov/ $17 \mathrm{Nov})$, we see that for most of the blocks the coefficients are stable. 

$\bar{c}_{\text {comen }} 4$

\section{Background subtraction and extraction of observables}

Contributions from different sources besides DVCS reactions alter our data and must be removed. Most of the undesired detections are discarded by applying cuts in kinematic variables. These variables are related to the detectors geometry, like for example: the acceptance of the HRS or the target impact point. But not all the removal can be done with cuts, the subtraction of the $\pi^{0}$ electroproduction contribution must be tackled in some other way, as well as the subtraction of accidental detections. Finally, radiative corrections are taken into account, as well as the inclusion of DVCS events found in multicluster events. Once all cuts, background removal and other corrections have been applied, we will be left with the final data, which we will employ in cross section computations. These computations are done with the help of a Monte Carlo simulation, which main characteristics are shown in this chapter.

At the end of the chapter, the different cross sections for the photon leptoproduction process as well as the comparison between data and Monte Carlo simulation, for every studied kinematics, are shown.

\subsection{Events selection}

During the data acquisition, the calorimeter registers the energy of particles coming from different kinds of events. In order to minimize the impact of the detections not coming from DVCS reactions, we make a selection of events. This selection is made applying different cuts to some kinematic variables related to the target, the HRS and the calorimeter. 


\subsubsection{Target, impact point}

Due to the aluminum cylindrical target cells in which the target is placed, not all scattered electrons come from the $\mathrm{LH}_{2}$ or $\mathrm{LD}_{2}$ materials. Since we can reconstruct the impact point of the beam on the target, using the data from the HRS, it is possible to check whether or not there was an interaction with the desired target.

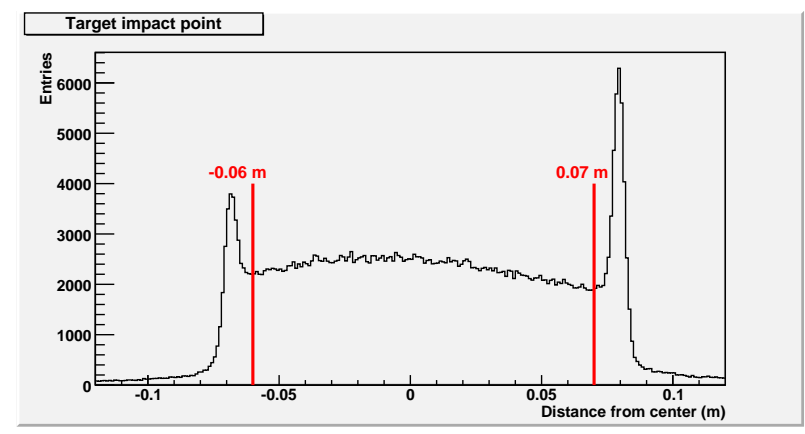

Figure 4.1: Target impact point. $-0.06 m<$ Selected events $<0.07 m$.

Figure 4.1 shows the impact point with the target along the beam line. The peaks in both sides of the histogram come from impacts on the aluminum cell, rich in protons. To avoid taking these interactions as data we impose a cut on the target between $-0.06 \mathrm{~m}$ and $0.07 \mathrm{~m}$.

\subsubsection{HRS efficiency}

We saw in chapter 2 that one of the main components of the HRS is a Čerenkov detector. This detector is employed for electron identification, and, with the scintillator plane $\mathrm{S}_{2}$, it serves as trigger for the HRS. It is important to keep track of the Čerenkov detector's efficiency in electron identification because a low efficiency would result in missing too many DVCS events. A set of runs (efficiency runs) were taken with this purpose. However, the trigger system for those runs did not include the Cerenkov detector, since it is not possible to study the efficiency of a detector if it is serving as trigger. Instead, the scintillator planes S1 and S2 were used as trigger for the efficiency runs.

When measuring the efficiency of the detector we want to be sure that the particles arriving at the device are electrons. Pions are an important source of background for this matter. In order to discriminate pions from electrons, an electromagnetic calorimeter (pion rejector) was employed. Electrons arriving at the pion rejector deposit more energy than pions, since electrons create purely electromagnetic showers, so a cut in energy was set in order to select the electrons [62]. However, specially at low momentum, pions have a small probability to create hadronic showers and thus lose all of their energy. To evaluate this effect, the pion rejector spectrum is fitted by the sum of a Gaussian distribution 
with a radiative tail $\mathrm{S}(\mathrm{E})$ for the electrons and a second order polynomial for the pions. The proportion of electrons detected by the Čerenkov detector from the total of the pion rejector electron detections is known as the efficiency of the Črenkov detector. The results of the study show a constant efficiency of $98.5 \%$ from run 8302 until the end of the experiment. Before run 8302 the efficiency was $95 \%$ due to a higher energy threshold set on the Cerenkov detector. This only affected a part of the Kin 3-low kinematics, and therefore, in overall, the results for the efficiency are satisfactory.

The efficiency of the scintillator planes S1 and S2 was studied as well. For this purpose, two types of events were taken into account: the ones with a detection from the Cherenkov and S1 and the ones with a detection from the Čherenkov and S2. The efficiency was computed by comparing the response of S1 to the response of S2, with the appropriate event type. A more detailed explanation of the process can be found in [63]. S1 is composed of six paddles with two PMTs in each side (left and right), while S2 is composed of sixteen paddles with also two PMTs in each side. We show the efficiency of each set of paddles (left, right) for every efficiency run in Figure 4.2, where we observe that the four sets have about $99 \%$ efficiency.
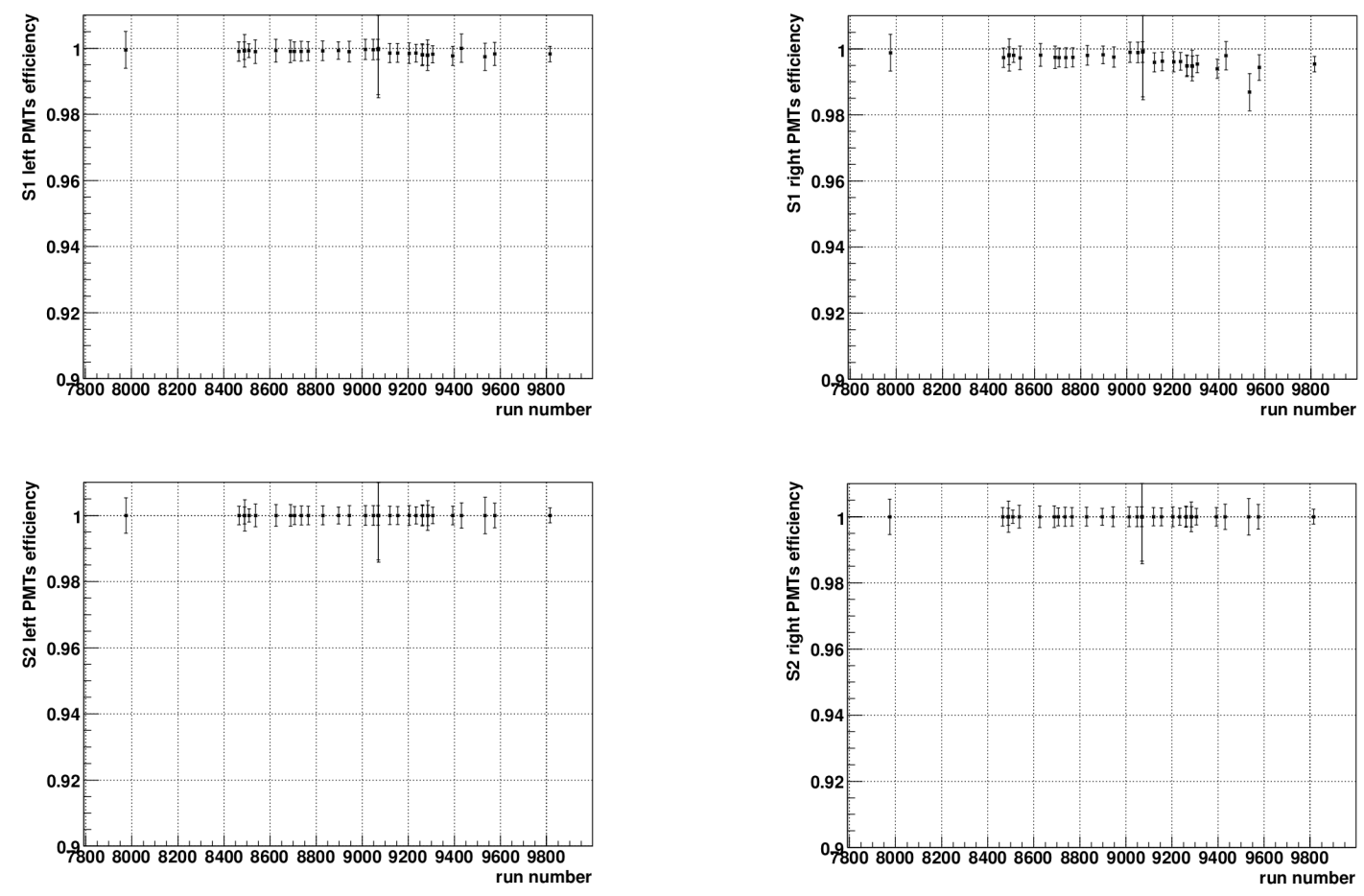

Figure 4.2: Left: Efficiency for S1 (top) and S2 (low) left PMTs. Right: Efficiency for S1 (top) and S2 (low) right PMTs. 


\subsubsection{HRS acceptance}

The trajectory of particles detected by the spectrometer is determined by its magnetic field. For every initial line of flight and momentum of the particle at the spectrometer entrance, there is a definite trajectory of the particle, which results in either the particle passing through the spectrometer to its focal plane, or getting absorbed by internal spectrometer apertures. The region of solid angle where particles, for a certain range of momentum, can be detected is called the acceptance of the detector. For purposes of cross-section extraction, software cuts are usually placed on the reconstructed target variables to select a region inside the "good acceptance" region. Placing the cuts so that they define a large region but still lying within the "good acceptance" region allows to include more particles in the cross-section analysis.

In order to compute the acceptance of the HRS into the analysis of cross sections, a Monte Carlo simulation is employed [64], so the cut applied in the acceptance must be the same for both, data and simulation. However, the acceptance region is a rather complicated region described by 4 different geometric variables. This is the reason why the cut is applied by defining a R-function. R-functions are real-valued functions that allow the use of equations that serve as boundaries of geometrical objects. We are able to construct functions that are equal to 0 on the boundary of the objects, and have different signs inside and outside the objects. In addition, the absolute value of an R-function is related to the distance to the boundaries of the geometrical object. The four variables that define the acceptance region read

$$
\begin{aligned}
y_{e^{\prime}} & =- \text { vertex } \times k_{x}^{\prime} / \sqrt{k_{x}^{\prime 2}+k_{z}^{\prime 2}}, \\
\theta_{e^{\prime}} & =\operatorname{atan}\left(k_{y}^{\prime} / \sqrt{k_{x}^{\prime 2}+k_{z}^{\prime 2}}\right), \\
\phi_{e^{\prime}} & =\operatorname{atan}\left(k_{x}^{\prime} / k_{z}^{\prime}\right)-\phi_{H R S}, \\
d p & =\frac{\sqrt{k_{x}^{\prime 2}+k_{y}^{\prime 2}+k_{z}^{\prime 2}}-p_{\text {central }}}{p_{\text {central }},}
\end{aligned}
$$

where $y_{e^{\prime}}$ is the displacement, in the transverse plane, of the trajectory relative to the HRS reference trajectory, $\theta_{e^{\prime}}$ is the vertical angle taking a positive value when pointing towards the floor and $\phi_{e^{\prime}}$ is the horizontal angle in the spectrometer frame. The Rfunction employed for the HRS acceptance has been calculated in previous experiments [65]. Applying a positive cut on the value of the HRS R-function, $r_{v a l}$, will diminish the acceptance region approximately uniformly across all its boundaries, while cutting on negative values of $r_{v a l}$ will increase the acceptance region. The value of this cut is

$$
r_{v a l}>0.005 .
$$


Sometimes the HRS detects more than one particle at a time. The VDC system and the tracking algorithm are not suited to reconstruct correctly the trajectories for these events. As we cannot perform any acceptance cut on these events, we remove them from the analysis. A correction must by applied in order to account for this removal of detections. In order to apply the correction we can make use of the data stored by the pion rejector. We can observe the energy deposit in the pion rejector of the multitrack detections to get some information about the composition of these events.

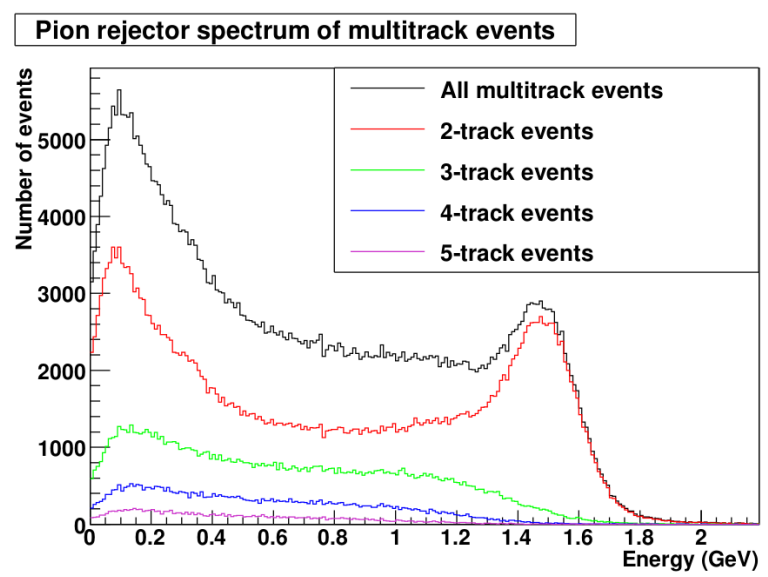

Figure 4.3: Energy deposit in the pion rejector according to the number of tracks.

As we observe in Figure 4.3, only 2-track events show a clear electron peak. The rest of multitrack events are mainly low energy events, which indicates that they are more likely pions creating secondaries particles in the VDC. Therefore, we dismiss the multitrack events with more than two tracks and express the multitrack correction as

$$
\eta_{\text {Multi }}=1+\frac{N_{2}}{N_{1}},
$$

where $N_{1}$ and $N_{2}$ stand for the amount of 1-track and 2-track events. Only those events with an deposited energy above $E_{\min }>1 \mathrm{GeV}$ are included inn the computation. This threshold depends on the HRS central momentum.

During the experiment, the HRS collimator was mislocated, obstructing the path of the scattered electrons. We can appreciate this phenomenon by comparing the data from experiment, after applying the acceptance cut, with the Monte Carlo simulation [66]. We observe, from the figure on the left in Fig.4.4, that a band is missing from the data in the lower part of the collimator plane (defined by $x_{c o l}$ and $y_{c o l}$ ). This was due to the collimator mislocation. We were able to correct this issue by applying a cut inside the real collimator plane, 


$$
\begin{aligned}
-0.0275 m & <x_{c o l}<0.0275 m \\
-0.05 m & <y_{c o l}<0.055 m .
\end{aligned}
$$
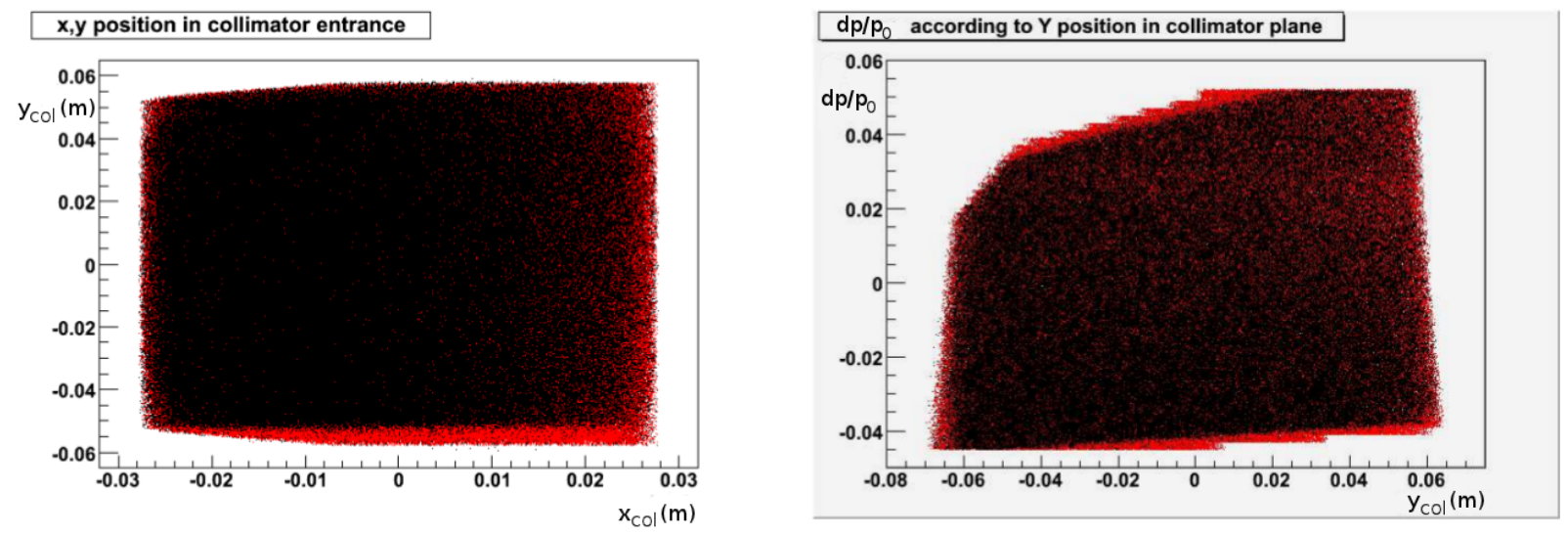

Figure 4.4: Left: comparison between the location of the collimator according to the Monte Carlo simulation (red) and according to the data (black). Right: comparison between the Monte Carlo simulation and the data (correlation between $y_{c o l}$ and the deviation of the particles momentum from the central momentum of the HRS).

In addition to this problem, a small error in the R-function was detected. This can be visualized in figure 4.4 (right), where we observe the missing bands in the data when studying the correlation between $y_{c o l}$ and the deviation of the particles momentum from the central momentum of the HRS. For this matter it was necessary to add a cut between $\mathrm{dp} / \mathrm{p}$ and $y_{c o l}$ :

$$
\frac{0.009}{0.115}\left(y_{c o l}+0.055\right)-0.045<\frac{d p}{p}<\frac{0.02}{0.08}\left(y_{c o l}+0.05\right)+0.032
$$

\subsubsection{Calorimeter, geometrical cut}

The geometrical cut in the calorimeter addresses the problem of the poor energy reconstruction in the blocks located in the borders of the calorimeter. To avoid processing data from these events, any photon impacting on any of the blocks of the borders is discarded. The cut is applied on the variables $x$ and $y$, that define the plane on the surface of the calorimeter.

$$
\begin{aligned}
& -21 \mathrm{~cm}<x<12 \mathrm{~cm}, \\
& -21 \mathrm{~cm}<y<21 \mathrm{~cm} .
\end{aligned}
$$


The cut can be visualized in figure 4.5. It selects all the particles with an impact point inside the red frame. The whole surface of the calorimeter is indicated by the blue frame, while the black region corresponds to all the registered impact points for one kinematic. We can observe how the region between the red and the blue lines (which corresponds to the blocks in the borders of the calorimeter) is only partially covered with impacts. As explained in section 3.2, the impact point is calculated by weighting the deposited energy in all the blocks in a cluster. The fact that the blocks in the border are not fully surrounded by other blocks makes it impossible for the impact point computation to yield an impact point in the border regions.

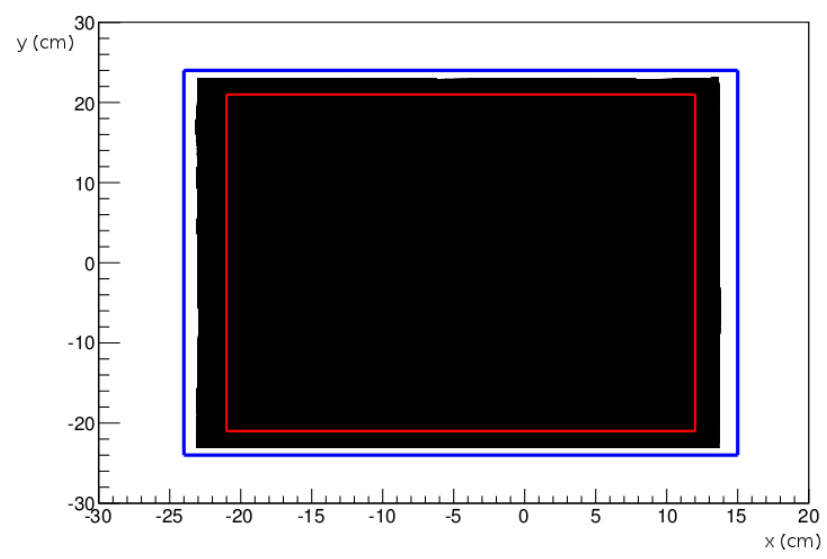

Figure 4.5: (Black) Impact points in the calorimeter surface for all Kin 3-high detections. (Red) Geometrical cut in the calorimeter surface. (Blue) Real surface of the calorimeter.

\subsection{Background subtraction}

Besides applying cuts to some variables, it is necessary to use different techniques to remove background detections. For this purpose, $\pi^{0}$ and accidental detections were studied.

\subsection{1 $\pi^{0}$ subtraction}

The production of a $\pi^{0}$, as a result of the interaction of the electron beam with the target, is a reaction that takes place during the experiment and it is a cause of background detections. The emission of a gluon, and its fragmentation into a quark and an anti-quark, unravels the formation of a $\pi^{0}$ and the scattering of the proton. The anti-quark couples with a quark from the proton to form a $\pi^{0}$. Figure 4.6 shows this process as a schematic diagram [67]. Later, the $\pi^{0}$ will decay into two photons, $\pi^{0} \rightarrow \gamma_{1}+\gamma_{2}$, creating a source 
of background in DVCS detections. We can identify three different cases whenever there is a $\pi^{0}$ decay:

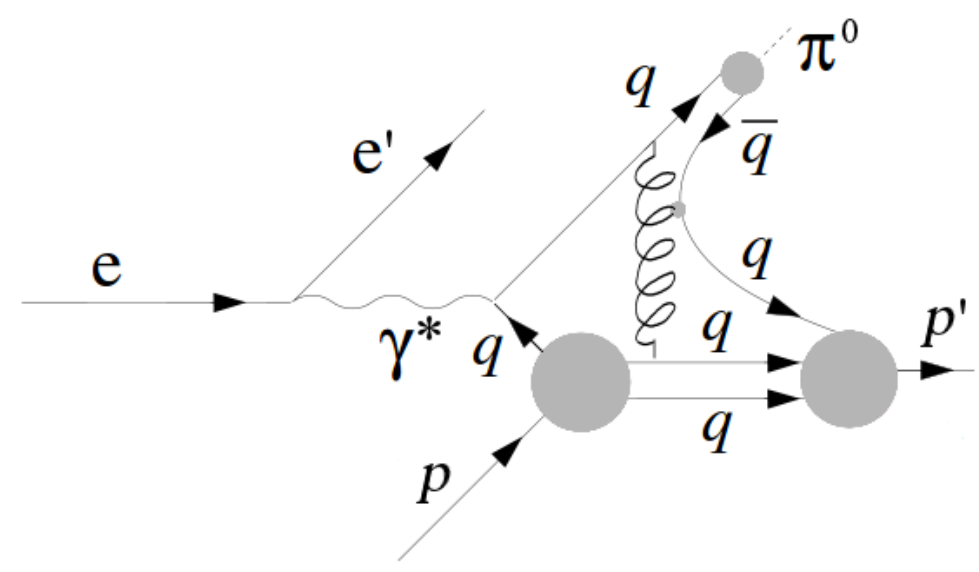

Figure 4.6: Diagram of the $\pi^{0}$ electroproduction.

- None of the photons reaches the calorimeter: then we have no detection and our data aren't affected.

- Only one, $\gamma_{1}$ or $\gamma_{2}$, is detected: this is the cause of the background. A priori, it is not possible to identify whether the photons come from a DVCS or a $\pi^{0}$ decay reaction.

- Both $\gamma_{1}$ and $\gamma_{2}$ are detected: this will not interfere with our DVCS data since the calorimeter will form two clusters. We will use these events to perform the $\pi^{0}$ subtraction.

There are different approaches to subtract the $\pi^{0}$ contribution. One could evaluate the cross section of this process within the solid angle of the experiment, and perform the subtraction. However, we opted for a simulation of randomly generated $\pi^{0}$ decays through which the previous computation is unnecessary, similar to the one used in a previous DVCS dedicated experiment in Hall A [68]. The method begins by selecting events where two clusters were formed, searching for two photons coming from $\pi^{0}$ decays. Among these events we will select those with an invariant mass for $\left(\gamma_{1}+\gamma_{2}\right)$ close to that of the $\pi^{0}$ (between $0.1 \mathrm{GeV}$ and $0.17 \mathrm{GeV}$, which is a range of $3 \sigma$ around the peak of the $M_{\pi^{0}}$ distribution studied in section 3.3.2). We use this invariant mass to create random $\pi^{0}$ decays and observe whether the resulting photons hit the calorimeter or not, in order 


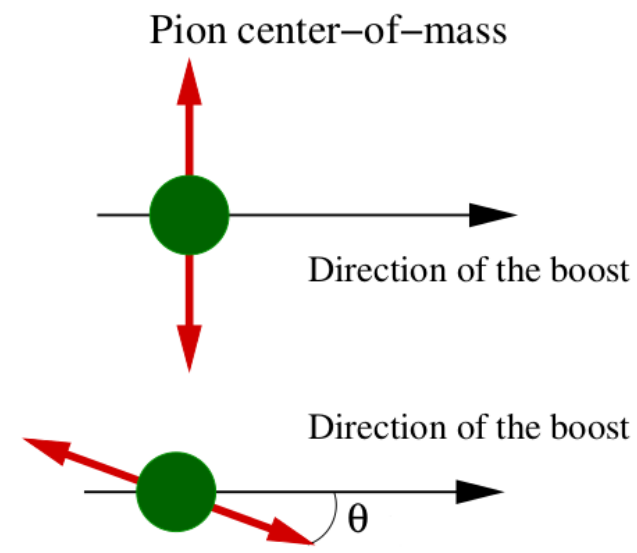

Laboratory frame
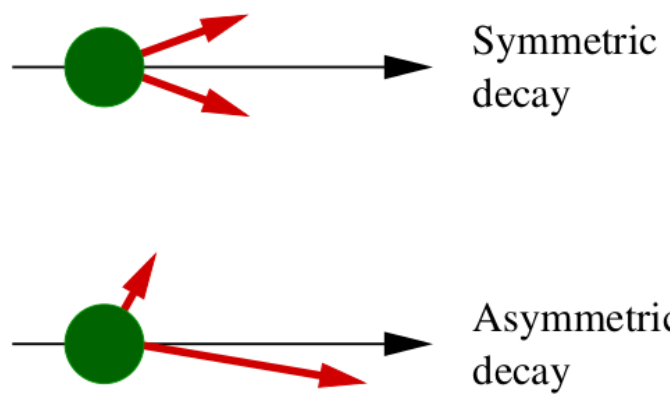

Asymmetric decay

Figure 4.7: Two different kinds of $\pi^{0}$ decays, in the pion center of mass and in the laboratory frame (after applying the corresponding boost).

to compute the contribution of this phenomenon.

The angles formed between the photons direction, in the $\pi^{0}$ center of mass frame, and the direction of the boost (the boost is applied to study the decay in the laboratory frame), are randomized 5000 times for each real $\pi^{0}$ decay we detected. This means that we now have 5000 times the amount of decays we had, all of them decaying isotropically in the center of mass frame. The next step is to apply a boost in the direction of $\gamma_{1}+\gamma_{2}$. This boost is extracted from the real event, and applied to the randomized decays, in order to check whether or not the calorimeter might have detected the resulting photons from the decay. We do this for every real decay event we selected. For the computation of the boost, we use the momentum of $\gamma_{1}+\gamma_{2}$. Figure 4.7 shows two different situations when applying the boost to a random decay. The first one, in which the direction of the boost is perpendicular to the directions of the photons in the pion center of mass frame, will lead to a symmetric decay in the laboratory frame, in which both photons will have the same energy and angle with the boost direction. In this case, the transverse momentum of the photons is $M_{\pi^{0}} / 2$ in both frames. In the lab frame, the photons total momentum will be equal to their total energy, which is just $E_{\pi^{0}} / 2$. The minimum angle of separation is given by

$$
\sin \left(\frac{\alpha_{\min }}{2}\right)=\frac{M_{\pi^{0}}}{E_{\pi^{0}}} .
$$

For the kinematics of the experiment, the maximum energy of the pions, at which the angle of separation between the photons is minimum $\left(\alpha_{\text {min }}\right)$, is about $3 \mathrm{GeV}$. This renders a minimum angle $\left(\alpha_{\min }\right)$ of $0.09 \mathrm{rad}$, which can be expressed as a separation distance of 
about $10 \mathrm{~cm}$ in the calorimeter surface (the distance from the target to the calorimeter is $1.1 \mathrm{~m}$ ). This distance ensures a separation of two blocks between each of the two photons hitting the calorimeter, and thus, the consequent formation of two different clusters if both photons are detected by the calorimeter. The minimal angle makes it impossible to detect two photons in the edges of the calorimeter, causing a source of systematic errors. It is possible to apply a fiducial cut on the calorimeter, corresponding to the area where the $\pi^{0}$ subtraction procedure is fully efficient [69]. However, the results presented in this thesis do not include this cut. In the case where the direction of the photons forms an angle $\theta \neq 90 \mathrm{deg}$ with the direction of the boost, we will find an asymmetric decay in the laboratory frame. Here it may happen that none of the photons reaches the calorimeter, or only one or both of them are detected.

The surface of the calorimeter is introduced in the simulation to observe which kind of detection we have for every randomized event. We must not forget the threshold set in the calorimeter that triggers the clustering algorithm. Even if one of the randomized photons reaches the calorimeter it may be discarded due to its low energy. The decay of the $\pi^{0}$ is placed at the target impact point, since it is not expected to cover a long distance, due to its short mean lifetime.

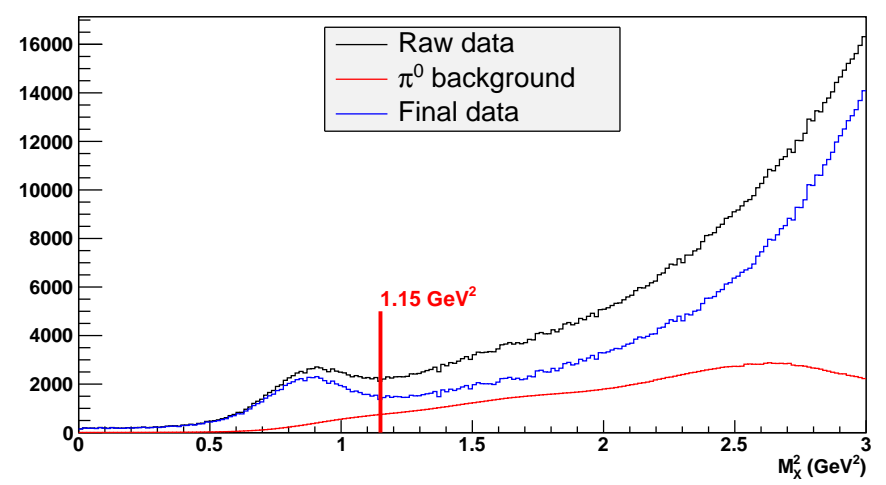

Figure 4.8: $\pi^{0}$ subtraction events as a function of $M_{X}^{2}$ (Kin 3-high).

The purpose of the randomization is to subtract each of the events in which one, and only one, of the photons from the randomized decay is detected, from the corresponding experimental bin of DVCS events, according to its kinematics. Figure 4.8 shows the $\pi^{0}$ subtraction of events as a function of $M_{X}^{2}$ (missing mass) for the one-cluster events. The $M_{X}^{2}$ variable accounts for the undetected energy in the experiment and can be expressed as

$$
M_{X}^{2}=\left(e^{\prime}+\gamma-e-p\right)^{2}
$$


All contributions from the initial and final electrons $\left(e, e^{\prime}\right)$, the real photon $(\gamma)$ and the initial proton $(p)$ are expressed in four-vectors. DVCS events are selected from one-cluster events where $M_{X}^{2}<\left(M_{p}+M_{\pi^{0}}\right)^{2}$. This means that the undetected energy belongs to a scattered proton, as we would expect from a DVCS reaction. The cut is set at $1.15 \mathrm{GeV}^{2}$. In order to subtract one histogram from another, events coming from the randomization have been applied a factor $1 / 5000$ that accounts for the number of generated decays for each event.

$$
W=\frac{1}{5000} \cdot \frac{n_{0}+n_{1}+n_{2}}{n_{2}}=\frac{1}{n_{2}}
$$

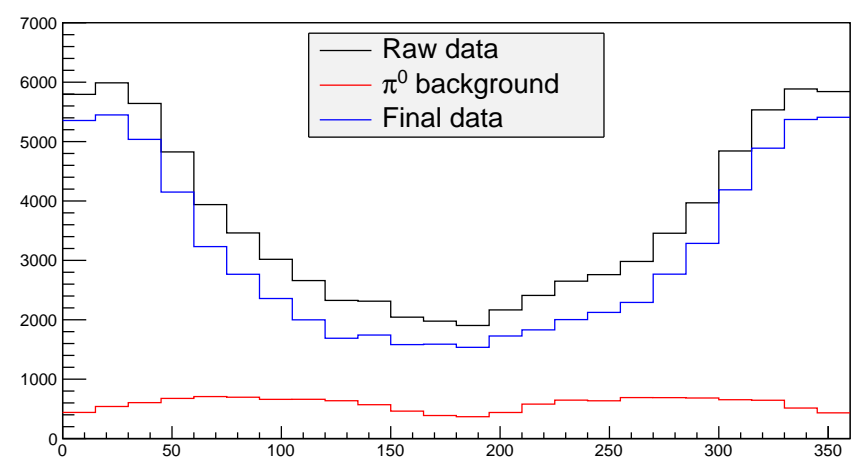

Figure 4.9: $\pi^{0}$ subtraction as a function of $\varphi_{\gamma}$, for events with $M_{X}^{2}<1.15 \mathrm{GeV}^{2}$ (Kin 3-high).

where $n_{0}, n_{1}$ and $n_{2}$ stand for the number of zero detections, one photon or two photon detections counted during the randomization $\left(n_{0}+n_{1}+n_{2}=5000\right)$. The weight $W$ takes into account that only two-cluster events, from the real events, were used for the subtraction through the factor $\left(n_{0}+n_{1}+n_{2}\right) / n_{2}$. Figure 4.9 shows the $\pi^{0}$ subtraction of events as a function of the angle between leptonic and hadronic planes in DVCS reactions $\varphi_{\gamma}$. The subtraction accounts for $16 \%$ of the raw data.

\subsubsection{Accidentals subtraction}

The accidental background refers to detections of unrelated particles in both detectors: the photon detected in the calorimeter coming from a different reaction to that of the electron being detected in the HRS. This kind of detections is impossible to distinguish from the desired ones, so we calculate their contribution using the data from the experiment. The procedure is as follows: data resulting from the waveform analysis are processed by the clustering algorithm, but instead of selecting events with a $t_{\text {event }}$ between $-3 n s$ and $3 n s$, the 
time window is shifted at two different positions, between $-11 n s$ and $-5 n s$ and between $5 n s$ and $11 n s$. Since the accidental background is time independent, it is the same no matter where the time window is set, but DVCS events don't appear in the new positions. By applying this method we find the pattern of accidental detections that we subtract from data. In a similar way as we did for $\pi^{0}$ contamination, we extract each accidental event from the corresponding experimental bin of DVCS events, according to its kinematics. We use the accidentals obtained from both time windows, adding them and weighting them by 0.5 , since both should have equal value. The purpose of having two time windows is to increase the statistics. Figures 4.10 and 4.11 show the accidental subtraction for kinematics Kin 3-high. We can observe the high rates of accidental detections around the beam direction (around $0^{\circ}$ ).

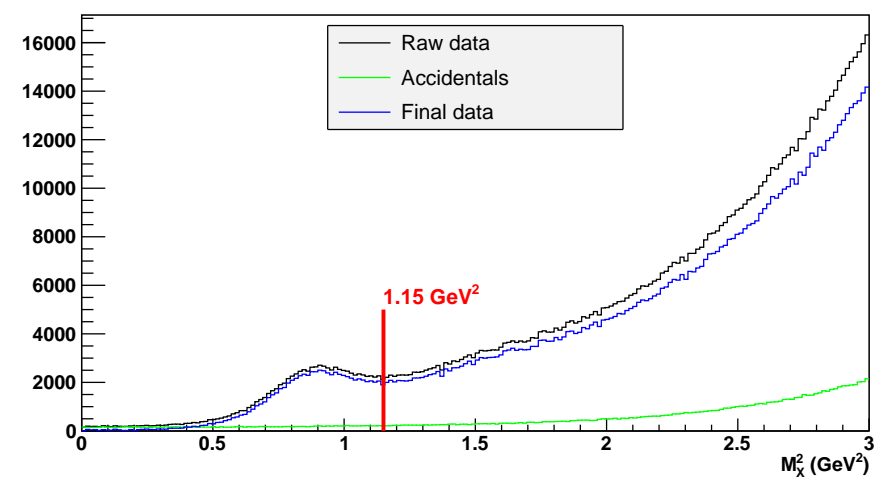

Figure 4.10: Accidental subtraction as a function of $M_{X}^{2}$ (Kin 3-high).

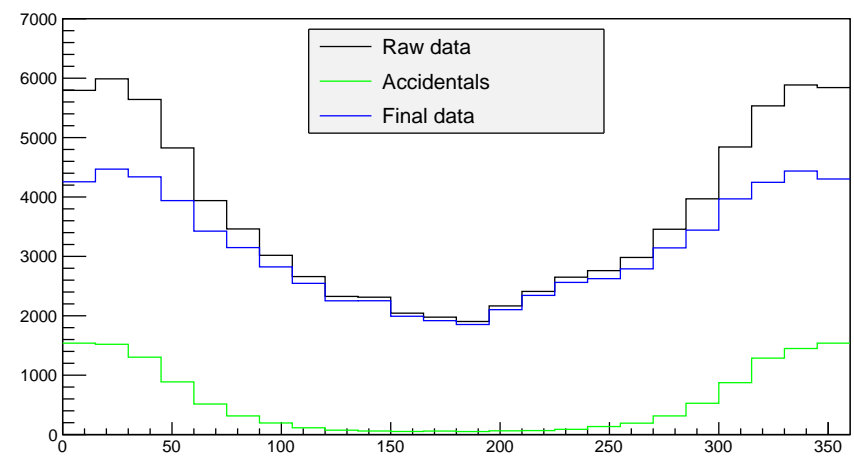

Figure 4.11: Accidental subtraction as a function of $\varphi_{\gamma}$, for events with $M_{X}^{2}<1.15 \mathrm{GeV}^{2}$ (Kin 3-high). 


\subsection{Radiative corrections}

QED radiative corrections provide an important contribution to the $e p \rightarrow e p \gamma$ reaction, as observed in the measurements in MAMI [70], the first absolute measurement of the VCS cross section on the nucleon. These corrections are of the order of $20 \%$ in the kinematics considered in [70]. The calculation of these QED radiative corrections has been studied in detail in [71] to first order in $\alpha_{e m}$, taking into account corrections from both, $\mathrm{BH}$ and VCS reactions.

Radiative corrections are usually expressed in terms of internal and external corrections. The external radiative corrections enclose the radiation of bremsstrahlung photons

by the electrons passing through a material. The external correction is applied before the initial electron impacts the proton in the $\mathrm{LH}_{2}$ target, and takes into account the bremsstrahlung created when the electron passes through the target. The energy loss of the electron due to this phenomenon is calculated through the approximation [72]

$$
I_{e x t}\left(E_{0}, \Delta E, d\right)=\frac{b d}{\Gamma(1+b d)}\left(\frac{\Delta E}{E_{0}}\right)^{b d}\left[\frac{1}{\Delta E}\left(1-\frac{\Delta E}{E_{0}}+\frac{3}{4}\left(\frac{\Delta E}{E_{0}}\right)^{2}\right)\right],
$$

where $E_{0}$ is the initial kinetic energy of the electron ( $E_{b}$ in our case), $b \simeq 4 / 3$, and $d$ is the thickness of the material expressed in radiation length units $(d<0.05$ in the approximation). The acceptance is usually computed through a Monte Carlo simulation of the experimental apparatus, where all the resolutions of all detectors are included. In the case of the simulation the energy loss $\Delta E$ is generated randomly through the expression [73]

$$
E^{e x t}=E_{0} R_{0}^{1 / b d},
$$

Here, $R_{0}$ is a uniform random variable from the interval $[0,1]$. This generates an external radiation distribution

$$
I_{e x t}\left(E_{0}, \Delta E, d\right)=\frac{b d}{\Delta E}\left(\frac{\Delta E}{E_{0}}\right)^{b d}
$$

which is an approximation of (4.10) that reproduces the dominant $1 / \Delta E$ behaviour. On the other hand, the internal radiative corrections can be divided into two different contributions, virtual corrections and real corrections. The real radiative diagrams include the emission of a real photon by any electron line while the virtual corrections include vertex and vacuum polarization corrections. The experimental cross section can be expressed as

$$
\left.\frac{d \sigma}{d \Omega}\right|_{\text {Exp }}=\left.\frac{d \sigma}{d \Omega}\right|_{\text {Born }}\left[1+\delta_{\text {Virtual }}+\delta_{\text {Real }}(\Delta E)\right],
$$


where the "Born cross section" is the hypothetical cross section that would be valid if QED stopped at the lowest order of perturbation theory. The individual terms for each of the contributions of the internal radiative diagrams read [71]

$$
\begin{aligned}
\delta_{\text {Vacuum }}= & \frac{2 \alpha}{3 \pi}\left[\ln \left(\frac{\mathcal{Q}^{2}}{m_{e}^{2}}\right)-\frac{5}{3}\right] \\
\delta_{\text {Vertex }}= & \frac{\alpha}{\pi}\left[\frac{3}{2} \ln \left(\frac{\mathcal{Q}^{2}}{m_{e}^{2}}\right)-2-\frac{1}{2} \ln ^{2}\left(\frac{\mathcal{Q}^{2}}{m_{e}^{2}}\right)+\frac{\pi^{2}}{6}\right] \\
\delta_{\text {Real }}= & \frac{\alpha}{\pi}\left\{2 \ln \left(\frac{\Delta E}{\sqrt{E E^{\prime}}}\right)\left[\ln \left(\frac{\mathcal{Q}^{2}}{m_{e}^{2}}\right)-1\right]\right. \\
& \left.-\frac{1}{2} \ln ^{2}\left(\frac{E}{E^{\prime}}\right)+\frac{1}{2} \ln \left(\frac{\mathcal{Q}^{2}}{m_{e}^{2}}\right)-\frac{\pi^{2}}{3}+S p\left(\cos ^{2} \frac{\theta_{e}}{2}\right)\right\}
\end{aligned}
$$

Note that the term $\delta_{\text {Real }}$ has a contribution that depends on the integration cutoff $\Delta E$, as well as a term independent of $\Delta E$. These terms originate from photons of two different kinematic domains: soft photons, whose energy is less than the experimental resolution (originate the $\Delta E$ independent term), and photons whose energy is resolvable, and might generate events that are included in the integrated cross section. These photons generate a radiative tail in the missing mass and must be taken into account in the computation of the solid angle or acceptance. In the case of the Monte Carlo simulation these corrections are computed using the equivalent radiator technique [75], which assumes that the internal radiation is equivalent to placing one radiator before the scattering and another radiator of the same thickness after the scattering. The correction is applied twice, once before the scattering $\left(E_{1}^{\text {int }}\right)$ and once after the scattering $\left(E_{2}^{\text {int }}\right)$.

$$
\begin{aligned}
& E_{1}^{i n t}=E^{e x t} R_{1}^{2 / \nu} \\
& E_{2}^{i n t}=E^{\prime} R_{2}^{2 / \nu}
\end{aligned} \quad \text { with } \quad \nu=\frac{\alpha}{\pi}\left[\ln \left(\frac{\mathcal{Q}^{2}}{m_{e}^{2}}\right)-1\right] .
$$

$\nu$ plays the role of the equivalent radiator thickness $(b d) . E^{\prime}$ is the energy of the electron after the scattering and $R_{1}$ and $R_{2}$ are uniform random variables from the interval $[0,1]$.

The virtual radiative corrections do not modify the kinematics of the reaction. The diagrams of the processes involved in the virtual corrections are shown in figure 4.12. These are one-loop virtual radiative corrections originating from the electron side and, for the case of $\mathrm{BH}$, they can be calculated model-independently. The corrections to the $\mathrm{BH}$ process contain vertex corrections: figs. 4.12 (V1i - V3i) and (V1f - V3f); electron self-energy corrections: figs. 4.12 (Si, Sf); and vacuum polarization corrections: figs. 4.12 (P1i, P1f). The captions of the figures indicate whether the photon in the ep $\rightarrow$ ep $\gamma$ 
reaction is emitted from the initial (i) electron or from the final (f) electron. For the case of DVCS processes, the virtual radiative corrections can be divided into model dependent and model independent. Those which can be calculated model-independently, consist on the vertex diagram shown in figure 4.12 (V4), and the vacuum polarization diagram shown in $4.12(\mathrm{P} 2)$. The virtual corrections along with the contribution from soft photons of the real corrections are nearly constant for the phase space of interest. They can be applied as a constant factor to the measured data, which in our case has a value of 1/1.1 [74].
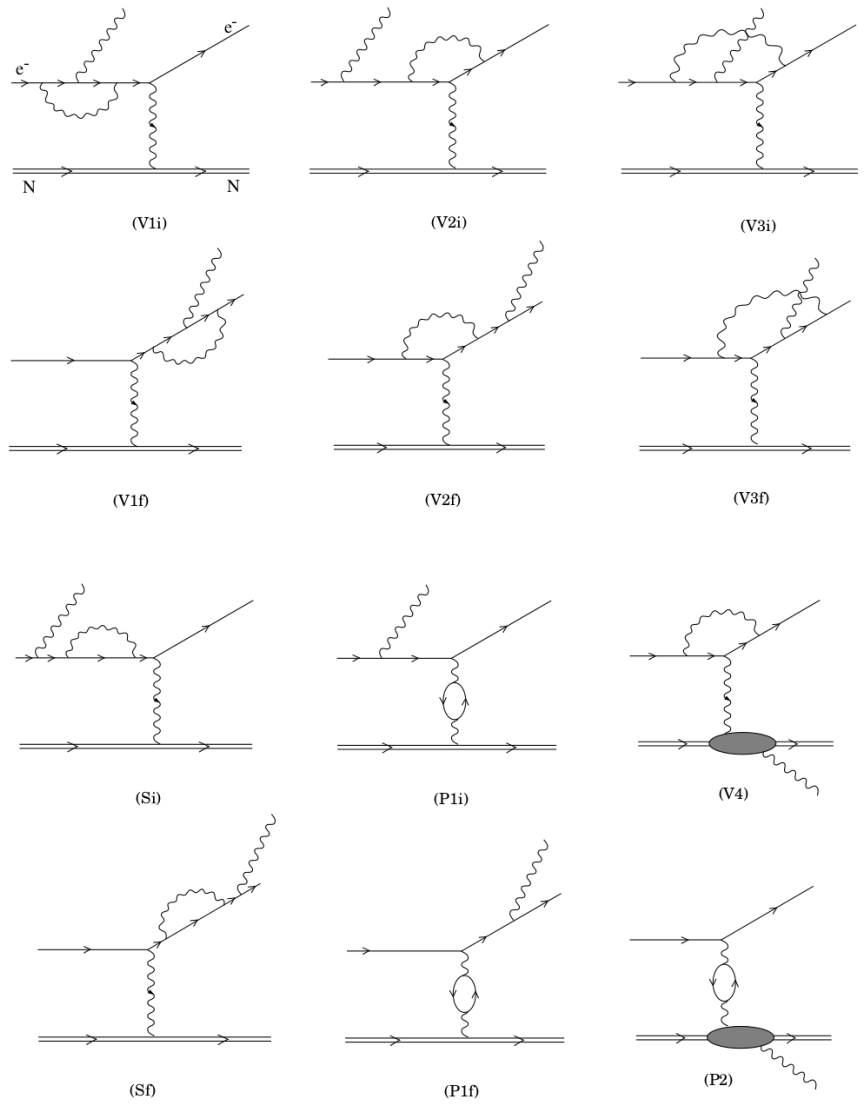

Figure 4.12: First order virtual photon radiative corrections to the $e p \rightarrow e p \gamma$ reaction.

\subsection{Multicluster correction}

Until now, all events coming from DVCS reactions have been selected from one-cluster events. We have to take into account that there might be DVCS events processed as twocluster events, due to the formation of accidental clusters besides the clusters formed by a DVCS photon. Those events should not be discarded due to our analysis scheme, therefore 
we treat every signal from the two-cluster events package as DVCS event candidates. By this, we mean that all the previous cuts are applied to the selection, including the cut in the missing mass. Since we have two detections for every two-cluster event, $M_{X}^{2}$ is calculated as if we only had detected one of them. We do this for both photons of the same event. Finally, we add the contribution from two-cluster events to our initial onecluster DVCS events. The contribution of this correction accounts for about $7 \%$ from the total raw data. Figures 4.13 and 4.14 show the multicluster correction applied for kinematics Kin 3-high.

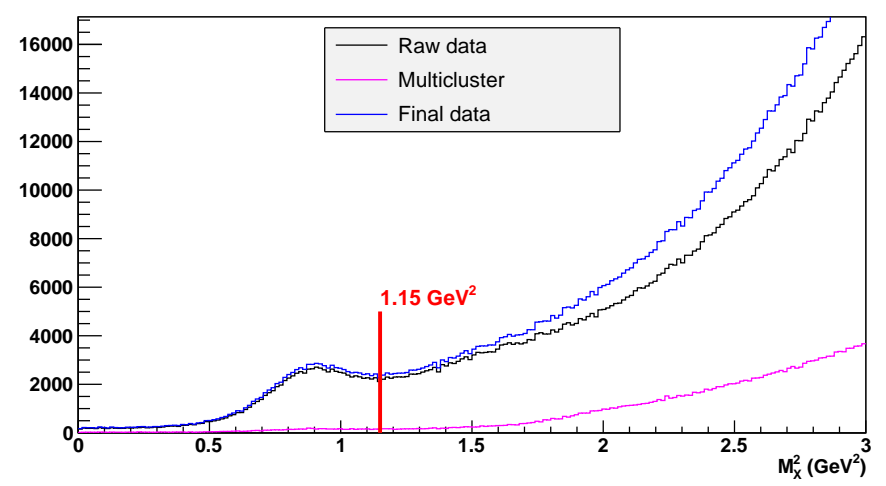

Figure 4.13: Multicluster correction as a function of $M_{X}^{2}$ (Kin 3-high).

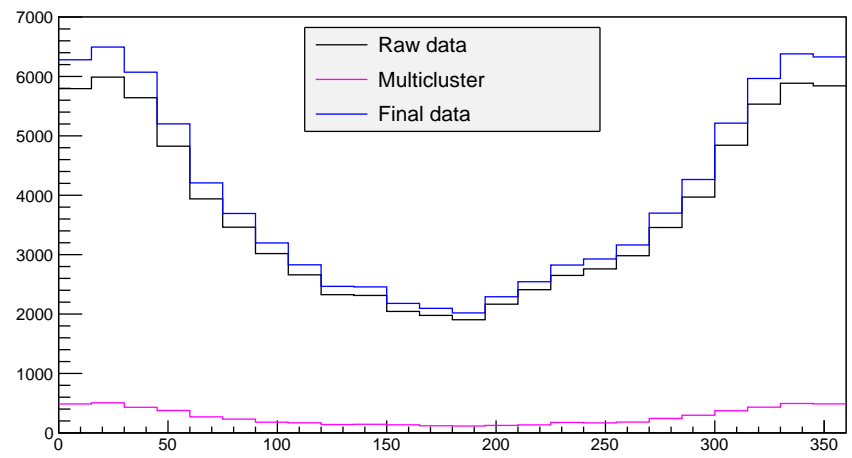

Figure 4.14: Multicluster correction as a function of $\varphi_{\gamma}$, for events with $M_{X}^{2}<1.15 \mathrm{GeV}^{2}$ (Kin 3-high). 


\subsection{Final data}

The final data (for Kin 3-high) used for the extraction of observables that will lead to a DVCS cross section calculation are shown in Figures 4.15 and 4.16. These figures include the cuts, background subtraction, and corrections mentioned before. In all the figures where $M_{X}^{2}$ is plotted we can observe a peak around $M_{p}^{2}$. This is what one would expect since the recoil proton is not detected. All experimental runs are analyzed individually (including the background subtraction) and the resulting data are put together according to their kinematics.

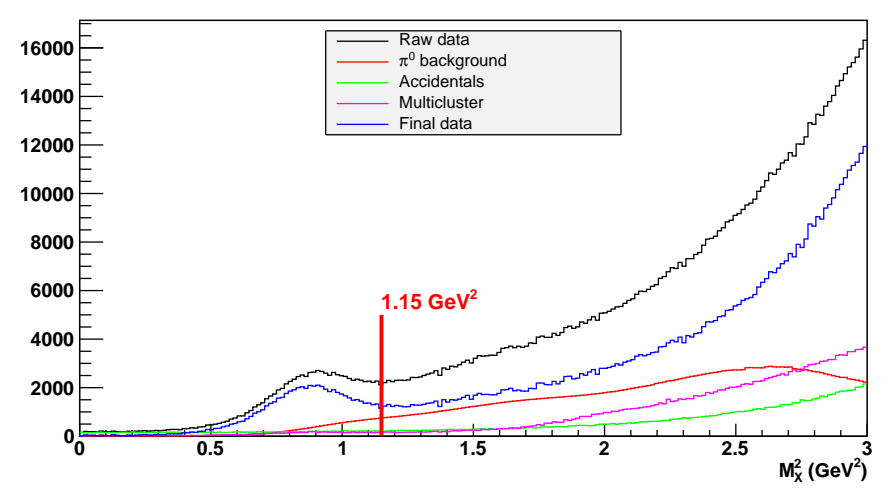

Figure 4.15: Final data as a function of $M_{X}^{2}$ (Kin 3-high).

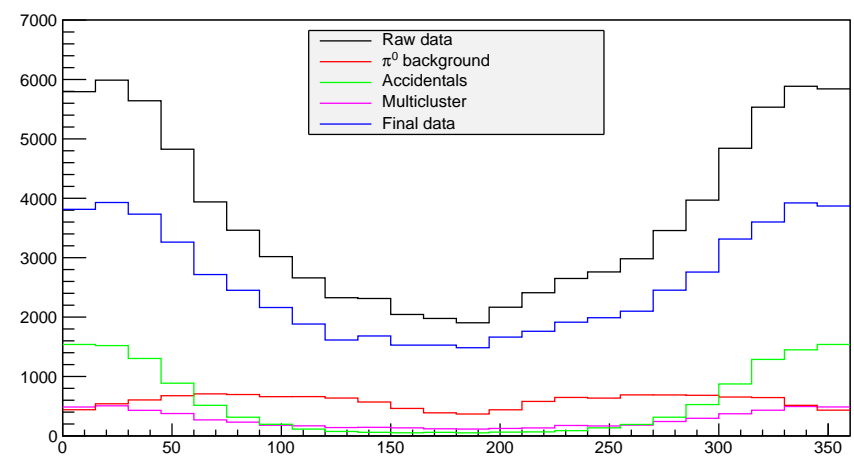

Figure 4.16: Final data as a function of $\varphi_{\gamma}$, for events with $M_{X}^{2}<1.15 \mathrm{GeV}^{2}$ (Kin 3-high). 


\subsection{Systematic errors}

Systematic errors are errors which are not determined by chance but are introduced by inaccuracies in the equipment or flaws in the design of the experiment. This type of errors cannot be estimated by repeating the experiment with the same equipment. One source of error comes from the cut applied in the "Missing mass" variable. This cut takes the form of $\left(M_{p}+M_{\pi^{0}}\right)^{2}$, in order to constrain the selection of events to those in which the missing mass corresponds to a scattered proton only. Ideally, the cross section is independent of this cut. Therefore, if we varied the value of the cut $\left(M_{X}^{2}<1.1 \mathrm{GeV}^{2}\right.$ for this matter) the resulting cross section should not differ from the original one. The variation between both cross sections is an estimation of the systematic error induced by this cut. Figure 4.17 shows the ratio between lepton photoproduction cross sections obtained with two different cuts for $M_{X}^{2}$ (for five different bins in $t$ ). The variation between both is, in overall, about $2 \%$. There are other sources of systematic errors in the experiment, such as the one due to the edges of the calorimeter, or the uncertainty in the calorimeter energy threshold. However, I did not have the time to address these issues during the duration of my thesis, but it remains as work to do.

A study of the DIS cross section has been performed in [69]. The experimental cross sections have been compared to a parametrization of the DIS cross section with the kinematics employed in the experiment. The discrepancies in the values of the cross sections vary up to a value of $3.5 \%$, depending on the studied kinematic. We can use this information to evaluate, in a preliminary way, the impact of the systematic errors in the DVCS experiment. The study of DIS reactions does not include the electromagnetic calorimeter, but apart from that, the geometry and efficiencies of the rest of the devices, included in the computation of the cross section, are the same as for DVCS reactions. Then, the major difference between the systematic errors appearing in DIS and DVCS reactions would come from the efficiency and acceptance of the electromagnetic calorimeter. This device collects $99.9 \%$ of the electromagnetic shower created by the detected photons, and, since the poor energy reconstruction in the borders has been addressed, we can conclude that the contribution of systematic errors from these factors (calorimeter efficiency and acceptance) is negligible. Table 4.1 shows the systematic errors studied in [69]. 

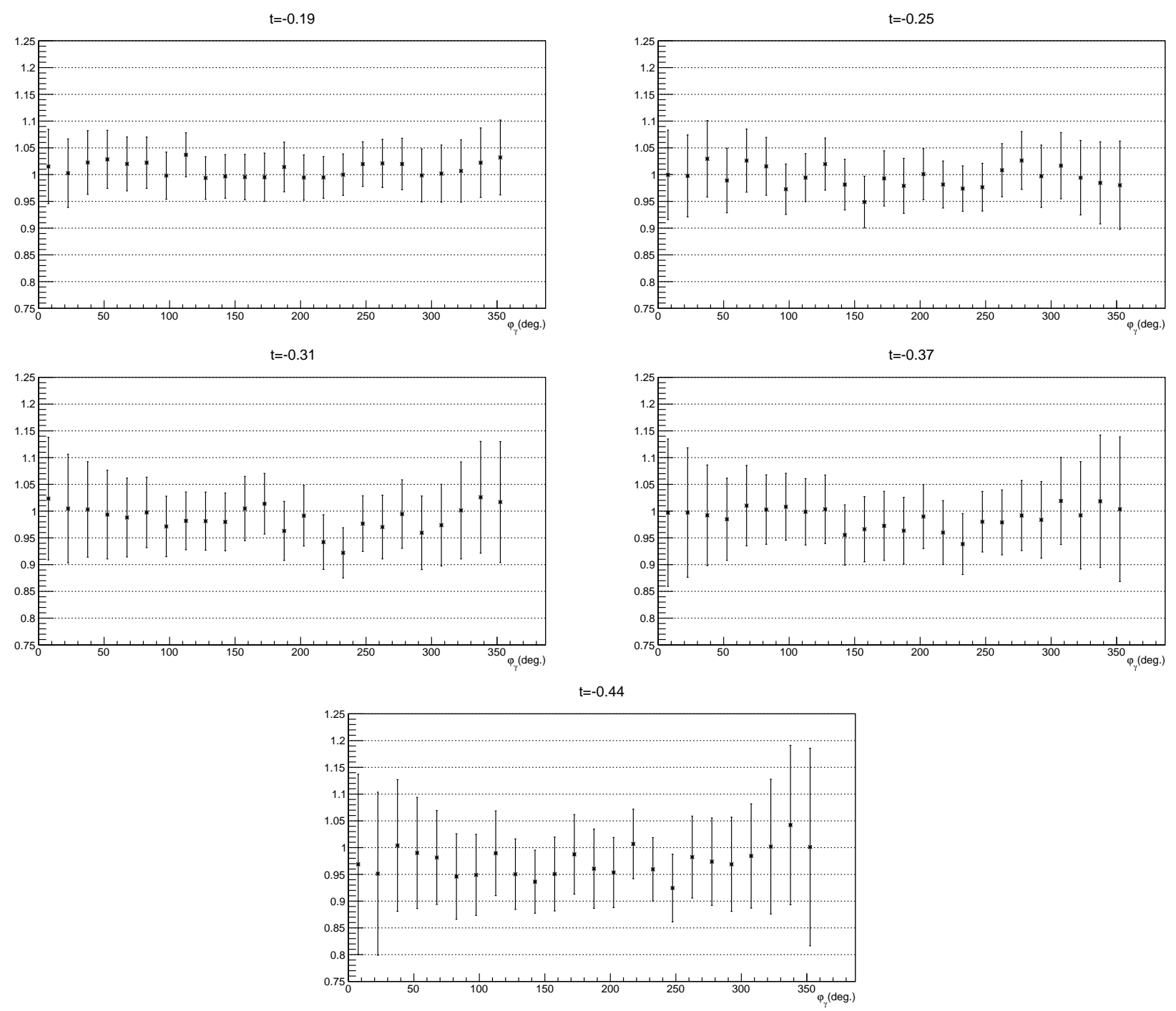

Figure 4.17: Comparison of the experimental cross section (Kin 3-high) at two different cuts for $M_{X}^{2}$, for five different bins in $t$. The plots show the ratio of the experimental points with $M_{X}^{2}<1.1 \mathrm{GeV}^{2}$ and the experimental points with $M_{X}^{2}<1.15 \mathrm{GeV}^{2}$. Error bars for each cross section have been obtained by fitting experimental data to a Monte Carlo simulation (this is addressed later in the chapter).

\begin{tabular}{|c|c|}
\hline Source & Systematic error \\
\hline Charge & $2 \%$ \\
Dead time & $1 \%$ \\
HRS efficiency & $1 \%$ \\
HRS acceptance & $1 \%$ \\
Multitrack events & $0.5 \%$ \\
Radiative corrections & $2 \%$ \\
\hline Quadratic total & $3.5 \%$ \\
\hline
\end{tabular}

Table 4.1: Systematic errors coming from the study of DIS data, taken concurrently with the experiment E07-007. 
The main contributions to the systematic errors come from the fluctuation in the measurement of the beam charge and the radiative corrections. The fluctuation in the charge measurement appears as a result of a recalibration of the BCM during the experiment. The QED radiative corrections studied in [69] are for the DIS case. Besides all these corrections we must include the contribution coming from the cut in the missing mass. We studied this systematic error for the Kin 3-high kinematic, and found a value of $2 \%$ for this case. Further study should be performed for this matter, analysing the rest of the kinematics and including different values for the cuts. With all we could expect a total systematic error of about $5 \%$.

\subsection{Monte Carlo Simulation}

A Monte Carlo simulation of the experiment was conducted in order to include geometry and resolution effects [64], such as the HRS acceptance, in the computation of the cross sections. It was performed using GEANT4, which is a platform for the simulation of the passage of particles through matter [76].

The simulation includes the main devices employed during the experiment: the scattering chamber along with the upstream and downstream beam pipes, the target, the calorimeter, the shieldings that protected the calorimeter from secondary particles, and the HRS. The latter is implemented as a virtual surface, simulating the HRS front window, which extracted the energy of electrons that reached it. For all the other components, the simulation requires their geometry, the material they are made of (along with its properties: density...), and their relative position to one another. Also, the properties of the area enclosed by them are included (vacuum, air...). In this simulation the calorimeter is very detailed. It is composed of the 208 blocks, forming 13 columns and 16 rows, including their geometry and material properties, as well as wrapping materials, PM carriers, screws... and the frame that holds them together.

In the experiment only the scattered electron and the photon were detected. These particles are the ones tracked by the simulation. Their generation is done using an event generator which generates events in DVCS kinematics. In order to match the specific kinematics used in the experiment, the beam energy as well as the HRS angle and central momentum are included in the simulation. Also, the calorimeter angle and the distance from the target have to match that of the experiment. The scattered electrons are obtained by generating $\mathcal{Q}^{2}$ in the range $\left[\mathcal{Q}_{\text {min }}^{2}: \mathcal{Q}_{\text {max }}^{2}\right]$, where $\mathcal{Q}_{\text {min }}^{2}$ and $\mathcal{Q}_{\text {max }}^{2}$ are constrained by the HRS angle and momentum. The kinematic variable $t$ is also uniformly generated between a range $\left[t_{\min }: t_{\max }\right]$ of kinematically allowed values. The last step of the simulation is to generate a uniform random angle around the beam axis within the vertical 


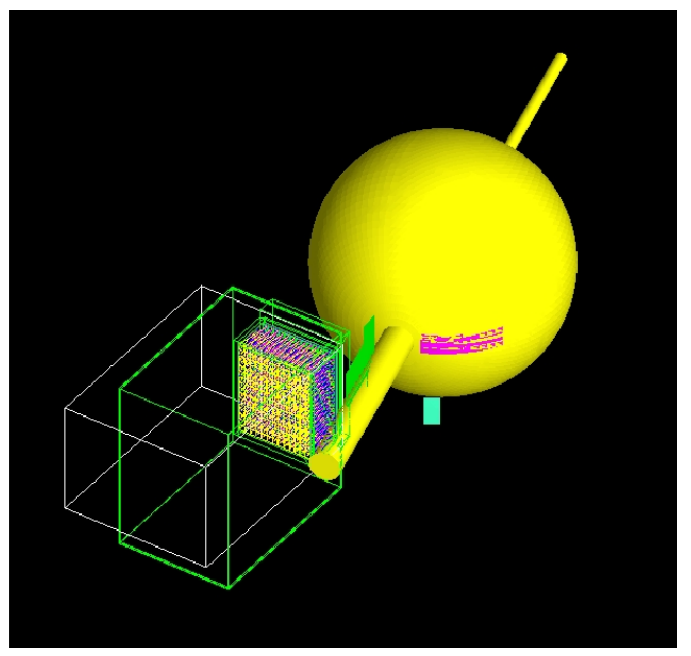

Figure 4.18: Simulation setup. Including vacuum chamber and beam pipes (yellow), Kapton window (magenta), calorimeter shielding (green), HRS front window (light-blue) and calorimeter.

angular range of the HRS, and check whether the electron and photon are detected.
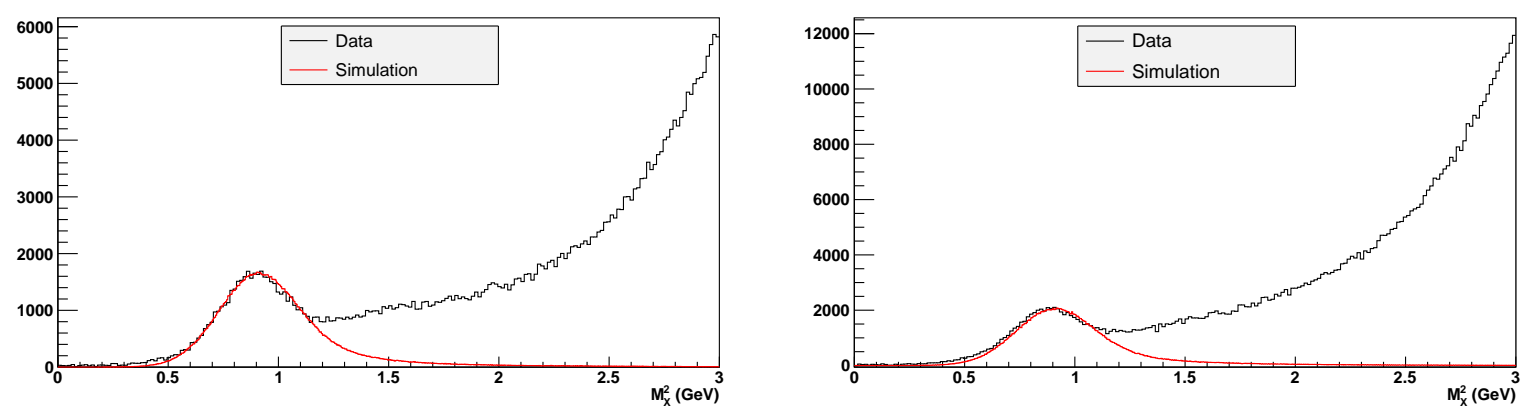

Figure 4.19: Comparison between data and simulation through the missing mass variable. Left: Kin 3-high, right: Kin 3-low. The simulation line is scaled in order to match the data.

In order to be able to compare experiment results to the Monte Carlo simulation, all the cuts mentioned in previous sections are applied to the simulation as well, including the cut in the $M_{X}^{2}$ and the radiative corrections. Finally, we obtain the number of DVCS events registered by the simulation as a function of the angle $\varphi_{\gamma}$ which we will use for the extraction of observables from data. We observe in figure 4.19 how the simulation 
matches the data in the region of interest. Note the peak around $M_{p}^{2} \simeq 0.88(\mathrm{GeV})$ for the recoiled proton.

\subsection{Extraction of observables}

Following the theoretical development from chapter one, we express the photon electroproduction helicity-independent cross section, for the case of an unpolarized target, as

$$
\frac{d^{4} \sigma}{d x d\left|\Delta^{2}\right| d \phi d \mathcal{Q}^{2}}=\Gamma^{G}\left|\mathcal{T}_{B H}\right|^{2}+\Gamma^{1} \mathcal{C}_{\text {unp }}^{\mathcal{I}}(\mathcal{F})+\Gamma^{2} \Delta \mathcal{C}_{\text {unp }}^{\mathcal{I}}(\mathcal{F})+\Gamma^{3} \mathcal{C}_{\text {unp }}^{\mathcal{I}}\left(\mathcal{F}^{\text {eff }}\right)
$$

In this case, our set of unknowns will include the observables $\mathcal{C}_{\text {unp }}^{\mathcal{I}}(\mathcal{F}), \Delta \mathcal{C}_{\text {unp }}^{\mathcal{I}}(\mathcal{F})$ and $\mathcal{C}_{\text {unp }}^{\mathcal{I}}\left(\mathcal{F}^{e f f}\right)$. Note that in 4.18 the $\mathcal{C}_{\text {unp }}^{D V C S}\left(\mathcal{F}, \mathcal{F}^{*}\right)$ term is omitted from the expansion (see (1.61)). Its contribution will be embedded in the resulting calculation of the observables $\mathcal{C}_{\text {unp }}^{\mathcal{I}}(\mathcal{F})$ and $\Delta \mathcal{C}_{\text {unp }}^{\mathcal{I}}(\mathcal{F})$, and the disentanglement of these three terms will be the goal of the subsequent "Rosenbluth separation". Additionally, our cross section depends only on four variables. We integrated over $\varphi$, since this variable contains no physics, and replaced the cross section dependence on the kinematic variable $y$ by the dependence on $\mathcal{Q}^{2}$, both related through the expression $\mathcal{Q}=E_{b} \varepsilon y$. The different kinematic factors multiplying the CFFs read:

$$
\begin{aligned}
& \Gamma^{1}=\Gamma^{G} \cdot \Gamma^{\mathcal{I}} \cdot\left\{-8(2-y)\left[\frac{(2-y)^{2}}{1-y} K^{2}+\frac{\Delta^{2}}{\mathcal{Q}^{2}}(1-y)\left(2-x_{B}\right)\right]-8 K\left(2-2 y-y^{2}\right) \cos (\phi)\right\} \\
& \Gamma^{2}=\Gamma^{G} \cdot \Gamma^{\mathcal{I}} \cdot\left\{-8(2-y) \frac{\Delta^{2}}{\mathcal{Q}^{2}}(1-y)\left(2-x_{B}\right)\right\} \\
& \Gamma^{3}=\Gamma^{G} \cdot \Gamma^{\mathcal{I}} \cdot\left\{\frac{-16 K^{2}}{2-x_{B}}(2-y) \cos (2 \phi)\right\}
\end{aligned}
$$

where $\Gamma^{G}$ is a global factor of the photon leptoproduction cross section and $\Gamma^{\mathcal{I}}$ is the factor multiplying the Fourier decomposition of the interference amplitude,

$$
\Gamma^{G}=\frac{\alpha^{3} x_{B} y}{16 \pi^{2} \mathcal{Q}^{2} \sqrt{1+\varepsilon^{2}}} \cdot \frac{\pi}{E_{b} x_{B} M_{p}} \quad \quad \Gamma^{\mathcal{I}}=\frac{1}{x_{B} y^{3} \Delta^{2} \mathcal{P}_{1}(\phi) \mathcal{P}_{2}(\phi)}
$$


The collected data from the experiment are in terms of event counts, which for a determined phase space bin $N_{i}$, we can relate to the average cross section over the bin,

$$
N_{i}=\mathcal{L} \int_{i} \frac{d \sigma}{d \Omega} d \Omega=\mathcal{L} \frac{\int_{i} \frac{d \sigma}{d \Omega} d \Omega}{\int_{i} d \Omega} \int_{i} d \Omega=\mathcal{L}\left\langle\frac{d \sigma}{d \Omega}\right\rangle_{i} \Delta \Omega_{i}
$$

were $\mathcal{L}$ stands for the luminosity of the electron beam. For electrons of a total charge $Q$ passing through a $\mathrm{LH}_{2}$ target with a density $\rho$ and length $l$, the integrated luminosity is given by

$$
\int \mathcal{L} d t=\frac{Q}{e} \frac{N_{A} \cdot \rho \cdot l}{A_{H}}
$$

where $e=1.602 \cdot 10^{-19} \mathrm{C}$ is the electron charge, $A_{H}=1.0079 \mathrm{~g} / \mathrm{mol}$ is the atomic mass of the hydrogen, and $N_{A}=6.022 \cdot 10^{23} \mathrm{~mol}^{-1}$ is Avogadro's number. The charge $Q$ passing through the target in a run is given by the BCM (section 2.3.2). The length of the $\mathrm{LH}_{2}$ target is $15 \mathrm{~cm}$, with a density of $0.0723 \mathrm{~g} / \mathrm{cm}^{3}$ for the experiment conditions (section 2.4).

At this point we can correlate our data to the photon leptoproduction cross section, thanks to (4.23). In order to include the detection geometry and the resolution effects in our calculus we employ a Monte Carlo (MC) simulation. We will use the simulation altogether with the experimental data, to fit the parameters of (4.18), and compute the cross section. When applying a binning to the kinematic variables, some issues arise. The binning reduces the impact of the variation of the kinematic factors, but we must take into account the effects of bin migration, caused by the resolution of the detectors. The study of this bin migration is performed by the Monte Carlo simulation. By defining the kinematic variables on the vertex, $x_{v}$, and observing the distribution of the variables reconstructed by the detectors, $x_{e}$, for every simulated event, we can implement the mapping function,

$$
M\left(x_{v} \mid x_{e}\right),
$$

which represents the conditional probability to observe an event at the kinematic point $x_{e}$, starting from the vertex point $x_{v}$. For binning purposes we define the binning vectors $j_{v}$ and $j_{e}$, which label a set of kinematic variables from $x_{v}$ and $x_{e}$ respectively, and introduce the binning in the variables $t$ and $\varphi_{\gamma}$. We will represent the photon leptoproduction cross section in terms of these two variables. We can now rewrite (4.23) using (4.18) and the binning vectors,

$$
N\left(j_{v}\right)=\mathcal{L} \int_{x_{v} \in \operatorname{Bin}\left(j_{v}\right)} \sum_{\Lambda=0}^{3} \Gamma^{\Lambda}\left(x_{v}\right) X_{j_{v}}^{\Lambda} d x_{v}=\mathcal{L} \sum_{\Lambda=0}^{3} X_{j_{v}}^{\Lambda} \int_{x_{v} \in \operatorname{Bin}\left(j_{v}\right)} \Gamma^{\Lambda}\left(x_{v}\right) d x_{v}
$$


where we used that

$$
d^{4} \sigma\left(x_{v}\right)=\sum_{\Lambda=0}^{3} \Gamma^{\Lambda}\left(x_{v}\right) X_{j_{v}}^{\Lambda} \quad \text { with } \quad\left\{\begin{array}{l}
\Gamma^{0}\left(x_{v}\right) X_{j_{v}}^{0}=\Gamma^{G}\left(x_{v}\right)\left|\mathcal{T}_{B H}\right|_{j_{v}}^{2} \\
\Gamma^{1}\left(x_{v}\right) X_{j_{v}}^{1}=\Gamma^{1}\left(x_{v}\right) \mathcal{C}_{\text {unp }}^{\mathcal{I}}(\mathcal{F})_{j_{v}} \\
\Gamma^{2}\left(x_{v}\right) X_{j_{v}}^{2}=\Gamma^{2}\left(x_{v}\right) \Delta \mathcal{C}_{u n p}^{\mathcal{I}}(\mathcal{F})_{j_{v}} \\
\Gamma^{3}\left(x_{v}\right) X_{j_{v}}^{3}=\Gamma^{3}\left(x_{v}\right) \mathcal{C}_{\text {unp }}^{\mathcal{I}}\left(\mathcal{F}^{e f f}\right)_{j_{v}}
\end{array}\right\}
$$

For the case of the bins containing the kinematic variables reconstructed by the detectors we have

$$
\begin{aligned}
N\left(j_{e}\right) & =\int_{x_{e} \in \operatorname{Bin}\left(j_{e}\right)} \sum_{j_{v}} N\left(j_{v}\right) M\left(x_{e} \mid x_{v}\right) d x_{e} \\
& =\mathcal{L} \sum_{j_{v}} \sum_{\Lambda=0}^{3} X_{j_{v}}^{\Lambda} \int_{x_{e} \in \operatorname{Bin}\left(j_{e}\right)} \int_{x_{v} \in \operatorname{Bin}\left(j_{v}\right)} \Gamma^{\Lambda}\left(x_{v}\right) M\left(x_{e} \mid x_{v}\right) d x_{e} d x_{v} .
\end{aligned}
$$

Here we redefine our mapping function so it represents the conditional probability of bin migration between $j_{e}$ and $j_{v}$,

$$
M_{j_{e}, j_{v}}^{\Lambda}=\int_{x_{e} \in \operatorname{Bin}\left(j_{e}\right)} \int_{x_{v} \in \operatorname{Bin}\left(j_{v}\right)} \Gamma^{\Lambda}\left(x_{v}\right) M\left(x_{e} \mid x_{v}\right) d x_{e} d x_{v},
$$

which leads us to the more compact relation for the number of counts in the experimental bin, $N\left(j_{e}\right)$, which being the case of a Monte Carlo simulation we will rename by $N^{M C}\left(j_{e}\right)$, in order to differentiate it from the real detections in the laboratory, $N^{\operatorname{Exp}}\left(j_{e}\right)$.

$$
N^{M C}\left(j_{e}\right)=\mathcal{L} \sum_{j_{v}, \Lambda} M_{j_{e}, j_{v}}^{\Lambda} X_{j_{v}}^{\Lambda} .
$$

We are now in position of extracting the observables $X_{j_{v}}^{\Lambda}$ by fitting our experimental data $N^{E x p}\left(j_{e}\right)$, using the Monte Carlo simulation $N^{M C}\left(j_{e}\right)$. We begin by defining a $\chi^{2}$ that relates both measures,

$$
\chi^{2}=\sum_{j_{e}} \frac{\left[N^{E x p}\left(j_{e}\right)-N^{M C}\left(j_{e}\right)\right]^{2}}{\left[\sigma^{E x p}\left(j_{e}\right)\right]^{2}} .
$$

Here we introduced the error for the number of experimental counts for a bin $j_{e}, \sigma^{E x p}\left(j_{e}\right)$, which is simply $\sqrt{N^{E x p}\left(j_{e}\right)}$. To obtain the CFFs we minimize our $\chi^{2}$ with respect to the $X_{j_{v}}^{\Lambda}$ coefficients,

$$
0=-\left.\frac{1}{2} \frac{\partial \chi^{2}}{\partial X_{j_{v}}^{\Lambda}}\right|_{\bar{X}_{j_{v}}^{\Lambda}}
$$


where the coefficients $\bar{X}_{j_{v}}^{\Lambda}$ are defined as the values of $X_{j_{v}}^{\Lambda}$ that minimize our $\chi^{2}$. Substituting the definition of $N^{M C}\left(j_{e}\right)$ from (4.30) into our minimization renders

$$
0=\sum_{j_{e}} \mathcal{L} M_{j_{e}, j_{v}}^{\Lambda} \frac{\mathcal{L} \sum_{j_{v}^{\prime}, \Lambda^{\prime}} M_{j_{e}^{\prime}, j_{v}^{\prime}}^{\Lambda^{\prime}} \bar{X}_{j_{v}^{\prime}}^{\Lambda^{\prime}}-N^{E x p}\left(j_{e}\right)}{\left[\sigma^{E x p}\left(j_{e}\right)\right]^{2}} .
$$

We can express the last equation in a matricial form by defining the matrix $\alpha_{j_{v}, j_{v}^{\prime}}^{\Lambda, \Lambda^{\prime}}$ and the vector $\beta_{j_{v}}^{\Lambda}$ through the following expressions:

$$
\begin{aligned}
& \alpha_{j_{v}, j_{v}^{\prime}}^{\Lambda, \Lambda^{\prime}}=\sum_{j_{e}} \mathcal{L}^{2} \frac{M_{j_{e}, j_{v}}^{\Lambda} M_{j_{e}^{\prime}, j_{v}^{\prime}}^{\Lambda^{\prime}}}{\left[\sigma^{\operatorname{Exp}}\left(j_{e}\right)\right]^{2}}, \\
& \beta_{j_{v}}^{\Lambda}=\sum_{j_{e}} \mathcal{L} \frac{M_{j_{e}, j_{v}}^{\Lambda} N^{\operatorname{Exp}}\left(j_{e}\right)}{\left[\sigma^{\operatorname{Exp}}\left(j_{e}\right)\right]^{2}},
\end{aligned}
$$

which simplify the expression for the minimization.

$$
0=\sum_{j_{v}^{\prime}, \Lambda^{\prime}} \alpha_{j_{v}, j_{v}^{\prime}}^{\Lambda, \Lambda^{\prime}} \bar{X}_{j_{v}^{\prime}}^{\Lambda^{\prime}}-\beta_{j_{v}}^{\Lambda}
$$

Finally we reach an expression for the fit parameters, CFFs in our case, that implies the inversion of the matrix $\alpha_{j_{v}, j_{v}^{\prime}}^{\Lambda, \Lambda^{\prime}}$ and its subsequent multiplication by $\beta_{j_{v}}^{\Lambda}$. This computation will give us a vector with the different values for each $\bar{X}_{j_{v}}^{\Lambda}$,

$$
\bar{X}_{j_{v}}^{\Lambda}=\sum_{j_{v}^{\prime}, \Lambda^{\prime}}\left[\alpha^{-1}\right]_{j_{v}, j_{v}^{\prime}}^{\Lambda, \Lambda^{\prime}} \beta_{j_{v}^{\prime}}^{\Lambda^{\prime}} .
$$

The covariance matrix for the fitted parameters is

$$
V_{j_{v}, j_{v}^{\prime}}^{\Lambda, \Lambda^{\prime}}=\left[\alpha^{-1}\right]_{j_{v}, j_{v}^{\prime}}^{\Lambda, \Lambda^{\prime}}
$$

After fitting the harmonics $\mathcal{C}_{n}$ to our experimental yields, we extract the experimental cross section for each bin $j_{e}$ (and associated error bars) through the expression [78]

$$
d^{4} \sigma^{E x p}\left(j_{e}\right)=d^{4} \sigma^{F i t}\left(j_{e}\right) \frac{N^{E x p}\left(j_{e}\right)}{N^{M C}\left(j_{e}\right)} .
$$

\subsubsection{Cross sections}

The fit parameters are calculated for each kinematics computing all data available from each run. The fit parameters and their errors are shown along with the photon leptoproduction cross sections. The cross sections are shown as a function of the angle $\varphi_{\gamma}$ for 
every bin in $t$. The contribution to the cross section from every fit parameter along with its kinematic factor is also provided as well as the total contribution from the $\mathrm{BH}$ process. Also, the comparison of the counts of DVCS events from experiment (black dots) and Monte Carlo simulation (red dots) is given next to its corresponding cross section. Table 4.2 contains the values of $t$ comprised in each bin.

\begin{tabular}{c|c|c}
$t_{\min }$ & $t_{\text {max }}$ & $t_{\text {mean }}$ \\
\hline 0 & -0.22 & -0.19 \\
\hline-0.22 & -0.28 & -0.25 \\
\hline-0.28 & -0.34 & -0.31 \\
\hline-0.34 & -0.41 & -0.37 \\
\hline-0.41 & -0.50 & -0.44
\end{tabular}

Table 4.2: Values of $t$ for each bin $\left(G e V^{2}\right)$. 

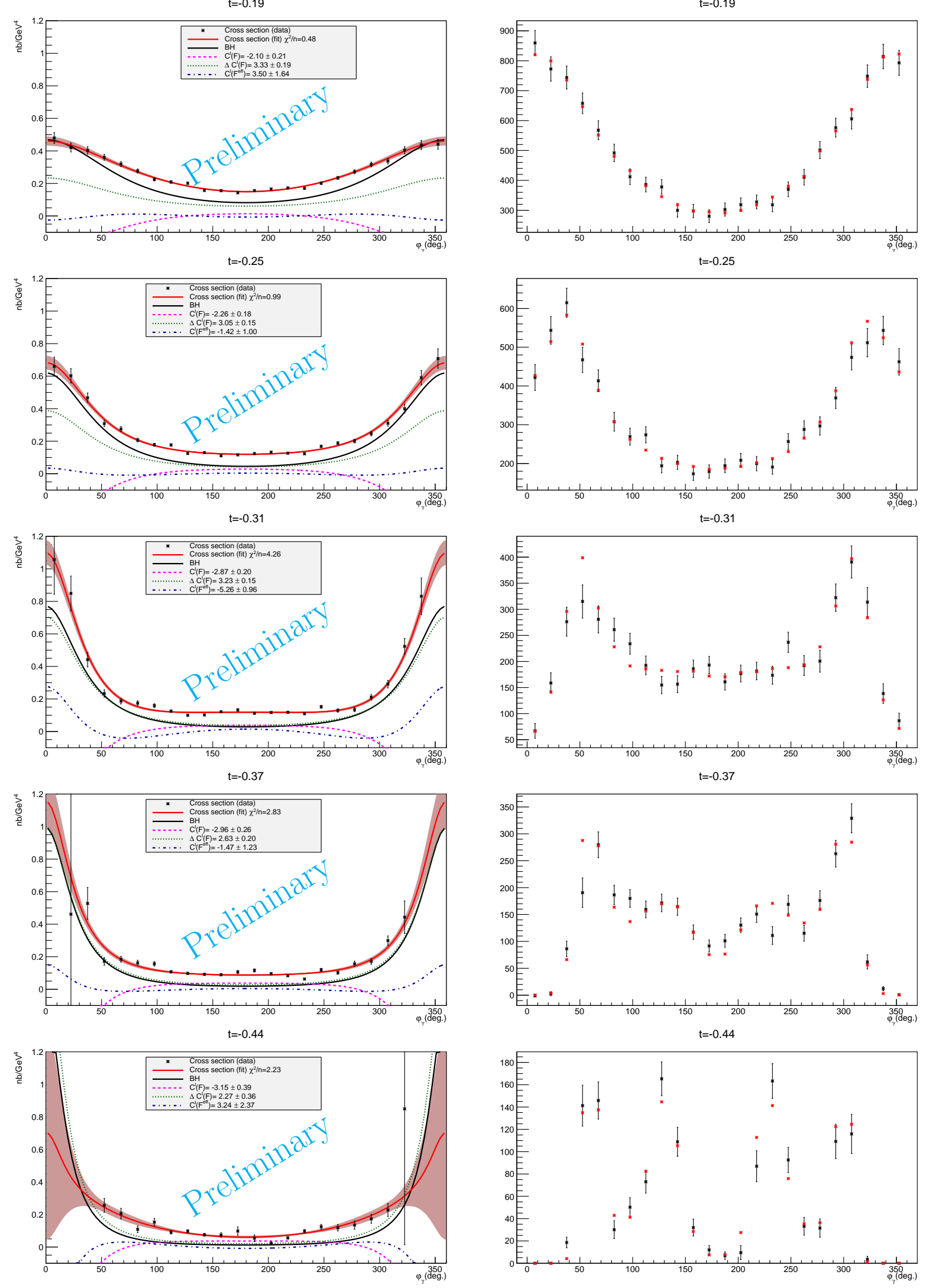

Figure 4.20: Left figures: Preliminary cross sections for Kinematics 1-low.

Right figures: Comparison between the counts of DVCS events from experiment (black dots) and from the Monte Carlo simulation (red dots). No systematic errors considered. 

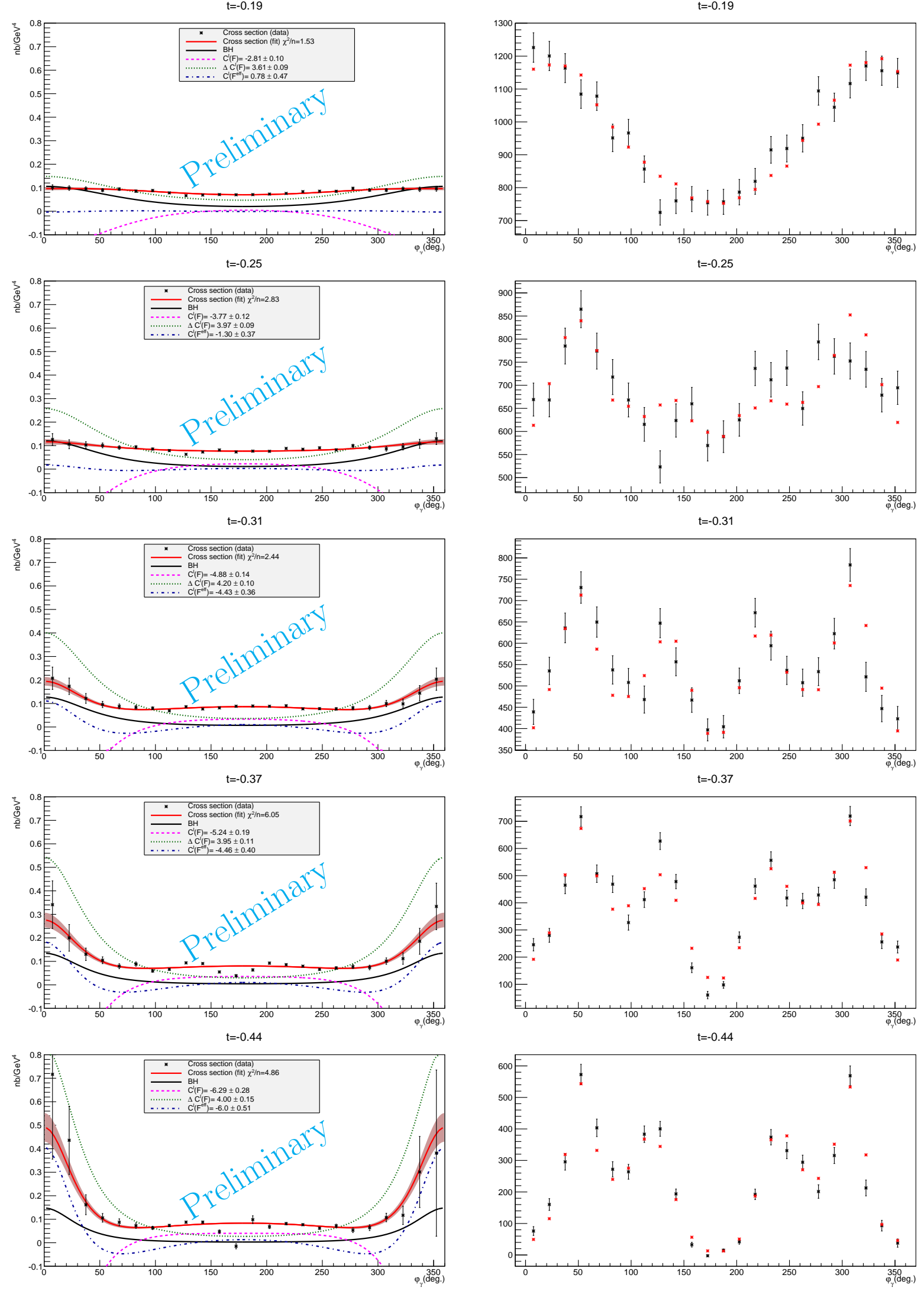

Figure 4.21: Left figures: Preliminary cross sections for Kinematics 1-high.

Right figures: Comparison between the counts of DVCS events from experiment (black dots) and from the Monte Carlo simulation (red dots). No systematic errors considered. 

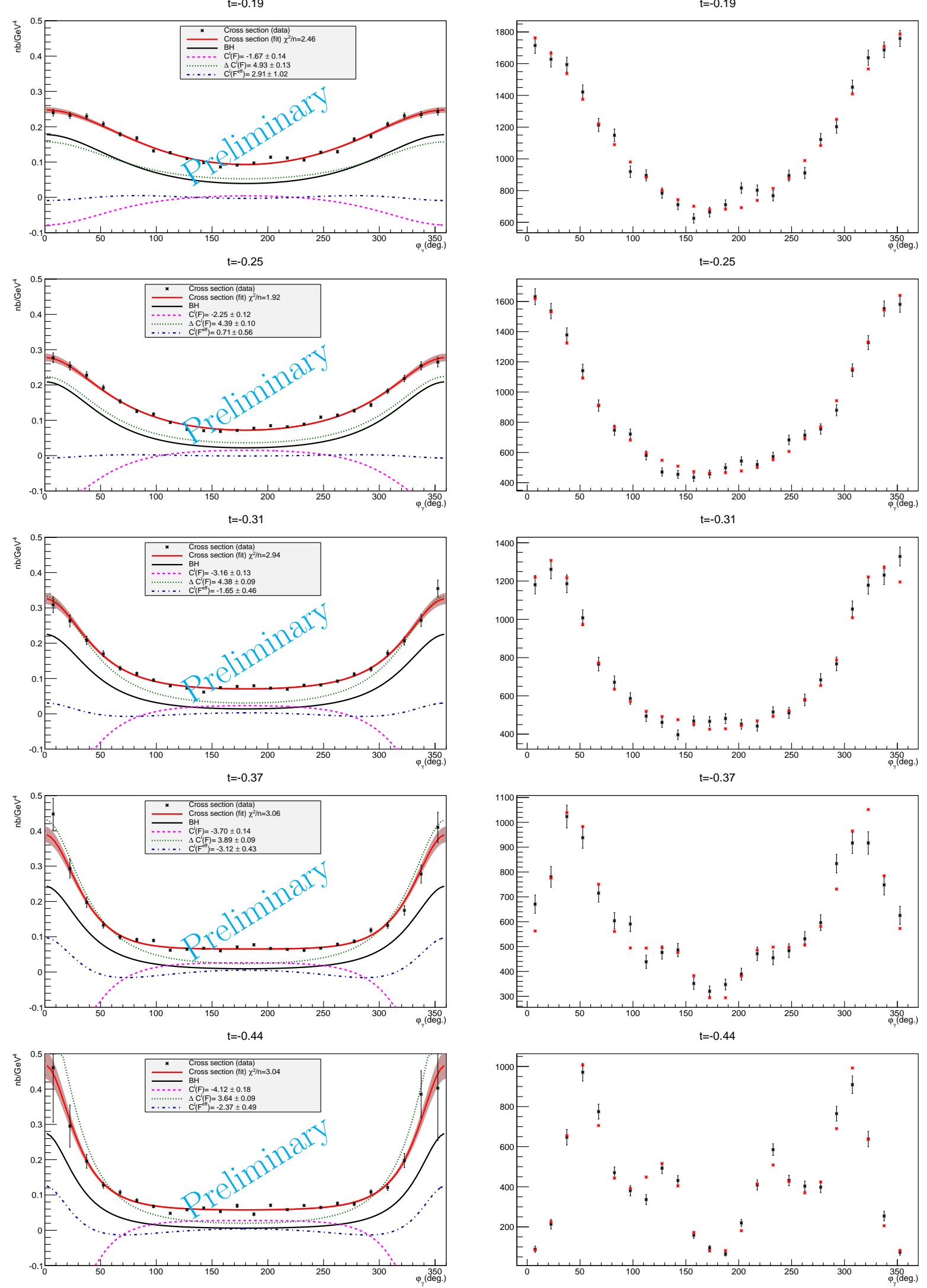

Figure 4.22: Left figures: Preliminary cross sections for Kinematics 2-low.

Right figures: Comparison between the counts of DVCS events from experiment (black dots) and from the Monte Carlo simulation (red dots). No systematic errors considered. 

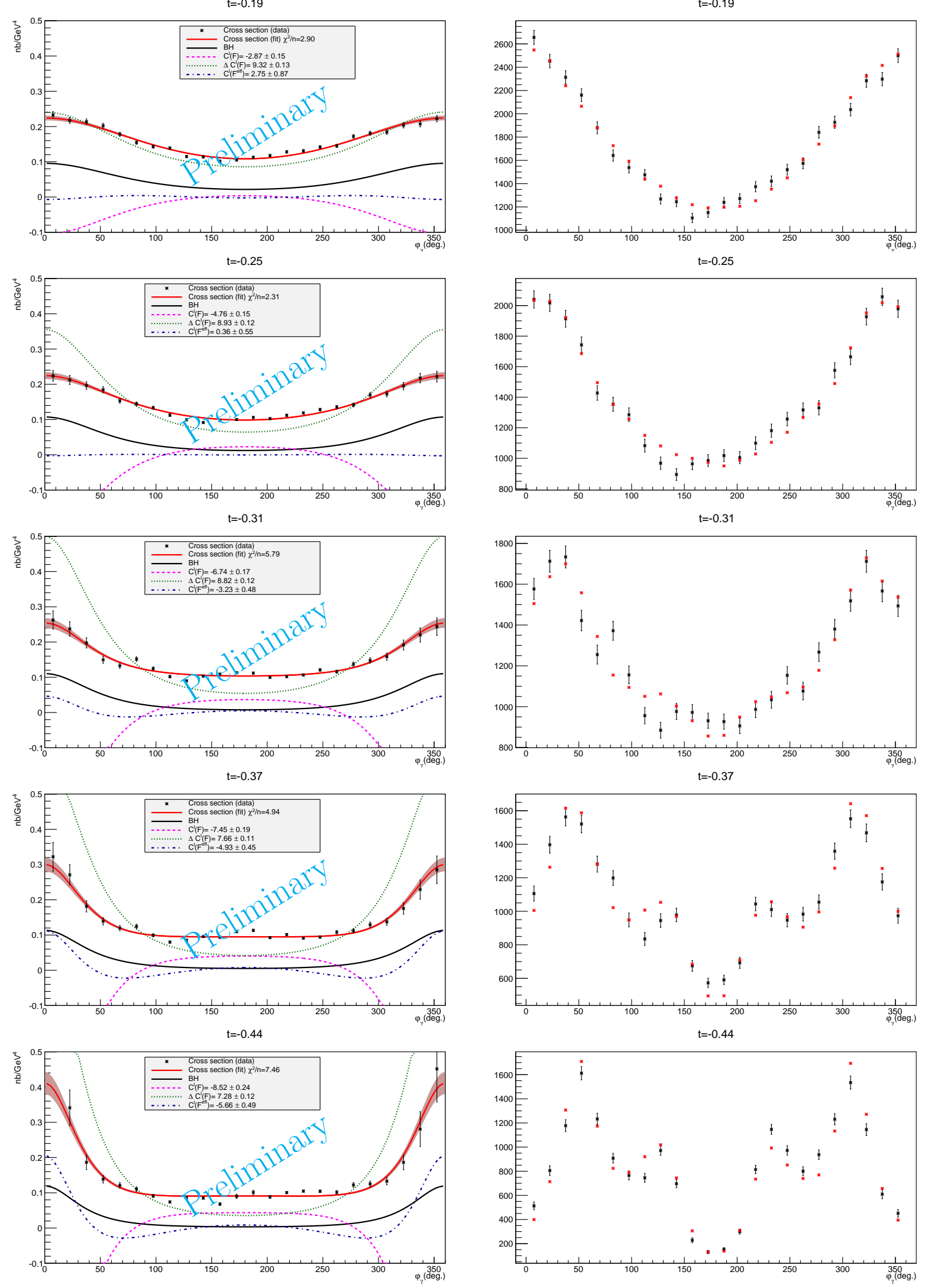

Figure 4.23: Left figures: Preliminary cross sections for Kinematics 2-high.

Right figures: Comparison between the counts of DVCS events from experiment (black dots) and from the Monte Carlo simulation (red dots). No systematic errors considered. 

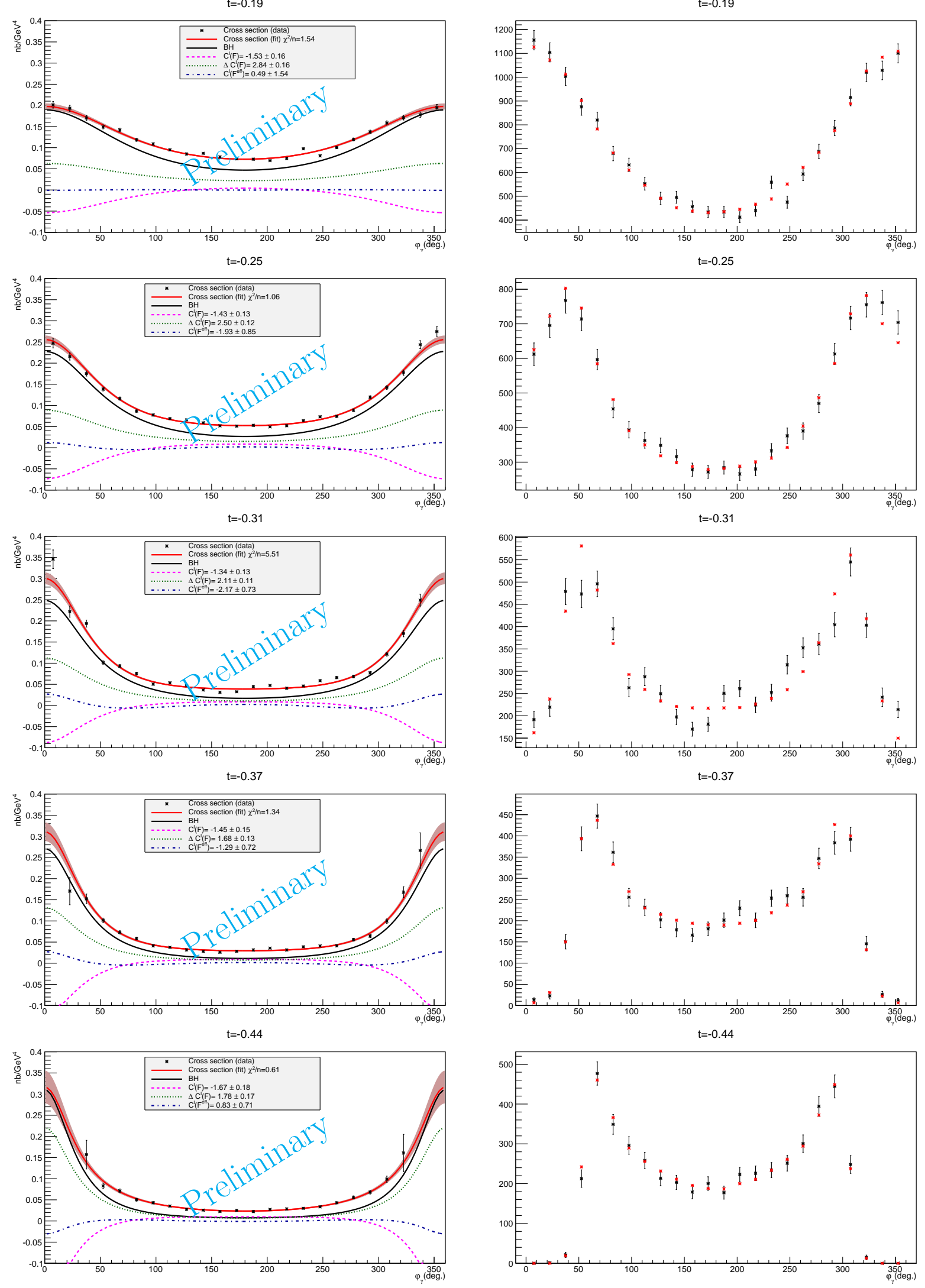

Figure 4.24: Left figures: Preliminary cross sections for Kinematics 3-low.

Right figures: Comparison between the counts of DVCS events from experiment (black dots) and from the Monte Carlo simulation (red dots). No systematic errors considered. 

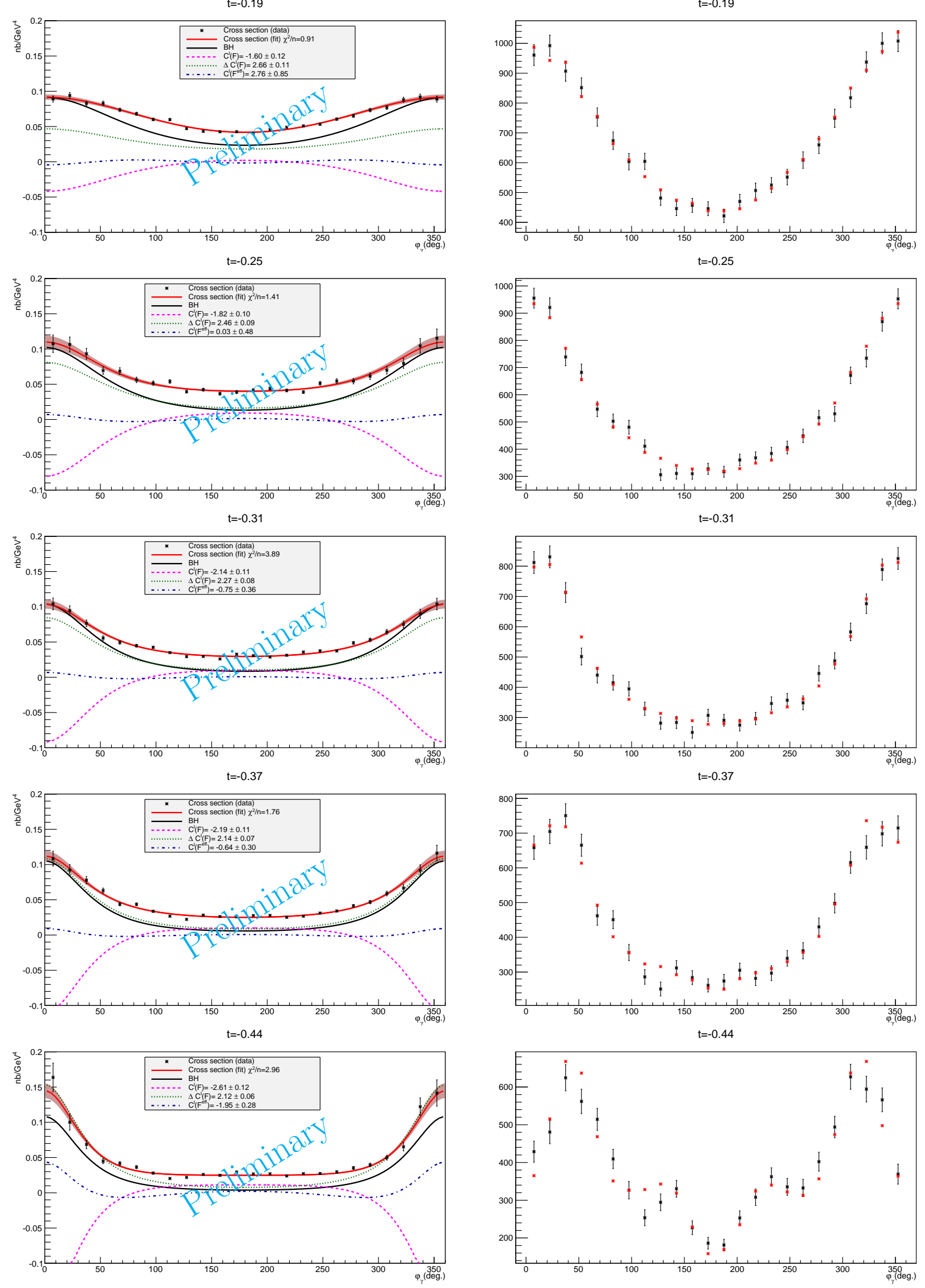

Figure 4.25: Left figures: Preliminary cross sections for Kinematics 3-high.

Right figures: Comparison between the counts of DVCS events from experiment (black dots) and from the Monte Carlo simulation (red dots). No systematic errors considered. 
The results shown in this thesis enclose preliminary cross sections for the different kinematics. We observe a high $\chi^{2}$ in some of the cases, for which we have not found an explanation so far. We believe that reducing reducing the number of $t$-bins to four might increase the accuracy of the fits, decreasing the impact of the acceptance in the borders of the calorimeter (bin with higher $-t$ ). We also observe a deviation of the photon leptoproduction cross section from the pure $\mathrm{BH}$ contribution, which for any $t$ always shows around $180^{\circ}$. We observe this behavior in the results obtained in experiment E00-110 (see Figure 1.15) as well. Although these results do not correspond to any of the studied kinematics in this thesis $\left(E_{b}=5.75 \mathrm{GeV}, \mathcal{Q}^{2}=2.3 \mathrm{GeV}^{2}\right.$ and $\left.t=0.28 \mathrm{GeV}^{2}\right)$, we observe some similarity between the contributions of the $\mathrm{BH}$ and $\mathcal{C}_{\text {unp }}^{\mathcal{I}}(\mathcal{F})$ terms of the second and third bin of kin3-high $\left(E_{b}=5.552 \mathrm{GeV}, \mathcal{Q}^{2}=2 \mathrm{GeV}^{2}\right.$ and $\left.t=0.25,-0.31 \mathrm{GeV}^{2}\right)$, and the contributions found for E00-110. We also find that for the settings with higher beam energies, the deviation for the photon leptoproduction cross section from the pure $\mathrm{BH}$ contribution becomes larger. This deviation appears for angles close to the beam direction $\left(0^{\circ}-360^{\circ}\right)$ as $-t$ increases.

Beyond the computation of the cross sections, the goal of experiment E07-007 is to separate the different contributing amplitudes (eg. BH*DVCS interference from the pure DVCS $^{2}$ term) at all three $\mathcal{Q}^{2}$ values of the previous experiments through a Rosenbluth separation. The results of the experiment will serve to test if factorization in this channel can be obtained at these energies. However, this task has not been addressed during this thesis, due to lack of time. Nevertheless, I will go through the details of the procedure of the Rosenbluth separation in the following lines.

\subsection{Rosenbluth separation}

For the case of the Rosenbluth separation we perform a decomposition of the cross section, similar to the one we performed in chapter 4. Again we include the contribution from the $\mathrm{BH}$ process, but this time the kinematic factors will be different since the decomposition will be done in terms of the $\phi$ dependence. We will extract three different contributions: a term with a $\cos (\phi)$ dependence, another term with a $\cos (2 \phi)$ dependence, and the contribution we are interested in, a $\phi$ independent term. The purpose of this decomposition is to obtain the $\phi$ independent term, related to the DVCS ${ }^{2}$ amplitude, which encloses the contribution from the $\operatorname{CFF} \mathcal{C}^{D V C S}\left(\mathcal{F}, \mathcal{F}^{*}\right)$.

$$
\frac{d^{4} \sigma}{d x d\left|\Delta^{2}\right| d \phi d \mathcal{Q}^{2}}=\Gamma^{G}\left|\mathcal{T}_{B H}\right|^{2}+\Gamma_{\phi}^{1} X_{j_{v}}^{1}+\Gamma_{\phi}^{2} X_{j_{v}}^{2}+\Gamma_{\phi}^{3} X_{j_{v}}^{3} .
$$


We can express the cross section in a compact way as

$$
\begin{aligned}
& d^{4} \sigma\left(x_{v}\right)= \sum_{\Lambda=0}^{3} \Gamma_{\phi}^{\Lambda}\left(x_{v}\right) X_{j_{v}}^{\Lambda}, \\
& \operatorname{with}\left\{\begin{array}{l}
\Gamma_{\phi}^{0}\left(x_{v}\right) X_{j_{v}}^{0}=\Gamma^{G}\left(x_{v}\right)\left|\mathcal{T}_{B H}\right|_{j_{v}}^{2} \\
\Gamma_{\phi}^{1}\left(x_{v}\right) X_{j_{v}}^{1} \rightarrow \phi \text { independent term } \\
\Gamma_{\phi}^{2}\left(x_{v}\right) X_{j_{v}}^{2}=\Gamma_{\phi}^{2}\left(x_{v}\right) \mathcal{C}_{\text {unp }}^{\mathcal{I}}(\mathcal{F})_{j_{v}} \cos (\phi) \\
\Gamma_{\phi}^{3}\left(x_{v}\right) X_{j_{v}}^{3}=\Gamma_{\phi}^{3}\left(x_{v}\right) \mathcal{C}_{u n p}^{\mathcal{I}}\left(\mathcal{F}^{e f f}\right)_{j_{v}} \cos (2 \phi)
\end{array}\right\}
\end{aligned}
$$

In this case, the $X_{j_{v}}^{1}$ term is a combination of the observables $\mathcal{C}_{\text {unp }}^{\mathcal{I}}(\mathcal{F}), \Delta \mathcal{C}_{\text {unp }}^{\mathcal{I}}(\mathcal{F})$ and $\mathcal{C}^{D V C S}\left(\mathcal{F}, \mathcal{F}^{*}\right)$. All these CCFs are accompanied by a $\phi$ independent kinematic factor $\Gamma_{\phi}^{1}\left(x_{v}\right)$ (although this term encloses a $\phi$ dependence in $\Gamma^{\mathcal{I}},(4.20)$ ). Once this term is isolated from the rest, we will use the observables from each pair of kinematics (kinematics high-low, obtained using different beam energies) in order to disentangle the contribution of $\mathcal{C}^{D V C S}\left(\mathcal{F}, \mathcal{F}^{*}\right)$ from the $\phi$ independent term. On the other hand, $X_{j_{v}}^{2}$ and $X_{j_{v}}^{3}$ correspond to the $\mathrm{CFFs} \mathcal{C}_{\text {unp }}^{\mathcal{I}}(\mathcal{F})$ and $\mathcal{C}_{\text {unp }}^{\mathcal{I}}\left(\mathcal{F}^{e f f}\right)$ respectively, and are multiplied by a $\cos (\phi)$ and $\cos (2 \phi)$ factor, again, respectively. The kinematic factors employed in the cross section decomposition read

$\Gamma_{\phi}^{1}=\Gamma^{G} \cdot\left\{\Gamma^{\mathcal{I}}\left[-8(2-y)\left[\frac{(2-y)^{2}}{1-y} K^{2}+\frac{\Delta^{2}}{\mathcal{Q}^{2}}(1-y)\left(2-x_{B}\right)\right]\right]+\Gamma^{D V C S}\left[2\left(2-2 y-y^{2}\right)\right]\right\}$,

$\Gamma_{\phi}^{2}=\Gamma^{G} \cdot \Gamma^{\mathcal{I}}\left\{-8 K\left(2-2 y-y^{2}\right)\right\}$

$\Gamma_{\phi}^{3}=\Gamma^{G} \cdot \Gamma^{\mathcal{I}}\left\{\frac{-16 K^{2}}{2-x_{B}}(2-y)\right\}$

where $\Gamma^{D V C S}$ is the factor multiplying the Fourier decomposition of the DVCS amplitude,

$$
\Gamma^{D V C S}=\frac{1}{y^{2} \mathcal{Q}^{2}}
$$

The process for obtaining the observables $X_{j_{v}}^{1}, X_{j_{v}}^{2}$ and $X_{j_{v}}^{3}$ follows the same path as in chapter 4 , but using the different kinematic factors showed above. Once we have obtained the values of these observables for each bin in $t$ we will proceed to perform the Rosenbluth separation. The $\Gamma_{\phi}^{1}\left(x_{v}\right) X_{j_{v}}^{1}$ term gives us the $\phi$ independent contribution to the cross section, which encloses the $\mathcal{C}^{D V C S}\left(\mathcal{F}, \mathcal{F}^{*}\right)$ CFF. In order to disentangle this CFF from the $\phi$ independent term, we decompose $X_{j_{v}}^{1}$ into the new observables $X_{j_{v}}^{1, a}$ and $X_{j_{v}}^{1, b}$. Here $X_{j_{v}}^{1, a}$ corresponds to $\mathcal{C}^{D V C S}\left(\mathcal{F}, \mathcal{F}^{*}\right)$ and $X_{j_{v}}^{1, b}$ corresponds to a combination of the interference $\mathrm{CFFs}, \mathcal{C}_{\text {unp }}^{\mathcal{I}}(\mathcal{F})$ and $\Delta \mathcal{C}_{\text {unp }}^{\mathcal{I}}(\mathcal{F})$. To perform the separation we will employ the 
$\phi$ independent terms from each pair of kinematics (high-low). Since the dependence with the beam energy is enclosed in the kinematic factors, we assume that $\mathcal{C}^{D V C S}\left(\mathcal{F}, \mathcal{F}^{*}\right)$ will have the same value for each pair of kinematics. With this idea in mind, we will try to fit the $\phi$ independent contribution to the cross section for the two different beam energies, $\Gamma_{\phi, E_{b}}^{1} X_{j_{v}, E_{b}}^{1}$, to a combination of the contributions from the DVCS ${ }^{2}$ and the interference amplitudes, $\Gamma_{\phi, E_{b}}^{1, a} X_{j_{v}}^{1, a}+\Gamma_{\phi, E_{b}}^{1, b} X_{j_{v}}^{1, b}$. The $\phi$ dependence of the kinematic factors adds some complexity to the computation of the $\mathrm{DVCS}^{2} \mathrm{CFF}$. We define a $\chi^{2}$, for each bin in $t$, that we will later minimize in order to extract $X_{j_{v}}^{1, a}$ and $X_{j_{v}}^{1, b}$,

$$
\chi^{2}=\sum_{\phi, E_{b}} \frac{\left[\Gamma_{\phi, E_{b}}^{1} X_{j_{v}, E_{b}}^{1}-\Gamma_{\phi, E_{b}}^{1, a} X_{j_{v}}^{1, a}-\Gamma_{\phi, E_{b}}^{1, b} X_{j_{v}}^{1, b}\right]^{2}}{\left[\sigma^{X_{j_{v}, E_{b}}^{1}}\right]^{2}} .
$$

Here $\sigma^{X_{j_{v}, E_{b}}^{1}}$ is the error associated to each of the two $X_{j_{v}, E_{b}}^{1}$ (one for each of the two kinematics). Note that $X_{j_{v}}^{1, a}$ and $X_{j_{v}}^{1, b}$ do not have a dependence on the beam energy since they will be equal for both kinematics. The idea is to compute these observables by fitting them to the extracted $\phi$ independent terms from the cross sections. The kinematic factors multiplying these two observables come from the factorization of $\Gamma_{\phi, E_{b}}^{1}$ into the $\mathrm{DVCS}^{2}$ and interference contributions. The minimization of the $\chi^{2}$ in terms of these two observables renders the following set of equations

$$
\begin{aligned}
& 0=\frac{\partial \chi^{2}}{\partial X^{1, a} j_{v}}=\sum_{\phi, E_{b}}\left[\Gamma_{\phi, E_{b}}^{1} X_{j_{v}, E_{b}}^{1}-\Gamma_{\phi, E_{b}}^{1, a} X_{j_{v}}^{1, a}-\Gamma_{\phi, E_{b}}^{1, b} X_{j_{e}}^{1, b}\right] \Gamma_{\phi, E_{b}}^{1, a} \\
& 0=\frac{\partial \chi^{2}}{\partial X^{1, b} j_{v}}=\sum_{\phi, E_{b}}\left[\Gamma_{\phi, E_{b}}^{1} X_{j_{v}, E_{b}}^{1}-\Gamma_{\phi, E_{b}}^{1, a} X_{j_{v}}^{1, a}-\Gamma_{\phi, E_{b}}^{1, b} X_{j_{e}}^{1, b}\right] \Gamma_{\phi, E_{b}}^{1, b}
\end{aligned}
$$

Which can be written in the matricial form

$$
\left(\begin{array}{c}
\sum_{\phi, E_{b}} \Gamma_{\phi, E_{b}}^{1} \Gamma_{\phi, E_{b}}^{1, a} X_{j_{v}, E_{b}}^{1} \\
\sum_{\phi, E_{b}} \Gamma_{\phi, E_{b}}^{1} \Gamma_{\phi, E_{b}}^{1, b} X_{j_{v}, E_{b}}^{1}
\end{array}\right)=\left(\begin{array}{cc}
\sum_{\phi, E_{b}} \Gamma_{\phi, E_{b}}^{1, a} \Gamma_{\phi, E_{b}}^{1, a} & \sum_{\phi, E_{b}} \Gamma_{\phi, E_{b}}^{1, a} \Gamma_{\phi, E_{b}}^{1, b} \\
\sum_{\phi, E_{b}} \Gamma_{\phi, E_{b}}^{1, a} \Gamma_{\phi, E_{b}}^{1, b} & \sum_{\phi, E_{b}} \Gamma_{\phi, E_{b}}^{1, b} \Gamma_{\phi, E_{b}}^{1, b}
\end{array}\right)\left(\begin{array}{c}
X_{j_{v}}^{1, a} \\
\\
X_{j_{v}}^{1, b}
\end{array}\right)
$$

By inverting the $2 \times 2$ matrix and multiplying the result by the matrix on the left we would obtain the $\operatorname{DVCS}^{2} \mathrm{CFF}, \mathcal{C}^{D V C S}\left(\mathcal{F}, \mathcal{F}^{*}\right)$, and the contribution from the interference amplitude. Since $X_{j_{v}}^{2}$ is also computed through this process, the contribution from the

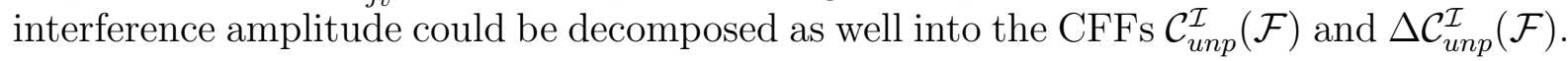





\section{Conclusions}

This thesis focuses on the dedicated EM calorimeter that was used to detect the photon in experiment E07-007 at Jefferson Lab. Although I started my Ph.D. after the data acquisition, I had the chance to visit Jefferson Lab while the experiment was running and observe the calorimeter performance. Experiment E07-007 is enclosed in the second generation of DVCS experiments in Jefferson Lab. This experiment, along with experiment E08-025 [77] builds on the success of the first round of data taking [78], [79]. There is a third generation of DVCS experiments coming up that will take place once the upgrade of the CEBAF facility is operational. The first upcoming DVCS experiment [80] will seek to obtain DVCS cross sections at a wider $\mathcal{Q}^{2}$ coverage, using the increased beam energy (up to $12 \mathrm{GeV}$ ). This will determine with what precision the handbag amplitude dominates (or not) over the higher-twist amplitudes. The amount of DVCS experiments being carried out shows the importance and the value of the information we expect to find from the nucleon structure, and the quark position and momentum within the nucleon.

Regarding the theoretical frame of the photon leptoproduction reaction, I went through the whole computation of the CFFs and the factorization in Fourier harmonics of the cross section, as explained in section 1.4. This was a way of getting a direct idea about the core goal of DVCS experiments, and about our level of understanding of the proton structure at this moment.

The first task I took on when starting this thesis, was the calibration of the calorimeter. It was designed for DVCS experimentation purposes, and it is an upgraded version of the very same device used in previous DVCS experiments. The upgrade was performed with the aim of employing the detector in the experiment E07-007. In order to perform the calibration, I analyzed the stored data from calibration sessions, applied several fiducial cuts that I had to adjust in order to improve the efficiency of the device, and dealt with the issues of block malfunctioning stated in section 3.3.3. The fact that several calibration sessions, using elastic processes, took place during the experiment makes the calibration reliable for any given run. The stability of the coefficients as well as the test using $\pi^{0}$ 
decays confirm this statement. Also, through the elastic calibration data, we computed the values of the energy and angular resolution of the calorimeter, which are satisfactory. A priori, the vertical and horizontal resolutions should be similar. However, we find that the vertical resolution is a bit worse than the horizontal one. One reason for this might be that the vertical resolution of the HRS is worse that the horizontal one $(6 \mathrm{mrad}$ in $x$ and 2 mrad in $y$ ).

After performing the calibration, we were in a position to analyse the data stored for each block of the calorimeter. The first step was to discard runs with known problems that rendered them unfit for the analysis. The sampling of the PMTs current as a response of the arriving particles is something peculiar in the data acquisition system. One could have decided to set an energy threshold in order to select the events as a function of the maximum of the signal. However, due to the high luminosity of the beam in Hall A it is important to be able to analyse events which would have been discarded due to pile-up if we had decided to store data in this way. In the case of the kinematics Kin 3 -high, we found around $8 \%$ of blocks with two pulses. At this stage I implemented the 2-pulse analysis algorithm, and optimized the different parameters showed on Table 3.2, including the study of the different analysing thresholds, in order to increase the precision and speed of the signal analysing process. I also applied the time correction for each block on each run of the stored data, by studying the time of arrival of the stored signals, as explained in section 3.1.5.

The clustering algorithm proves to be a powerful tool for computing impact points in the surface of the calorimeter as well as for discerning multiple impacts from different particles. My work involving the clustering algorithm includes its modification in order to store data from two-cluster events as well as the study of the clustering threshold. This threshold helps us discriminate the events using the number of formed clusters, although a multicluster correction is necessary. Besides providing a way to obtain the kinematic variables of the hitting particles, essential for the cross section computation, it allowed us to perform the subtraction of the $\pi^{0}$ background without having to compute the cross section for this kind of contamination.

The next step in calculating the photon leptoproduction cross sections was to subtract the background from the data. Here I performed the $\pi^{0}$ and the accidental subtraction. For the $\pi^{0}$ subtraction, I had to implement an algorithm that simulated the surface of the electromagnetic calorimeter. Then, I had to perform the randomization of the $\pi^{0}$ decays, taking into account the kinematics and geometry of each decay, such as performing a correction for the vertex impact point and including in the computation the fact that most of the particles energy is deposited at a $7 \mathrm{~cm}$ depth inside the calorimeter, in the direction of the particle's line of flight. For the case of the accidentals subtraction, I extracted the sample of accidental detections for each run and then, extracted each acci- 
dental event from the corresponding experimental bin of DVCS events, according to its kinematics. As stated before, a multicluster correction was needed to take into account the DVCS detections found in two-cluster events. The correction included the analysis of all two-cluster events, following the same steps employed in the analysis of one-cluster events.

The computation of the cross sections were obtained using a Monte Carlo simulation of the experiment. The simulation was employed to fit the data and obtain the different contributions to the cross section. I computed the cross sections by following the steps shown in section 4.8. All fiducial cuts were applied to the simulation data before performing the extraction of observables. Then I introduced the coefficients shown in Appendix $\mathrm{C}$ into the computation, and performed the binning of the cross section in the $t$ and $\varphi_{\gamma}$ variables. After going through all these tasks I finally obtained the preliminary photon leptoproduction cross section for every kinematic employed in the experiment, along with the contributions from BH, $\mathcal{C}_{\text {unp }}^{\mathcal{I}}(\mathcal{F}), \Delta \mathcal{C}_{\text {unp }}^{\mathcal{I}}(\mathcal{F})$, and $\mathcal{C}_{\text {unp }}^{\mathcal{I}}\left(\mathcal{F}^{\text {eff }}\right)$, as shown in Figures 4.204.25 .

During the last weeks of my thesis I started to perform the Rosenbluth Separation in order to obtain the contribution of the $\mathcal{C}_{\text {unp }}^{D V C S}\left(\mathcal{F}, \mathcal{F}^{*}\right)$ term. However, the computation of this term is not fully implemented and it remains as work to do. Also, the different contributions from systematic errors have yet to be included in the computation of the errors of the cross sections. A further study of the systematic error created by the cut in the "Missing mass" has to be performed. This will include the study of the error in the different kinematics (besides kin3-high), and applying a different cut in different areas of the calorimeter, depending on the resolution in those areas. Finally, we expect to obtain the polarized cross sections, employing the available data on the beam polarization. 

$\prod_{\text {Appendix }}$

\section{Light cone coordinates}

To derive the relevant distances in deeply inelastic scattering, it proves useful to switch to a reference frame where the target proton is at rest and the virtual photons three-momentum points in the direction opposite to the z-axis. Then the virtual photon 4-vector reads [16]:

$$
q^{\mu}=\left(\frac{\mathcal{Q}^{2}}{2 M x_{B}}, 0,0,-\frac{\mathcal{Q}^{2}}{2 M x_{B}} \sqrt{1+4 M^{2} x_{B}^{2} / \mathcal{Q}^{2}}\right) .
$$

We can express $q$ in light-cone coordinates as:

$$
q^{\mu}=q^{+} \tilde{p}^{\mu}+q^{-} n^{\mu}+\vec{q}_{\perp}^{\mu}, \quad q^{+}=q^{0}+q^{3}, \quad q^{-}=q^{0}-q^{3},
$$

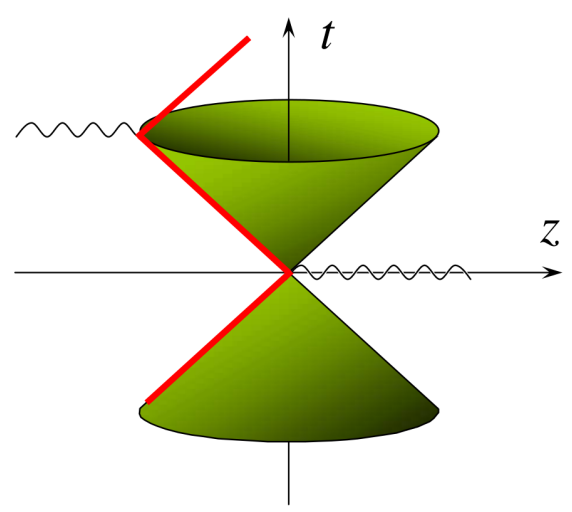

Figure A.1: Light cone: space-time trajectory of the struck quark in the hadron.

and $\tilde{p}$ and $n$ as the Sudakov vectors defined by: 


$$
\tilde{p}=\frac{1}{\sqrt{2}}\left(\begin{array}{c}
1 \\
0 \\
0 \\
1
\end{array}\right), \quad n=\frac{1}{\sqrt{2}}\left(\begin{array}{c}
1 \\
0 \\
0 \\
-1
\end{array}\right)
$$

When $\mathcal{Q}^{2}$ is large, the light-cone components of the momentum transfer can be approximated by:

$$
q^{-} \sim \mathcal{Q}^{2} /\left(M x_{B}\right), \quad \quad q^{+} \sim M x_{B} .
$$

The integrand in (1.13) is an oscillatory function and thus gives vanishing result unless the distances involved are

$$
z^{-} \sim 1 /\left(M x_{B}\right), \quad \quad z^{+} \sim M x_{B} / \mathcal{Q}^{2} .
$$

Causality ensures $z^{2}>0$, and so $z_{\perp}<2 z^{+} z^{-} \sim 1 / \mathcal{Q}^{2}$. Therefore, the only region which contributes to the integral is close to the light-cone $z^{2} \approx 0$. The light-cone distance dominance in scattering amplitudes legitimates the application of powerful methods of perturbative QCD. 
Appendix

\section{DVCS kinematics}

In the target rest frame in which the z-axis is directed opposite to the momentum of the space-like virtual photon (see figure 1.13), we have for the initial proton and virtual photon four-momenta [16],

$$
p^{\mu}=\left(M_{N}, 0,0,0\right), \quad q^{\mu}=\left(w, 0,0,-q^{z}\right),
$$

where the photon energy and the z-component of its three-momentum are expressed as:

$$
w=\frac{\mathcal{Q}}{\varepsilon}, \quad q^{z}=\frac{\mathcal{Q}}{\varepsilon} \sqrt{1+\varepsilon^{2}} .
$$

The outgoing nucleon four-momentum in the target rest frame has the components:

$$
p^{\prime \mu}=\left(E^{\prime}, \mathbf{p}^{\prime}\right), \quad E^{\prime}=M_{N}-\frac{\Delta^{2}}{2 M_{N}}, \quad\left|\mathbf{p}^{\prime}\right|=\sqrt{-\Delta^{2}\left(1-\Delta^{2} /\left(4 M_{N}^{2}\right)\right)} .
$$

and the scattering angle of the recoiled nucleon is:

$$
\cos \left(\theta_{N}\right)=-\frac{\varepsilon^{2}\left(\mathcal{Q}^{2}-\Delta^{2}\right)-2 x_{B} \Delta^{2}}{4 x_{B} M_{N}\left|\mathbf{p}^{\prime}\right| \sqrt{1+\varepsilon^{2}}} .
$$

The incoming electron four-momentum,

$$
k^{\mu}=\left(E, k^{x}, 0, k^{z}\right)=E\left(1, \sin \left(\theta_{e}\right), 0, \cos \left(\theta_{e}\right)\right), \quad E=\frac{\mathcal{Q}}{y \varepsilon}, \quad \cos \left(\theta_{e}\right)=-\frac{1+y \varepsilon^{2} / 2}{\sqrt{1+\varepsilon^{2}}},
$$

depends on the variable proportional to the lepton energy loss,

$$
y=\frac{p \cdot q}{p \cdot k}, \quad q=k-k^{\prime} .
$$

Notice that from the relation 


$$
\sin \left(\theta_{e}\right)=\frac{\varepsilon \sqrt{1-y-y^{2} \varepsilon^{2} / 4}}{\sqrt{1+\varepsilon^{2}}}
$$

it is clear that for large momentum transfer from the lepton to the target, the lepton beam experiences forward scattering with almost no deflection as $\theta_{e} \rightarrow 0$ for $\mathcal{Q}^{2} \gg M_{N}$. Finally, the four-vector of the real photon is given by:

$$
q^{\prime \mu}=\left(w^{\prime}, \mathbf{v} w^{\prime}\right),
$$

with momentum components and the scattering angle being:

$$
w^{\prime}=\frac{\mathcal{Q}}{\varepsilon}+\frac{\Delta^{2}}{2 M_{N}}, \quad|\mathbf{v}|=1, \quad \cos \left(\theta_{\gamma}\right)=-\frac{\varepsilon^{2}\left(\mathcal{Q}^{2}-\Delta^{2}\right)+2 \mathcal{Q} w^{\prime}}{2 \mathcal{Q} w^{\prime} \sqrt{1+\varepsilon^{2}}} .
$$

There is an important difference between the interference term and the squared DVCS amplitude. The former has a contaminating $\phi$-dependence due to the lepton $\mathrm{BH}$ propagators,

$$
\begin{aligned}
& \mathcal{Q}^{2} \mathcal{P}_{1}=\left(k-q_{2}\right)^{2}=\mathcal{Q}^{2}+2 k \cdot \Delta, \\
& \mathcal{Q}^{2} \mathcal{P}_{2}=(k-\Delta)^{2}=\Delta^{2}-2 k \cdot \Delta .
\end{aligned}
$$

where

$$
k \cdot \Delta=-\frac{\mathcal{Q}^{2}}{2 y\left(1+\varepsilon^{2}\right)}\left\{1+2 K \cos (\phi)-\frac{\Delta^{2}}{\mathcal{Q}^{2}}\left(1-x_{B}(2-y)+\frac{y \varepsilon^{2}}{2}\right)+\frac{y \varepsilon^{2}}{2}\right\} .
$$

The $1 / \mathcal{Q}$-power suppressed kinematical factor $K$ appearing here also shows up in the Fourier series,

$$
K \equiv \frac{1}{2 \mathcal{Q}^{2}} \sqrt{-\left(1-y-y^{2} \varepsilon^{2} / 4\right)\left(4 x_{B}\left(1-x_{B}\right)+\varepsilon^{2}\right)\left(\Delta^{2}-\Delta_{\min }^{2}\right)\left(\Delta^{2}-\Delta_{\max }^{2}\right)} .
$$

We expressed $\mathrm{K}$ in terms of the maximal and minimal momentum transfer in the t-channel,

$$
\Delta_{\text {min }, \text { max }}^{2}=\frac{1}{4 x_{B}\left(1-x_{B}\right) \varepsilon^{2}}\left\{2\left(1-x_{B}\right) \mathcal{Q}^{2}+\varepsilon^{2} \mathcal{Q}^{2} \mp 2 \sqrt{1+\varepsilon^{2}}\left(1-x_{B}\right) \mathcal{Q}^{2}\right\},
$$

with - $(+)$ corresponding to $\Delta_{\min }^{2}\left(\Delta_{\max }^{2}\right)$. It vanishes at the kinematical boundary $\Delta^{2}=$ $\Delta_{\text {min }}^{2}$, determined by the minimal value

$$
-\Delta_{\min }^{2} \approx \frac{M_{N}^{2} x_{B}^{2}}{1-x_{B}+x_{B} M_{N}^{2} / \mathcal{Q}^{2}} .
$$




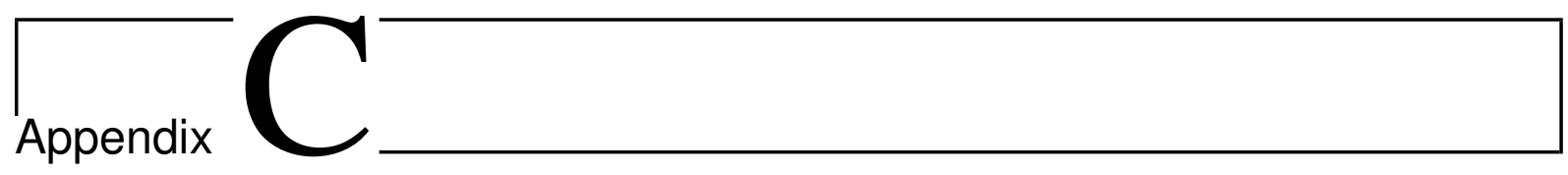

\section{Coefficients and Compton form factors}

We list the coefficient of the Fourier decomposition of the photon leptoproduction cross section as well as the Compton Form Factors they refer to [16]. We only include the coefficients for the unpolarized target.

\section{C.1 Bethe-Heitler coefficients}

$$
\begin{aligned}
c_{0, \text { unp }}^{B H} & =8 K^{2}\left\{\left(2+3 \epsilon^{2}\right) \frac{\mathcal{Q}^{2}}{\Delta^{2}}\left(F_{1}^{2}-\frac{\Delta^{2}}{4 M^{2}} F_{2}^{2}\right)+2 x_{B}^{2}\left(F_{1}+F_{2}\right)^{2}\right\} \\
& +(2-y)^{2}\left\{\left(2+\epsilon^{2}\right)\left[\frac{4 x_{B}^{2} M^{2}}{\Delta^{2}}\left(1+\frac{\Delta^{2}}{\mathcal{Q}^{2}}\right)^{2}+4\left(1-x_{B}\right)\left(1+x_{B} \frac{\Delta^{2}}{\mathcal{Q}^{2}}\right)\right]\left(F_{1}^{2}-\frac{\Delta^{2}}{4 M^{2}} F_{2}^{2}\right)\right. \\
& \left.+4 x_{B}^{2}\left[x_{B}+\left(1-x_{B}+\frac{\epsilon^{2}}{2}\right)\left(1-\frac{\Delta^{2}}{\mathcal{Q}^{2}}\right)^{2}-x_{B}\left(1-2 x_{B}\right) \frac{\Delta^{4}}{\mathcal{Q}^{4}}\right]\left(F_{1}+F_{2}\right)^{2}\right\} \\
& +8\left(1+\epsilon^{2}\right)\left(1-y-\frac{\epsilon^{2} y^{2}}{4}\right)\left\{2 \epsilon^{2}\left(1-\frac{\Delta^{2}}{4 M^{2}}\right)\left(F_{1}^{2}-\frac{\Delta^{2}}{4 M^{2}} F_{2}^{2}\right)-x_{B}^{2}\left(1-\frac{\Delta^{2}}{\mathcal{Q}^{2}}\right)^{2}\left(F_{1}+F_{2}\right)^{2}\right\}
\end{aligned}
$$

$$
c_{1, \text { unp }}^{B H}=8 K(2-y)\left\{\left(\frac{4 x_{B}^{2} M^{2}}{\Delta^{2}}-2 x_{B}-\epsilon^{2}\right)\left(F_{1}^{2}-\frac{\Delta^{2}}{4 M^{2}} F_{2}^{2}\right)+2 x_{B}^{2}\left(1-\left(1-2 x_{B}\right) \frac{\Delta^{2}}{\mathcal{Q}^{2}}\right)\left(F_{1}+F_{2}\right)^{2}\right\}
$$

$$
c_{2, u n p}^{B H}=8 x_{B}^{2} K^{2}\left\{\frac{4 M^{2}}{\Delta^{2}}\left(F_{1}^{2}-\frac{\Delta^{2}}{4 M^{2}} F_{2}^{2}\right)+2\left(F_{1}+F_{2}\right)^{2}\right\}
$$




\section{C.2 DVCS coefficients}

$$
\begin{aligned}
& c_{0, \text { unp }}^{D V C S}=2\left(2-2 y-y^{2}\right) \mathcal{C}_{\text {unp }}^{D V C S}\left(\mathcal{F}, \mathcal{F}^{*}\right) \\
& \left\{\begin{array}{c}
c_{1, \text { unp }}^{D V C S} \\
s_{1, \text { unp }}^{D V C S}
\end{array}\right\}=\frac{8 K}{2-x_{B}}\left\{\begin{array}{c}
2-y \\
-\lambda y
\end{array}\right\}\left\{\begin{array}{c}
\Re e \\
\Im m
\end{array}\right\} \mathcal{C}_{\text {unp }}^{D V C S}\left(\mathcal{F}^{e f f}, \mathcal{F}^{*}\right) \\
& c_{2, \text { unp }}^{D V C S}=-\frac{4 \mathcal{Q}^{2} K^{2}}{M^{2}\left(2-x_{B}\right)} \Re e \mathcal{C}_{T, \text { unp }}^{D V C S}\left(\mathcal{F}_{T}, \mathcal{F}^{*}\right)
\end{aligned}
$$

\section{C.3 Interference coefficients}

$$
\begin{aligned}
& c_{0, \text { unp }}^{\mathcal{I}}=-8(2-y) \Re e\left\{\frac{(2-y)^{2}}{1-y} K^{2} \mathcal{C}_{\text {unp }}^{\mathcal{I}}(\mathcal{F})+\frac{\Delta^{2}}{\mathcal{Q}^{2}}(1-y)\left(2-x_{B}\right)\left(\mathcal{C}_{\text {unp }}^{\mathcal{I}}+\Delta \mathcal{C}_{\text {unp }}^{\mathcal{I}}\right)(\mathcal{F})\right\} \\
& \left\{\begin{array}{c}
c_{1, \text { unp }}^{\mathcal{I}} \\
s_{1, \text { unp }}^{\mathcal{I}}
\end{array}\right\}=8 K\left\{\begin{array}{c}
-\left(2-2 y-y^{2}\right) \\
\lambda y(2-y)
\end{array}\right\}\left\{\begin{array}{c}
\Re e \\
\Im m
\end{array}\right\} \mathcal{C}_{\text {unp }}^{\mathcal{I}}(\mathcal{F}) \\
& \left\{\begin{array}{c}
c_{2, \text { unp }}^{\mathcal{I}} \\
s_{2, \text { unp }}^{\mathcal{I}}
\end{array}\right\}=\frac{16 K^{2}}{2-x_{B}}\left\{\begin{array}{c}
-(2-y) \\
\lambda y
\end{array}\right\}\left\{\begin{array}{c}
\Re e \\
\Im m
\end{array}\right\} \mathcal{C}_{\text {unp }}^{\mathcal{I}}\left(\mathcal{F}^{\text {eff }}\right) \\
& c_{3, \text { unp }}^{\mathcal{I}}=-\frac{8 \mathcal{Q}^{2} K^{3}}{M^{2}\left(2-x_{B}\right)^{2}} \Re e \mathcal{C}_{T, \text { unp }}^{\mathcal{I}}\left(\mathcal{F}_{T}\right)
\end{aligned}
$$

\section{C.4 Compton form factors}

$$
\begin{gathered}
\mathcal{C}_{\text {unp }}^{D V C S}\left(\mathcal{F}, \mathcal{F}^{*}\right)=\frac{1}{\left(2-x_{B}\right)^{2}} \\
\left\{4\left(1-x_{B}\right)\left(\mathcal{H} \mathcal{H}^{*}+\widetilde{\mathcal{H}} \widetilde{\mathcal{H}}^{*}\right)-x_{B}^{2}\left(\mathcal{H} \mathcal{E}^{*}+\mathcal{E} \mathcal{H}^{*}+\widetilde{\mathcal{H}} \widetilde{\mathcal{E}}^{*}+\widetilde{\mathcal{E}} \widetilde{\mathcal{H}}^{*}\right)\right. \\
\left.-\left(x_{B}^{2}+\left(2-x_{B}\right)^{2} \frac{\Delta^{2}}{4 M^{2}}\right) \mathcal{E} \mathcal{E}^{*}-x_{B}^{2} \frac{\Delta^{2}}{4 M^{2}} \widetilde{\mathcal{E}} \widetilde{\mathcal{E}}^{*}\right\}
\end{gathered}
$$$$
\mathcal{C}_{\text {unp }}^{\mathcal{I}}(\mathcal{F})=F_{1} \mathcal{H}+\frac{x_{B}}{2-x_{B}}\left(F_{1}+F_{2}\right) \widetilde{\mathcal{H}}-\frac{\Delta^{2}}{4 M^{2}} F_{2} \mathcal{E}
$$ 


$$
\begin{aligned}
\Delta \mathcal{C}_{\text {unp }}^{\mathcal{I}}(\mathcal{F})= & -\frac{x_{B}}{2-x_{B}}\left(F_{1}+F_{2}\right)\left\{\frac{x_{B}}{2-x_{B}}(\mathcal{H}+\mathcal{E})+\widetilde{\mathcal{H}}\right\} \\
\mathcal{C}_{T, \text { unp }}^{D V C S}\left(\mathcal{F}_{T}\right)= & \frac{1}{\left(2-x_{B}\right)^{2}}\left\{\mathcal{H}_{T}\left[\left(2-x_{B}\right) \mathcal{E}^{*}-x_{B} \widetilde{\mathcal{E}}^{*}\right]-2\left(2-x_{B}\right) \widetilde{\mathcal{H}}_{T}\left[\mathcal{H}^{*}+\frac{\Delta^{2}}{4 M^{2}} \mathcal{E}^{*}\right]\right\} \\
& +\frac{1}{\left(2-x_{B}\right)^{2}}\left\{-\mathcal{E}_{T}\left[\left(2-x_{B}\right) \mathcal{H}^{*}-x_{B} \widetilde{\mathcal{H}}^{*}\right]+\widetilde{\mathcal{E}}_{T}\left[x_{B}\left(\mathcal{H}^{*}+\mathcal{E}^{*}\right)-\left(2-x_{B}\right) \widetilde{\mathcal{H}}^{*}\right]\right\}
\end{aligned}
$$

$\mathcal{C}_{T, \text { unp }}^{\mathcal{I}}\left(\mathcal{F}_{T}\right)=-F_{2} \mathcal{H}_{T}+2\left(F_{1}-\frac{\Delta^{2}}{4 M^{2}} F_{2}\right) \widetilde{\mathcal{H}}_{T}+F_{1} \mathcal{E}_{T}$

where $\left(\mathcal{F}_{T}\right)$ indicates gluon transversity is involved and $\mathcal{F}^{e f f}$ is defined by,

$$
\mathcal{F}^{e f f}=-\frac{2 \xi}{1+\xi} \mathcal{F}+2 \xi\left(\mathcal{F}_{3}^{+}-\mathcal{F}_{3}^{-}\right)
$$





\section{Bibliography}

[1] R. Frisch, O. Stern, Magnetic Deviation of Hydrogen Molecules and the Magnetic Moment of the Proton. I, Z. Phys. 85 (1933) 4.

[2] Murray Gell-Mann, A schematic model of baryons and mesons, Physics Letters. Vol. 8 No. 3 (1964): 214-215.

[3] Robert Hofstadter, The Electron Scattering Method and its Application to the Structure of Nuclei and Nucleons, Nobel Lectures, Physics 1942-1962, pp. 560-581, Elsevier Pub. Co., Amsterdam-London-New York (1961).

[4] SLAC-MIT-CIT Collaboration, Proposals for Initial Electron Scattering Experiments Using the SLAC Spectrometer Facilities, Stanford Linear Accelerator Center Proposal No.4 (1966).

[5] M. Breidenbach, J. I. Friedman, H. W. Kendall, Observed behaviour of highly inelastic electron-proton scattering, SLAC-PUB-650, August (1969).

[6] R. Brandelik et al., (TASSO collaboration), Evidence for Planar Events in e+e- Annihilation at High Energies, Phys. Lett. B 86: 243249 (1979).

[7] D. J. Gross and F. Wilczek, Phys. Rev. Left.30, 1343 (1973); H. D. Politzer, Phys. Rev. Left.30, 1346 (1973).

[8] http://hallaweb.jlab.org/experiment/DVCS/.

[9] https://www.jlab.org/.

[10] Carlos Muñoz Camacho et al. Complete Separation of Deeply Virtual Photon and $\pi^{0}$ Electroproduction Observables of Unpolarized Protons, Proposal for Jefferson Lab PAC 31, E07-007 (2006).

[11] H. Goldstein, C. Poole, J. Safko, Classical Mechanics (third ed.), Adison Wesley, ISBN 0-201-65702-3 (2002). 
[12] F. Halzen and A. D. Martin, Quarks \& leptons (John Wiley \& sons Inc., New York) (1984).

[13] Richard E. Taylor, Rev. Mod. Phys. 63, 573-595 (1991).

[14] Robert L. Jaffe. Spin, Twist and Hadron Structure in Deep Inelastic Processes, Erice (1995) arXiv:hep-ph/9602236v1.

[15] A.V. Radyushkin, Generalized Parton Distributions, Shifman, M. (ed.): At the frontier of particle physics, vol. 2 1037-1099 (2000) 113-116. arXiv:hep-ph/0101225v1.

[16] A.V. Belitsky, A.V. Radyushkin, Unraveling hadron structure with generalized parton distributions, Phys.Rept.418:1-387 (2005). arXiv:hep-ph/0504030v3.

[17] X. Ji, W. Melnitchouk, X. Song, Study of off-forward parton distributions, Phys. Rev. D 56, 5511 (1997).

[18] D. Müller, D. Robaschik, B. Geyer, F.M. Dittes, J. Horejsi, Fortsch. Phys. 42 (1994) 101.

[19] X. Ji, Phys. Rev. Lett. 78 (1997) 610.

[20] A.V. Radyushkin, Phys. Lett. B 380 (1996) 417.

[21] A.V. Radyushkin, Phys. Lett. B 385 (1996) 333.

[22] X. Ji, Phys. Rev. D 55 (1997) 7114.

[23] A.V. Radyushkin, Phys. Rev. D 56 (1997) 5524.

[24] M. Diehl, Phys. Rept. 388 (2003) 41.

[25] A.V. Radyushkin, Generalized parton distributions, in At the Frontier of Particle Physics. Handbook of QCD, Ed. M. Shifman, vol. 2 (World Scientific, 2001) p. 1037; hep-ph/0101225.

[26] K. Goeke, M.V. Polyakov, M. Vanderhaeghen, Prog. Part. Nucl. Phys. 47 (2001) 401.

[27] X. Ji, J. Phys. G 24 (1998) 1181.

[28] X. Ji, Generalized Parton Distributions, Annu. Rev. Nucl. Part. Sci. (2004). 54:41350.

[29] S. Scopetta, V. Vento, Generalized parton distributions in constituent quark models, Eur. Phys. J. A 16 (2003) 527-535.

[30] S. Scopetta, V. Vento, Generalized parton distributions and composite constituent quarks, Phys. Res. D 69 (2004) 094004. 
[31] S. Noguera, S. Scopetta, Relativity and constituent quark structure in model calculations of parton distributions, Phys. Rev. D 70 (2004) 094018.

[32] X. Ji, Gauge-Invariant Decomposition of Nucleon Spin, Phys. Rev. Lett. 78, 610613 (1997).

[33] Michel Guidal, Herve Moutarde, Marc Vanderhaeghen, Generalized Parton Distributions in the valence region from Deeply Virtual Compton Scattering, arXiv:1303.6600v3 (2013).

[34] Claude Itzykson (Author), Jean-Bernard Zuber, Quantum Field Theory (McGrawHill international Book Company), ISBN 0-07-032071-3 (1980).

[35] A.V. Belitsky, D. Müller, A. Kirchner, Theory of deeply virtual Compton scattering on the nucleon, Nucl.Phys. B629 (2002) 323-392. arXiv:hep-ph/0112108v2.

[36] M. Mazouz, A. Ben Fredj, L. Ghedira, Measurement of the Deeply Virtual Compton Scattering cross-section off the neutron, Proposal for Jefferson Lab PAC 33, E08-025 (2008).

[37] C. Muñoz et al., Scaling Tests of the Cross Section for Deeply Virtual Compton Scattering, Phys.Rev.Lett.97:262002,(2006).

[38] M. Vanderhaeghen, P. A. M. Guichon, and M. Guidal, Phys. Rev. D60, 094017 (1999), hep-ph/9905372.

[39] K. Goeke, M. V. Polyakov, and M. Vanderhaeghen, Prog. Part. Nucl. Phys. 47, 401 (2001), hep-ph/0106012.

[40] M. Guidal, M. V. Polyakov, A. V. Radyushkin, and M. Vanderhaeghen, Phys. Rev. D72, 054013 (2005), hep-ph/0410251.

[41] Christoph W. Leemann, David R. Douglas, and Geoffrey A. Krafft. The continuous electron beam accelerator facility: CEBAF at the Jefferson Laboratory. Ann.Rev.Nucl.Part.Sci. 51 (2001) 413-450.

[42] https://www.jlab.org/experimental-hallD.

[43] R. D. McKeown. The Jefferson Lab 12 GeV Upgrade. J.Phys.Conf.Ser.312:032014,(2011). arXiv:1009.3503.

[44] The Science and Experimental Equipment for The $12 \mathrm{GeV}$ Upgrade of CEBAF. http://www.jlab.org/12GeV/CDR_for_NSAC_Town_Meeting_r3.pdf.

[45] http://hallaweb.jlab.org/physics/. 
[46] J. Alcorn et al. Basic Instrumentation for Hall A at Jefferson Lab.

Nucl.Instrum.Meth. A522 (2004) 294-346.

[47] M. Iodice et al. Nucl. Inst. and Meth. A411, 223 (1998).

[48] K. G. Fissum et al. Nucl. Inst. and Meth. A474, 108 (2001).

[49] Hall A Operations Manual.

hallaweb.jlab.org/document/OPMAN/.

[50] W. Barry, et al., Beam position measurement in the CEBAF recirculating linacs by use of pseudorandom pulse sequences. JLab-TN-90-246, 1990; Basic noise considerations for CEBAF beam position monitors, JLab-TN-91-087, (1991).

[51] K. Unser, IEEE Trans. Nucl. Sci. NS 28 (1981) 234.

[52] J. Berthot, P. Vernin, Nucl. Phys. News 9 (1990) 12.

[53] M. Baylac, et al., Phys. Lett. B 539 (2002) 8.

[54] M. Mazouz. PhD thesis, Université Joseph Fourier (2006).

[55] W.A. Watson, et al., CODA: a scalable, distributed data acquisition system, in: Proceedings of the Real Time (1993) Conference, p. 296; G. Heyes, et al., The CEBAF on-line data acquisition System, in: Proceedings of the CHEP Conference, (1994), p. 122; G. Heyes, et al., Real-time front end software for CODA, in: Proceedings of the real time (1993) Conference, p. 382; D.J. Abbott, et al., CODA Performance in the real world, 11th IEEE NPSS Real Time (1999) Conference, JLab-TN-99-12, (1999).

[56] E. Jastrzembski, et al., The Jefferson Lab trigger supervisor system, 11th IEEE NPSS Real Time 1999 Conference, JLab-TN-99-13, (1999).

[57] M. Mazouz, Calo timing optimization, Internal note (2012). https://hallaweb.jlab.org/dvcslog/DVCS2/150.

[58] Alejandro Martí, Angular resolution and calo offset, Internal note (2012). https://hallaweb.jlab.org/dvcslog/DVCS2/137.

[59] Alejandro Martí, Resolution and coefficient comparison of the calorimeter, Internal note (2011). https://hallaweb.jlab.org/dvcslog/DVCS2/65.

[60] M.Crouau, PMT gain changes, Internal note (2012). https://hallaweb.jlab.org/dvcslog/DVCS2/139.

[61] P. Y. Bertin, C. Hyde-Wright, F. Sabatié, E. Voutier et al., Jefferson Lab proposal E00-110 (2000). 
[62] Maxime Defurne, Calculation of the Cerenkov efficiency, Internal note (2013). https://hallaweb.jlab.org/dvcslog/DVCS2/167.

[63] M. Mazouz, S1, S2 and VDC efficiencies, Internal note (2011). https://hallaweb.jlab.org/dvcslog/DVCS2/38.

[64] Rafayel Paremuzyan, Maxime Defurne, Implementation of the Hall A DVCS calorimeter in GEANT4, Internal note (2013). https://hallaweb.jlab.org/dvcslog/DVCS2/145.

[65] Marat Rvachev, Effective use of JLab Hall A HRS acceptance with R-functions (2011). http://hallaweb.jlab.org/publications/Technotes/files/2001/01-055.ps.

[66] Maxime Defurne, HRS acceptance and Rfunction, Internal note (2013). https://hallaweb.jlab.org/dvcslog/DVCS2/226.

[67] Murat M. Kaskulov, Ulrich Mosel, Exclusive pion electroproduction off nucleons and nuclei. http://arxiv.org/abs/1103.1602 (2011).

[68] Carlos Muñoz Camacho, PhD thesis. Université Paris VI (2005).

[69] Maxime Defurne, Deeply Virtual Compton Scattering cross sections from the E00-110 experiment, Internal note (2013). https://hallaweb.jlab.org/dvcslog/DVCS2/242.

[70] J. Roche et al., Phys. Rev. Lett. 85, 708 (2000).

[71] M. Vanderhaeghen, J.M. Friedrich, D. Lhuillier, D. Marchand, L. Van Hoorebeke and J. Van de Wiele, QED radiative corrections to virtual Compton scattering, Phys.Rev. C62, 025501 (2000).

[72] Y. S. Tsai, Rev. Mod. Phys. 815, 46 (1974).

[73] X. Jiang, PhD thesis, University of Massachusetts Amherst.

[74] Charles E. Hyde-Wright, Comment on Radiative Corrections in Virtual Compton Scattering. Internal note (2006). https://hallaweb.jlab.org/dvcslog/DVCS2/235.

[75] L.W. Mo, Y. S. Tsai, Rev. Mod. Phys. 41, 205 (1969).

[76] S. Agostinelli et al. GEANT 4-A simulation toolkit. Nuclear Instruments and Methods in Physics Research A 506 (2003) 250-303.

[77] M. Mazouz, A. Ben Fredj, L. Ghedira, Measurement of the Deeply Virtual Compton Scattering cross-section off the neutron, Proposal for Jefferson Lab, E08-025 (2007). 
[78] Carlos Muñoz Camacho et al., Scaling Tests of the Cross Section for Deeply Virtual Compton Scattering, Phys.Rev.Lett.97:262002 (2006).

[79] M. Mazouz et al., Deeply virtual compton scattering off the neutron, Phys.Rev.Lett.99:242501 (2007). arXiv:0709.0450v2.

[80] J. Roche, C. E. Hyde-Wright, B. Michel, C. Munoz Camacho, et al., Measurements of the electron-helicity dependent cross sections of Deeply Virtual Compton Scattering with CEBAF at 12 Gev, Jefferson Lab PAC-30 proposal, E12-06-114 (2006). arXiv:nucl-ex/0609015v1. 



\section{Resumen en español}

El estudio de la estructura interna de los hadrones nos permite comprender la naturaleza de las interacciones entre los partones, quarks y gluones, descritas por la Cromodinámica Cuántica. En este marco se incluyen los procesos de difusión elástica, que han sido utilizados satisfactoriamente para medir los factores de forma de los nucleones. En este marco también se incluyen los procesos inelásticos, de los cuales es posible extraer una gran cantidad de información, gracias al desarrollo teórico de las Distribuciones de Partones (PDFs). Por tanto, mientras que la dispersión elástica de electrones por el nucleón nos proporciona información sobre la distribución de carga del mismo y, por ende, de la distribución espacial de sus componentes, la dispersión inelástica nos proporciona información sobre la distribución de momentos a través de las PDFs. No obstante, dentro de los procesos inelásticos, es posible estudiar procesos exclusivos tales como el Deeply Virtual Compton Scatering (DVCS), que nos permite el acceso a la distribución espacial y a la distribución de momentos de los quarks simultáneamente. Esto es posible a través de las denominadas Funciones Generalizadas de Partones (GPDs), que permiten correlacionar ambos tipos de distribuciones. El proceso conocido como DVCS es la forma más sencilla de acceder a las GPDs. Este proceso consiste en la dispersión de un electrón por un protón, mediante el intercambio de un fotón virtual, con el resultado de la dispersión de las partículas iniciales y la emisión de un fotón real.

$$
p+e \rightarrow p^{\prime}+e^{\prime}+\gamma
$$

Este proceso compite con el denominado Bethe-Heitler, en el que el fotón real es emitido por el electrón inicial o final. Debido a la pequeña sección eficaz de este tipo de procesos, del orden del $n b$, es necesario hacer uso de unas instalaciones capaces de proporcionar una alta luminosidad para llevar a cabo los experimentos. Una de estas instalaciones es el Thomas Jefferson National Accelerator Facility, donde se condujo el experimento denominado "Complete Separation of Virtual Photon and $\pi^{0}$ Electroproduction Observables of Unpolarized Protons", durante el periodo entre octubre y diciembre de 2010. El principal objetivo de este experimento es la separación de la contribución proveniente del DVCS a partir del término de interferencia, resultante de la contribución del BH. Esta separación 
recibe el nombre de "Separación Rosenbluth".

El Jefferson Laboratory se encuentra situado en la ciudad de Newport News, Virginia (U.S.A.). Su principal instalación es el acelerador CEBAF (Continous Electron Beam Accelerator Facility). Está diseñado para que los electrones alcancen hasta $6 \mathrm{GeV}$, trazando hasta 5 órbitas a lo largo de sus dos Linacs superconductores, de unos $300 \mathrm{~m}$ de longitud, conectados por secciones curvas en los extremos. Está equipado con un inyector capaz de proporcionar electrones polarizados. El haz es canalizado hasta 3 instalaciones distintas conocidas como Halls $A, B$ y $C$. Cada una de estas instalaciones puede recibir simultáneamente un haz con energía máxima, y dispone del equipo de detección necesario para almacenar los resultados de la colisión del haz con diferentes blancos estacionarios. La construcción de un nuevo Hall (D) se está llevando a cabo en el extremo opuesto al emplazamiento del resto de los Halls. Actualmente, se está trabajando en las mejoras para incrementar la energía del haz hasta los $12 \mathrm{GeV}$ y se espera que el acelerador esté operativo a $12 \mathrm{GeV}$ a lo largo del 2014.

El Hall A, donde se llevó a cabo la experiencia, está destinado a experimentos de alta precisión con una alta luminosidad. Entre su equipamiento destacan dos espectrómetros de alta resolución. A lo largo de la trayectoría del haz hay dispuestos varios sistemas de medida con el objetivo de recoger información sobre el mismo. Los polarímetros, Compton y Møller, determinan la polarización del haz. El primero permite la medición sin necesidad de detener el proceso experimental mientras que las mediciones del segundo son intrusivas. También se dispone de sistemas de monitorización de la energía y corriente de haz. Es posible conocer la posición del centro del haz con una indeterminación de $100 \mu m$ para corrientes de $1 \mu \mathrm{A}$.

El montaje experimental fue el siguiente: el haz de electrones impacta en un blanco de $\mathrm{LH}_{2}$ con el resultado de la dispersión de un protón, un electrón (detectado por uno de los espectrómetros) y la emisión de un fotón (detectado por un calorímetro electromagnético diseñado para la experiencia). Esta tesis se centra en el análisis de los datos recogidos por el calorímetro, para la obtención de la sección eficaz de la reacción descrita, y la realización de la Separación Rosenbluth de la contribución del DVCS al término de interferencia. El calorímetro está conformado por 208 bloques de $P b F_{2}$, cada uno con dimensiones $3 \times 3 \times 18.6 \mathrm{~cm}^{3}$. Debido a sus características, más del $99.9 \%$ de la energía incidente es absorbida por el cristal. Los fotones Čerenkov emitidos por las partículas cargadas que conforman las cascadas electromagnéticas son recolectados por fotomultiplicadores.

En primer lugar se abordó la calibración del calorímetro. El calibrado consiste en la obtención de los coeficientes que nos permitan obtener la energía depositada en función de la amplitud del pulso electrónico de cada bloque. Para ello, se estudió la respuesta del 
calorímetro a los impactos de electrones provenientes de la reacción elástica $p+e \rightarrow p^{\prime}+e^{\prime}$. En este caso, el protón dispersado era detectado por el espectrómetro. Dado que se trata de una reacción elástica, es posible conocer las variables cinemáticas del electrón dispersado en función de las variables correspondientes al haz de electrones, el blanco y el protón dispersado. Conociendo la energía de los electrones que son detectados por el calorímetro, es posible convertir la amplitud de las señales generadas por estos en unidades de energía. Al mismo tiempo estudiamos la resolución energética y angular del aparato. Todo ello teniendo en cuenta que típicamente la cascada electromagnética se produce en nueve bloques, el impactado y sus adyacentes. Es por eso que cuando tenemos un impacto en uno de los bordes del calorímetro no registamos parte de la energía de la partícula. Esto influye en gran modo en la calibración del detector: los coeficientes de calibración de los bloques situados en los extremos no son tan fiables como el resto. La calibración puede variar con el paso del tiempo, es por ello que se llevaron a cabo varias sesiones durante las fechas en las que duró el experimento. La estabilidad obtenida para los coeficientes a lo largo de las diferentes calibraciones nos indica la fiabilidad de los mismos. Los resultados obtenidos para las resoluciones energética $(\sim 3 \%)$ y angular son también satisfactorios.

Tras la calibración del detector comenzó el análisis de las señales procedentes del experimento en sí. Debido a la alta luminosidad del haz de electrones es necesario lidiar con mucho ruido de fondo y eventos con "pile-up". Cuando hay una detección, las señales generadas por cada bloque son almacenadas durante 128 ns. Al observar las señales apreciamos la variación en la corriente generada por los fotomultiplicadores como respuesta a la energía depositada por las particulas: un pulso cuando hay un solo impacto, dos pulsos (pile-up) o ruido de fondo. Por lo tanto, fue necesario implementar un algoritmo que extrajera la amplitud y el tiempo de llegada de los pulsos de forma acorde al tipo de evento registrado. Al mismo tiempo, se introdujeron las correcciones al tiempo de llegada debidas a las diferencias en la longitud de los cables y la posición de los bloques que conforman el calorímetro.

A la hora de reconstruir la energía del electrón dispersado no tiene sentido utilizar las señales de todos los bloques del calorímetro, pues es obvio que $e^{\prime}$ no deposita energía en todos ellos. Si lo hicieramos estaríamos añadiendo mucha contribución proveniente del ruido electrónico. Es pues muy conveniente, determinar cuáles son los bloques en los que $e^{\prime}$ ha depositado energía. Un modo de determinar qué bloques han recogido energía del $e^{\prime}$ es imponer un valor umbral en la amplitud de la señal. No obstante, un valor umbral para la amplitud de cada bloque no es el prodecimiento adecuado, pues la mayoría de la energía del electrón (alrededor de un 90\%) se deposita en un único bloque, mientras que el resto se distribuye por los bloques colindantes. La forma en que abordamos la reconstrucción de la energía es imponiendo un valor umbral a la suma de las amplitudes de cada combinación posible de grupos de 4 bloques adyacentes. Una vez seleccionados los grupos de cuatro que superan el umbral, un algoritmo conocido como "clustering", 
creado para formar "clusters" (combinaciones de bloques que determinan la zona de impacto de una partícula), busca el máximo local y forma las zonas de impacto añadiendo bloques colindantes con una amplitud menor a los bloques ya añadidos. De este modo, es posible extraer la información necesaria para el cálculo de las variables cinemáticas de las partículas que impactan en el detector, que empleamos en el cálculo de la sección eficaz.

Durante la adquisición de datos tenemos contribuciones de reacciones que no pertenecen al DVCS. Estas detecciones alteran nuestros datos y deben ser sustraídas. La mayoría de estas detecciones son descartadas aplicando algunos cortes en determinadas variables cinemáticas, como por ejemplo, en el punto de impacto en el blanco. Debido al encapsulado del blanco no todos los protones dispersados provienen del $\mathrm{LH}_{2}$. Podemos reconstruir el punto de impacto del electrón incidente con los datos del HRS para comprobar si hubo colisión con el blanco. En el caso del HRS, se aplica un corte a las variables cinemáticas del electrón dispersado con el fin de seleccionar eventos dentro de la región de aceptancia óptima del espectrómetro. También se aplican cortes a las zonas de impacto del calorímetro, descartando aquellas detecciones en las partes exteriores del aparato. Esto es debido a la pérdida de energía en estas zonas.

No toda la extracción de detecciones no deseadas puede ser llevada a cabo empleando cortes en variables cinemáticas. La sustracción de la contribución proveniente de la electroproducción de $\pi^{0}$ debe ser realizada empleando otros métodos. Un método sería el de evaluar la sección eficaz de esta reacción dentro del ángulo sólido del experimento. Sin embargo, hemos optado por emplear una simulación en la que generamos desintegraciones de $\pi^{0}$, con la cual el cálculo anterior es innecesario. Este método genera desintegraciones de $\pi^{0}$ en dos fotones, con el fin de evaluar la cantidad de impactos sobre el calorímetro por parte de uno de los fotones que provienen de esta reacción (es en este caso cuando se contaminan nuestros datos puesto que el DVCS sólo genera un fotón real). Asimismo, también es necesario evaluar la cantidad de impactos accidentales a la hora de recoger datos. Para ello se emplea el espectro de detecciones del calorímetro cuando el tiempo de llegada de las partículas no coincide con el del HRS. Puesto que el espectro de detecciones accidentales es continuo, debe ser equivalente en cualquier momento.

Tras realizar la sustracción del fondo debemos aplicar correcciones debidas a la radiación bremsstrahlung creada por los electrones al atravesar el blanco. Esta corrección se encuadra dentro de las correcciones radiativas externas, que aplicamos a los datos antes de calcular la sección eficaz. Las correcciones radiativas internas se dividen en correcciones virtuales y correcciones reales. Las correcciones virtuales incluyen correcciones de vértice y de polarización del vacío, mientras que las correcciones reales abordan la emisión de fotones reales por los electrones. Una vez aplicadas las correcciones radiativas comenzamos el cálculo de la sección eficaz. Para ello hacemos uso de una simulación Monte Carlo, con la finalidad de incluir la resolución y geometría de los aparatos empleados en el 
cálculo. En la simulación se introducen los aparatos principales que conforman el experimento: la cámara de dispersión (donde se encuentra el blanco), los tubos conductores del haz de electrones, el calorímetro y el HRS. La simulación requiere incluir los materiales que conforman los aparatos, con sus respectivas propiedades. También se introducen las propiedades del medio que rodea los aparatos.

El cómputo de la sección eficaz nos permite obtener las diferentes contribuciones provenientes del BH y de los "Compton Form Factors" $(\mathrm{CFFs}), \mathcal{C}_{\text {unp }}^{\mathcal{I}}(\mathcal{F}), \Delta \mathcal{C}_{\text {unp }}^{\mathcal{I}}(\mathcal{F})$ y $\mathcal{C}_{\text {unp }}^{\mathcal{I}}\left(\mathcal{F}^{\text {eff }}\right)$ (factores asociados a la descomposición en harmónicos de Fourier de la interferencia generada por el DVCS y el BH). Durante el experimento se estudiaron tres cinemáticas distintas, variando la virtualidad del fotón. Para cada una de estas cinemáticas se emplearon dos haces de energía diferentes, con el fin de aislar los factores cinemáticos, independientes de la energía del haz. Esto abre la posibilidad de realizar la Separación Rosenbluth de la contribución del DVCS al término de interferencia. Se ha realizado un "binning" en la variable $t$ ( $t$ corresponde a la transferencia de momento que recibe el protón, expresada al cuadrado), lo que nos permite obtener diez diferentes secciones eficaces para cada cinemática estudiada (cinco por cada bin en $t$ y dos por cada energía del haz), en función del ángulo entre los planos hadrónico y leptónico de la reacción, $\varphi_{\gamma}$. En esta tesis se presentan los resultados preliminares del análisis de los datos recogidos. En todos los casos observamos una desviación de la sección eficaz total de la contribución del BH. Para energías mayores, la desviación es mayor. También observamos que el comportamiento de las distintas contribuciones es muy similar al encontrado en experimentos anteriores.

Con los datos de los que disponemos ya es posible realizar la Separación Rosenbluth y así obtener la contribución del $\operatorname{CFF} \mathcal{C}_{\text {unp }}^{D V C S}\left(\mathcal{F}, \mathcal{F}^{*}\right)$, empleando los resultados mostrados en esta tesis. Sin embargo, esta tesis no incluye este cálculo, que queda para un futuro trabajo. También, quedan por incluir las contribuciones de los errores sistemáticos al cálculo de los errores en las secciones eficaces. Un estudio sobre el Deep Inelastic Scattering (DIS), realizado empleando los datos del experimento, indica que las contribuciones de los errores sistemáticos al DIS se sitúan en torno al 3.5\%. Partiendo de este dato se espera una contribución no superior al $5 \%$ para el caso del DVCS. En un futuro, queda como objetivo el cálculo de las secciones eficaces polarizadas. 



\section{Résumé en français}

Une particule élémentaire est une particule dont la structure est inconnue, il est donc impossible de savoir si elle est compoée d'autres particules. Historiquement, les particules constitutives de l'atome (électrons, protons et neutrons) ont toutes été considérées comme des particules élémentaires. Cependant, avec la découverte du moment magnétique du proton [1], il est devenu clair que le proton n'appartenait pas à cette catégorie. Avec d'autres expériences, plus de preuves ont constaté que les protons et les neutrons, ainsi que tous les hadrons, sont composés d'autres particules, et qu'ils ont une structure interne [2], [3]. La premiére preuve de quarks comme éléments constitutifs des hadrons a été obtenue à la fin de 1967, quand la première d'une longue série d'expériences sur la diffusion inélastique d'électrons eut lieu au Stanford Linear Accelerator Center (SLAC) [4]. Les taux de comptage étaient beaucoup plus élevés qu'ils avaient été prévu dans la région inélastique profonde, où l'électron donne la plupart de son énergie au proton. L'expérience a montré que la probabilité de la diffusion inélastique profonde diminue beaucoup plus lentement avec le transfert d'impulsion invariant au proton, que celle pour la diffusion élastique [5]. Une façon d'interpréter ce comportement inattendu est que les électrons ont frappé une sorte de noyau dur à l'intérieur des protons. La première preuve de l'existence des gluons est venu dans des événements à trois jets à PETRA [6].

Dans l'été de 1973 physiciens de Harvard et Princeton ont démontré que, dans certaines théories "gauge" la force entre les quarks pourrait devenir relativement faible sur de courtes distances, un comportement connu comme liberté asymptotique [7], ce qui fait des liens entre les particules devenir asymptotiquement plus faible quand l'énergie augmente et la distance diminue. Une autre propriété des interactions entre les quarks, appelée confinement, affirme que la force entre les quarks ne diminue pas quand ils sont séparés. Pour cette raison, lorsque deux quarks sont séparés, comme c'est le cas dans les collisions de particules dans l'accélérateur, à un moment donné, il est énergétiquement plus favorable pour une nouvelle paire quark-antiquark d'apparaître spontanément, que de laisser la distance s'étendre encore. Même si le confinement n'a pas été analytiquement prouvée, il est largement soupçonné d'être vraie parce qu'il explique l'échec des recherches 
de quarks libres. Il n'a pas de ligne de transition connue entre ces deux propriétés. Le confinement est dominant à faible énergie mais, avec l'augmentation de l'énergie, la liberté asymptotique devient dominante. Ces interactions entre partons, les quarks et les gluons, sont décrites par la chromodynamique quantique (QCD).

Aujourd'hui, la diffusion inélastique profonde (DIS) continue à offrir un chemin pour extraire des nouvelles informations sur la structure hadronique, et par conséquent, sur les inconnues de la façon dont QCD fonctionne. Cette thèse est basée sur l'expérience de Jefferson Lab E07-007 [8], qui cherche à exploiter ce genre de processus, et plus particulièrement, la diffusion Compton virtuel (VCS), qui est une réaction exclusive de DIS. Le VCS consiste en la production d'un photon réel, provenant de la dispersion un photon virtuel par le nucléon,

$$
\gamma^{*}+p \rightarrow \gamma+p^{\prime}
$$

Ce genre de réactions, dans un certain régime cinématique appelé Diffusion Compton Profondément Virtuelle (DVCS), peuvent fournir des informations intéressantes sur une nouvelle classe d'éléments de matrice, appelés Distributions de Partons Généralisées (GPDs). Les GPDs montrent la corrélation entre la distribution spatiale transversale du parton frappé (quark ou gluon) et la fraction d'impulsion dans le cône de lumière de ce parton. Cela nous fournit des informations sur la distribution spatiale et des impulsions des partons dans le nucléon, qui nous aide à comprendre comment le comportement des partons confère ces caractéristiques au nucléon.

En raison de la petite section efficace du DVCS (de l'ordre du $n b$ ), afin de réaliser ce genre d'expériences, il est nécessaire de faire usage d'installations capables de fournir un faisceau avec des intensités élevées. L'un de ces installations est le Thomas Jefferson National Accelerator [9], où l'expérience JLab E07-007 [10], "Complete Separation of Virtual Photon and $\pi^{0}$ Electroproduction Observables of Unpolarized Protons ", eut lieu pendant les mois d'Octobre à Décembre de 2010. J'ai commencé ma collaboration avec l'expérience plusieurs mois après l'acquisition des données.

- Le chapitre 1 est une introduction théorique à l'étude de la structure du nucléon, en révisant les concepts de facteurs de forme et des distributions de partons à travers des processus élastiques et inélastiques. Le calcul de la section efficace de la leptoproduction de photons est décrite en détail, ainsi que les buts de l'expérience E07-007.

- Le chapitre 2 est une description des principales caractéristiques du Jefferson Lab, en mettant l'accent sur le Hall A, où l'expérience a eu lieu, et son instrumentation. 
Le dispositif expérimental avec les cinématiques employées pendant l'acquisition des données peut être trouvé dans ce chapitre. Détail particulier est accordé au calorimètre électromagnétique, le dispositif sur lequel la plupart des travaux de cette thèse sont basés.

- Le chapitre 3 décrit l'analyse des données enregistrées par le calorimètre électromagnétique, avec le but d'obtenir les variables cinématiques des photons réels résultants des réactions DVCS. Ce chapitre comprend également le procédé de calibration de cet appareil ainsi que le calcul de son résolution en énergie et de son résolution angulaire.

- Le chapitre 4 décrit la sélection des événements à partir des données stockées, les réductions appliquées aux variables cinématiques et la soustraction de fond. En outre, le processus d'extraction des observables nécessaires pour le calcul de la section efficace de la leptoproduction de photons est décrite, ainsi que les principales étapes suivies pour effectuer la simulation Monte-Carlo utilisée dans ce calcul. Les sections efficaces obtenues sont indiquées à la fin de ce chapitre.

L'étude de la structure interne des hadrons nous permet de comprendre la nature des interactions entre les partons, les quarks et les gluons, décrites par la Chromodynamique Quantique. Les processus de diffusion élastique, qui ont été utilisés avec succès pour mesurer les facteurs de forme des nucléons, sont inclus dans ce cadre. Les processus inélastiques sont également inclus dans ce cadre, ils nous permettent d'extraire beaucoup d'information grâce au développement des distributions de partons (PDFs). Par conséquent, tandis que la diffusion élastique d'électrons par le nucléon nous fournit des informations sur la répartition des charges, et donc de la distribution spatiale des composants du nucléon, la diffusion inélastique présente des informations sur la distribution d'impulsions au moyen des PDFs. Cependant, dans les processus inélastiques, il est possible d'étudier les processus exclusifs tels que la Diffusion Compton Profondément Virtuelle (DVCS), qui nous permet d'accéder aux distributions spatiale et d'impulsions des quarks simultanément. Ceci est possible grâce aux fonctions généralisées des distributions de partons (GPDS), qui nous permettent de corréler les deux types de distributions. Le processus connu sous le nom DVCS est le moyen le plus facile pour accéder aux GPDS. Ce procédé implique la diffusion d'un électron par un proton, au moyen de l'échange d'un photon virtuel, qui entraîne la diffusion des particules initiales et l'émission d'un photon réel.

$$
p+e \rightarrow p^{\prime}+e^{\prime}+\gamma
$$

Ce processus est en concurrence avec le processus dit Bethe-Heitler, dans lequel le photon réel est émis par l'électron initial ou final. En raison de la faible section efficace de ce 
type de procédé, de l'ordre du nb, il est nécessaire d'utiliser une installation capable de fournir une haute luminosité pour réaliser les expériences. L'une de ces installations est le Thomas Jefferson National Accelerator Facility, où l'expérience appelée "Complete Separation of Virtual Photon and $\pi^{0}$ Electroproduction Observables of Unpolarized Protons" a été réalisée au cours de la période entre Octobre et Décembre de 2010. Le principal objectif de cette expérience est la séparation de la contribution du terme provenant du DVCS à partir du terme d'interférence, résultant de la contribution du BH. Cette séparation est appelée "Séparation Rosenbluth"

Jefferson laboratoire est situé dans la ville de Newport News, Virginia (U.S.A.). Son installation principale est l'accélérateur CEBAF (Continous Electron Beam Accelerator Facility). Il est conçu de sorte que les électrons atteignent jusqu'à $6 \mathrm{GeV}$, en parcourant jusqu'à cinq orbites au long de deux accélérateurs linéaires superconducteurs, d'environ $300 \mathrm{~m}$ de long, reliés par des sections courbes aux extrémités. Il est équipé d'un injecteur capable de fournir des électrons polarisés. Le faisceau est acheminé jusqu'à 3 établissements différents connus comme halls $\mathrm{A}, \mathrm{B}$ et $\mathrm{C}$. Chacune de ces installations peut recevoir simultanément un faisceau d'énergie maximale, et elles ont l'équipement nécessaire pour la détection et l'enregistrement des résultats de la collision du faisceau avec des différentes cibles fixes. Un noveau hall (D) est en train d'être construit à l'extrémité opposée du site. Actuellement, le laboratoire travaille sur des améliorations pour augmenter l'énergie de faisceau jusqu'à $12 \mathrm{GeV}$.

Le Hall $\mathrm{A}$, où l'expérience a eu lieu, est destiné à des expériences de haute précision à haute luminosité. Il est équipé avec deux spectromètres de haute résolution. Sur le long de la trajectoire du faisceau, plusieurs systèmes de mesure sont disposés afin de recueillir des informations sur les electrons. Les polarimètres, Compton et Møller, déterminent la polarisation du faisceau. Le premier permet de mesurer sans arrêter le processus expérimental tandis que les mesures du second dispositif sont intrusives. Des systèmes de mesure de l'énergie et le courant du faisceau sont aussi disponibles. Il est possible de connaitre la position du centre du faisceau avec une uncertitude de $100 \mu m$ pour des courants de $1 \mu \mathrm{A}$.

Le dispositif expérimental était le suivant: le faisceau d'électrons frappe une cible de $\mathrm{LH}_{2}$, résultant en la diffusion d'un proton, un électron (détecté par l'un des spectromètres) et l'émission d'un photon (détecté par un calorimètre électromagnétique conçu pour l'expérience). Cette thèse se centre sur l'analyse des données recueillies par le calorimètre, pour obtenir la section efficace de la réaction décrite et pour réaliser la Séparation Rosenbluth de la contribution du terme DVCS au terme d'interférence. Il est composé de 208 blocs de $\mathrm{PbF}_{2}$, chacun mesurant $3 \times 3 \times 18.6 \mathrm{~cm}^{3}$. Grâce à ses caractéristiques, plus de 99,9\% de l'énergie incidente est absorbée par le verre. Les photons Čerenkov émis par les particules chargées qui composent les cascades électromagntiques sont recueillies par des photomultiplicateurs. Autour de 1000 photons Čerenkov sont émis par GeV (estimation 
par simulation Monte Carlo).

Tout d'abord la calibration du calorimètre a été adressée. La calibration consiste à obtenir des coefficients qui nous permettent d'obtenir l'énergie déposée en fonction de l'amplitude de l'impulsion électronique de chaque bloc. À cet effet, la réponse du calorimètre à l'impact d'électrons provenants de la réaction élastique $p+e \rightarrow p^{\prime}+e^{\prime}$ a été étudiée. Dans ce cas, le proton diffusé a été détecté par le spectromètre. Comme il s'agit d'une réaction élastique, il est possible de connaître les variables cinématiques de l'électron diffusé en fonction des variables correspondantes au faisceau d'électrons, la cible et le proton diffusé. Connaissant l'énergie des électrons qui sont détectés par le calorimètre, il est possible de convertir l'amplitude des signaux générés en unités d'énergie. Au même temps nous étudions la résolution énergétique et angulaire de l'appareil. Tout cela en gardant à l'esprit que généralement la cascade électromagnétique se produit en neuf blocs, l'impacté et ses adjacentes. C'est pourquoi, lorsque nous avons un impact sur l'un des bords du calorimètre, une partie de l'énergie de la particule n'est pas détecté. Cela influe fortement sur la calibration du détecteur: les coefficients des blocs aux extrémités ne seront pas aussi fiables que les autres. La calibration peut varier au fil du temps, c'est pourquoi nous avons fait plusieurs séances durant l'expérience. La stabilité obtenue pour les coefficients pendant les différentes séances indique la fiabilité de ceux-ci. Les résultats obtenus pour les résolutions angulaires et énergétique sont également satisfaisants.

Après la calibration du détecteur j'ai commencé l'analyse des signaux de l'expérience. En raison de la forte luminosité du faisceau d'électrons, il est nécessaire de traiter beaucoup de bruit de fond et événements avec "pile-up". Lorsqu'il y a une détection, des signaux générés par chaque bloc sont stockés pendant 128 ns. En observant les signaux nous pouvons apprécier la variation du courant généré par la réaction des photo-multiplicateurs à l'énergie déposée par les particules: une impulsion quand il y a un seul impact, deux impulsions (pile-up) ou le bruit de fond. Par conséquent, il était nécessaire de mettre en oeuvre un algorithme pour établir l'amplitude et le temps d'arrivée des impulsions en fonction du type d'événement enregistré. Au même temps, des corrections au temps d'arrivée ont été introduites. Ces corrections sont nécessaires à cause des différences dans la longueur des câbles et la position des blocs du calorimètre.

Pendant la reconstruction de l'énergie de l'électron diffusé il n'a pas de sens d'utiliser les signaux de tous les blocs dans le calorimètre, alors qu'il est évident que l' $e^{\prime}$ ne dépose pas de l'énergie dans chacun d'eux. Si nous le faisions, nous ajouterions beaucoup du bruit électronique. Il est donc hautement souhaitable, de déterminer quels sont les blocs dans lesquels l'e déposé son énergie. Une façon de déterminer quels blocs ont collecté de l'énergie de l'e $e^{\prime}$, c'est d'imposer un seuil sur l'amplitude du signal. Toutefois, une valeur du seuil pour l'amplitude de chaque bloc n'est pas la procédure appropriée, parce que la plupart de l'énergie de l'électron (environ 90\%) est déposée en un seul bloc, tandis que le 
reste est distribué parmi les blocs adjacents. La façon dont nous abordons la reconstruction de l'énergie, c'est d'imposer une valeur de seuil à la somme des amplitudes de chaque combinaison possible de groupes de 4 blocs adjacents. Une fois sélectionnés les combinaisons de groupes de 4 blocs adjacents qui dépassent le seuil, un algorithme appelé "clustering" créé pour former des "clusters" (combinaisons de blocs qui déterminent l'impact d'une particule), recherche le maximum local et creé les zones d'impact en ajoutant des blocs adjacents avec une amplitude en dessous de celle des blocs adjacents dejà ajoutés. Ainsi, il est possible d'extraire les informations nécessaires pour le calcul des paramètres cinématiques des particules qui impactent le détecteur, que nous allons utiliser dans le calcul de la section efficace.

Pendant l'acquisition de données nous trouvons des contributions provenants de réactions qui n'appartiennent pas au DVCS. Ces détections modifient nos données et doivent être soustraites. La plupart de ces détections sont rejetées par l'application de certaines coupures sur certaines variables cinématiques, par exemple, le point d'impact sur la cible. À cause des parois de la cible, les protons diffusés ne proviennent pas tous du $\mathrm{LH}_{2}$. Nous pouvons reconstruire le point d'impact des électrons incidents avec les données obtenues du HRS, et voir s'il y avait collision avec la cible. Dans le cas du HRS, une coupure sur les variables cinématiques de l'électron diffusé afin de sélectionner les événements dans la région optimale de l'acceptance est appliquée. Des coupures dans les zones d'impact du calorimètre sont également appliqués, en écartant les détections dans les parties extérieures de l'appareil. Cela est dû à la perte d'énergie dans ces zones.

La rejection de toutes les détections indésirables ne peut pas être effectuée uniquement en utilisant des coupures sur les variables cinématiques. La soustraction de la contribution de la electroproduccion de $\pi^{0}$ doit être faite en utilisant d'autres méthodes. Une méthode consisterait à évaluer la section efficace de cette réaction dans l'angle solide de l'expérience. Cependant, nous avons choisi d'utiliser une simulation dans laquelle nous générons des désintégrations $\pi^{0}$, dont le calcul ci-dessus n'est pas nécessaire. Cette méthode génère des désintégrations $\pi^{0}$ en deux photons, afin d'évaluer le nombre d'impacts par un seul photon sur le calorimètre (c'est dans ce cas que les données sont contaminées, parce que le DVCS génère un seul photon réel). Il est aussi nécessaire d'évaluer la quantité de détections fortuites pendant la prise des données. Pour cette évaluation, nous avons utilisé le spectre de détections du calorimètre avec un temps d'arrivée des particules qui ne correspond pas à celui du HRS. Comme le spectre de détections fortuites est continu, il doit être équivalent à tout moment.

Après avoir effectué la soustraction du fond, nous devons appliquer des corrections dues au bremsstrahlung créé par les électrons en traversant la cible. Cette correction se situe dans les corrections radiatives externes appliquées aux données avant le calcul de la section efficace. Les corrections radiatives internes sont divisées en corrections virtuelles 
et corrections réelles. Les corrections virtuelles incluent les corrections de vertex et la polarisation du vide, tandis que les corrections réelles portent sur l'émission de photons réels par des électrons. Une fois appliquées les corrections radiatives on commence le calcul de la section efficace. Pour cela on utilise une simulation Monte Carlo, en vue d'inclure la résolution et la géométrie des appareils utilisés dans le calcul. Dans la simulation, des principaux appareils qui sont utilisés dans l'expérience, sont introduits: la chambre de diffusion (où la cible se trouve), les tubes du faisceau d'électrons, le calorimètre et le HRS. La simulation comprend les matériaux des appareils, avec leurs propriétés respectives. Les propriétés du milieu qui entoure les dispositifs sont également introduites.

Le calcul de la section efficace nous permet d'obtenir les différentes contributions provenantes du BH et des "Compton Form Factors", $\mathcal{C}_{\text {unp }}^{\mathcal{I}}(\mathcal{F}), \Delta \mathcal{C}_{\text {unp }}^{\mathcal{I}}(\mathcal{F})$ et $\mathcal{C}_{\text {unp }}^{\mathcal{I}}\left(\mathcal{F}^{\text {eff }}\right)$ (facteurs associés à la décomposition en harmoniques de Fourier de l'interférence générée par le DVCS et le BH). Pendant l'expérience, trois cinématiques ont été étudiées en faisant varier la virtualité du photon. Pour chacune de ces cinématiques deux faisceaux d'énergie différente ont été utilisés afin d'isoler des facteurs cinématiques indépendantes de l'énergie du faisceau. Cela ouvre la possibilité de réaliser la Séparation Rosenbluth de la contribution du DVCS du terme d'interférence. On a fait un "binning" dans la variable $t$ ( $t$ correspond au carré de la quantité d'impulsion reçue par le proton), qui nous permet d'obtenir dix sections efficaces différentes pour chaque cinématique (cinq pour chaque bin en $t$ et deux pour chaque énergie de faisceau), en fonction de l'angle entre les plans hadronique et leptonique, $\varphi_{\gamma}$. Les résultats obtenus au cours de la réalisation de cette thèse sont préliminaires. Dans tous les cas, nous avons observé une déviation de la section efficace totale par rapport au BH. Pour des énergies plus élevées, la déviation est plus grande. Nous avons observé aussi que le comportement des différentes contributions sont très semblables à ceux trouvés dans les expériences précédentes.

Avec les données à notre disposition, il est possible d'effectuer la Séparation Rosenbluth, et d'obtenir la contribution du $\operatorname{CFF} \mathcal{C}_{\text {unp }}^{D V C S}\left(\mathcal{F}, \mathcal{F}^{*}\right)$, en utilisant les résultats présents dans cette thèse. Toutefois, cette thèse ne comprend pas ce calcul, qui reste comme travaux futurs. Il reste aussi à inclure les contributions des erreurs systématiques dans le calcul des erreurs des sections efficaces. Une étude sur la diffusion inélastique profonde (DIS), faite à partir des données de cette expérience, indique que les contributions des erreurs systématiques pour le DIS sont situées autour de 3,5\%. À partir de cette information, une contribution qui ne dépasse pas le 5\% dans le cas du DVCS est attendue. Dans l'avenir, il reste comme objectif le calcul des sections efficaces polarisées.

\section{Conclusions}

Cette thèse porte sur le calorimètre électromagnétique qui a été utilisé pour détecter le photon dans l'expérience E07-007 à Jefferson Lab. Bien que j'ai commencé mon doc- 
torat après l'acquisition de données, j'ai eu la chance de visiter Jefferson Lab pendant que l'expérience était en marche, et observer les performances du calorimètre. L'expérience E07-007 est encadré dans la deuxième génération d'expériences DVCS à Jefferson Lab. Cette expérience, avec l'expérience E08-025 [77] s'appuie sur le succès de la première génération d'expériences DVCS [78],[79]. Il y a une troisième génération d'expériences DVCS [80] à venir qui aura lieu une fois que l'améloriation de l'installation CEBAF est opérationnel. La prochaine expérience DVCS cherchera à obtenir des sections efficaces du DVCS à un plus large $\mathcal{Q}^{2}$, en utilisant l'énergie augmenté du faisceau (jusqu'à $12 \mathrm{GeV}$ ). Cela permettra de déterminer avec quelle précision l'amplitude "handbag" domine (ou pas) sur des "higher-twist" amplitudes. Le nombre des expériences DVCS menées montre l'importance et la valeur de l'information que nous esperons à trouver sur la structure du nucléon, des distributions des quarks et ses impulsions dans le nucléon.

En ce qui concerne le cadre théorique de la réaction photon leptoproduction, j'ai réalisé le calcul des CFFs et la factorisation en harmoniques de Fourier de la section efficace, comme expliqué dans la section 1.4. C'était une façon de se faire une idée directe sur l'objectif central des expériences DVCS, et sur notre niveau de compréhension de la structure du proton en ce moment.

La première tâche que j'ai prise lors du démarrage de cette thèse, était la calibration du calorimètre. Il a été conçu à des fins d'expérimentation DVCS, et il est une version améliorée du même dispositif utilisé dans des expériences DVCS précédentes. L'amélioration a été effectuée dans le but d'employer le détecteur dans l'expérience E07007. Pour effectuer la calibration, j’ai analysé les données stockées des sessions de calibration, appliqué plusieurs coupures sur des variables cinématiques afin d'améliorer l'efficacité du dispositif, et traité des questions concernant les blocs défectueux énoncés dans la section 3.3.3. Le fait que plusieurs sessions de calibration, en utilisant les processus élastiques, ont eu lieu au cours de l'expérience nous permet d'avoir une calibration fiable. La stabilité des coefficients, ainsi que le test en utilisant les désintégrations $\pi^{0}$, confirment cette déclaration. À travers les données de la calibration élastique, nous avons calculé les valeurs de l'énergie et de la résolution angulaire du calorimètre, qui sont satisfaisants. A priori, les résolutions verticales et horizontales devrait être similaires. Cependant, nous constatons que la résolution verticale est un peu pire que l'horizontale. Une raison à cela pourrait être que la résolution verticale du HRS est pire que l'horizontale (6 mrad en $x$ et 2 mrad en $y$ ).

Après avoir effectué la calibration, nous avons été en mesure d'analyser les données stockées pour chaque bloc du calorimètre. La première étape a consisté à rejeter "runs" avec des problèmes connus, qui les rendaient inutilisables pour l'analyse. L'échantillonnage du courant des PMTs en réponse des particules est une chose particulière dans le système d'acquisition de données. On pourrait avoir décidé de mettre un seuil sur l'énergie, afin de sélectionner les événements en fonction de la valeur maximale du signal. Toutefois, en 
raison de la forte luminosité du faisceau dans le Hall A, il est important d'être en mesure d'analyser les événements qui auraient eu rejetés en raison de pile-up si nous avions décidé de stocker les données de cette façon. Dans le cas de la cinématique Kin 3-high, nous avons trouvé environ $8 \%$ de blocs avec deux impulsions. À ce stade, j'ai implémenté l'algorithme d'analyse pour deux impulsions, et optimisé les différents paramètres montrés dans le Tableau 3.2. Afin d'augmenter la précision et la rapidité du processus d'analyse des signaux, j'ai realisé l'étude des différents seuils de l'analyse. J'ai aussi appliqué la correction du temps de chaque bloc sur chaque série de données stockées, en étudiant le temps d'arrivée des signaux stockées, comme expliqué dans la section 3.1.5.

L'algorithme de clustering est un outil puissant pour le calcul des points d'impact dans la surface du calorimètre, ainsi que pour discerner les différentes particules provenants de multiples impacts. Mon travail impliquant l'algorithme de clustering comprend sa modification afin de stocker les données provenants d'événements avec deux-cluster, ainsi que l'étude du seuil du clustering. Ce seuil nous permet de discriminer les événements en utilisant le nombre de clusters formés, mais une correction multicluster est nécessaire. En plus de fournir un moyen d'obtenir les variables cinématiques des particules qui frappent le calorimètre, essentiels pour le calcul de la section efficace, le clustering nous a permis d'effectuer la soustraction $\pi^{0}$ sans avoir eu à calculer la section efficace de ce type de contamination.

L'étape suivante dans le calcul des sections efficaces a été de réaliser la soustraction du fond à partir des données. Ici j'ai effectué la soustraction $\pi^{0}$ et la soustraction des détections fortuites. Pour la soustraction $\pi^{0}$, j'ai eu à mettre en oeuvre un algorithme qui simule la surface du calorimtre électromagnétique. Ensuite, j'ai effectueé la randomisation des désintégrations $\pi^{0}$, en tenant compte de la cinématique et la géométrie de chaque désintégration, aussi comme la réalisation d'une correction pour le point d'impact sur la cible et un calcul qui tient compte du fait que la plupart de l'énergie des particules est déposée à $7 \mathrm{~cm}$ de profondeur à l'intérieur du calorimètre, dans la direction de la ligne de vol de la particule. Pour le cas de la soustraction des détections fortuites, j'ai extrait l'échantillon de détections fortuites pour chaque run et puis, extrait chaque événement fortuit du bin correspondant aux événements DVCS, selon sa cinématique. Comme indiqué précédemment, une correction multicluster était nécessaire pour prendre en compte les détections DVCS trouvés dans les événements avec deux clusters. La correction inclus l'analyse de tous les événements avec deux clusters, suivant les mêmes étapes utilisées dans l'analyse des événements d'un cluster.

Les sections efficaces ont été obtenues en utilisant une simulation Monte Carlo de l'expérience. La simulation a été utilisé pour ajuster les données et obtenir les différentes contributions aux sections efficaces. J'ai calculé les sections en suivant les étapes indiquées dans la section 4.8. Toutes les coupures sur les variables cinématiques ont été 
appliquées aux données de la simulation avant d'effectuer l'extraction des observables. Ensuite, j'ai introduit les coefficients montrés dans l'annexe $\mathrm{C}$ dans le calcul, et realisé le "binning" des sections efficaces sur les variables $t$ et $\varphi_{\gamma}$. Après été passé par toutes ces tâches, j'ai finalement obtenu la section préliminaire de la leptoproduction de photons pour chaque cinématique utilisée dans l'expérience, ainsi que les contributions du BH, $\mathcal{C}_{\text {unp }}^{\mathcal{I}}(\mathcal{F}), \Delta \mathcal{C}_{\text {unp }}^{\mathcal{I}}(\mathcal{F})$, et $\mathcal{C}_{\text {unp }}^{\mathcal{I}}\left(\mathcal{F}^{\text {eff }}\right)$, comme le montrent les Figures 4.20-4.25.

Au cours des dernières semaines de ma thèse, j'ai commencé à réaliser la séparation Rosenbluth afin d'obtenir la contribution du terme $\mathcal{C}_{u n p}^{D V C S}\left(\mathcal{F}, \mathcal{F}^{*}\right)$. Cependant, le calcul de ce terme n'est pas pleinement mis en oeuvre et il reste aussi du travail à faire. De même, les contributions des différentes erreurs systématiques n'ont pas encore été inclus dans le calcul des erreurs de sections efficaces. Une autre étude de l'erreur systématique créée par la coupure dans la "Masse manquante" doit être effectué. Cela inclura l'étude de l'erreur dans les différentes cinématiques (hormis kin3-high), et l'application d'une coupure dans les différentes zones du calorimtre, en fonction de la résolution dans ces zones. Enfin, nous nous attendons à obtenir des sections efficaces polarisées, en utilisant les données disponibles sur la polarisation du faisceau. 



